\title{
Highly functionalized 2-oxopiperazine-based peptidomimetics: An approach to PAR1 antagonists
}

Ángel M. Valdivielso, ${ }^{a}$ Pilar Ventosa-Andrés, ${ }^{a}$ Francisco Tato, ${ }^{a}$ M. Ángeles Fernández-Ibañez, ${ }^{a}$ Ioannis Pappos, ${ }^{b}$ Nikos E. Tsopanoglou ${ }^{b}$ M. Teresa GarcíaLópez, ${ }^{a}$ Marta Gutiérrez-Rodríguez, ${ }^{a}$ Rosario Herranz ${ }^{a_{*}}$

${ }^{a}$ Instituto de Química Médica (CSIC), Juan de la Cierva 3, 28006 Madrid, Spain.

${ }^{b}$ Department of Pharmacology, Medical School, University of Patras, 26500 Rio-Patras, Greece.

\begin{abstract}
A series of pseudodipeptide-based chiral 1,3,4,5-tetrasubstituted-2-oxopiperazines has been designed and synthesized as potential PAR1 antagonists. These highly functionalized piperazines were synthesized from aromatic and basic amino acid derived $\Psi[\mathrm{CH}(\mathrm{CN}) \mathrm{NH}]$ pseudodipeptides through a four step pathway that involves reduction of the cyano group to build the 2-oxopiperazine ring, followed by selective functionalization at the $\mathrm{N}_{4^{-}}, \quad \mathrm{N}_{1}$-positions, and at the exocyclic moiety at position $\mathrm{C}_{5}$. This regioselective functionalization required the fine tuning of reaction conditions. All new compounds were screened as inhibitors of human platelet aggregation induced by the PAR1 agonist SFLLRN and as cytotoxic agents in human cancer cell lines. Some of the compounds displayed moderate PAR1 antagonist activity, while, others were cytotoxic at $\mu \mathrm{M}$ concentration. No correlation was observed between both types of activities.
\end{abstract}

Keywords: PAR1 antagonists; Peptidomimetics; 2-Oxopiperazines; $\alpha$-Amino nitriles; Platelet antiaggregant activity; Cytotoxicity.

\section{Introduction}

Thrombin induces multiple effects on a variety of cells, such as: platelets [1-4], endothelial and smooth muscle cells $[1,4,5]$, neurons and astrocytes in the nervous system [1, 4-10], immune and inflammatory cells [4, 11, 12], and tumor cells [13-18]. These effects are mediated by the activation of the protease-activated receptor 1 (PAR1) [19]. This activation requires the thrombin-catalyzed cleavage of the N-terminal extracellular domain of PAR1 at the $\mathrm{Arg}^{41} / \mathrm{Ser}^{42}$ peptide bond, which unveils the recognition sequence SFLLRN that acts as a tethered activation ligand.

"Corresponding autor: Fax: +34 915644853; Tel: +34 912587537; E-mail: rosario@iqm.csic.es 
As PAR1 is mainly expressed in platelets, where its activation induces aggregation, it has been proposed that PAR1 antagonists could be good antithrombotic agents without the hemorrhagic drawbacks of thrombin inhibitors. Based on this suggestion, PAR1 antagonists have been searched almost exclusively in relation to the cardiovascular system up to now [2, $3,20]$. However, numerous studies have shown that PAR1 is overexpressed in invasive and metastatic tumors and that its expression levels directly correlate with the degree of invasiveness of the cancer [17, 21-28]. Based on these facts, this receptor is starting to be also considered a promising target for cancer therapy [19].

The first potent PAR1 antagonists were SFLLRN-based peptidomimetic ureas, represented by the optimized antagonist RWJ-58259 (Figure 1) [29], where an indazole ring is used as scaffold to assemble three key pharmacophoric groups (an aromatic and two amines). Later, several laboratories have reported a few series of antagonists obtained from HTS of diverse libraries of non-peptide small molecules, followed by optimization [20, 30], such SCH530348 (named vorapaxar, Figure 1) [31], currently in Phase III clinical trials [32-34].

Mutagenesis, X-ray, and NMR studies have shown that the first thrombin/PAR1 interaction is produced between the exosite I of thrombin and the hirudin-like sequence of PAR1 $\left(\mathrm{K}^{51} \mathrm{YEPF}^{55}\right)$, and that this first interaction is essential and determinant for high thrombin/PAR1 affinity [35-46]. These studies have also indicated that the hydrophobic residues F34, I82, L65 and Y76, and the basic residues R67 and R73, of the exosite I of thrombin are important for high affinity. This knowledge prompted us to initiate a project directed to the search of new PAR1 antagonists based on the hot spots of the exosite I of thrombin for PAR1. As these hot spots are discontinuous and are not localized in a defined secondary structure, we decided to use a diversity oriented synthesis (DOS) strategy for the search of peptidomimetics. To this aim, we planned the synthesis of diverse small directed libraries of different scaffolds able to assemble, at least, one or two aromatic groups and one or two basic groups at variable distances and orientations. As a first result of this project, we have recently reported a library of ureas and thioureas of general formula A. Some of these derivatives showed moderate antiaggregant activity in a screen of inhibitors of human platelet aggregation induced by the PAR1 agonist SFLLRN [47]. 

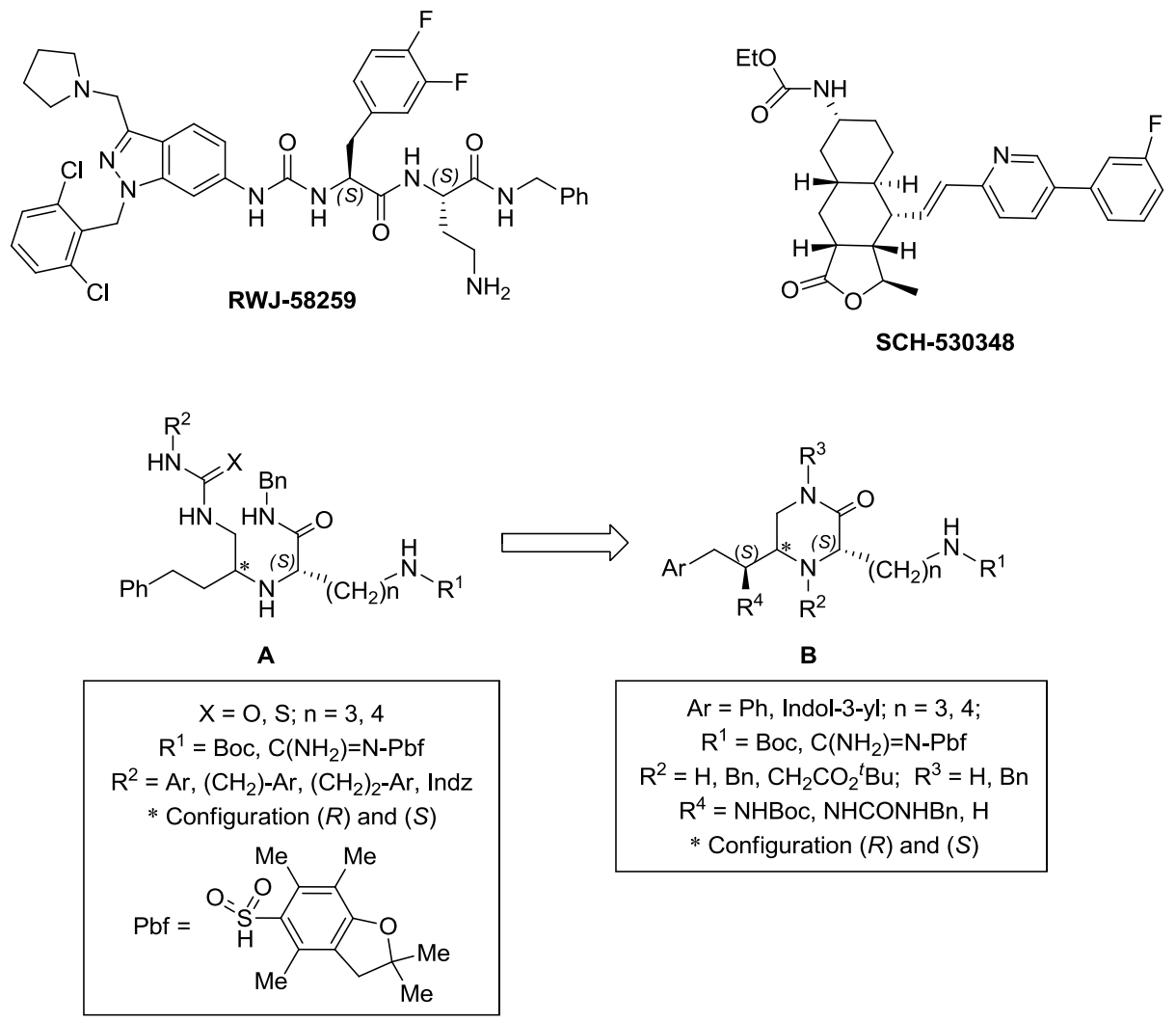

B
$\mathrm{Ar}=\mathrm{Ph}$, Indol-3-yl; $\mathrm{n}=3,4$; $\mathrm{R}^{1}=\mathrm{Boc}, \mathrm{C}\left(\mathrm{NH}_{2}\right)=\mathrm{N}-\mathrm{Pbf}$ $\mathrm{R}^{2}=\mathrm{H}, \mathrm{Bn}, \mathrm{CH}_{2} \mathrm{CO}_{2}{ }^{t} \mathrm{Bu} ; \mathrm{R}^{3}=\mathrm{H}, \mathrm{Bn}$ $\mathrm{R}^{4}=\mathrm{NHBoc}, \mathrm{NHCONHBn}, \mathrm{H}$ * Configuration $(R)$ and $(S)$

Fig. 1. Selected PAR1 antagonists and proposed 2-oxopiperazine derivatives B.

We now describe the synthesis and biological evaluation of a library of pseudodipeptidebased 2-oxopiperazine derivatives of general formula $\mathbf{B}$, which could be considered as conformational restricted analogues of the urea derivatives $\mathbf{A}$. The 2-oxopiperazine skeleton was selected as central core taking into account our synthetic experience in this heterocycle $[48,49]$ and that the piperazine ring is included among privileged scaffolds in medicinal chemistry. Actually, there are 165 drug entries for this heterocycle in the DrugBank database [50]. As in the series of urea derivatives $\mathbf{A}$ the side chain protection of the basic amino acid was necessary for their biological activity, the 2-oxopiperazine derivatives $\mathbf{B}$ were designed maintaining this protection. These piperazine derivatives have been screened as human PAR1 antagonists in a platelet aggregation assay and as cytotoxic agents in human cancer cell lines.

\section{Results and discussion}

\subsection{Synthesis}


As shown in the retrosynthetic Scheme 1, a molecular diversity generation strategy in four steps was devised for the synthesis of the 2-oxopiperazine derivatives $\mathbf{B}$, which started from the aromatic and basic amino acid derived $\Psi[\mathrm{CH}(\mathrm{CN}) \mathrm{NH}]$ pseudodipeptides $\mathbf{E}$. This scheme consist of reduction of the cyano group, with subsequent in situ lactamization to give the 2oxopiperazines $\mathbf{D}$, followed by selective functionalization at the $\mathrm{N}_{4}(\mathbf{C})$ and $\mathrm{N}_{1}$ positions, and at the exocyclic moiety at position $\mathrm{C}_{5}\left(\mathrm{R}^{4}\right)$. Phenylalanine and tryptophan were selected as aromatic amino acids and lysine and arginine as basic amino acids.

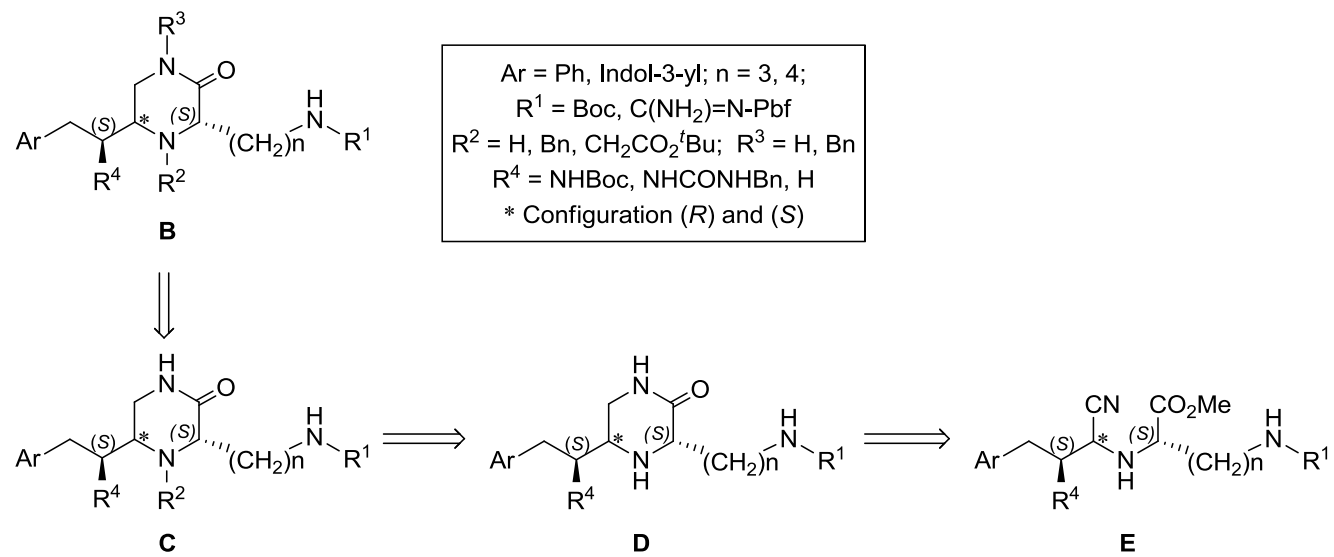

Scheme 1. Retrosynthesis of the 2-oxopiperazines B.

The starting pseudodipeptides $\mathbf{4 a , b}$ and $\mathbf{5 a}$ (Scheme 2) were obtained by applying our methodology for the synthesis of $\Psi[\mathrm{CH}(\mathrm{CN}) \mathrm{NH}]$ pseudodipeptides [51], which involves a modified Strecker reaction of the protected $\alpha$-amino aldehydes Boc-Trp(Boc)-H (1) and BocPhe-H (2) with the methyl esters of the side chain protected basic $\alpha$-amino acids $\mathbf{3 a}$ and $\mathbf{3 b}$ at $-20^{\circ} \mathrm{C}$ for $1 \mathrm{~h}$, followed by in situ reaction with TMSCN at $0{ }^{\circ} \mathrm{C}$ for $24 \mathrm{~h}$. In this way, $4 \mathbf{a}, \mathbf{b}$ and 5a were obtained as $(R S)$-epimeric mixtures at the cyano-bearing stereocenter, which could not be chromatographically resolved. In the tryptophan derivatives $(\boldsymbol{R S})-\mathbf{4 a}, \mathbf{b}$, the $(R) /(S)$ epimer ratio was $(1: 2)$, while in the phenylalanine derivatives $(\boldsymbol{R S})-\mathbf{5 a}$, this ratio was (1:1). The Ni Raney catalyzed hydrogenation of these epimeric mixtures at room temperature and 1 atm of $\mathrm{H}_{2}$ pressure, with in situ lactamization, led to the corresponding 2oxopiperazines $(\boldsymbol{R S})-\mathbf{6 a}, \mathbf{b}$ and $-\mathbf{7 a}$ in $60-80 \%$ overall yield, which were chromatographically resolved in the respective $(R)$ - and $(S)$-epimers. 


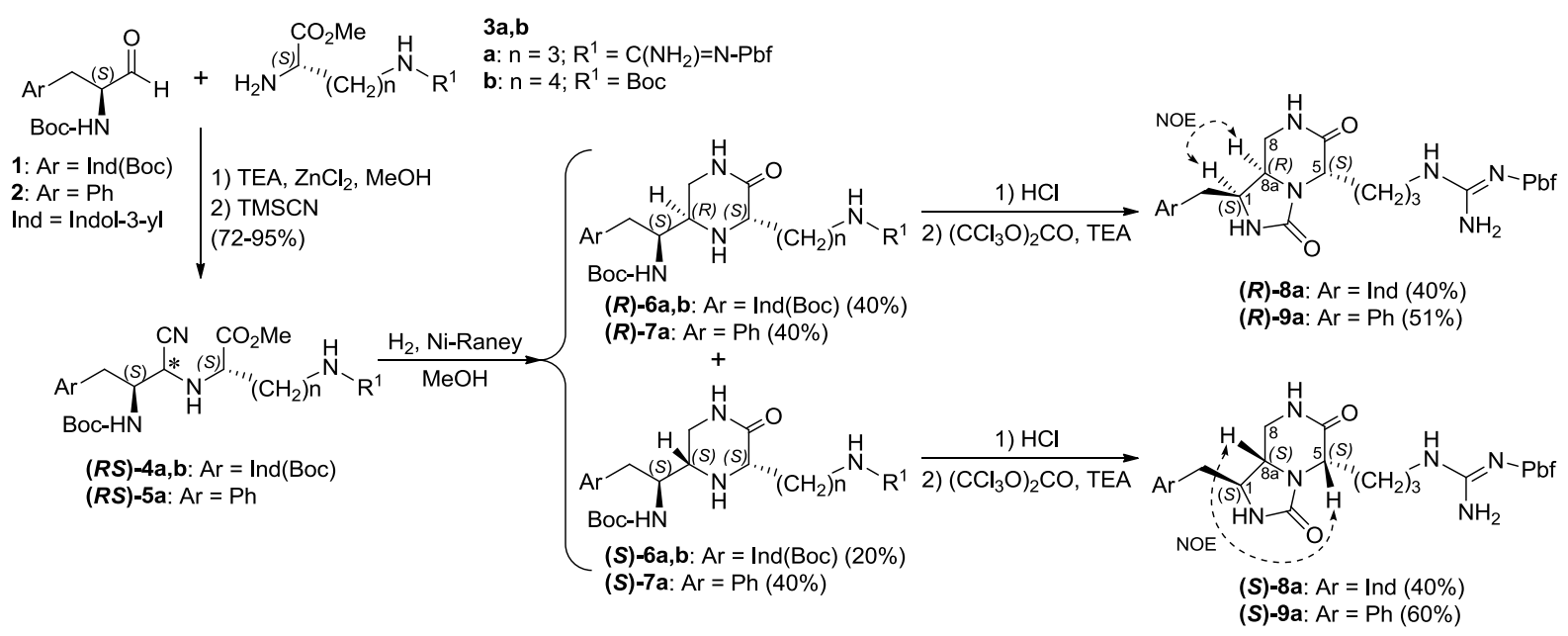

Scheme 2. Synthesis and configuration assignment of the 2-oxopiperazine derivatives $\mathbf{6 a}, \mathbf{b}$ and $7 \mathbf{a}$.

Signal overlapping of the 3-H and 5-H protons in the ${ }^{1} \mathrm{H}$ NMR spectra of $(\boldsymbol{R})$ - and $(\boldsymbol{S})-\mathbf{6 a}, \mathbf{b}$ and -7a did not allow the NOE-based assignment of relative configuration at $\mathrm{C}_{5}$ with respect to that at $\mathrm{C}_{3}$. As the $\mathrm{C}_{3}$ chiral center came from the starting basic amino acid, its configuration was known. Therefore, with the aim of ascertaining the $\mathrm{C}_{5}$ configuration, the Arg derivatives $(\boldsymbol{R})$ - and $(\boldsymbol{S})-\mathbf{6 a}$ and $\mathbf{- 7 \mathbf { a }}$ were transformed into the corresponding 3,6dioxooctahydroimidazo[1,5-a]pyrazine derivatives $(\boldsymbol{R})$ - and $(\boldsymbol{S})-\mathbf{8 a}$ and $-\mathbf{9 a}$ by Boc-removal, followed by reaction with bis(trichloromethyl)carbonate in the presence of TEA. As shown in Scheme 2, the NOE effect between 1-H and 8a-H protons observed in the NOESY 1D spectra of $(\boldsymbol{R})-8 \mathbf{a}$ and $-9 \mathbf{a}$ were indicative of a relative cis disposition and, therefore, a $(R)$ configuration at $\mathrm{C}_{8 \mathrm{a}}$, and, therefore, a $(R)$-configuration at $\mathrm{C}_{5}$ in $(\boldsymbol{R})-\mathbf{6 a}$ and -7a. Similarly, the NOE effect between 5-H and 8a-H observed in the NOESY 1D spectra of $(\boldsymbol{S})$-8a and -9a were indicative of a trans relative disposition, and a $(S)$-configuration at $\mathrm{C}_{8 \mathrm{a}}$ in these compounds, and at $\mathrm{C}_{5}$ in $(\boldsymbol{S})-\mathbf{6 a}$ and $-\mathbf{7 a}$. The comparison of the ${ }^{1} \mathrm{H}$ NMR data of each pair of epimers of these Arg derived 2-oxopiperazines 6a and 7a showed that in the $(R)$-epimers both 6-H were anisochronous $(\Delta \delta \neq 0 \mathrm{ppm})$, while in the $(S)$ they were isochronous $(\Delta \delta=0 \mathrm{ppm})$. Furthermore, 5-H appeared at a lower field (0.05-0.07 ppm) in the $(R)$-epimers than in the $(S)$. On the other hand, it was observed that $\mathrm{C}_{3}$ and $\mathrm{C}_{5}$ appeared 2.5-3.8 ppm at higher field in the $(R)$-epimers than in the $(S)$ in the ${ }^{13} \mathrm{C}$ NMR spectra. These differences between epimers were used for the tentative configuration assignment in the lysine derived 2-oxopiperazines $\mathbf{6 b}$, 
where the selective transformation into 3,6-dioxooctahydroimidazo[1,5- $a$ ]pyrazine derivatives without removal of the Boc group at the side chain was not possible.

The regioselective functionalization of the $\mathrm{N}_{4}$ and $\mathrm{N}_{1}$ positions of the 2-oxopiperazine ring of $\mathbf{6 a , b}$ and $\mathbf{7 b}$ was approached through alkylation reactions, by firstly applying the reaction conditions recently set up for related 2-oxopiperazine derivatives [49]. Thus, benzylation of $(\boldsymbol{R})$-7a was attempted by reaction with 1.1 equivalents of benzyl bromide in the presence of $\mathrm{K}_{2} \mathrm{CO}_{3}$ as base, under argon in $\mathrm{CN}_{3} \mathrm{CN}$ solution at $60{ }^{\circ} \mathrm{C}$. After $24 \mathrm{~h}$ of reaction, the HPLCMS analysis of the crude reaction showed $28 \%$ of the 4-benzyl derivative (R)-11a (Scheme 3) along with $72 \%$ of the starting compound $(\boldsymbol{R})-\mathbf{7 a}$ (Table 1, entry 1). An increase of BnBr from the beginning of the reaction led to dirtier reactions. Therefore, to minimize side reactions an additional equivalent of $\mathrm{BnBr}$ and base was added each $24 \mathrm{~h}$. In this way, after 3 days (Table 1, entry 3), 68\% of (R)-11a was obtained. The starting material completely disappeared with 4.1 equivalents of reagents and 4 days of reaction, but, the reaction was significantly dirtier, to give only a $40 \%$ of the 4-benzyl derivative $(\boldsymbol{R})$-11a (entry 4). $\mathrm{NaH}, \mathrm{Cs}_{2} \mathrm{CO}_{3}$ and diisiopropylethylamine (DIEA) were also tried as bases (Table 1, entries 5-10). Both NaH, $\mathrm{Cs}_{2} \mathrm{CO}_{3}$ led to very dirty reaction crudes. However, with DIEA the reactions were cleaner than with $\mathrm{K}_{2} \mathrm{CO}_{3}$, to provide $85 \%$ of $(\boldsymbol{R})$-11a by adding 4.1 equivalents of reagents (entry 10 ). These reaction conditions were also applied to the $\mathrm{N}_{4}$-benzylation of the 2-oxopiperazines (S)-7a and 6a,b, as well as for their $\mathrm{N}_{4}$-alkylation with tert-butyl bromoacetate (Scheme 3). In general, $(R)$-epimers gave higher yields $(65-80 \%)$ than their respective $(S)$-isomer $(50-80 \%)$. In the ${ }^{1} \mathrm{H}$ NMR spectra the 6-H protons appeared as anisochronous, except for the $(R)$-epimer of the tryptophan derived $\mathrm{N}_{4}$-tert-butoxycarbonylmethyl-2-oxopiperazines $(\boldsymbol{R})$-12a,b, where these protons appeared as isochronous, indicating high conformational flexibility.

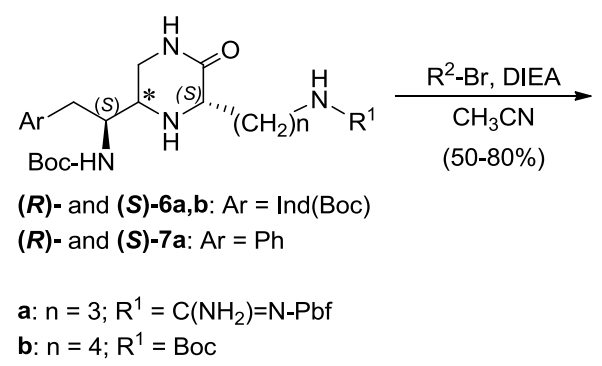

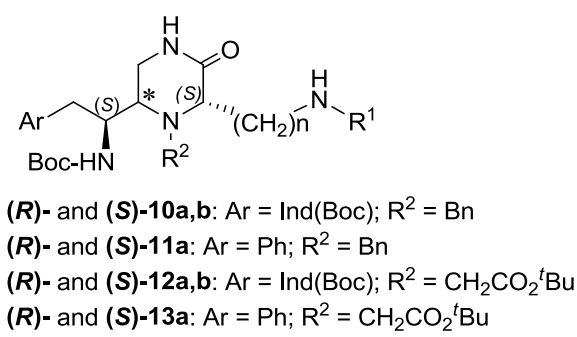

(R)- and (S)-13a: $\mathrm{Ar}=\mathrm{Ph} ; \mathrm{R}^{2}=\mathrm{CH}_{2} \mathrm{CO}_{2}{ }^{t} \mathrm{Bu}$

Scheme 3. $\mathrm{N}_{4}$-Alkylation of the 2-oxopiperazines $\mathbf{6 a , b}$ and $\mathbf{7 a}$. 
Table 1

Optimization of the $\mathrm{N}_{4}$-benzylation conditions for $(\boldsymbol{R})-\mathbf{7 a}$

\begin{tabular}{|c|c|c|c|c|c|}
\hline \multirow{2}{*}{ Entry } & \multirow{2}{*}{ Base } & \multirow{2}{*}{$\mathrm{BnBr}$ and base eq. } & \multirow{2}{*}{$\mathrm{t}$ (days) } & \multicolumn{2}{|c|}{ Yield $(\%)^{a}$} \\
\hline & & & & $(R)-7 \mathbf{a}$ & $(R)-11 \mathrm{a}$ \\
\hline 1 & $\mathrm{~K}_{2} \mathrm{CO}_{3}$ & 1.1 & 1 & 72 & 28 \\
\hline $2^{b}$ & $\mathrm{~K}_{2} \mathrm{CO}_{3}$ & 2.1 & 2 & 40 & 48 \\
\hline $3^{b}$ & $\mathrm{~K}_{2} \mathrm{CO}_{3}$ & 3.1 & 3 & 10 & 68 \\
\hline $4^{b}$ & $\mathrm{~K}_{2} \mathrm{CO}_{3}$ & 4.1 & 4 & 0 & 40 \\
\hline 5 & $\mathrm{NaH}$ & 1.1 & 1 & 47 & 3 \\
\hline 6 & $\mathrm{Cs}_{2} \mathrm{CO}_{3}$ & 1.1 & 1 & 53 & 7 \\
\hline 7 & DIEA & 1.1 & 1 & 70 & 30 \\
\hline $8^{b}$ & DIEA & 2.1 & 2 & 41 & 59 \\
\hline $9^{b}$ & DIEA & 3.1 & 3 & 26 & 74 \\
\hline $10^{b}$ & DIEA & 4.1 & 4 & 0 & 85 \\
\hline
\end{tabular}

After the regiospecific alkylation at the $\mathrm{N}_{4}$-position, benzylation at $\mathrm{N}_{1}$ was studied by using the Phe and Arg derived 2-oxopiperazine $(\boldsymbol{R})$-11a for the setup of reaction conditions. Initially, this benzylation was attempted by applying previously described conditions developed for 2-oxopiperazine analogues [49], that is $\mathrm{MW}$-activated reaction with $\mathrm{BnBr}$ in $\mathrm{CH}_{3} \mathrm{CN}$ at $150{ }^{\circ} \mathrm{C}$, using $\mathrm{Cs}_{2} \mathrm{CO}_{3}$ as base (Table 2, entry 1). This attempt revealed the simultaneous alkylation at the guanidino group of arginine, in spite of being protected with the Pbf group. Thus, as shown in Scheme 4, the HPLC-MS analysis of the crude reaction mixture showed the formation of three products, of which that benzylated at $\mathrm{N}_{1}$ and at the guanidino group $(\boldsymbol{R})-19 a$ (33\%) was the major product. In view of this result, we decided to optimize the selective alkylation at $\mathrm{N}_{1}$ by minimizing the alkylation at the guanidino group. Different solvents, bases, reagent stoichiometries, temperatures and reaction times were tried, whose results are summarized in Table 2. It was not possible to completely avoid the alkylation at the guanidino group and the best results were obtained when the benzylation was performed in (9:1) THF/DMF mixture at $0{ }^{\circ} \mathrm{C}$, adding 0.5 equivalents of $\mathrm{BnBr}$ and 1 equivalent of $\mathrm{NaH}$ in $1 \mathrm{~h}$ intervals during $3 \mathrm{~h}$. In this way, the product of selective benzylation at $\mathrm{N}_{1}(\boldsymbol{R})-\mathbf{1 7}$ a was isolated in $58 \%$ yield, while, the product of dibenzylation at $\mathrm{N}_{1}$ and at the 
guanidino group $(\boldsymbol{R})-19$ a was isolated in $20 \%$. These reaction conditions were applied to the other $\mathrm{N}_{4}$-sustituted-2-oxopiperazines $(\boldsymbol{R})$ - and $(\boldsymbol{S})$-(10a-13a). Although the products resulting from monobenzylation at the guanidino group $(\boldsymbol{R})$ - and $(S)-(\mathbf{1 5 a}, \mathbf{1 8 a}, \mathbf{2 1 a}$ and 24a) were identified in the HPLC-MS analysis of the crude reaction mixtures, they could not be isolated for their complete characterization.

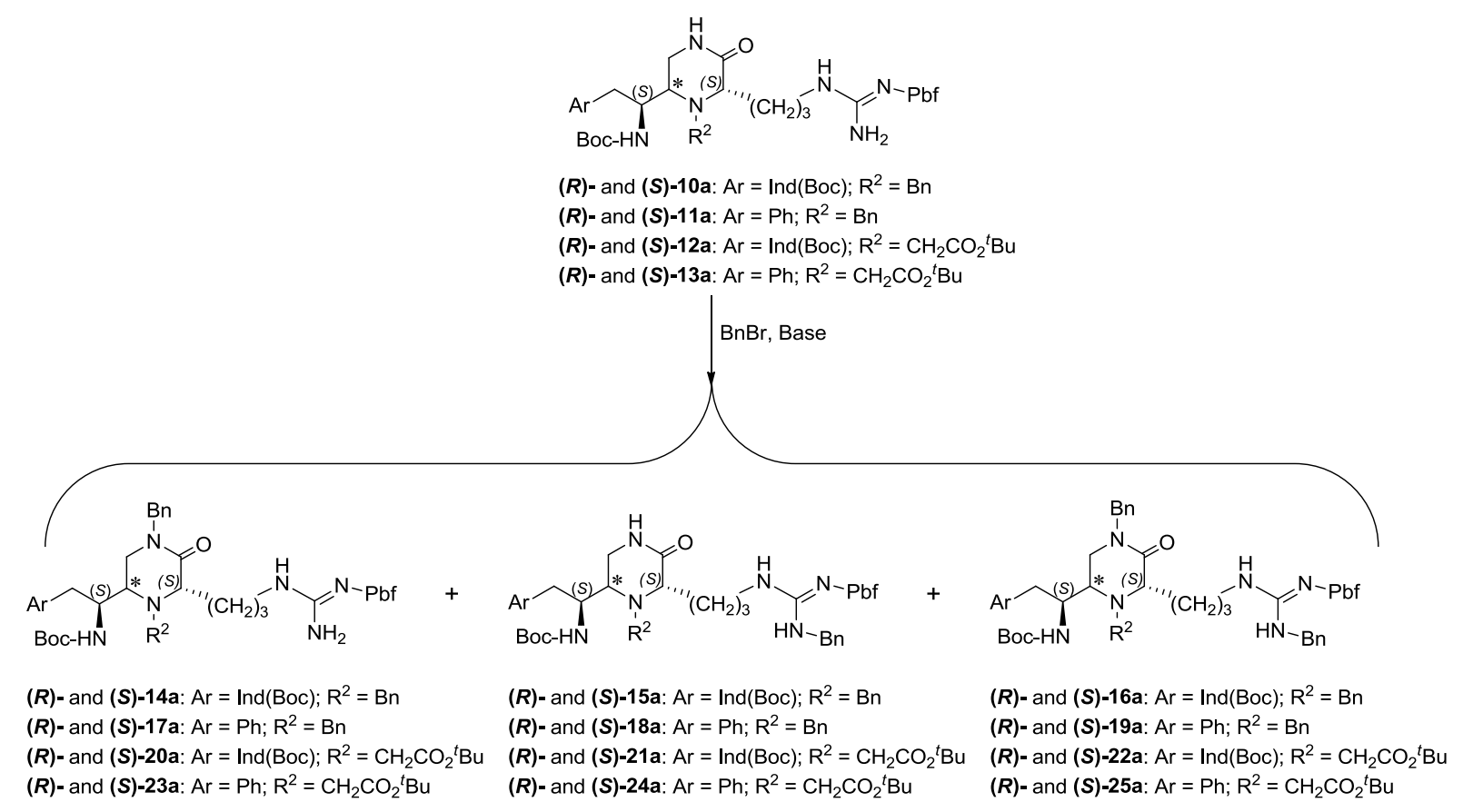

Scheme 4. Benzylation of the arginine derived 4-substituted-2-oxopiperazines 10a-13a.

Table 2

Optimization of the $\mathrm{N}_{1}$-benzylation conditions for $(\boldsymbol{R})$-11a

\begin{tabular}{|c|c|c|c|c|c|c|c|c|}
\hline \multirow{2}{*}{ Entry } & \multirow{2}{*}{ Solvent } & \multirow{2}{*}{ Base (eq.) } & \multirow{2}{*}{$\mathrm{BnBr}$ eq. } & \multirow{2}{*}{$\mathrm{T}\left({ }^{\circ} \mathrm{C}\right)$} & \multirow{2}{*}{$\mathrm{t}(\mathrm{h})$} & \multicolumn{3}{|c|}{ Yield $(\%)^{a}$} \\
\hline & & & & & & $(R)-17 \mathbf{a}$ & $(R)-18 \mathbf{a}$ & $(R)-19 a$ \\
\hline 1 & $\mathrm{CH}_{3} \mathrm{CN}$ & $\mathrm{Cs}_{2} \mathrm{CO}_{3}(1)$ & 1.1 & $150^{b}$ & 0.5 & 5 & 14 & 33 \\
\hline 2 & THF & $\mathrm{NaH}(2)$ & 1.1 & $\mathrm{rt}$ & 1 & 14 & 5 & 3 \\
\hline 3 & THF/DMF (9:1) & $\mathrm{NaH}(2)$ & 1.1 & $\mathrm{rt}$ & 1 & 32 & 1 & 30 \\
\hline 4 & THF/DMF (9:1) & $\mathrm{NaH}(2)$ & 1.1 & -20 & 1 & 11 & 0 & 5 \\
\hline 5 & THF/DMF (9:1) & $\mathrm{NaH}(2)$ & 1.1 & 0 & 1 & 48 & 2 & 25 \\
\hline $6^{c}$ & THF/DMF (9:1) & $\mathrm{NaH}(3)$ & 1.5 & 0 & 3 & 58 & 2 & 20 \\
\hline
\end{tabular}

${ }^{a}$ Determined by HPLC-MS analysis [Sunfire $\left.\mathrm{C}_{18}(4.6 \times 50 \mathrm{~mm}, 3.5 \mu \mathrm{m})\right] .{ }^{b} \mathrm{MW}$ heating. ${ }^{c}$ Addition of 0.5 eq. of $\mathrm{BnBr}$ and 1 eq. of $\mathrm{NaH}$ each hour. 
The assignment of the benzylation position was based on ${ }^{1} \mathrm{H},{ }^{1} \mathrm{H}$ COSY and ${ }^{1} \mathrm{H},{ }^{13} \mathrm{C}$ HSQC and $\mathrm{HMBC}$ correlation spectra. The benzyl methylenic protons at $\mathrm{N}_{1}$ in the piperazine ring and at the guanidino group appeared overlapped in ${ }^{1} \mathrm{H}$ NMR and their assignment was carried out based on their HMBC correlation with the lactamic carbon $\mathrm{C}_{2}$, at 170-172 ppm, and with the guanidine carbon at 154-156 ppm, respectively. Furthermore, these methylenic protons at the $\mathrm{N}_{1}$ position appeared as an $\mathrm{AB}$ system, with a geminal coupling constant of 14-15 Hz. However, the methylenic protons at the guanidine group appeared as a doublet or doublet of doublets coupled to a $\mathrm{NH}$, with a coupling constant of 5-6 Hz, which moved varying the spectra temperature, and disappeared after stirring with $\mathrm{D}_{2} \mathrm{O}$. These data helped us to assign the structure of the trisubstituted guanidine group in 16a, 19a, 22a and 25a, with the Pbf protecting group attached to the $\mathrm{C}=\mathrm{N}$ bond, as described for sulfaguanidines [52]. The HPLC-MS chromatograms of these benzylguanidylated compounds showed two overlapped peaks with the same mass in a (2:1) ratio, which could not be resolved. In spite of the signal overlapping and problems of resolution due to dynamic exchange, two isomers in the same ratio were also observed in the ${ }^{1} \mathrm{H}$ NMR spectra of these compounds in $\left(\mathrm{CD}_{3}\right)_{2} \mathrm{CO}$, which were attributed to the interconverting $E$ - and $Z$-isomers at the guanidino group [53, 54]. This interconversion could explain the low resolution of the ${ }^{1}$ HNMR spectra.

The application of the benzylation conditions of entry 6 in Table 2 to the lysine derived $\mathrm{N}_{1^{-}}$ unsubstituted-2-oxopiperazines $(\boldsymbol{R})$ - and $(S)-(\mathbf{1 0 b}$ and $\mathbf{1 2 b})$ led to the corresponding $\mathrm{N}_{1}$ benzyl derivatives $(\boldsymbol{R})$ - and $(\boldsymbol{S})-(\mathbf{2 6 b}$ and $\mathbf{2 7 b})$ (Scheme 5), which were isolated in $70-80 \%$ yields, without observing benzylation at the lysine protected side chain.

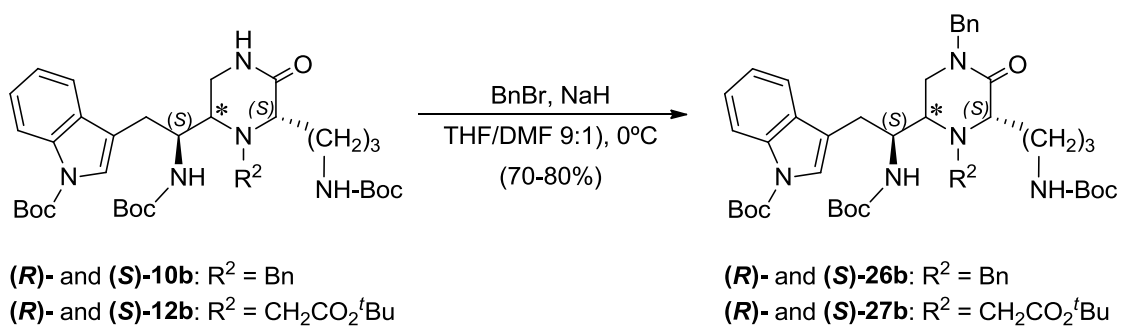

Scheme 5. $\mathrm{N}_{1}$-Benzylation of the lysine derived 4-substituted-2-oxopiperazines $\mathbf{1 0 b}$ and $\mathbf{1 2 b}$. 
In the arginine derived 1,4-dibenzyl-2-oxopiperazines $(\boldsymbol{R})$ - and $(S)-(\mathbf{1 4 a}$ and $\mathbf{1 7 a})$, where the selective removal of the Boc protections without removal of the protection at the basic amino acid side chain was possible, the Boc-HN group of the exocyclic moiety at $\mathrm{C}_{5}$ was replaced by a benzyl-ureido group. Thus, as shown in Scheme 6, removal of the Boc groups, by treatment with $3 \mathrm{~N}$ solution of $\mathrm{HCl}$ in EtOAc, followed by reaction with benzyl isocyanate in the presence of TEA, gave the corresponding ureas $(\boldsymbol{R})$ - and $(\boldsymbol{S})-(\mathbf{2 8 a}$ and $\mathbf{2 9 a})$ in $50-85 \%$ yield. In all cases, the Arg protection Pbf was not affected by the reactions.

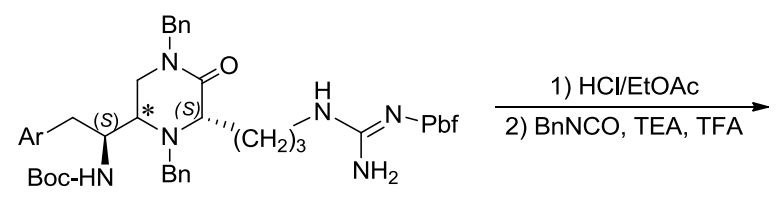

$(R)-$ and $(S)-14 a: A r=\operatorname{lnd}(\mathrm{Boc})$ $(R)-$ and $(S)-17 \mathrm{a}: \mathrm{Ar}=\mathrm{Ph}$

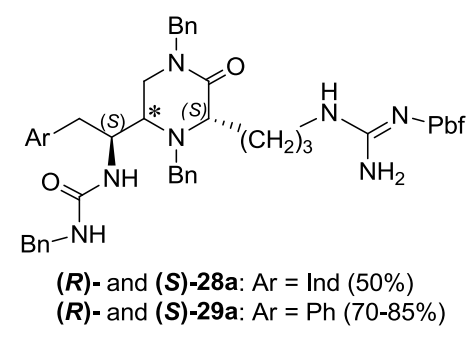

Scheme 6. Synthesis of 2-oxopiperazine derived benzyl-ureas.

It is interesting to note that in the ${ }^{1} \mathrm{H}$ NMR spectra of the $(S)$-epimer of the $\mathrm{N}_{1}$-benzyl- $\mathrm{N}_{4}$ tert-butoxycarbonylmethyl-2-oxopiperazine derivatives 21a, 22a, 24a, 25a and 27b, as well as of the urea derivatives 28a and 29a, the 6-H protons appeared as isochronous, indicative, as above commented, of high conformational flexibility in the piperazine ring.

With the aim of studying the influence of the substituent $R^{4}$ at position 5 of the 2 oxipiperazines upon the biological activity, aralkyl analogues $\left(\mathrm{R}^{4}=\mathrm{H}\right)$ were also synthesized following a similar synthetic strategy to that commented for the tryptophan and phenylalanine derivatives. As in the biological screening of these derivatives, compounds containing the arginine side chain had given better results than those containing the lysine side chain, the new analogues were focused only on those containing arginine. Thus, as shown in Scheme 7, the modified Strecker reaction of aldehydes $\mathbf{3 0}$ and $\mathbf{3 1}$ with $\mathrm{H}-\mathrm{Arg}(\mathrm{Pbf})-\mathrm{OMe}(\mathbf{3 a})$ gave the corresponding epimeric mixtures of $\alpha$-amino nitriles $(\boldsymbol{R S})$-32a and -33a in a (1:1) ratio, which could not be separated. The Ni Raney catalyzed hydrogenation of these $\alpha$-amino nitriles led to the respective 2-oxopiperazine derivatives $(\boldsymbol{R})$ - and $(\boldsymbol{S})$-34a and $\mathbf{- 3 5 a}$, but in yields lower than $40 \%$, due to retro-Strecker and cyano removal side reactions. After studying other catalysts 
and hydrogenation conditions, it was deduced that the best reduction conditions were hydrogen transfer from hydrazine monohydrate using Ni Raney as catalyst under refluxing $\mathrm{MeOH}$ for $10 \mathrm{~min}$ [47]. In this way, the epimeric mixtures $\mathbf{3 4 a}$ and 35a were obtained in 55$86 \%$ yield and were chromatographically resolved into the $(R)$ - and $(S)$-epimers.

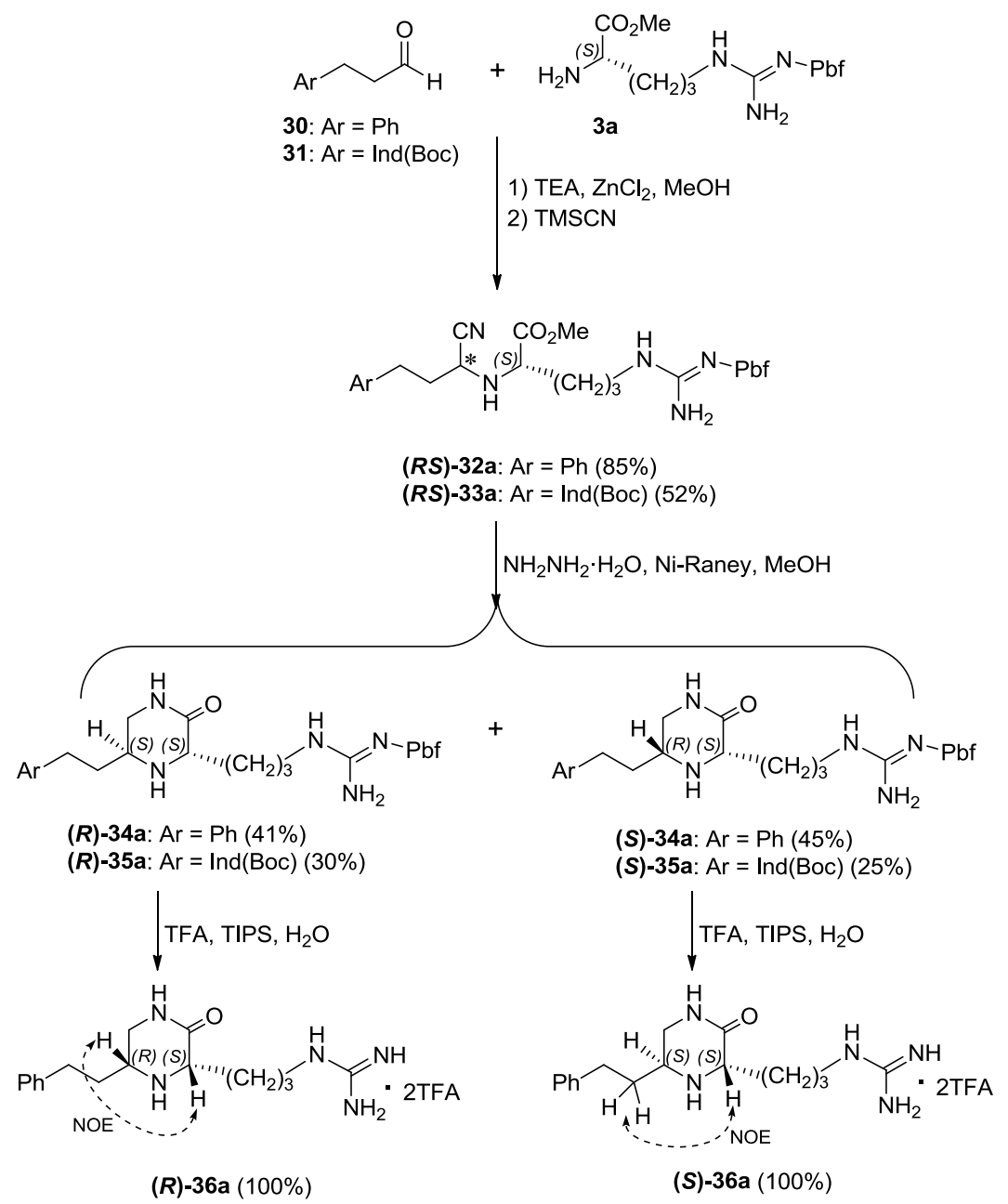

Scheme 7. Synthesis and configuration assignment of the 2-oxopiperazine derivatives 34a and 35a.

NOE effects between the $3-\mathrm{H}$ and $5-\mathrm{H}$ protons were not observed in the NOESY 1D spectra of both epimers of the 2-oxopiperazines 34a and 35a in different solvents $\left[\mathrm{CDCl}_{3}\right.$ or $\left(\mathrm{CD}_{3}\right)_{2} \mathrm{CO}$ ] to be used for assignment of configuration at $\mathrm{C}_{5}$. However, after the removal of the Arg side chain protection Pbf in the 5-phenethyl derivatives 34a, by treatment with TFA in the presence of triisopropylsilane (TIPS), the NOE effects observed in the NOESY 1D spectra of $(\boldsymbol{R})$ - and $(\boldsymbol{S})-36 \mathrm{a}$ in $\mathrm{D}_{2} \mathrm{O}$ [Scheme 7, NOE between $3-\mathrm{H}$ and $5-\mathrm{H}$ in $(\boldsymbol{R})-36 \mathbf{a}$ and 
between 3-H and 5- $\mathrm{CH}_{2}$ in $\left.(\boldsymbol{S})-36 \mathrm{a}\right]$ allowed the indirect assignment of configuration for both epimers in 36a and in 34a. In the comparison of the HPLC-MS and NMR data for the pair of epimers 34a, it was observed that the $(S)$-epimer showed higher $t_{\mathrm{R}}$ than the $(R)$ and that the 3$\mathrm{H}$ proton, as well as $\mathrm{C}_{3}$ and $\mathrm{C}_{5}$, appeared at a lower field in the $(S)$-epimer than in the $(R)$. These differences between the pair of epimers were used for the tentative assignment of the analogue 2-oxopiperazines containing the $N$-Boc-indol-3-yl moiety $\mathbf{3 5 a}$.

The application of the reaction conditions previously set up for the regioselective alkylation of the 2-oxopiperazines 7a to $(\boldsymbol{R})$ - and $(\boldsymbol{S})$-(34a and 35a) allowed first the preparation of the 4-benzyl-2-oxopiperazine derivatives $(\boldsymbol{R})$ - and $(\boldsymbol{S})-(\mathbf{3 7 a}$ and $38 \mathbf{a})$ (Scheme 8), and later that of the 1,4-dibenzyl-2-oxopiperazines $(\boldsymbol{R})$ - and $(\boldsymbol{S})-(\mathbf{3 9 a}$ and $\mathbf{4 2 a})(40-50 \%)$, along with the tribenzylated derivatives $(\boldsymbol{R})$ - and $(\boldsymbol{S})$-(41a and $\mathbf{4 4 a})$, as minor products (814\%). As above commented, these tribenzylated derivatives also appeared as two inseparable mixture of E-and Z-benzylguanidyl derivatives in HPLC-MS and NMR. As in the case of the 2-oxopiperazines 15a, 18a, 21a and 24a, the compounds resulting from benzylation at the $\mathrm{N}_{4}$ position in the piperazine ring and at the guanidine group 40a and 43a, although were detected in the HPLC-MS spectra analyses, they could not be isolated and completely characterized.

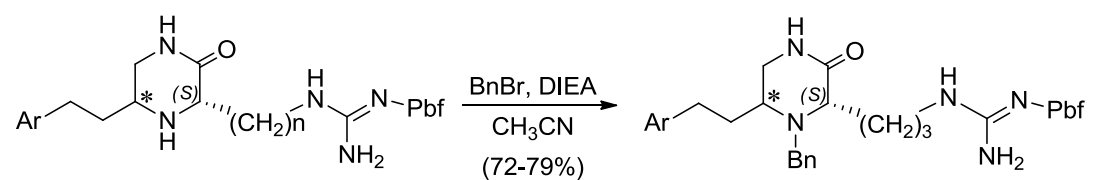

$(R)$-and (S)-34a: $\mathrm{Ar}=\mathrm{Ph}$ $(R)$-and (S)-35a: $\mathrm{Ar}=\operatorname{lnd}(\mathrm{Boc})$
(R)- and (S)-37a: $\mathrm{Ar}=\mathrm{Ph}$ (R)- and (S)-38a: $\mathrm{Ar}=\operatorname{lnd}(\mathrm{Boc})$

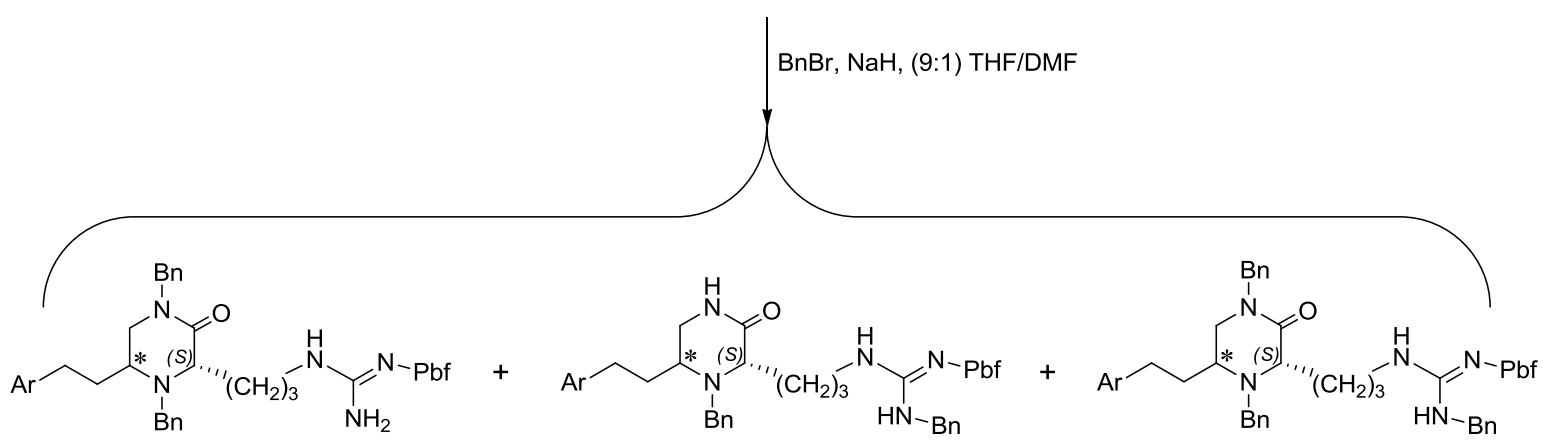

$(R)-$ and (S)-39a: $\mathrm{Ar}=\mathrm{Ph}$

(R)- and (S)-40a: $\mathrm{Ar}=\mathrm{Ph}$ $(R)$ - and (S)-43a: $A r=\operatorname{lnd}(B o c)$

(R)- and (S)-41a: $\mathrm{Ar}=\mathrm{Ph}$ (R)- and (S)-44a: $\mathrm{Ar}=\operatorname{lnd}(\mathrm{Boc})$

Scheme 8. Regioselective benzylations of the 2-oxopiperazines $34 \mathbf{a}$ and $35 \mathbf{a}$. 


\subsection{Biological evaluation}

\subsubsection{PAR1 antagonist activity}

Since PAR1 is mainly expressed in platelets, to evaluate the PAR1 antagonist activity, all new compounds were screened as inhibitors of human platelet aggregation induced by a 30 $\mu \mathrm{M}$ concentration of the PAR1 agonist SFLLRN. The antagonist RWJ-58259 was used as a reference. At a $10 \mu \mathrm{M}$ concentration this antagonist inhibited $98 \%$ the platelet aggregation. All compounds were tested at an initial concentration of $0.1 \mathrm{mg} / \mathrm{mL}(\approx 150 \mu \mathrm{M})$. None of the lysine derived 2-oxopiperazines $6 \mathbf{b}, \mathbf{1 0 b}, \mathbf{1 2 b}, \mathbf{2 6 b}$ and $27 \mathbf{b}$ inhibited the platelet aggregation in this assay. However, as shown in Fig. 2, most of the arginine derived 1-benzyl-4substituted-2-oxopiperazines showed significant $\%$ of inhibition. The low range of variability in the inhibition percentages did not allow to establish clear structure-activity relationships. Thus, the type of aromatic moiety did not significantly affect the inhibition, except for the derivatives containing a benzyl-urea at the exocyclic substituent $\mathrm{R}^{4}$, wherein the Phe derivatives 29a were better than the respective Trp derivatives 28a. In fact, the $(S)$-epimer (S)-29a, with a $57 \%$ of inhibition was the best of the series. Neither the substituent at $\mathrm{N}_{4}\left(\mathrm{R}^{2}\right)$ nor the stereochemistry at $\mathrm{C}_{5}$ showed a clear influence upon the activity. In the case of stereochemistry, its low influence on the activity could be related to the already commented high flexibility of the 2-oxopiperazine ring, observed in the NMR spectra of most compounds. The comparative analysis of the platelet aggregation inhibition results of this series of 2oxopiperazine derivatives of general formula $\mathbf{B}$ with those of our previously reported series of urea analogues A [47], shows that the conformational restriction of the 2-oxopiperazine ring does not affect the binding of the compounds to PAR1, as the best compounds of both series displayed similar activity values. 


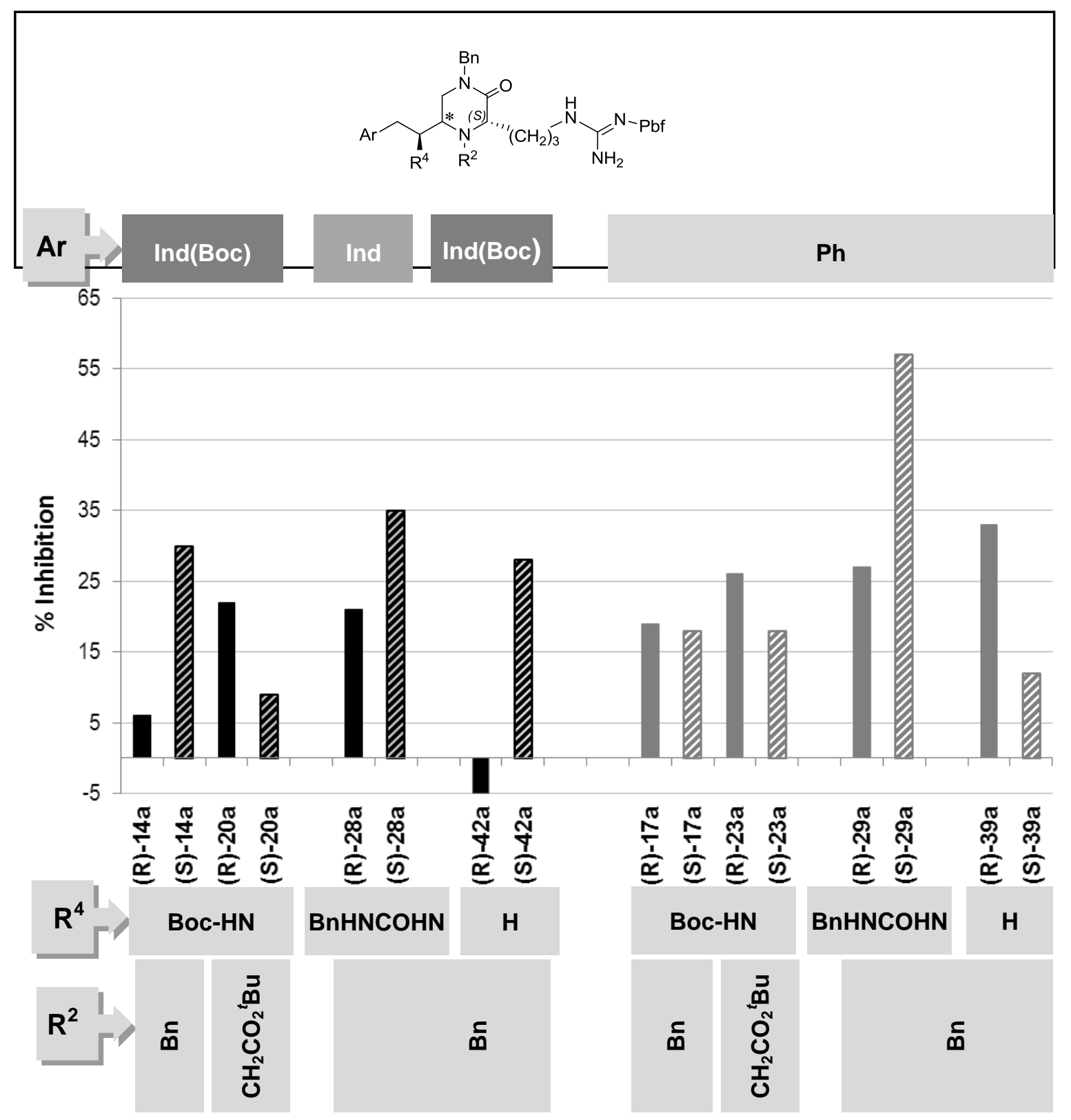

Fig. 2. Inhibition (\%) of human platelet aggregation induced by a $30 \mu \mathrm{M}$ concentration of SFLLRN.

\subsubsection{Antitumor activity}

All new compounds herein described were evaluated as antitumor agents in a HTS programme, which included evaluation of cytotoxicity on three representative human cancer cell lines: breast (MDA-MB-231), lung (A549), and colon (HT-29), using doxorubicin as 
positive control and according to the National Cancer Institute (NCI) protocols. The three cell growth parameters: $\mathrm{GI}_{50}$ (concentration that produces $50 \%$ growth inhibition), TGI (concentration that produces total growth inhibition), and $\mathrm{LC}_{50}$ (concentration that produces $50 \%$ of cellular death) were determined from the data analysis, automatically generated by the HTS laboratory information management system. The $\mathrm{GI}_{50}$ results of the compounds that displayed values lower than the highest $100 \mu \mathrm{M}$ concentration are shown in Table 3 .

\section{Table 3}

$\mathrm{GI}_{50}$ values of the cytotoxicity screening

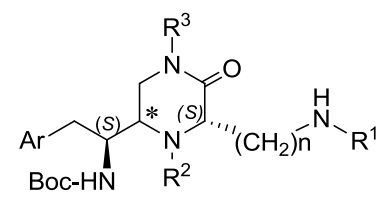

\begin{tabular}{|c|c|c|c|c|c|c|c|c|c|}
\hline \multirow[b]{2}{*}{ Compound } & \multirow[b]{2}{*}{$\mathrm{Ar}$} & \multirow{2}{*}{$\begin{array}{c}\text { Conf. } \\
(*)\end{array}$} & \multirow[b]{2}{*}{$\mathrm{R}^{1}$} & \multirow[b]{2}{*}{$\mathrm{n}$} & \multirow[b]{2}{*}{$\mathrm{R}^{2}$} & \multirow[b]{2}{*}{$\mathrm{R}^{3}$} & \multicolumn{3}{|c|}{$\mathrm{GI}_{50}(\mu \mathrm{M})$} \\
\hline & & & & & & & $\begin{array}{c}\text { Breast } \\
\text { MDAMB-231 }\end{array}$ & $\begin{array}{l}\text { Lung } \\
\text { A549 }\end{array}$ & $\begin{array}{l}\text { Colon } \\
\text { HT-29 }\end{array}$ \\
\hline$(R)-10 \mathbf{a}$ & $\operatorname{Ind}(\mathrm{Boc})$ & $(R)$ & $\mathrm{C}\left(\mathrm{NH}_{2}\right)=\mathrm{N}-\mathrm{Pbf}$ & 3 & $\mathrm{Bn}$ & $\mathrm{H}$ & 4.44 & 4.89 & 3.78 \\
\hline$(S)-10 \mathrm{a}$ & Ind(Boc) & $(S)$ & $\mathrm{C}\left(\mathrm{NH}_{2}\right)=\mathrm{N}-\mathrm{Pbf}$ & 3 & $\mathrm{Bn}$ & $\mathrm{H}$ & 4.55 & 5.55 & 3.78 \\
\hline$(R)-11 \mathrm{a}$ & $\mathrm{Ph}$ & $(R)$ & $\mathrm{C}\left(\mathrm{NH}_{2}\right)=\mathrm{N}-\mathrm{Pbf}$ & 3 & $\mathrm{Bn}$ & $\mathrm{H}$ & 6.83 & 1.24 & 5.26 \\
\hline$(S)-11 \mathrm{a}$ & $\mathrm{Ph}$ & $(S)$ & $\mathrm{C}\left(\mathrm{NH}_{2}\right)=\mathrm{N}-\mathrm{Pbf}$ & 3 & $\mathrm{Bn}$ & $\mathrm{H}$ & 6.04 & 5.39 & 3.29 \\
\hline$(R)-12 a$ & $\operatorname{Ind}(\mathrm{Boc})$ & $(R)$ & $\mathrm{C}\left(\mathrm{NH}_{2}\right)=\mathrm{N}-\mathrm{Pbf}$ & 3 & $\mathrm{CH}_{2} \mathrm{CO}_{2}{ }^{t} \mathrm{Bu}$ & $\mathrm{H}$ & 4.54 & 3.25 & 4.98 \\
\hline$(S)-12 \mathrm{a}$ & $\operatorname{Ind}(\mathrm{Boc})$ & $(S)$ & $\mathrm{C}\left(\mathrm{NH}_{2}\right)=\mathrm{N}-\mathrm{Pbf}$ & 3 & $\mathrm{CH}_{2} \mathrm{CO}_{2}{ }^{t} \mathrm{Bu}$ & $\mathrm{H}$ & 4.87 & 7.68 & 2.92 \\
\hline$(R)-6 b$ & $\operatorname{Ind}(\mathrm{Boc})$ & $(R)$ & Boc & 4 & $\mathrm{H}$ & $\mathrm{H}$ & 5.56 & $>10$ & 5.43 \\
\hline$(S)-\mathbf{6 b}$ & $\operatorname{Ind}(B o c)$ & $(S)$ & Boc & 4 & $\mathrm{H}$ & $\mathrm{H}$ & 5.31 & 9.75 & 5.43 \\
\hline$(R)-10 b$ & $\operatorname{Ind}(\mathrm{Boc})$ & $(R)$ & Boc & 4 & $\mathrm{Bn}$ & $\mathrm{H}$ & 2.92 & 4.86 & 4.58 \\
\hline$(S)-10 \mathrm{~b}$ & $\operatorname{Ind}(\mathrm{Boc})$ & $(S)$ & Boc & 4 & $\mathrm{Bn}$ & $\mathrm{H}$ & 4.58 & 5.56 & 4.45 \\
\hline$(R)-12 b$ & $\operatorname{Ind}(\mathrm{Boc})$ & $(R)$ & Boc & 4 & $\mathrm{CH}_{2} \mathrm{CO}_{2}{ }^{t} \mathrm{Bu}$ & $\mathrm{H}$ & 2.29 & 4.57 & 3.09 \\
\hline$(S)-12 \mathrm{~b}$ & $\operatorname{Ind}(\mathrm{Boc})$ & $(S)$ & Boc & 4 & $\mathrm{CH}_{2} \mathrm{CO}_{2}{ }^{t} \mathrm{Bu}$ & $\mathrm{H}$ & 6.72 & 8.60 & 5.78 \\
\hline$(R)-26 b$ & $\operatorname{Ind}(\mathrm{Boc})$ & $(R)$ & Boc & 4 & $\mathrm{Bn}$ & $\mathrm{Bn}$ & 3.33 & $>10$ & $>10$ \\
\hline$(S)-26 \mathrm{~b}$ & $\operatorname{Ind}(\mathrm{Boc})$ & $(S)$ & Boc & 4 & $\mathrm{Bn}$ & $\mathrm{Bn}$ & 1.48 & $>10$ & 3.83 \\
\hline$(R)-27 b$ & $\operatorname{Ind}(\mathrm{Boc})$ & $(R)$ & Boc & 4 & $\mathrm{CH}_{2} \mathrm{CO}_{2}{ }^{t} \mathrm{Bu}$ & $\mathrm{Bn}$ & 1.56 & $>10$ & 4.44 \\
\hline Doxorubicin $^{a}$ & & & & & & & 0.09 & 0.07 & 0.10 \\
\hline
\end{tabular}

${ }^{a}$ Reference drug 
As shown in Table 3, the $\mathrm{N}_{1}$-unsubstituted arginine derivatives $\left(\mathrm{R}^{3}=\mathrm{H}\right) \mathbf{1 0 a}-\mathbf{1 2} \mathbf{a}$, as well as most of the tryptophan-lysine pseudodipeptide derived 2-oxopiperazines, displayed $\mu \mathrm{M}$ cytotoxicity in the three cell lines. None of the compounds without the Boc-NH group at the aralkyl moiety at $\mathrm{C}_{5}$ showed cytotoxicity at the highest assayed concentration of $100 \mu \mathrm{M}$. Among the cytotoxic compounds, it is noteworthy to comment the selectivity of the lysine derived 1-benzyl-2-oxopiperazines $(\boldsymbol{R})$-, $(S)$-26b and $(\boldsymbol{R})$-27b against breast cancer cells MDAMB-231, for which they were the best compounds of the series. There was not any coincidence between the cytotoxic compounds with those active in the platelet aggregation assay. The lack of correlation between both two types of activities in this series of compounds seems to indicate independent mechanisms of action.

\section{Conclusions}

A series of pseudodipeptide based chiral 1,3,4,5-tetrasubstituted-2-oxopiperazines has been designed and synthesized as potential PAR1 antagonists by applying a DOS strategy. These highly functionalized piperazine derivatives are prepared through a versatile scheme in four steps from aromatic and basic amino acid derived $\Psi[\mathrm{CH}(\mathrm{CN}) \mathrm{NH}]$ pseudodipeptides. This route involves reduction of the cyano group to build the 2-oxo-piperazine ring, followed by selective functionalization at the $\mathrm{N}_{4^{-}}, \mathrm{N}_{1}$-positions, and at the exocyclic moiety at position $\mathrm{C}_{5}$. The selective functionalization at $\mathrm{N}_{4^{-}}$and $\mathrm{N}_{1}$-positions, via alkylation, has required the fine tuning of the reaction conditions, particularly those of alkylation at $\mathrm{N}_{1}$-position in the arginine derivatives, in order to minimize the simultaneous alkylation at the protected arginine guanidino group. In spite of the wide screening of reaction conditions, it has not been possible to completely avoid this side reaction.

To evaluate the PAR1 antagonist activity, all new synthesized compounds have been screened as inhibitors of human platelet aggregation induced by the PAR1 agonist SFLLRN. In this screening, most of the arginine derived 1-benzyl-4-substituted-2-oxopiperazines showed moderate antiaggregant activity. The low range of variability in the platelet inhibition percentages does not allow to establish clear structure-activity relationships. Besides, taking into account our interest in PAR1 antagonists in the field of antitumor agents, all compounds have also been included in a HTS programme for that activity. In this screening some of the $\mathrm{N}_{1}$-unsubstituted arginine derivatives and most of the tryptophan-lysine pseudodipeptide 
derived 2-oxopiperazines, displayed $\mu \mathrm{M}$ cytotoxicity in breast, lung, and colon human cancer cell lines. There is no correlation between the results of PAR1 antagonism and cytotoxicity of the compounds. Therefore, in this series of compounds the mechanisms responsible of both types of activity seems to be independent.

\section{Experimental}

\subsection{General}

All reagents were of commercial quality. Solvents were dried and purified by standard methods. Analytical TLC was performed on aluminum sheets coated with a $0.2 \mathrm{~mm}$ layer of silica gel $60 \mathrm{~F}_{254}$. Silica gel 60 (230-400 mesh) was used for flash chromatography. Analytical RP-HPLC was performed on a Sunfire $C_{18}(4.6 \times 150 \mathrm{~mm}, 3.5 \mu \mathrm{m})$ column, with a flow rate of $1 \mathrm{~mL} / \mathrm{min}$, using a tunable UV detector set at 214 and $254 \mathrm{~nm}$ and gradients of $\mathrm{CH}_{3} \mathrm{CN}$ (solvent A) and $0.05 \%$ TFA in $\mathrm{H}_{2} \mathrm{O}$ (solvent B) as mobile phase. HPLC-MS was performed on a Sunfire $\mathrm{C}_{18}(4.6 \times 50 \mathrm{~mm}, 3.5 \mu \mathrm{m})$ column at $30^{\circ} \mathrm{C}$, with a flow rate of $1 \mathrm{~mL} / \mathrm{min}$. Gradients of $\mathrm{CH}_{3} \mathrm{CN}$ with $0.08 \%$ of formic acid (solvent $\mathrm{A}$ ) in $0.1 \%$ of formic acid in $\mathrm{H}_{2} \mathrm{O}$ (solvent B) were used as mobile phase. Electrospray in positive mode was used for ionization. Melting points were taken on a Mettler Toledo M170 apparatus and are uncorrected. Elemental analyses were obtained on a $\mathrm{CH}-\mathrm{O}-\mathrm{RAOID}$ apparatus. Optical rotations were determined in a Perkin Elmer 141 polarimeter. NMR spectra were recorded using Varian Inova 300, Varian Inova or Mercury 400, and Varian Unity 500 spectrometers. NMR spectra assignment was based on COSY, HSQC, and HMBC spectra. NOESY 1D spectra were carried out at $400 \mathrm{MHz}$, using a gradient duration of $0.0005 \mathrm{~s}$ and mixing times of $0.5 \mathrm{~s}$ and 0.70 s. MW experiments were performed in a MW reactor Emrys ${ }^{\mathrm{TM}}$ Synthesizer (Biotage $\mathrm{AB})$.

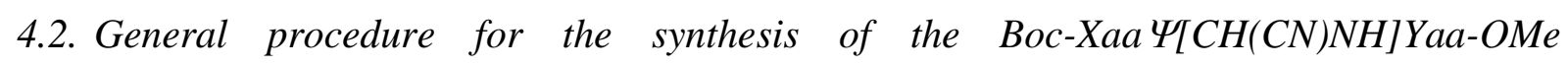
pseudodipeptides (RS)-4a,b and $\mathbf{- 5 a}$

TEA $(1.1 \mathrm{~mL}, 7.96 \mathrm{mmol})$ was added to a solution of $\mathrm{H}$-Lys(Boc)-OMe. $\mathrm{HCl}$ or $\mathrm{H}$ $\mathrm{Arg}(\mathrm{Pbf})-\mathrm{OMe} \cdot \mathrm{HCl}(7.96 \mathrm{mmol})$ in $\mathrm{MeOH}(40 \mathrm{~mL})$. After $15 \mathrm{~min}$ of stirring at room temperature, the mixture was cooled to $-20{ }^{\circ} \mathrm{C}$ and $\mathrm{ZnCl}_{2}(542 \mathrm{mg}, 3.98 \mathrm{mmol})$ was added, followed by the addition of the corresponding aldehyde Boc-Xaa-H (Xaa = Phe [55] or $\operatorname{Trp}\left(\right.$ Boc) [56]) (992 mg, $3.98 \mathrm{mmol}$ ). The solution was stirred for $1 \mathrm{~h}$ at $-20{ }^{\circ} \mathrm{C}$, and the 
reaction was heated to $0{ }^{\circ} \mathrm{C}$. Then, TMSCN was added $(896 \mu \mathrm{L}, 7.16 \mathrm{mmol})$ and the mixture was stirred at $0^{\circ} \mathrm{C}$ during $24 \mathrm{~h}$. The solvent was removed under reduced pressure and the residue was dissolved in EtOAc $(50 \mathrm{~mL})$. The solution was washed with $\mathrm{H}_{2} \mathrm{O}(2 \times 25 \mathrm{~mL})$ and brine $(25 \mathrm{~mL})$, dried over $\mathrm{Na}_{2} \mathrm{SO}_{4}$, and evaporated to dryness. The residue was purified by flash chromatography, using EtOAc in hexane gradient as mobile phase to obtain the epimeric mixtures of pseudodipeptides $(\boldsymbol{R S})-\mathbf{4 a},(\boldsymbol{R S})-\mathbf{4 b}$ and $(\boldsymbol{R S})-\mathbf{5 a}$.

\subsubsection{Boc-Trp(Boc) $\Psi[C H(C N) N H] A r g(P b f)-O M e[(\mathbf{R S})-4 a]$}

Foam $[4.80 \mathrm{~g}, 72 \%,(R: S)=(1: 2)]$; HPLC [Sunfire $\mathrm{C}_{18}(4.6 \times 150 \mathrm{~mm}, 3.5 \mu \mathrm{m}), 60-100 \%$ gradient of solvent A in B, $30 \mathrm{~min}] t_{\mathrm{R}} 14.24 \mathrm{~min} ;{ }^{1} \mathrm{H}$ NMR [400 MHz, $\left(\mathrm{CD}_{3}\right)_{2} \mathrm{CO}$ ]. (R)-4a $\delta$ (ppm): 1.34 (s, 9H, Boc), 1.43 [s, 6H, 2CH $(\mathrm{Pbf})], 1.67$ [s, 9H, Boc (Ind)], 1.68 [m, 2H, $\gamma-\mathrm{H}$ (Arg)], 1.79 [m, 2H, $\beta-\mathrm{H}(\mathrm{Arg})], 2.04$ [s, 3H, $\left.\mathrm{CH}_{3}(\mathrm{Pbf})\right], 2.50$ [s, 3H, $\left.\mathrm{CH}_{3}(\mathrm{Pbf})\right], 2.58$ [s, 3H, $\left.\mathrm{CH}_{3}(\mathrm{Pbf})\right], 2.84(\mathrm{~m}, 1 \mathrm{H}, \mathrm{NH}), 2.97$ [s, 2H, $\left.\mathrm{CH}_{2}(\mathrm{Pbf})\right], 3.04(\mathrm{~m}, 1 \mathrm{H}, 3-\mathrm{H}), 3.24$ [m, 2H, $\delta-\mathrm{H}$ (Arg)], $3.28(\mathrm{~m}, 1 \mathrm{H}, 3-\mathrm{H}), 3.58$ [m, 1H, $\alpha-\mathrm{H}$ (Arg)], 3.68 (s, 3H, OMe), 4.01 (m, 1H, 1-H), $4.18(\mathrm{~m}, 1 \mathrm{H}, 2-\mathrm{H}), 6.34(\mathrm{~d}, 1 \mathrm{H}, J=9.5 \mathrm{~Hz}, N H \mathrm{Boc}), 6.47\left[\mathrm{~m}, 3 \mathrm{H}, \mathrm{NHC}\left(\mathrm{NH}_{2}\right)=\mathrm{N}\right], 7.25(\mathrm{~d}$, $1 \mathrm{H}, J=7.5 \mathrm{~Hz}$, Ind), 7.32 (d, 1H, $J=7.5 \mathrm{~Hz}$, Ind), 7.58 (m, 1H, Ind), 7.62 (d, 1H, $J=7.5 \mathrm{~Hz}$, Ind), 8.13 (d, 1H, $J=7.5 \mathrm{~Hz}$, Ind). (S)-4a $\delta$ (ppm): 1.34 (s, 9H, Boc), 1.43 [s, 6H, 2CH (Pbf)], 1.67 [s, 9H, Boc (Ind)], 1.68 [m, 2H, $\gamma$-H (Arg)], 1.79 [m, 2H, $\beta-\mathrm{H}$ (Arg)], 2.04 [s, 3H, $\mathrm{CH}_{3}$ (Pbf)], 2.50 [s, 3H, $\left.\mathrm{CH}_{3}(\mathrm{Pbf})\right], 2.58$ [s, 3H, $\left.\mathrm{CH}_{3}(\mathrm{Pbf})\right], 2.75$ (m, 1H, NH), 2.96 [s, 2H, $\left.\mathrm{CH}_{2}(\mathrm{Pbf})\right], 3.04(\mathrm{~m}, 1 \mathrm{H}, 3-\mathrm{H}), 3.24$ [m, 2H, $\left.\delta-\mathrm{H}(\mathrm{Arg})\right], 3.28(\mathrm{~m}, 1 \mathrm{H}, 3-\mathrm{H}), 3.41[\mathrm{~m}, 1 \mathrm{H}, \alpha-\mathrm{H}$ (Arg)], 3.70 (s, 3H, OMe), 3.95 (m, 1H, 1-H), 4.24 (m, 1H, 2-H), 6.44 (m, 1H, NHBoc), 6.47 $\left[\mathrm{m}, 3 \mathrm{H}, \mathrm{NHC}\left(\mathrm{NH}_{2}\right)=\mathrm{N}\right], 7.25(\mathrm{~d}, 1 \mathrm{H}, J=7.5 \mathrm{~Hz}, \mathrm{Ind}), 7.32(\mathrm{~d}, 1 \mathrm{H}, J=7.5 \mathrm{~Hz}, \mathrm{Ind}), 7.58(\mathrm{~m}$, 1H, Ind), 7.62 (d, $1 \mathrm{H}, J=7.5 \mathrm{~Hz}$, Ind), 8.13 (d, $1 \mathrm{H}, J=7.5 \mathrm{~Hz}$, Ind); ${ }^{13} \mathrm{C}$ NMR $(100 \mathrm{MHz}$, $\left.\left(\mathrm{CD}_{3}\right)_{2} \mathrm{CO}\right) .(\boldsymbol{R})-4 \mathbf{a} \delta(\mathrm{ppm}): 12.5,18.1,19.5$ [3CH $\left.3(\mathrm{Pbf})\right], 27.0\left(\mathrm{C}_{3}\right), 27.2\left[\mathrm{C}_{\gamma}(\mathrm{Arg})\right], 28.3$ [3 $\left.3 \mathrm{CH}_{3}(\mathrm{Boc})\right], 28.5\left[2 \mathrm{CH}_{3}(\mathrm{Pbf})\right], 28.7\left[3 \mathrm{CH}_{3}(\mathrm{Boc})\right], 31.0\left[\mathrm{C}_{\beta}(\mathrm{Arg})\right], 41.4\left[\mathrm{C}_{\delta}(\mathrm{Arg})\right], 43.6$ [CH $2(\mathrm{Pbf})], 52.1$ (Ome), $53.3\left(\mathrm{C}_{2}\right), 54.4\left(\mathrm{C}_{1}\right), 59.7$ [ $\left.\mathrm{C}_{\alpha}(\mathrm{Arg})\right], 79.4,84.1$ [2C (2Boc)], 86.9 [C (Pbf)], 115.6 [CH (Ind)], 117.5 [C (Pbf)], 117.6 [C (Ind)], 119.5 (CN), 119.9, 123.3, 124.8, 125.1 [4CH (Ind)], 125.3 [C (Pbf)], 131.6 [C (Ind)], 132.8, 135.6 [2C (Pbf)], 136.3 [C (Ind)], 138.7 [C (Pbf)], 150.2, 156.3 [2CO (2Boc)], 157.3 [C $\left(\mathrm{NHC}\left(\mathrm{NH}_{2}\right)=\mathrm{N}\right)$ ], 158.9 [C (Pbf)], $174.3\left(\mathrm{CO}_{2}\right)$. (S)-4a $\delta(\mathrm{ppm}): 12.5,18.1,19.5\left[3 \mathrm{CH}_{3}(\mathrm{Pbf})\right], 26.9\left(\mathrm{C}_{3}\right), 27.0\left[\mathrm{C}_{\gamma}(\mathrm{Arg})\right], 28.3$ [3 $\left.\mathrm{CH}_{3}(\mathrm{Boc})\right], 28.5\left[2 \mathrm{CH}_{3}(\mathrm{Pbf})\right], 28.7\left[3 \mathrm{CH}_{3}(\mathrm{Boc})\right], 31.2\left[\mathrm{C}_{\beta}(\mathrm{Arg})\right], 41.4\left[\mathrm{C}_{\delta}(\mathrm{Arg})\right], 43.6$ [CH $\left.\mathrm{CH}_{2}(\mathrm{Pbf})\right], 52.0$ (Ome), $53.8\left(\mathrm{C}_{2}\right), 54.0\left(\mathrm{C}_{1}\right), 61.2$ [C $\left.\mathrm{C}_{\alpha}(\mathrm{Arg})\right], 79.4,84.1$ [2C (2Boc)], 86.9 
[C (Pbf)], 115.6 [CH (Ind)], 117.5 [C (Pbf)], 117.7 [C (Ind)], 119.6 (CN), 119.9, 123.3, 124.8, 125.1 [4CH (Ind)], 125.3 [C (Pbf)], 131.4 [C (Ind)], 132.8, 135.6 [2C (Pbf)], 136.3 [C (Ind)], 138.7 [C (Pbf)], 150.2, 156.5 [2CO (2Boc)], 157.3 [C $\left.\left(\mathrm{NHC}\left(\mathrm{NH}_{2}\right)=\mathrm{N}\right)\right], 158.9$ [C (Pbf)], $174.8\left(\mathrm{CO}_{2}\right)$; ES-MS m/z $838.7[\mathrm{M}+1]^{+}$; Anal. calcd. for $\mathrm{C}_{42} \mathrm{H}_{59} \mathrm{~N}_{7} \mathrm{O}_{9} \mathrm{~S}: \mathrm{C}, 60.20 ; \mathrm{H}, 7.10 ; \mathrm{N}$, 11.70. Found: C, 60.11; H, 6.95; N, 11.94 .

\subsubsection{Boc-Trp(Boc) $\Psi[C H(C N) N H] L y s(B o c)-O M e[(\mathbf{R S})-4 b]$}

Foam $[4.60 \mathrm{~g}, 87 \%,(R: S)=(1: 2)]$; HPLC [Sunfire $\mathrm{C}_{18}(4.6 \times 150 \mathrm{~mm}, 3.5 \mu \mathrm{m}), 70-100 \%$ gradient of solvent $\mathrm{A}$ in $\mathrm{B}, 30 \mathrm{~min}] t_{\mathrm{R}} 11.00 \mathrm{~min}[(\boldsymbol{R})-\mathbf{4 b}]$ and $11.36 \mathrm{~min}[(\boldsymbol{S})-\mathbf{4 b}] ;{ }^{1} \mathrm{H} \mathrm{NMR}$ [400 MHz, $\left(\mathrm{CD}_{3}\right)_{2} \mathrm{CO}$ ]. (R)-4b $\delta$ (ppm): 1.35 (s, 9H, Boc), 1.38 [s, 9H, Boc (Lys)], 1.49 [m, 2H, $\gamma-\mathrm{H}$ (Lys)], 1.52 [m, 2H, $\delta$-H (Lys)], 1.67 [m, 9H, Boc (Ind)], 1.81 [m, 2H, $\beta$-H (Lys)], $2.82(\mathrm{~m}, 1 \mathrm{H}, \mathrm{NH}), 3.06$ (m, 1H, 3-H), 3.08 [m, 2H, $\varepsilon-\mathrm{H}$ (Lys)], 3.31 (dd, 1H, $J=3$ and $15 \mathrm{~Hz}$, 3-H), 3.58 [dd, 1H, $J=6.5$ and $12 \mathrm{~Hz}, \alpha-\mathrm{H}$ (Lys)], 3.71 (s, 3H, OMe), 4.02 (m, 1H, 1-H), 4.20 (m, 1H, 2-H), 5.94 [m, 1H, NHBoc (Lys)], 6.34 (d, 1H, J = 9 Hz, NHBoc), 7.26 (m, 1H, Ind), 7.32 (m, 1H, Ind), 7.59 (m, 1H, Ind), 7.64 (d, 1H, $J=7.5 \mathrm{~Hz}$, Ind), 8.14 (d, 1H, J= $7.5 \mathrm{~Hz}$, Ind). (S)-4b $\delta$ (ppm): 1.35 (s, 9H, Boc), 1.38 [s, 9H, Boc (Lys)], 1.49 [m, 2H, $\gamma-\mathrm{H}$ (Lys)], 1.52 [m, 2H, $\delta$-H (Lys)], 1.67 [m, 9H, Boc (Ind)], 1.73 [m, 2H, $\beta$-H (Lys)], 2.74 (m, 1H, NH), 3.01 (m, 1H, 3-H), 3.08 [m, 2H, $\varepsilon-\mathrm{H}$ (Lys)], 3.24 (dd, 1H, $J=4.5$ and $15 \mathrm{~Hz}, 3-\mathrm{H}), 3.40$ [dd, 1H, $J$ $=8$ and $15.5 \mathrm{~Hz}, \alpha-\mathrm{H}$ (Lys)], 3.72 (s, 3H, OMe), 3.98 (m, 1H, 1-H), 4.25 (m, 1H, 2-H), 5.94 [m, 1H, NHBoc (Lys)], 6.45 (d, 1H, $J=8.5 \mathrm{~Hz}, N H B o c), 7.26$ (m, 1H, Ind), 7.32 (m, 1H, Ind), 7.59 (m, 1H, Ind), 7.69 (d, 1H, $J=7.5 \mathrm{~Hz}$, Ind), 8.14 (d, $1 \mathrm{H}, J=7.5 \mathrm{~Hz}$, Ind); ${ }^{13} \mathrm{C} \mathrm{NMR}$ $\left(100 \mathrm{MHz},\left(\mathrm{CD}_{3}\right)_{2} \mathrm{CO}\right) .(\boldsymbol{R})-\mathbf{4 b} \delta(\mathrm{ppm}): 23.6\left[\mathrm{C}_{\gamma}(\mathrm{Lys})\right], 27.3\left(\mathrm{C}_{3}\right), 28.3,28.7$ [9 $\left.\mathrm{CH}_{3}(3 \mathrm{Boc})\right]$, $30.6\left[\mathrm{C}_{\delta}(\mathrm{Lys})\right], 33.5\left[\mathrm{C}_{\beta}(\mathrm{Lys})\right], 40.8\left[\mathrm{C}_{\varepsilon}(\mathrm{Lys})\right], 52.1(\mathrm{OMe}), 53.3\left(\mathrm{C}_{2}\right), 54.5\left(\mathrm{C}_{1}\right), 59.9\left[\mathrm{C}_{\alpha}\right.$ (Lys)], 78.3, 79.3, 84.1 [3C (3Boc)], 115.9 [CH (Ind)], 117.6 [C (Ind)], 119.6 (CN), 119.9, 123.3, 124.8, 125.1 [4CH (Ind)], 131.7, 136.4 [2C (Ind)], 150.3, 156.5 [3CO (3Boc)], 174.5 $\left[\mathrm{CO}_{2}\right] .(\boldsymbol{S})-\mathbf{4 b} \delta(\mathrm{ppm}): 23.6\left[\mathrm{C}_{\gamma}(\mathrm{Lys})\right], 27.0\left(\mathrm{C}_{3}\right), 28.3,28.5$ [9CH $\left.3(3 \mathrm{Boc})\right], 30.6\left[\mathrm{C}_{\delta}(\mathrm{Lys})\right]$, $33.7\left[\mathrm{C}_{\beta}\right.$ (Lys)], 40.9 [C $\left.\mathrm{C}_{\varepsilon}(\mathrm{Lys})\right], 52.0$ [OMe], $53.7\left(\mathrm{C}_{2}\right), 54.0\left(\mathrm{C}_{1}\right), 61.5\left[\mathrm{C}_{\alpha}(\mathrm{Lys})\right], 78.3,79.3$, 84.1 [3C (3Boc)], 115.9 [CH (Ind)], 117.7 [C (Ind)], 119.7 (CN), 119.9, 123.4, 124.8, 125.1 [4CH (Ind)], 131.5, 136.3 [2C (Ind)], 150.3, 156.5 [3CO (3Boc)], $175.0\left(\mathrm{CO}_{2}\right) ; \mathrm{ES}-\mathrm{MS} \mathrm{m} / \mathrm{z}$ $658.8[\mathrm{M}+1]^{+}$; Anal. calcd. for $\mathrm{C}_{34} \mathrm{H}_{51} \mathrm{~N}_{5} \mathrm{O}_{8}: \mathrm{C}, 62.08 ; \mathrm{H}, 7.81 ; \mathrm{N}, 10.65$. Found: $\mathrm{C}, 62.29 ; \mathrm{H}$, 7.67; N, 10.78 .

\subsubsection{Boc-Phe $\Psi[C H(C N) N H] A r g(P b f)-O M e[(\mathbf{R S})-5 a]$}


Foam [5.30 g, 95\%, $(R: S)=(1: 1)]$; HPLC [Sunfire $\mathrm{C}_{18}(4.6 \times 150 \mathrm{~mm}, 3.5 \mu \mathrm{m}), 60-100 \%$ gradient of solvent $\mathrm{A}$ in $30 \mathrm{~min}] t_{\mathrm{R}} 10.05 \mathrm{~min}[(\boldsymbol{R})-\mathbf{5 a}]$ and $10.25 \mathrm{~min}[(\boldsymbol{S})-\mathbf{5 a}] ;{ }^{1} \mathrm{H}$ NMR [400 $\left.\mathrm{MHz},\left(\mathrm{CD}_{3}\right)_{2} \mathrm{CO}\right] .(\boldsymbol{R})-5 \mathrm{a} \delta(\mathrm{ppm}): 1.32$ (s, 9H, Boc), 1.44 [s, 6H, 2CH 3 (Pbf)], 1.66 [m, 2H, $\gamma-\mathrm{H}(\mathrm{Arg})], 1.78$ [m, 2H, $\beta-\mathrm{H}(\mathrm{Arg})], 2.05$ [s, 3H, $\mathrm{CH}_{3}$ (Pbf)], 2.50 [s, 3H, $\mathrm{CH}_{3}$ (Pbf)], 2.59 [s, $3 \mathrm{H}, \mathrm{CH}_{3}$ (Pbf)], 2.63 (m, 1H, 3-H), 2.73 (m, 1H, 3-H), 2.85 (m, 1H, NH), 2.98 [s, 2H, $\mathrm{CH}_{2}$ (Pbf)], 3.24 [m, 2H, $\delta-\mathrm{H}(\mathrm{Arg})], 3.53$ [m, 1H, $\alpha-\mathrm{H}$ (Arg)], 3.66 (s, 3H, OMe), 3.90 (t, 1H, $J=$ $7 \mathrm{~Hz}, 1-\mathrm{H}), 4.08(\mathrm{~m}, 1 \mathrm{H}, 2-\mathrm{H}), 6.29(\mathrm{~d}, 1 \mathrm{H}, J=7.5 \mathrm{~Hz}, N H \mathrm{Boc}), 6.49$ [m, 3H, $\left.\mathrm{NHC}\left(\mathrm{NH}_{2}\right)=\mathrm{N}\right], 7.21(\mathrm{~m}, 1 \mathrm{H}, \mathrm{Ph}), 7.24-7.32(\mathrm{~m}, 4 \mathrm{H}, \mathrm{Ph}) .(\boldsymbol{S})-5 \mathbf{a} \delta(\mathrm{ppm}): 1.32$ (s, 9H, Boc), $1.44\left[\mathrm{~s}, 6 \mathrm{H}, 2 \mathrm{CH}_{3}(\mathrm{Pbf})\right], 1.66[\mathrm{~m}, 2 \mathrm{H}, \gamma-\mathrm{H}(\mathrm{Arg})], 1.78$ [m, 2H, $\left.\beta-\mathrm{H}(\mathrm{Arg})\right], 2.05\left[\mathrm{~s}, 3 \mathrm{H}, \mathrm{CH}_{3}\right.$ (Pbf)], 2.50 [s, 3H, $\left.\mathrm{CH}_{3}(\mathrm{Pbf})\right], 2.59$ [s, 3H, $\mathrm{CH}_{3}(\mathrm{Pbf})$ ], 2.63 (m, 1H, 3-H), 2.73 (m, 1H, 3-H), $2.81(\mathrm{~m}, 1 \mathrm{H}, \mathrm{NH}), 2.98$ [s, 2H, $\left.\mathrm{CH}_{2}(\mathrm{Pbf})\right], 3.24$ [m, 2H, $\left.\delta-\mathrm{H}(\mathrm{Arg})\right], 3.36$ [m, 1H, $\alpha-\mathrm{H}$ (Arg)], $3.69(\mathrm{~s}, 3 \mathrm{H}, \mathrm{OMe}), 3.84(\mathrm{dd}, 1 \mathrm{H}, J=4.5$ and $11 \mathrm{~Hz}, 1-\mathrm{H}), 4.08(\mathrm{~m}, 1 \mathrm{H}, 2-\mathrm{H}), 6.38(\mathrm{~d}, 1 \mathrm{H}, J=$ $9 \mathrm{~Hz}, N H B o c), 6.49\left[\mathrm{~m}, 3 \mathrm{H}, \mathrm{NHC}\left(\mathrm{NH}_{2}\right)=\mathrm{N}\right], 7.21(\mathrm{~m}, 1 \mathrm{H}, \mathrm{Ph}), 7.24-7.32(\mathrm{~m}, 4 \mathrm{H}, \mathrm{Ph}) ;{ }^{13} \mathrm{C}$ NMR (100 MHz, $\left.\left(\mathrm{CD}_{3}\right)_{2} \mathrm{CO}\right) .(\boldsymbol{R})-5 \mathbf{a} \delta(\mathrm{ppm}): 12.5,18.1,19.4$ [3 $\left.\mathrm{CH}_{3}(\mathrm{Pbf})\right], 26.6\left[\mathrm{C}_{\gamma}(\mathrm{Arg})\right]$, $28.5\left[2 \mathrm{CH}_{3}(\mathrm{Pbf})\right], 28.7\left[3 \mathrm{CH}_{3}(\mathrm{Boc})\right], 31.0\left[\mathrm{C}_{\beta}(\mathrm{Arg})\right], 37.3\left(\mathrm{C}_{3}\right), 41.3\left[\mathrm{C}_{\delta}(\mathrm{Arg})\right], 43.6\left[\mathrm{CH}_{2}\right.$ (Pbf)], 52.1 (Ome), $53.8\left(\mathrm{C}_{2}\right), 54.7\left(\mathrm{C}_{1}\right), 59.5$ [C $\left.\mathrm{C}_{\alpha}(\mathrm{Arg})\right], 79.3$ [C (Boc)], 86.6, 117.5 [2C (Pbf)], $119.3(\mathrm{CN}), 125.3$ [C (Pbf)], 127.1, 129.1, 130.8 [5CH (Ph)], 132.8, 135.5, 138.7 [3C $(\mathrm{Pbf})], 139.4$ [C (Ph)], 156.3 [CO (Boc)], 157.9 [C $\left.\left(\mathrm{NHC}\left(\mathrm{NH}_{2}\right)=\mathrm{N}\right)\right], 158.9$ [C (Pbf)], 174.4 $\left[\mathrm{CO}_{2}\right] .(\mathbf{S})-\mathbf{5 a} \delta(\mathrm{ppm}): 12.5,18.1,19.4\left[3 \mathrm{CH}_{3}(\mathrm{Pbf})\right], 26.0\left[\mathrm{C}_{\gamma}(\mathrm{Arg})\right], 28.5\left[2 \mathrm{CH}_{3}(\mathrm{Pbf})\right], 28.7$ [3CH 3 (Boc)], $31.1\left[\mathrm{C}_{\beta}(\mathrm{Arg})\right], 37.1\left(\mathrm{C}_{3}\right), 41.3\left[\mathrm{C}_{\delta}(\mathrm{Arg})\right], 43.6\left[\mathrm{CH}_{2}(\mathrm{Pbf})\right], 52.0(\mathrm{Ome}), 54.5$ $\left(\mathrm{C}_{2}\right), 54.5\left(\mathrm{C}_{1}\right), 60.9\left[\mathrm{C}_{\alpha}(\mathrm{Arg})\right], 79.3[\mathrm{C}(\mathrm{Boc})], 86.6,117.5$ [2C (Pbf)], $119.4(\mathrm{CN}), 125.3$ [C (Pbf)], 127.2, 129.2, 130.8 [5CH (Ph)], 132.8, 135.5, 138.7 [3C (Pbf)], 139.4 [C (Ph)], 156.3 [CO (Boc)], 157.9 [C $\left.\left(\mathrm{NHC}\left(\mathrm{NH}_{2}\right)=\mathrm{N}\right)\right], 158.9$ [C (Pbf)], $174.7\left(\mathrm{CO}_{2}\right)$; ES-MS m/z 699.6 $[\mathrm{M}+1]^{+}$; Anal. calcd. for $\mathrm{C}_{35} \mathrm{H}_{50} \mathrm{~N}_{6} \mathrm{O}_{7} \mathrm{~S}: \mathrm{C}, 60.15 ; \mathrm{H}, 7.21 ; \mathrm{N}, 12.03$. Found: C, 60.21; H, 7.39; $\mathrm{N}, 11.91$.

\subsection{General procedure for the synthesis of the 2-oxopiperazine derivatives ( $\mathbf{R})$ - and (S)-6a,b and $-7 a$}

Raney-Ni (1.50 g) was added to a solution of the corresponding epimeric mixture of pseudodipeptides $(\boldsymbol{R S}) \mathbf{- 4 a , b}$ and $\mathbf{- 5 a}(2.88 \mathrm{mmol})$ in $\mathrm{MeOH}(15 \mathrm{~mL})$ and the mixture was hydrogenated at $1 \mathrm{~atm}$ of $\mathrm{H}_{2}$ at room temperature for $12 \mathrm{~h}$. Afterward, the reaction mixture was filtered over celite and the solvent was evaporated under reduced pressure. The residue 
was purified by flash chromatography, by using $\mathrm{MeOH}$ in $\mathrm{CH}_{2} \mathrm{Cl}_{2}$ gradient as mobile phase to

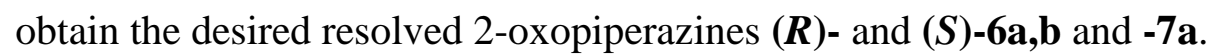

\subsection{1. (5R,3S)-5-((S)-1-((tert-Butoxycarbonyl)amino-2-(1-(tert-butoxycarbonyl)-indol-3-yl)} ethyl-3-(3-(2-((2,2,4,6,7-pentamethyl-2,3-dihydrobenzofuran-5-yl)sulfonyl)guanidino)propyl)-2-oxopiperazine $[(\mathbf{R})-6 a]^{1}$

White solid (933 mg, 40\%); $[\alpha]_{\mathrm{D}}{ }^{20}-5.1\left(c\right.$ 1.0, $\left.\mathrm{CH}_{2} \mathrm{Cl}_{2}\right)$; $\mathrm{Mp}: 118-120{ }^{\circ} \mathrm{C}$ (EtOAc/hexane); HPLC [Sunfire $\mathrm{C}_{18}(4.6 \times 150 \mathrm{~mm}, 3.5 \mu \mathrm{m})$, isocratic $50 \%$ of solvent $\mathrm{A}$ ] $t_{\mathrm{R}} 8.70 \mathrm{~min} ;{ }^{1} \mathrm{H}$ NMR (400 MHz, $\left.\left(\mathrm{CD}_{3}\right)_{2} \mathrm{CO}\right) \delta(\mathrm{ppm}): 1.30$ (s, 9H, Boc), 1.42 [s, 6H, 2CH $\left.3(\mathrm{Pbf})\right], 1.66$ [s, 3H, Boc (Ind)], 1.69 [m, 1H, $\gamma-\mathrm{H}$ (Arg)], 1.73 [m, 1H, $\gamma-\mathrm{H}$ (Arg)], 1.82 [m, 1H, $\beta-\mathrm{H}$ (Arg)], $1.89\left[\mathrm{~m}, 1 \mathrm{H}, \beta-\mathrm{H}\right.$ (Arg)], 2.04 [s, 3H, $\mathrm{CH}_{3}$ (Pbf)], 2.50 [s, 3H, $\mathrm{CH}_{3}$ (Pbf)], 2.59 [s, 3H, $\mathrm{CH}_{3}$ (Pbf)], 2.87 (m, 1H, $\mathrm{CH}_{2}$-Ind), 2.95 [s, 2H, $\mathrm{CH}_{2}$ (Pbf)], 3.19 (m, 1H, 5-H), 3.27 (m, 1H, 6-H), $3.28[\mathrm{~m}, 2 \mathrm{H}, \delta-\mathrm{H}(\mathrm{Arg})], 3.35[\mathrm{~m}, 1 \mathrm{H}, 3-\mathrm{H}], 3.40\left(\mathrm{~m}, 1 \mathrm{H}, \mathrm{CH}_{2}-\mathrm{Ind}\right), 3.48(\mathrm{dt}, 1 \mathrm{H}, J=4$ and $11.5 \mathrm{~Hz}, 6-\mathrm{H}), 3.90(\mathrm{~m}, 1 \mathrm{H}, 5-\mathrm{CH}), 6.06(\mathrm{~d}, 1 \mathrm{H}, J=9.5 \mathrm{~Hz}, N H \mathrm{Boc}), 6.58$ [m, 3H, $\left.\mathrm{NHC}\left(\mathrm{NH}_{2}\right)=\mathrm{N}\right], 6.90(\mathrm{~s}, 1 \mathrm{H}, 1-\mathrm{H}), 7.22$ (t, 1H, $\left.J=7.5 \mathrm{~Hz}, \mathrm{Ind}\right), 7.30$ (t, 1H, $J=7.5 \mathrm{~Hz}$, Ind), 7.51 (m, 1H, Ind), 7.61 (d, $1 \mathrm{H}, J=7.5 \mathrm{~Hz}$, Ind), 8.12 (d, $1 \mathrm{H}, J=7.5 \mathrm{~Hz}$, Ind). ${ }^{13} \mathrm{C}$ NMR (100 $\left.\mathrm{MHz},\left(\mathrm{CD}_{3}\right)_{2} \mathrm{CO}\right) \delta(\mathrm{ppm}): 12.5,18.2,19.5$ [3 $\left.\mathrm{CH}_{3}(\mathrm{Pbf})\right], 27.7$ [C $(\mathrm{Arg})$ and $\mathrm{CH}_{2}$-Ind], 28.3 [3 $\left.\mathrm{CH}_{3}(\mathrm{Boc})\right], 28.6\left[2 \mathrm{CH}_{3}(\mathrm{Pbf})\right], 28.7\left[3 \mathrm{CH}_{3}(\mathrm{Boc})\right], 29.6\left[\mathrm{C}_{\beta}(\mathrm{Arg})\right], 41.5\left[\mathrm{C}_{\delta}(\mathrm{Arg})\right], 43.6$ $\left[\mathrm{CH}_{2}(\mathrm{Pbf})\right], 46.1\left(\mathrm{C}_{6}\right), 51.8\left(\mathrm{C}_{5}\right), 53.6\left(\mathrm{C}_{5}-\mathrm{CH}\right), 56.7\left(\mathrm{C}_{3}\right), 79.4,83.9$ [2C (2Boc)], 86.9 [C (Pbf)], 115.6 [CH (Ind)], 117.4 [C (Pbf)], 118.9 [C (Ind)], 120.1, 123.2, 124.3, 124.9 [4CH (Ind)], 125.3 [C (Pbf)], 132.0 [C (Ind)], 132.8, 135.7 [2C (Pbf)], 136.4 [C (Ind)], 138.7 [C (Pbf)], 150.3, 156.8 [2CO (2Boc)], 157.3 [C $\left.\left(\mathrm{NHC}\left(\mathrm{NH}_{2}\right)=\mathrm{N}\right)\right], 158.9$ [C (Pbf)], $173.0\left(\mathrm{C}_{2}\right)$; ES-MS $m / z 810.9[\mathrm{M}+1]^{+}$; Anal. calcd. for $\mathrm{C}_{41} \mathrm{H}_{59} \mathrm{~N}_{7} \mathrm{O}_{8} \mathrm{~S}$ : C, 60.79; H, 7.34; N, 12.10. Found: C, 60.52; H, 7.51; N, 12.27.

\subsection{2. (5S,3S)-5-((S)-1-((tert-Butoxycarbonyl)amino-2-(1-(tert-butoxycarbonyl)-indol-3-yl)} ethyl-3-(3-(2-((2,2,4,6,7-pentamethyl-2,3-dihydrobenzofuran-5-yl)sulfonyl)guanidino)propyl)-2-oxopiperazine [(S)-6a]

White solid (467 mg, 20\%); $[\alpha]_{\mathrm{D}}{ }^{20}-15\left(c\right.$ 1.1, $\left.\mathrm{CH}_{2} \mathrm{Cl}_{2}\right)$; $\mathrm{Mp}: 118-120{ }^{\circ} \mathrm{C}$ (EtOAc/hexane); HPLC [Sunfire $\mathrm{C}_{18}(4.6 \times 150 \mathrm{~mm}, 3.5 \mu \mathrm{m})$, isocratic $50 \%$ of solvent $\mathrm{A}$ ] $t_{\mathrm{R}} 9.05 \mathrm{~min} ;{ }^{1} \mathrm{H}$

\footnotetext{
${ }^{1}$ As the 2-oxopiperazine ring is the common structure of the compounds, we have preferred to give preference to this ring in the nomenclature of the compounds with respect to other senior groups or skeletons in the IUPAC nomenclature system.
} 
NMR (400 MHz, $\left.\left(\mathrm{CD}_{3}\right)_{2} \mathrm{CO}\right) \delta(\mathrm{ppm}): 1.33$ (s, 9H, Boc), 1.42 [s, 6H, 2CH $\left.3(\mathrm{Pbf})\right], 1.66$ [s, 3H, Boc (Ind)], 1.68 [m, 2H, $\gamma-\mathrm{H}$ (Arg)], 1.70 [m, 1H, $\beta$-H (Arg)], 1.92 [m, 1H, $\beta$-H (Arg)], $2.05\left[\mathrm{~s}, 3 \mathrm{H}, \mathrm{CH}_{3}(\mathrm{Pbf})\right], 2.51$ [s, 3H, $\left.\mathrm{CH}_{3}(\mathrm{Pbf})\right], 2.59$ [s, 3H, $\left.\mathrm{CH}_{3}(\mathrm{Pbf})\right], 2.96$ (m, 1H, $\mathrm{CH}_{2^{-}}$ Ind), 2.97 [s, 2H, $\mathrm{CH}_{2}$ (Pbf)], 3.07 (m, 1H, $\mathrm{CH}_{2}$-Ind), 3.12 (m, 1H, 5-H), 3.24 [m, 2H, $\delta-\mathrm{H}$ (Arg)], 3.27 (m, 2H, 6-H), 3.32 [m, 1H, 3-H], 4.00 (m, 1H, 5-CH), 6.09 (d, 1H, J = 9.5 Hz, NHBoc), 6.53 [m, 3H, NHC( $\left.\left.\mathrm{NH}_{2}\right)=\mathrm{N}\right], 6.85(\mathrm{~s}, 1 \mathrm{H}, 1-\mathrm{H}), 7.23$ (t, 1H, J = 7.5 Hz, Ind), 7.31 (t, $1 \mathrm{H}, J=7.5 \mathrm{~Hz}$, Ind), 7.54 (m, 1H, Ind), 7.68 (d, 1H, $J=7.5 \mathrm{~Hz}$, Ind), 8.12 (d, 1H, $J=7.5 \mathrm{~Hz}$, Ind). ${ }^{13} \mathrm{C}$ NMR (100 MHz, $\left.\left(\mathrm{CD}_{3}\right)_{2} \mathrm{CO}\right) \delta$ (ppm): 12.6, 18.2, $19.5\left[3 \mathrm{CH}_{3}(\mathrm{Pbf})\right], 26.5\left[\mathrm{C}_{\gamma}(\mathrm{Arg})\right.$

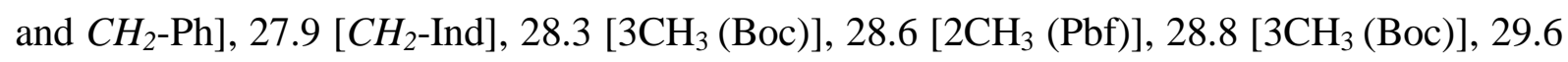
$\left[\mathrm{C}_{\beta}(\mathrm{Arg})\right], 41.6\left[\mathrm{C}_{\delta}(\mathrm{Arg})\right], 43.6\left[\mathrm{CH}_{2}(\mathrm{Pbf})\right], 46.1\left(\mathrm{C}_{6}\right), 53.0\left(\mathrm{C}_{5}-\mathrm{CH}\right), 55.7\left(\mathrm{C}_{5}\right), 59.2\left(\mathrm{C}_{3}\right)$, 78.9, 84.0 [2C (2Boc)], 86.9 [C (Pbf)], 115.8 [CH (Ind)], 117.4 [C (Pbf)], 118.5 [C (Ind)], 120.1, 123.2, 124.5, 125.0 [4CH (Ind)], 125.4 [C (Pbf)], 131.8 [C (Ind)], 132.8, 135.6 [2C (Pbf)], $136.4 \quad[\mathrm{C} \quad(\mathrm{Ind})], \quad 138.7 \quad[\mathrm{C} \quad(\mathrm{Pbf})], \quad 150.3,156.8 \quad[2 \mathrm{CO} \quad$ (2Boc) $], \quad 157.3 \quad[\mathrm{C}$ $\left.\left(\mathrm{NHC}\left(\mathrm{NH}_{2}\right)=\mathrm{N}\right)\right], 158.9[\mathrm{C}(\mathrm{Pbf})], 171.8\left(\mathrm{C}_{2}\right]$; ES-MS m/z $810.9[\mathrm{M}+1]^{+}$; Anal. calcd. for $\mathrm{C}_{41} \mathrm{H}_{59} \mathrm{~N}_{7} \mathrm{O}_{8} \mathrm{~S}$ : C, 60.79; H, 7.34; N, 12.10. Found: C, 60.97; H, 7.15; N, 12.32 .

\subsection{3. (5R,3S)-5-((S)-1-((tert-Butoxycarbonyl)amino-2-(1-(tert-butoxycarbonyl)-indol-3-yl)- ethyl-3-(4-((tert-butoxycarbonyl)amino)butyl)-2-oxopiperazine [(R)-6b]}

White solid (726 mg, 40\%); $[\alpha]_{\mathrm{D}}{ }^{20}-12$ (c 1.6, $\mathrm{CH}_{2} \mathrm{Cl}_{2}$ ); $\mathrm{Mp}$ : 84-86 ${ }^{\circ} \mathrm{C}$ (EtOAc/hexane); HPLC [Sunfire $\mathrm{C}_{18}(4.6 \times 150 \mathrm{~mm}, 3.5 \mu \mathrm{m}), 30-100 \%$ gradient of solvent A in B, $30 \mathrm{~min}$ ] $t_{\mathrm{R}}$ $13.89 \mathrm{~min} ;{ }^{1} \mathrm{H}$ NMR $\left(400 \mathrm{MHz},\left(\mathrm{CD}_{3}\right)_{2} \mathrm{CO}\right) \delta(\mathrm{ppm}): 1.31$ (s, 9H, Boc), 1.37 [s, 9H, Boc (Lys)], 1.52 [m, 2H, $\gamma-\mathrm{H}$ (Lys)], 1.54 [m, 2H, $\delta$-H (Lys)], 1.66 [s, 9H, Boc (Ind)], 1.69 [m, 1H, $\beta$-H (Lys)], 1.84 [m, 1H, $\beta$-H (Lys)], 2.40 (m, 1H, 4-H), 2.89 (m, 1H, $C_{2}$-Ind), 3.10 [m, 2H, $\varepsilon-\mathrm{H}$ (Lys)], 3.17 (m, 1H, 5-H), 3.26 (m, 1H, 6-H), 3.31 (m, 1H, 3-H), 3.43 (m, 1H, $\mathrm{CH}_{2^{-}}$ Ind), $3.47(\mathrm{~m}, 1 \mathrm{H}, 6-\mathrm{H}), 3.87$ (ddd, $1 \mathrm{H}, J=3,9.5$ and $18.5 \mathrm{~Hz}, 5-\mathrm{CH}), 5.95[\mathrm{~m}, 1 \mathrm{H}$, NHBoc (Lys)], 6.01 (d, 1H, $J=9.5 \mathrm{~Hz}, N H B o c), 6.78$ (s, 1H, 1-H), 7.23 (t, 1H, $J=7.5 \mathrm{~Hz}$, Ind), 7.30 (t, $1 \mathrm{H}, J=7.5 \mathrm{~Hz}$, Ind), 7.51 (m, 1H, Ind), 7.63 (d, 1H, $J=7.5 \mathrm{~Hz}$, Ind), 8.12 (d, 1H, $J=7.5$ $\mathrm{Hz}$, Ind). ${ }^{13} \mathrm{C}$ NMR $\left(100 \mathrm{MHz},\left(\mathrm{CD}_{3}\right)_{2} \mathrm{CO}\right) \delta(\mathrm{ppm}): 24.6\left[\mathrm{C}_{\gamma}\right.$ (Lys)], 27.7 [ $\mathrm{CH}_{2}$-Ind], 28.3, 28.6, $28.7\left[9 \mathrm{CH}_{3}(3 \mathrm{Boc})\right], 30.7\left[\mathrm{C}_{\delta}(\mathrm{Lys})\right], 31.2\left[\mathrm{C}_{\beta}(\mathrm{Lys})\right], 41.2\left[\mathrm{C}_{\varepsilon}(\mathrm{Lys})\right], 46.4\left(\mathrm{C}_{6}\right), 52.0$ $\left(\mathrm{C}_{5}\right), 53.6\left(\mathrm{C}_{5}-\mathrm{CH}\right), 57.7\left(\mathrm{C}_{3}\right), 78.3,78.7,83.9$ [3C (3Boc)], 115.8 [CH (Ind)], 118.9 [C (Ind)], 120.1, 123.1, 124.3, 124.9 [4CH (Ind)], 132.1, 136.3 [2C (Ind)], 150.3, 156.6 [3CO (3Boc)], $173.0\left(\mathrm{C}_{2}\right)$; ES-MS m/z $630.8[\mathrm{M}+1]^{+}$; Anal. calcd. for $\mathrm{C}_{33} \mathrm{H}_{51} \mathrm{~N}_{5} \mathrm{O}_{7}: \mathrm{C}, 62.93 ; \mathrm{H}, 8.16 ; \mathrm{N}$, 11.12. Found: C, 62.66; H, 8.37; N, 10.95 . 
4.3.4. (5S,3S)-5-((S)-1-((tert-Butoxycarbonyl)amino-2-(1-(tert-butoxycarbonyl)-indol-3-yl)ethyl-3-(4-((tert-butoxycarbonyl)amino)butyl)-2-oxopiperazine [(S)-6b]

White solid (363 mg, 20\%); $[\alpha]_{\mathrm{D}}{ }^{20}-10\left(c\right.$ 1.4, $\left.\mathrm{CH}_{2} \mathrm{Cl}_{2}\right)$; Mp: $76-78{ }^{\circ} \mathrm{C}$ (EtOAc/hexane); HPLC [Sunfire $\mathrm{C}_{18}(4.6 \times 150 \mathrm{~mm}, 3.5 \mu \mathrm{m}), 30-100 \%$ gradient of solvent $\mathrm{A}$ in $30 \mathrm{~min}$ ] $t_{\mathrm{R}}$ $13.56 \mathrm{~min} ;{ }^{1} \mathrm{H}$ NMR $\left(400 \mathrm{MHz},\left(\mathrm{CD}_{3}\right)_{2} \mathrm{CO}\right) \delta(\mathrm{ppm}): 1.35$ (s, 9H, Boc), 1.39 [s, 9H, Boc (Lys)], 1.48 [m, 2H, $\gamma-\mathrm{H}$ (Lys)], 1.50 [m, 2H, $\delta$-H (Lys)], 1.59 [m, 1H, $\beta$-H (Lys)], 1.67 [s, 9H, Boc (Ind)], 1.94 [m, 1H, $\beta$-H (Lys)], 2.99 (dd, $1 \mathrm{H}, J=8$ and $14.5 \mathrm{~Hz}, \mathrm{CH}_{2}$-Ind), 3.08 [m, 2H, ع-H (Lys)], 3.10 (m, 1H, $\mathrm{CH}_{2}$-Ind), 3.12 (m, 1H, 5-H), 3.26 (m, 2H, 6-H), 3.27 (m, 1H, 3H), $4.00(\mathrm{~m}, 1 \mathrm{H}, 5-\mathrm{CH}), 5.94$ [m, 1H, NHBoc (Lys)], 6.06 (d, 1H, J = 9 Hz, NHBoc), 6.73 (s, $1 \mathrm{H}, 1-\mathrm{H}), 7.25$ (t, 1H, $J=7.5 \mathrm{~Hz}$, Ind), 7.31 (t, 1H, $J=7.5 \mathrm{~Hz}$, Ind), 7.55 (m, 1H, Ind), 7.70 (d, $1 \mathrm{H}, J=7.5 \mathrm{~Hz}$, Ind), 8.13 (d, $1 \mathrm{H}, J=7.5 \mathrm{~Hz}$, Ind). ${ }^{13} \mathrm{C} \mathrm{NMR}\left(100 \mathrm{MHz},\left(\mathrm{CD}_{3}\right)_{2} \mathrm{CO}\right) \delta$

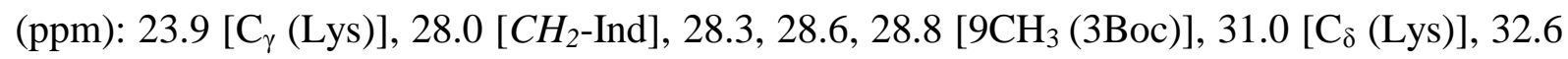
[C $\mathrm{C}_{\beta}$ (Lys)], $41.1\left[\mathrm{C}_{\varepsilon}(\mathrm{Lys})\right], 46.2\left(\mathrm{C}_{6}\right), 53.0\left(\mathrm{C}_{5}-\mathrm{CH}\right), 55.5\left(\mathrm{C}_{5}\right), 59.7\left(\mathrm{C}_{3}\right), 78.3,78.9,84.0[3 \mathrm{C}$ (3Boc)], 115.6 [CH (Ind)], 118.5 [C (Ind)], 120.1, 123.3, 124.6, 125.0 [4CH (Ind)], 131.8, 136.4 [2C (Ind)], 150.3, 156.7 [3CO (3Boc)], $171.7\left(\mathrm{C}_{2}\right)$; ES-MS m/z 630.8 [M+1] ${ }^{+}$; Anal. calcd. for $\mathrm{C}_{33} \mathrm{H}_{51} \mathrm{~N}_{5} \mathrm{O}_{7}$ : C, 62.93; H, 8.16; N, 11.12. Found: C, 62.83; H, 8.02; N, 10.90 .

\subsection{5. (5R,3S)-5-((S)-1-(tert-Butoxycarbonyl)amino-2-phenylethyl)-3-(3-(2-((2,2,4,6,7-}

pentamethyl-2,3-dihydrobenzofuran-5-yl)sulfonyl)guanidino) propyl)-2-oxopiperazine [(R)-7a]

White solid (754 mg, 39\%); $[\alpha]_{\mathrm{D}}{ }^{20}-6\left(c\right.$ 1.0, $\left.\mathrm{CH}_{2} \mathrm{Cl}_{2}\right)$; Mp: 136-138 ${ }^{\circ} \mathrm{C}$ (EtOAc/hexane); HPLC [Sunfire $\mathrm{C}_{18}(4.6 \times 150 \mathrm{~mm}, 3.5 \mu \mathrm{m}), 10-100 \%$ gradient of solvent A in B, $30 \mathrm{~min}$ ] $t_{\mathrm{R}}$ $18.05 \mathrm{~min} ;{ }^{1} \mathrm{H}$ NMR (400 MHz, $\left.\left(\mathrm{CD}_{3}\right)_{2} \mathrm{CO}\right) \delta(\mathrm{ppm}): 1.31$ (s, 9H, Boc), $1.44\left[\mathrm{~s}, 6 \mathrm{H}, 2 \mathrm{CH}_{3}\right.$ (Pbf)], 1.60 [m, 2H, $\gamma-\mathrm{H}(\mathrm{Arg})], 1.70$ [m, 1H, $\beta-\mathrm{H}$ (Arg)], 1.80 [m, 1H, $\beta-\mathrm{H}(\mathrm{Arg})], 2.06$ [s, $\left.3 \mathrm{H}, \mathrm{CH}_{3}(\mathrm{Pbf})\right], 2.35$ (m, 1H, 4-H), 2.51 [s, 3H, $\left.\mathrm{CH}_{3}(\mathrm{Pbf})\right], 2.59$ [s, 3H, $\left.\mathrm{CH}_{3}(\mathrm{Pbf})\right], 2.75$ (dd, $1 \mathrm{H}, J=10$ and $\left.14 \mathrm{~Hz}, \mathrm{CH}_{2}-\mathrm{Ph}\right), 2.98\left[\mathrm{~s}, 2 \mathrm{H}, \mathrm{CH}_{2}(\mathrm{Pbf})\right], 3.07(\mathrm{~m}, 1 \mathrm{H}, 5-\mathrm{H}), 3.18[\mathrm{~m}, 1 \mathrm{H}, \delta-\mathrm{H}$ (Arg)], $3.23\left(\mathrm{~m}, 1 \mathrm{H}, \mathrm{CH}_{2}-\mathrm{Ph}\right), 3.24(\mathrm{~m}, 1 \mathrm{H}, 6-\mathrm{H}), 3.30$ [m, 2H, $\delta-\mathrm{H}(\mathrm{Arg})$ and 3-H], 3.43 (dt, $1 \mathrm{H}, J=4$ and $12 \mathrm{~Hz}, 6-\mathrm{H}), 3.83(\mathrm{ddd}, 1 \mathrm{H}, J=4,9.5$ and $18 \mathrm{~Hz}, 5-\mathrm{CH}), 5.96(\mathrm{~d}, 1 \mathrm{H}, J=9.5$ $\mathrm{Hz}, N H \mathrm{Boc}), 6.58$ [m, 3H, NHC( $\left.\left.\mathrm{NH}_{2}\right)=\mathrm{N}\right], 6.85(\mathrm{~s}, 1 \mathrm{H}, 1-\mathrm{H}), 7.16(\mathrm{~m}, 1 \mathrm{H}, \mathrm{Ph}), 7.20-7.28(\mathrm{~m}$, $4 \mathrm{H}, \mathrm{Ph}) .{ }^{13} \mathrm{C}$ NMR $\left(100 \mathrm{MHz},\left(\mathrm{CD}_{3}\right)_{2} \mathrm{CO}\right) \delta(\mathrm{ppm}): 13.2,18.9,20.2\left[3 \mathrm{CH}_{3}(\mathrm{Pbf})\right], 28.0\left[\mathrm{C}_{\gamma}\right.$ (Arg), $29.2\left[3 \mathrm{CH}_{3}\right.$ (Boc)], $29.4\left[2 \mathrm{CH}_{3}\right.$ (Pbf)], $29.5\left[\mathrm{C}_{\beta}(\mathrm{Arg})\right], 38.8\left[\mathrm{CH}_{2} \mathrm{Ph}\right], 42.2\left[\mathrm{C}_{\delta}\right.$ (Arg) $], 44.3\left[\mathrm{CH}_{2}(\mathrm{Pbf})\right], 46.6\left(\mathrm{C}_{6}\right), 52.4\left(\mathrm{C}_{5}\right), 55.8\left(\mathrm{C}_{5^{-}} \mathrm{CH}\right), 57.4\left(\mathrm{C}_{3}\right), 79.4$ [C (Boc) ], 87.6, 
118.1 [2C (Pbf)], 125.9 [C (Pbf)], 127.4, 129.6, 130.9 [5CH (Ph)], 133.5, 136.6, 139.4 [3C $(\mathrm{Pbf})], 140.9$ [C $(\mathrm{Ph})], 158.0[\mathrm{CO}(\mathrm{Boc})], 157.3\left[\mathrm{C}\left(\mathrm{NHC}\left(\mathrm{NH}_{2}\right)=\mathrm{N}\right)\right], 159.5[\mathrm{C}(\mathrm{Pbf})], 173.6$ $\left[\mathrm{C}_{2}\right]$; ES-MS $m / z$, $671.6[\mathrm{M}+1]^{+}$; Anal. calcd. for $\mathrm{C}_{34} \mathrm{H}_{50} \mathrm{~N}_{6} \mathrm{O}_{6} \mathrm{~S}: \mathrm{C}, 60.87 ; \mathrm{H}, 7.51 ; \mathrm{N}, 12.53$. Found: C, 60.98; H; 7.73; N, 12.29.

\subsection{6. (5S,3S)-5-((S)-1-(tert-Butoxycarbonyl)amino-2-phenylethyl)-3-(3-(2-((2,2,4,6,7-} pentamethyl-2,3-dihydrobenzofuran-5-yl)sulfonyl)guanidino) propyl)-2-oxopiperazine $[(\mathbf{S})-7 a]$

White solid (792 mg, 41\%); $[\alpha]_{\mathrm{D}}{ }^{20}-27\left(c\right.$ 1.0, $\left.\mathrm{CH}_{2} \mathrm{Cl}_{2}\right)$; Mp: $138-140{ }^{\circ} \mathrm{C}$ (EtOAc/hexane); HPLC [Sunfire $\mathrm{C}_{18}(4.6 \times 150 \mathrm{~mm}, 3.5 \mu \mathrm{m}), 10-100 \%$ gradient of solvent $\mathrm{A}$ in B,30 min] $t_{\mathrm{R}}$ $18.05 \mathrm{~min} ;{ }^{1} \mathrm{H}$ NMR (400 MHz, $\left.\left(\mathrm{CD}_{3}\right)_{2} \mathrm{CO}\right) \delta(\mathrm{ppm}): 1.31$ (s, 9H, Boc), 1.44 [s, 6H, 2CH (Pbf)], 1.60 [m, 2H, $\gamma-\mathrm{H}(\mathrm{Arg})], 1.80$ [m, 1H, $\beta-\mathrm{H}$ (Arg)], 1.90 [m, 1H, $\beta-\mathrm{H}(\mathrm{Arg})], 2.06$ [s, $\left.3 \mathrm{H}, \mathrm{CH}_{3}(\mathrm{Pbf})\right], 2.51$ [s, 3H, $\left.\mathrm{CH}_{3}(\mathrm{Pbf})\right], 2.59$ [s, 3H, $\left.\mathrm{CH}_{3}(\mathrm{Pbf})\right], 2.82$ (m, 1H, $\left.\mathrm{CH}_{2}-\mathrm{Ph}\right), 2.96$ (m, 1H, $\mathrm{CH}_{2}-\mathrm{Ph}$ ), 3.00 [s, 2H, $\left.\mathrm{CH}_{2}(\mathrm{Pbf})\right], 3.02(\mathrm{~m}, 1 \mathrm{H}, 5-\mathrm{H}), 3.22$ [m, 2H, $\delta-\mathrm{H}$ (Arg)], 3.24 (m, 2H, 6-H), $3.28[\mathrm{~m}, 1 \mathrm{H}, 3-\mathrm{H}], 3.87(\mathrm{~m}, 1 \mathrm{H}, 5-\mathrm{CH}), 5.98$ (d, 1H, J = $9 \mathrm{~Hz}, N H \mathrm{Boc}), 6.52$ $\left[\mathrm{m}, 3 \mathrm{H}, \mathrm{NHC}\left(\mathrm{NH}_{2}\right)=\mathrm{N}\right], 6.82(\mathrm{~s}, 1 \mathrm{H}, 1-\mathrm{H}), 7.18(\mathrm{~m}, 1 \mathrm{H}, \mathrm{Ph}), 7.23-7.33(\mathrm{~m}, 4 \mathrm{H}, \mathrm{Ph}) .{ }^{13} \mathrm{C} \mathrm{NMR}$ $\left(100 \mathrm{MHz},\left(\mathrm{CD}_{3}\right)_{2} \mathrm{CO}\right) \delta(\mathrm{ppm}): 13.2,18.9,20.2\left[3 \mathrm{CH}_{3}(\mathrm{Pbf})\right], 27.1\left[\mathrm{C}_{\gamma}(\mathrm{Arg})\right], 29.2\left[2 \mathrm{CH}_{3}\right.$ (Pbf)], $29.4\left[3 \mathrm{CH}_{3}\right.$ (Boc)], $29.6\left[\mathrm{C}_{\beta}(\mathrm{Arg})\right], 39.1\left[\mathrm{CH}_{2}-\mathrm{Ph}\right], 41.2\left[\mathrm{C}_{\delta}(\mathrm{Arg})\right], 44.3\left[\mathrm{CH}_{2}(\mathrm{Pbf})\right]$, $46.8\left(\mathrm{C}_{6}\right), 55.2\left[\mathrm{C}_{5^{-}} \mathrm{CH}\right], 56.2\left(\mathrm{C}_{5}\right), 59.9\left(\mathrm{C}_{3}\right), 79.5$ [C (Boc)], 87.6, 118.1 [2C (Pbf)], 125.9 [C (Pbf)], 127.6, 129.7, 130.8 [5CH (Ph)], 133.5, 136.6, 139.4 [3C (Pbf)], 140.5 [C (Ph)], 157.3 [CO (Boc)], 158.0 [C $\left.\left(\mathrm{NHC}\left(\mathrm{NH}_{2}\right)=\mathrm{N}\right)\right], 159.6$ [C (Pbf)], $172.5\left(\mathrm{C}_{2}\right)$; ES-MS m/z 671.2 $[\mathrm{M}+1]^{+}$; Anal. calcd. for $\mathrm{C}_{34} \mathrm{H}_{50} \mathrm{~N}_{6} \mathrm{O}_{6} \mathrm{~S}: \mathrm{C}, 60.87 ; \mathrm{H}, 7.51 ; \mathrm{N}, 12.53$. Found: $\mathrm{C}, 60.69 ; \mathrm{H}, 7.69$; $\mathrm{N}, 12.68$.

4.4. General procedure for the synthesis of the 3,6-dioxooctahydroimidazo[1,5-a]pyrazines (R)- and (S)-8a and -9a

The corresponding 2-oxopiperazine (R)- and (S)-6a and -7a $(0.31 \mathrm{mmol})$ was dissolved in $3.4 \mathrm{~N}$ solution of $\mathrm{HCl}$ in EtOAc $(5 \mathrm{~mL})$ and the mixture was stirred at room temperature for $30 \mathrm{~min}$. Afterward, the solvent was evaporated to dryness, the residue was dissolved in $\mathrm{CH}_{3} \mathrm{CN} / \mathrm{H}_{2} \mathrm{O}(1: 3,2 \mathrm{~mL})$ and the solution was lyophilized. Then, TEA (87 $\left.\mu \mathrm{L}, 0.62 \mathrm{mmol}\right)$ was added to a solution of the lyophilized powder in $\mathrm{CH}_{2} \mathrm{Cl}_{2}(10 \mathrm{~mL})$. After $15 \mathrm{~min}$ of stirring at $0{ }^{\circ} \mathrm{C}$, TEA $(101 \mu \mathrm{L}, 0.73 \mathrm{mmol})$ and triphosgene $(36 \mathrm{mg}, 0.12 \mathrm{mmol})$ were successively added. The mixture was stirred at $0{ }^{\circ} \mathrm{C}$ for $2 \mathrm{~h}$ and the reaction was diluted $\mathrm{CH}_{2} \mathrm{Cl}_{2}(10 \mathrm{~mL})$. 
The solution was successively washed with $\mathrm{H}_{2} \mathrm{O}(2 \times 5 \mathrm{~mL})$ and brine $(5 \mathrm{~mL})$, dried over $\mathrm{Na}_{2} \mathrm{SO}_{4}$ and evaporated to dryness. The residue was purified by flash chromatography, using $\mathrm{MeOH}$ in $\mathrm{CH}_{2} \mathrm{Cl}_{2}$ gradient as mobile phase to obtain the respective 3,6dioxooctahydroimidazo[1,5-a]pyrazine (R)- and (S)-8a and -9a.

\subsection{1. (1S,5S,8aR)-1-(Indol-3-yl)methyl-5-(3-(2-((2,2,4,6,7-pentamethyl-2,3-dihydro-}

benzofuran-5-yl)sulfonyl)guanidino) propyl)-3,6-dioxooctahydroimidazo[1,5-a]pyrazine [(R)-8a]

Foam (118 mg, 60\%); $[\alpha]_{\mathrm{D}}{ }^{20}-4.4\left(c 0.8, \mathrm{CH}_{2} \mathrm{Cl}_{2}\right)$; HPLC [Sunfire $\mathrm{C}_{18}(4.6 \times 150 \mathrm{~mm}, 3.5$ $\mu \mathrm{m}), 10-60 \%$ gradient of solvent $\mathrm{A}$ in $\mathrm{B}, 30 \mathrm{~min}] t_{\mathrm{R}} 27.20 \mathrm{~min} ;{ }^{1} \mathrm{H}$ NMR (400 MHz, $\left.\left(\mathrm{CD}_{3}\right)_{2} \mathrm{CO}\right) \delta(\mathrm{ppm}): 1.42\left[\mathrm{~s}, 6 \mathrm{H}, 2 \mathrm{CH}_{3}(\mathrm{Pbf})\right], 1.58[\mathrm{~m}, 2 \mathrm{H}, \gamma-\mathrm{H}(\mathrm{Arg})], 1.70[\mathrm{~m}, 1 \mathrm{H}, \beta-\mathrm{H}$ (Arg)], 1.90 [m, 1H, $\beta-\mathrm{H}$ (Arg)], 2.03 [s, 3H, $\mathrm{CH}_{3}$ (Pbf)], 2.48 [s, 3H, $\left.\mathrm{CH}_{3}(\mathrm{Pbf})\right], 2.56$ [s, 3H, $\mathrm{CH}_{3}(\mathrm{Pbf})$ ], 2.97 [s, 2H, CH 2 (Pbf)], 3.04 (d, 2H, $\left.J=8.5 \mathrm{~Hz}, 1-\mathrm{CH}_{2}\right), 3.21$ [m, 2H, $\delta-\mathrm{H}$ (Arg)], $3.30(\mathrm{dt}, 1 \mathrm{H}, J=4.5$ and $11.5 \mathrm{~Hz}, 8-\mathrm{H}), 3.68(\mathrm{t}, 1 \mathrm{H}, J=11.5 \mathrm{~Hz}, 8-\mathrm{H}), 4.00$ (ddd, $1 \mathrm{H}, J=4.5$, 7.5 and $11.5 \mathrm{~Hz}, 8 \mathrm{a}-\mathrm{H}), 4.14(\mathrm{dd}, 1 \mathrm{H}, J=4$ and $9.5 \mathrm{~Hz}, 5-\mathrm{H}), 4.41$ (q, 1H, $J=7.5 \mathrm{~Hz}, 1-\mathrm{H})$, $5.94(\mathrm{bs}, 1 \mathrm{H}, 2-\mathrm{H}), 6.50\left[\mathrm{~m}, 3 \mathrm{H}, \mathrm{NHC}\left(\mathrm{NH}_{2}\right)=\mathrm{N}\right], 7.03(\mathrm{dd}, 1 \mathrm{H}, J=4$ and $11 \mathrm{~Hz}, \mathrm{Ind}), 7.10(\mathrm{~m}$, 2H, 1-H and Ind), 7.31 (d, 1H, $J=2.5 \mathrm{~Hz}$, Ind), 7.41 (d, 1H, $J=8 \mathrm{~Hz}$, Ind), 7.61 (d, 1H, $J=$ $7.5 \mathrm{~Hz}, \mathrm{Ind}), 10.18$ [m, 1H, NH (Ind)]; ${ }^{13} \mathrm{C} \mathrm{NMR}\left(100 \mathrm{MHz},\left(\mathrm{CD}_{3}\right)_{2} \mathrm{CO}\right) \delta$ (ppm): 12.5, 18.2, $19.5\left[3 \mathrm{CH}_{3}(\mathrm{Pbf})\right], 25.7\left[1-\mathrm{CH}_{2}\right], 26.3\left[\mathrm{C}_{\gamma}(\mathrm{Arg})\right], 28.7\left[2 \mathrm{CH}_{3}(\mathrm{Pbf})\right], 30.5\left[\mathrm{C}_{\beta}(\mathrm{Arg})\right], 40.6$ $\left(\mathrm{C}_{8}\right), 41.2\left[\mathrm{C}_{\delta}(\mathrm{Arg})\right], 43.6\left[\mathrm{CH}_{2}(\mathrm{Pbf})\right], 52.1\left(\mathrm{C}_{8 \mathrm{a}}\right), 53.7\left(\mathrm{C}_{5}\right.$ and $\left.\mathrm{C}_{1}\right), 86.9[\mathrm{C}(\mathrm{Pbf})], 111.5[\mathrm{C}$ (Ind)], 112.3 [CH (Ind)], 117.4 [C (Pbf)], 119.2, 119.6, 122.4, 123.8 [4CH (Ind)], 125.3 [C (Pbf)], 128.2 [C (Ind)], 132.8, 135.8 [2C (Pbf)], 137.7 [C (Ind)], 138.7 [C (Pbf)], 157.3 [C $\left.\left(\mathrm{NHC}\left(\mathrm{NH}_{2}\right)=\mathrm{N}\right)\right], 158.8[\mathrm{C}(\mathrm{Pbf})], 161.8\left(\mathrm{C}_{3}\right), 170.0\left(\mathrm{C}_{6}\right)$; ES-MS m/z $636.6[\mathrm{M}+1]^{+}$; Anal. calcd. for $\mathrm{C}_{32} \mathrm{H}_{41} \mathrm{~N}_{7} \mathrm{O}_{5} \mathrm{~S}: \mathrm{C}, 60.45 ; \mathrm{H}, 6.50 ; \mathrm{N}, 15.42$. Found: C, 60.64; H, 6.75; N, 15.20.

\subsection{2. (1S,5S,8aS)-1-(Indol-3-yl)methyl-5-(3-(2-((2,2,4,6,7-pentamethyl-2,3-dihydro-}

benzofuran-5-yl)sulfonyl)guanidino) propyl)-3,6-dioxooctahydroimidazo[1,5-a]pyrazine $[(\mathbf{S})-8 a]$

Foam (118 mg, 60\%); $[\alpha]_{\mathrm{D}}{ }^{20}-4.6\left(c 0.6, \mathrm{CH}_{2} \mathrm{Cl}_{2}\right)$; HPLC [Sunfire $\mathrm{C}_{18}(4.6 \times 150 \mathrm{~mm}, 3.5$ $\mu \mathrm{m}), 10-60 \%$ gradient of solvent $\mathrm{A}$ in $\mathrm{B}, 30 \mathrm{~min}] t_{\mathrm{R}} 27.39 \mathrm{~min} ;{ }^{1} \mathrm{H}$ NMR (400 MHz, $\left.\left(\mathrm{CD}_{3}\right)_{2} \mathrm{CO}\right) \delta(\mathrm{ppm}): 1.42\left[\mathrm{~s}, 6 \mathrm{H}, 2 \mathrm{CH}_{3}(\mathrm{Pbf})\right], 1.40[\mathrm{~m}, 2 \mathrm{H}, \gamma-\mathrm{H}(\mathrm{Arg})], 1.65[\mathrm{~m}, 1 \mathrm{H}, \beta-\mathrm{H}$ (Arg)], 1.96 [m, 1H, $\beta-\mathrm{H}(\mathrm{Arg})], 2.04$ [s, 3H, $\mathrm{CH}_{3}$ (Pbf)], 2.42 [ddd, 1H, J= 5, 10.5 and 18.5 
$\mathrm{Hz}, \delta-\mathrm{H}(\mathrm{Arg})], 2.48$ [s, 3H, $\left.\mathrm{CH}_{3}(\mathrm{Pbf})\right], 2.56$ [s, 3H, $\left.\mathrm{CH}_{3}(\mathrm{Pbf})\right], 2.78$ (ddd, 1H, J = 3.5, 5.5 and $11.5 \mathrm{~Hz}, 8-\mathrm{H}), 2.97$ [s, 2H, $\left.\mathrm{CH}_{2}(\mathrm{Pbf})\right], 3.02\left(\mathrm{~m}, 1 \mathrm{H}, 1-\mathrm{CH}_{2}\right), 3.06(\mathrm{~m}, 1 \mathrm{H}, 8-\mathrm{H}), 3.16[\mathrm{~m}$, $1 \mathrm{H}, \delta-\mathrm{H}(\mathrm{Arg})], 3.17\left(\mathrm{~m}, 1 \mathrm{H}, 1-\mathrm{CH}_{2}\right), 3.52(\mathrm{dt}, 1 \mathrm{H}, J=3.5$ and $10 \mathrm{~Hz}, 8 \mathrm{a}-\mathrm{H}), 3.85(\mathrm{~m}, 1 \mathrm{H}, 1-$ H), $3.86(\mathrm{~m}, 1 \mathrm{H}, 5-\mathrm{H}), 6.00(\mathrm{~m}, 1 \mathrm{H}, 2-\mathrm{H}), 6.27\left[\mathrm{~m}, 1 \mathrm{H}, \mathrm{NHC}\left(\mathrm{NH}_{2}\right)=\mathrm{N}\right], 6.50[\mathrm{~m}, 2 \mathrm{H}$, $\left.\mathrm{NHC}\left(\mathrm{NH}_{2}\right)=\mathrm{N}\right], 7.02(\mathrm{ddd}, 1 \mathrm{H}, J=1,7$ and $8 \mathrm{~Hz}, \mathrm{Ind}), 7.03(\mathrm{~m}, 1 \mathrm{H}, 1-\mathrm{H}), 7.11(\mathrm{ddd}, 1 \mathrm{H}, J=$ 1, 7 and $8 \mathrm{~Hz}$, Ind), 7.29 (d, 1H, $J=2.5 \mathrm{~Hz}$, Ind), 7.40 (dt, 1H, $J=1$ and $8 \mathrm{~Hz}$, Ind), 7.61 (dd, $1 \mathrm{H}, J=1$ and $8 \mathrm{~Hz}, \mathrm{Ind}), 10.18$ [m, $1 \mathrm{H}, \mathrm{NH}(\mathrm{Ind})] ;{ }^{13} \mathrm{C} \mathrm{NMR}\left(100 \mathrm{MHz},\left(\mathrm{CD}_{3}\right)_{2} \mathrm{CO}\right) \delta(\mathrm{ppm})$ : 12.5, 18.2, $19.5\left[3 \mathrm{CH}_{3}(\mathrm{Pbf})\right], 24.8\left[\mathrm{C}_{\gamma}(\mathrm{Arg})\right], 28.3\left[\mathrm{C}_{\beta}(\mathrm{Arg})\right], 28.7\left[2 \mathrm{CH}_{3}(\mathrm{Pbf})\right], 30.7$ [1$\left.\mathrm{CH}_{2}\right], 41.4\left[\mathrm{C}_{\delta}(\mathrm{Arg})\right], 43.6\left[\mathrm{CH}_{2}(\mathrm{Pbf})\right], 44.9\left(\mathrm{C}_{8}\right), 55.9\left(\mathrm{C}_{8 \mathrm{a}}\right), 57.9\left(\mathrm{C}_{5}\right), 59.7\left(\mathrm{C}_{1}\right), 86.9[\mathrm{C}$ (Pbf)], 110.9 [C (Ind)], 112.3 [CH (Ind)], 117.4 [C (Pbf)], 119.2, 119.7, 122.3, 124.3 [4CH (Ind)], 125.2 [C (Pbf)], 128.4 [C (Ind)], 132.8, 135.6 [2C (Pbf)], 137.6 [C (Ind)], 138.7 [C $(\mathrm{Pbf})], 157.2\left[\mathrm{C}\left(\mathrm{NHC}\left(\mathrm{NH}_{2}\right)=\mathrm{N}\right)\right], 158.8$ [C $\left.(\mathrm{Pbf})\right], 161.0\left(\mathrm{C}_{3}\right), 170.2\left(\mathrm{C}_{6}\right)$; ES-MS m/z 636.6 $[\mathrm{M}+1]^{+}$; Anal. calcd. for $\mathrm{C}_{32} \mathrm{H}_{41} \mathrm{~N}_{7} \mathrm{O}_{5} \mathrm{~S}: \mathrm{C}, 60.45 ; \mathrm{H}, 6.50 ; \mathrm{N}, 15.42$. Found: $\mathrm{C}, 60.59 ; \mathrm{H}, 6.24$; N, 15.63 .

\subsection{3. (1S,5S,8aR)-5-(3-(2-((2,2,4,6,7-Pentamethyl-2,3-dihydrobenzofuran-5-yl)sulfonyl)-} guanidino)propyl)-1-phenylmethyl-3,6-dioxooctahydroimidazo[1,5-a]pyrazine [(R)-9a]

Foam (94 mg, 51\%); $[\alpha]_{\mathrm{D}}{ }^{20}+4.1\left(c 1.0, \mathrm{CH}_{2} \mathrm{Cl}_{2}\right)$; HPLC [Sunfire $\mathrm{C}_{18}(4.6 \times 150 \mathrm{~mm}, 3.5$ $\mu \mathrm{m}), 10-100 \%$ gradient of solvent $\mathrm{A}$ in $30 \mathrm{~min}] t_{\mathrm{R}} 20.41 \mathrm{~min} ;{ }^{1} \mathrm{H} \mathrm{NMR}\left(400 \mathrm{MHz},\left(\mathrm{CD}_{3}\right)_{2} \mathrm{CO}\right)$ $\delta$ (ppm): 1.44 [s, 6H, 2CH $(\mathrm{Pbf})], 1.64$ [m, 2H, $\gamma-\mathrm{H}(\mathrm{Arg})], 1.90$ [m, 2H, $\beta-\mathrm{H}(\mathrm{Arg})], 2.04$ [s, $3 \mathrm{H}, \mathrm{CH}_{3}$ (Pbf)], 2.49 [s, 3H, $\mathrm{CH}_{3}$ (Pbf)], 2.57 [s, 3H, $\mathrm{CH}_{3}$ (Pbf)], 2.87 (m, 2H, 1- $\mathrm{CH}_{2}$ ), 2.98 [s, 2H, $\mathrm{CH}_{2}$ (Pbf)], 3.19 [m, 2H, $\delta$-H (Arg)], 3.27 (ddd, 1H, $J=4,5$ and $\left.11.5 \mathrm{~Hz}, 8-\mathrm{H}\right), 3.65$ (t, $1 \mathrm{H}, J=11.5 \mathrm{~Hz}, 8-\mathrm{H}), 3.97(\mathrm{ddd}, 1 \mathrm{H}, J=4,7$ and $11.5 \mathrm{~Hz}, 8 \mathrm{a}-\mathrm{H}), 4.13(\mathrm{dd}, 1 \mathrm{H}, J=4$ and $9.5 \mathrm{~Hz}, 5-\mathrm{H}), 4.29$ (q, 1H, $J=7 \mathrm{~Hz}, 1-\mathrm{H}), 5.86(\mathrm{~m}, 1 \mathrm{H}, 2-\mathrm{H}), 6.50\left[\mathrm{~m}, 3 \mathrm{H}, \mathrm{NHC}\left(\mathrm{NH}_{2}\right)=\mathrm{N}\right]$, $7.13(\mathrm{~m}, 1 \mathrm{H}, \mathrm{Ph}), 7.14(\mathrm{~d}, 1 \mathrm{H}, J=5 \mathrm{~Hz}, 1-\mathrm{H}), 7.28-7.35$ (m, 4H, Ph); ${ }^{13} \mathrm{C} \mathrm{NMR}(100 \mathrm{MHz}$, $\left.\left(\mathrm{CD}_{3}\right)_{2} \mathrm{CO}\right) \delta(\mathrm{ppm}):$ 13.2, 18.8, $20.3\left[3 \mathrm{CH}_{3}(\mathrm{Pbf})\right], 26.9\left[\mathrm{C}_{\gamma}(\mathrm{Arg})\right], 29.4$ [2 $\left.\mathrm{CH}_{3}(\mathrm{Pbf})\right], 30.6$ $\left[\mathrm{C}_{\beta}(\mathrm{Arg})\right], 36.4\left[1-\mathrm{CH}_{2}\right], 41.7\left(\mathrm{C}_{8}\right), 41.8\left[\mathrm{C}_{\delta}(\mathrm{Arg})\right], 44.0\left[\mathrm{CH}_{2}(\mathrm{Pbf})\right], 52.8\left(\mathrm{C}_{8 \mathrm{a}}\right), 54.4\left(\mathrm{C}_{5}\right)$, $55.4\left(\mathrm{C}_{1}\right), 87.8,118.1,126.0$ [3C (Pbf)], 128.1, 130.2, 130.4 [5CH (Ph)], 133.5, 136.6 [2C $(\mathrm{Pbf})], 139.4[\mathrm{C}(\mathrm{Pbf})$ and $\mathrm{C}(\mathrm{Ph})], 158.0\left[\mathrm{C}\left(\mathrm{NHC}\left(\mathrm{NH}_{2}\right)=\mathrm{N}\right)\right], 159.5[\mathrm{C}(\mathrm{Pbf})], 162.3\left(\mathrm{C}_{3}\right)$, $170.6\left(\mathrm{C}_{6}\right)$; ES-MS m/z $597.5[\mathrm{M}+1]^{+}$; Anal. calcd. for $\mathrm{C}_{30} \mathrm{H}_{40} \mathrm{~N}_{6} \mathrm{O}_{5} \mathrm{~S}: \mathrm{C}, 60.38 ; \mathrm{H}, 6.76 ; \mathrm{N}$, 14.08. Found: C, 60.12; H, 6.47; N, 14.20. 


\subsection{4. (1S,5S,8aS)-5-(3-(2-((2,2,4,6,7-Pentamethyl-2,3-dihydrobenzofuran-5-yl)sulfonyl)-}

guanidino)propyl)-1-phenylmethyl-3,6-dioxooctahydroimidazo[1,5-a]pyrazine [(S)-9a]

Foam (111 mg, 60\%); $[\alpha]_{\mathrm{D}}{ }^{20}-9.9\left(c 1.2, \mathrm{CH}_{2} \mathrm{Cl}_{2}\right)$; HPLC [Sunfire $\mathrm{C}_{18}(4.6 \times 150 \mathrm{~mm}, 3.5$ $\mu \mathrm{m}), 10-100 \%$ gradient of solvent $\mathrm{A}$ in $30 \mathrm{~min}] t_{\mathrm{R}} 21.31 \mathrm{~min} ;{ }^{1} \mathrm{H} \mathrm{NMR}\left(400 \mathrm{MHz},\left(\mathrm{CD}_{3}\right)_{2} \mathrm{CO}\right)$ $\delta(\mathrm{ppm}): 1.44$ [s, 6H, 2CH 3 (Pbf)], 1.45 [m, 2H, $\gamma-\mathrm{H}(\mathrm{Arg})], 1.66$ [m, 1H, $\beta-\mathrm{H}(\mathrm{Arg})], 1.97$ [m, $1 \mathrm{H}, \beta-\mathrm{H}(\mathrm{Arg})], 2.05$ [s, 3H, $\left.\mathrm{CH}_{3}(\mathrm{Pbf})\right], 2.42$ [m, 1H, $\left.\delta-\mathrm{H}(\mathrm{Arg})\right], 2.50$ [s, 3H, $\left.\mathrm{CH}_{3}(\mathrm{Pbf})\right]$, 2.57 [s, 3H, $\mathrm{CH}_{3}$ (Pbf)], 2.74 (ddd, $1 \mathrm{H}, J=3.5,5.5$ and $\left.12 \mathrm{~Hz}, 8-\mathrm{H}\right), 2.87\left(\mathrm{~m}, 1 \mathrm{H}, 1-\mathrm{CH}_{2}\right.$ ), $2.99\left[\mathrm{~s}, 2 \mathrm{H}, \mathrm{CH}_{2}(\mathrm{Pbf})\right], 3.07\left(\mathrm{~m}, 2 \mathrm{H}, 8-\mathrm{H}\right.$ and 1- $\left.\mathrm{CH}_{2}\right), 3.17$ [m, 1H, $\left.\delta-\mathrm{H}(\mathrm{Arg})\right], 3.51(\mathrm{dt}, 1 \mathrm{H}$, $J=3.5$ and $10 \mathrm{~Hz}, 8 \mathrm{a}-\mathrm{H}), 3.77(\mathrm{ddd}, 1 \mathrm{H}, J=6,7.5$ and $10 \mathrm{~Hz}, 1-\mathrm{H}), 3.86(\mathrm{dd}, 1 \mathrm{H}, J=3$ and 6 $\mathrm{Hz}, 5-\mathrm{H}), 6.01(\mathrm{~m}, 1 \mathrm{H}, 2-\mathrm{H}), 6.30\left[\mathrm{~m}, 1 \mathrm{H}, \mathrm{NHC}\left(\mathrm{NH}_{2}\right)=\mathrm{N}\right], 6.60\left[\mathrm{~m}, 2 \mathrm{H}, \mathrm{NHC}\left(\mathrm{NH}_{2}\right)=\mathrm{N}\right], 7.13$ $(\mathrm{d}, 1 \mathrm{H}, J=5.5 \mathrm{~Hz}, 1-\mathrm{H}), 7.21-7.38(\mathrm{~m}, 5 \mathrm{H}, \mathrm{Ph}) ;{ }^{13} \mathrm{C} \mathrm{NMR}\left(100 \mathrm{MHz},\left(\mathrm{CD}_{3}\right)_{2} \mathrm{CO}\right) \delta(\mathrm{ppm})$ : 11.9, 17.5, $18.9\left[3 \mathrm{CH}_{3}(\mathrm{Pbf})\right], 24.2\left[\mathrm{C}_{\gamma}(\mathrm{Arg})\right], 27.7\left[\mathrm{C}_{\beta}(\mathrm{Arg})\right], 28.8\left[2 \mathrm{CH}_{3}(\mathrm{Pbf})\right], 40.2[1-$ $\left.\mathrm{CH}_{2}\right], 40.8\left[\mathrm{C}_{\delta}(\mathrm{Arg})\right], 43.0\left[\mathrm{CH}_{2}(\mathrm{Pbf})\right], 44.0\left(\mathrm{C}_{8}\right), 57.2\left(\mathrm{C}_{5}\right), 56.0\left(\mathrm{C}_{1}\right), 58.7\left(\mathrm{C}_{8 \mathrm{a}}\right), 86.3$, 116.7, 124.6 [3C (Pbf)], 126.9, 128.8, 129.5 [5CH (Ph)], 132.1, 134.9 [2C (Pbf)], 137.4 [C

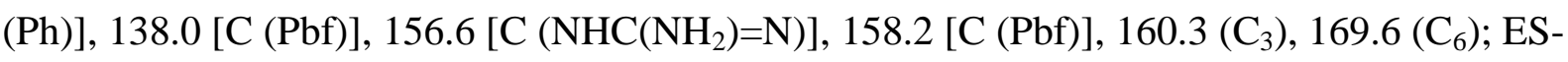
MS $m / z$ 597.6 [M+1] $]^{+}$; Anal. calcd. for $\mathrm{C}_{30} \mathrm{H}_{40} \mathrm{~N}_{6} \mathrm{O}_{5} \mathrm{~S}: \mathrm{C}, 60.38 ; \mathrm{H}, 6.76 ; \mathrm{N}, 14.08$. Found: $\mathrm{C}$, $60.45 ; \mathrm{H}, 6.50 ; \mathrm{N}, 13.93$.

4.5 General procedure for the $N_{4}$-alkylation of 2-oxopiperazines $\mathbf{6} \boldsymbol{a}, \boldsymbol{b}$ and $7 \boldsymbol{a}$. Synthesis of the 4-alkyl-2-oxopiperazines (R)- and (S)-(10a,b-13a)

DIEA $(456 \mu \mathrm{L}, 2.71 \mathrm{mmol})$ and benzyl bromide $(541 \mu \mathrm{L}, 2.71 \mathrm{mmol})$ or tert-butyl bromoacetate $(540 \mu \mathrm{L}, 2.71 \mathrm{mmol})$ were added under argon in four equal portions over $72 \mathrm{~h}$ to a solution of the corresponding 2-oxopiperazine (R)- and (S)-6a,b and -7a (0.66 mmol) in anhydrous $\mathrm{CH}_{3} \mathrm{CN}(10 \mathrm{~mL})$ at $60^{\circ} \mathrm{C}$. After 4 days of stirring, the solvent was removed under reduced pressure and the residue was dissolved in $\mathrm{CH}_{2} \mathrm{Cl}_{2}(100 \mathrm{~mL})$. The solution was successively washed with $\mathrm{H}_{2} \mathrm{O}(2 \times 25 \mathrm{~mL})$ and brine $(25 \mathrm{~mL})$, dried over $\mathrm{Na}_{2} \mathrm{SO}_{4}$ and evaporated to dryness. The residue was purified by flash chromatography, using $\mathrm{MeOH}$ in $\mathrm{CH}_{2} \mathrm{Cl}_{2}$ gradient as mobile phase to obtain the respective 4-benzyl-2-oxopiperazines (R)- and (S)-10a,b-13a.

4.5.1. (5R,3S)-4-Benzyl-5-((S)-1-((tert-butoxycarbonyl)amino-2-(1-(tert-butoxycarbonyl)indol-3-yl)ethyl-3-(3-(2-((2,2,4,6,7-pentamethyl-2,3-dihydrobenzofuran-5yl)sulfonyl)guanidino)propyl)-2-oxopiperazine [(R)-10a] 
Reddish solid (446 mg, 75\%); $[\alpha]_{\mathrm{D}}{ }^{20}-48 \quad\left(c \quad 1.5, \mathrm{CH}_{2} \mathrm{Cl}_{2}\right) ; \mathrm{Mp}: 106-108 \quad{ }^{\circ} \mathrm{C}$ (EtOAc/hexane); HPLC [Sunfire $\mathrm{C}_{18}(4.6 \times 150 \mathrm{~mm}, 3.5 \mu \mathrm{m}), 80-100 \%$ gradient of solvent A in $\mathrm{B}, 30 \mathrm{~min}] t_{\mathrm{R}} 7.35 \mathrm{~min} ;{ }^{1} \mathrm{H}$ NMR (400 MHz, $\left.\left(\mathrm{CD}_{3}\right)_{2} \mathrm{CO}\right) \delta(\mathrm{ppm}): 1.29$ (s, 9H, Boc), 1.42 [s, $\left.6 \mathrm{H}, 2 \mathrm{CH}_{3}(\mathrm{Pbf})\right], 1.65$ [s, 3H, Boc (Ind)], 1.82 [m, 4H, $\beta-\mathrm{H}$ and $\left.\gamma-\mathrm{H}(\mathrm{Arg})\right], 2.02$ [s, 3H, $\mathrm{CH}_{3}$ (Pbf)], 2.49 [s, 3H, $\mathrm{CH}_{3}$ (Pbf)], 2.57 [s, 3H, $\mathrm{CH}_{3}$ (Pbf)], 2.88 (m, 1H, CH $\mathrm{CH}_{2}$-Ind), 2.92 [s, 2H, $\left.\mathrm{CH}_{2}(\mathrm{Pbf})\right], 3.03$ [m, 1H, 3-H], 3.06 [m, 2H, $\left.\delta-\mathrm{H}(\mathrm{Arg})\right], 3.49(\mathrm{~m}, 1 \mathrm{H}, 5-\mathrm{H}), 3.50$ (m, 1H, 6$\mathrm{H}), 3.61\left[\mathrm{~m}, 3 \mathrm{H}, \mathrm{CH}_{2}-\mathrm{Ind}, 4-\mathrm{CH}_{2}(\mathrm{Bn})\right.$ and 6-H], $4.20\left[\mathrm{~m}, 2 \mathrm{H}, 5-\mathrm{CH}\right.$ and $\left.4-\mathrm{CH}_{2}(\mathrm{Bn})\right], 6.10$ (d, 1H, $J=9.5 \mathrm{~Hz}, N H \mathrm{Boc}), 6.47\left[\mathrm{~m}, 3 \mathrm{H}, \mathrm{NHC}\left(\mathrm{NH}_{2}\right)=\mathrm{N}\right], 6.97(\mathrm{~s}, 1 \mathrm{H}, 1-\mathrm{H}), 7.15(\mathrm{t}, 1 \mathrm{H}, J=$ $7.5 \mathrm{~Hz}, \mathrm{Ar}), 7.28$ (m, 4H, Ar), 7.51 (m, 2H, Ar), 7.53 (m, 2H, Ar), 8.12 (d, 1H, J = $7.5 \mathrm{~Hz}$, Ind). ${ }^{13} \mathrm{C}$ NMR $\left(100 \mathrm{MHz},\left(\mathrm{CD}_{3}\right)_{2} \mathrm{CO}\right) \delta(\mathrm{ppm}): 12.5,18.2,19.5\left[3 \mathrm{CH}_{3}(\mathrm{Pbf})\right], 27.1\left[\mathrm{C}_{\gamma}\right.$ (Arg)], $28.2\left[\mathrm{CH}_{2}\right.$-Ind and $2 \mathrm{CH}_{3}$ (Pbf)], $28.3\left[3 \mathrm{CH}_{3}\right.$ (Boc)], $28.5\left[\mathrm{C}_{\beta}(\mathrm{Arg})\right], 28.7\left[3 \mathrm{CH}_{3}\right.$ (Boc)], $41.3\left[\mathrm{C}_{\delta}(\mathrm{Arg})\right], 41.5\left(\mathrm{C}_{6}\right), 43.6\left[\mathrm{CH}_{2}(\mathrm{Pbf})\right], 51.4\left[\mathrm{C}_{5}-\mathrm{CH}\right], 51.8$ [4- $\left.\mathrm{CH}_{2}(\mathrm{Bn})\right], 55.4$ $\left(\mathrm{C}_{5}\right), 61.9\left(\mathrm{C}_{3}\right), 78.8,83.9$ [2C (2Boc)], 86.9 [C (Pbf)], 115.8 [CH (Ar)], 117.4 [C (Pbf)], 118.8 [C (Ar)], 119.9, 123.2, 124.4, 124.9 [4CH (Ar)], 125.3 [C (Pbf)], 128.1, 129.3, 129.8 [5CH (Ar)], 132.0 [C (Ar)], 132.8, 135.6 [2C (Pbf)], 136.4 [C (Ar)], 138.7 [C (Pbf)], 139.6 [C (Ar)], 150.3, 156.6 [2CO (2Boc)], $157.4\left[\mathrm{C}\left(\mathrm{NHC}\left(\mathrm{NH}_{2}\right)=\mathrm{N}\right)\right], 158.9[\mathrm{C}(\mathrm{Pbf})], 172.1\left(\mathrm{C}_{2}\right)$; ES-MS m/z $901.1[\mathrm{M}+1]^{+}$; Anal. calcd. for $\mathrm{C}_{48} \mathrm{H}_{65} \mathrm{~N}_{7} \mathrm{O}_{8} \mathrm{~S}$ : C, 64.05; H, 7.28; N, 10.89. Found: C, 64.23; H, 7.05; N, 11.13.

\subsection{2. (5S,3S)-4-Benzyl-5-((S)-1-((tert-butoxycarbonyl)amino-2-(1-(tert-butoxycarbonyl)-} indol-3-yl)ethyl-3-(3-(2-((2,2,4,6,7-pentamethyl-2,3-dihydrobenzofuran-5-yl)sulfonyl)guanidino)propyl)-2-oxopiperazine [(S)-10a]

Reddish solid (357 mg, 60\%); $[\alpha]_{\mathrm{D}}{ }^{20}-14 \quad\left(c \quad 1.1, \mathrm{CH}_{2} \mathrm{Cl}_{2}\right) ; \mathrm{Mp}: 112-114{ }^{\circ} \mathrm{C}$ (EtOAc/hexane); HPLC [Sunfire $\mathrm{C}_{18}(4.6 \times 150 \mathrm{~mm}, 3.5 \mu \mathrm{m}), 80-100 \%$ gradient of solvent A in $\mathrm{B}, 30 \mathrm{~min}] t_{\mathrm{R}} 7.32 \mathrm{~min} ;{ }^{1} \mathrm{H}$ NMR $\left(400 \mathrm{MHz},\left(\mathrm{CD}_{3}\right)_{2} \mathrm{CO}\right) \delta(\mathrm{ppm}): 1.33$ (s, 9H, Boc), 1.43 [s, $\left.6 \mathrm{H}, 2 \mathrm{CH}_{3}(\mathrm{Pbf})\right], 1.44$ [m, 2H, $\gamma-\mathrm{H}$ (Arg)], 1.55 [m, 3H, $\beta-\mathrm{H}$ (Arg)], 1.66 [s, 3H, Boc (Ind)], 2.03 [s, 3H, $\left.\mathrm{CH}_{3}(\mathrm{Pbf})\right], 2.47$ [s, 3H, $\left.\mathrm{CH}_{3}(\mathrm{Pbf})\right], 2.55$ [s, 3H, $\left.\mathrm{CH}_{3}(\mathrm{Pbf})\right], 2.86$ (m, 1H, $\mathrm{CH}_{2^{-}}$ Ind), 2.94 [s, 2H, $\mathrm{CH}_{2}$ (Pbf)], $2.97[\mathrm{~m}, 1 \mathrm{H}, \delta-\mathrm{H}(\mathrm{Arg})], 3.06$ [m, 2H, $\delta-\mathrm{H}(\mathrm{Arg})$ and 3-H], 3.14 (m, 1H, 5-H), 3.16 [m, 3H, $\mathrm{CH}_{2}$-Ind], $3.47(\mathrm{~m}, 1 \mathrm{H}, 6-\mathrm{H}), 3.60(\mathrm{~m}, 1 \mathrm{H}, 6-\mathrm{H}), 3.76$ [d, 1H, $J=$ $\left.14 \mathrm{~Hz}, 4-\mathrm{CH}_{2}(\mathrm{Bn})\right], 4.05$ [d, 1H, $\left.J=14 \mathrm{~Hz}, 4-\mathrm{CH}_{2}(\mathrm{Bn})\right], 4.14$ [m, 2H, 5-CH], 6.18 (d, 1H, $J$ $=9 \mathrm{~Hz}, N H B o c), 6.40\left[\mathrm{~m}, 3 \mathrm{H}, \mathrm{NHC}\left(\mathrm{NH}_{2}\right)=\mathrm{N}\right], 7.17(\mathrm{~s}, 1 \mathrm{H}, 1-\mathrm{H}), 7.24-7.55(\mathrm{~m}, 8 \mathrm{H}, \mathrm{Ar}), 7.64$ $(\mathrm{d}, 1 \mathrm{H}, J=7.5 \mathrm{~Hz}, \mathrm{Ar}), 8.13(\mathrm{~d}, 1 \mathrm{H}, J=7.5 \mathrm{~Hz}, \mathrm{Ind}) ;{ }^{13} \mathrm{C} \mathrm{NMR}\left(100 \mathrm{MHz},\left(\mathrm{CD}_{3}\right)_{2} \mathrm{CO}\right) \delta$

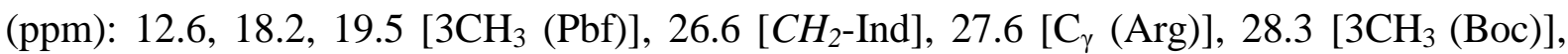


$28.6\left[2 \mathrm{CH}_{3}(\mathrm{Pbf})\right], 28.7\left[3 \mathrm{CH}_{3}(\mathrm{Boc})\right], 31.4\left[\mathrm{C}_{\beta}(\mathrm{Arg})\right], 40.3\left(\mathrm{C}_{6}\right), 41.3\left[\mathrm{C}_{\delta}(\mathrm{Arg})\right], 43.6\left[\mathrm{CH}_{2}\right.$ $(\mathrm{Pbf})], 53.5$ [C $5^{-} \mathrm{CH}$ ], 62.3 [4- $\left.\mathrm{CH}_{2}(\mathrm{Bn})\right], 63.4\left(\mathrm{C}_{5}\right), 63.5\left(\mathrm{C}_{3}\right), 78.9,84.0$ [2C (2Boc)], 86.9 [C (Pbf)], 115.9 [CH (Ar)], 117.4 [C (Pbf)], 118.9 [C (Ar)], 120.0, 123.2, 124.1, 125.0 [4CH (Ar)], 125.2 [C (Pbf)], 128.1, 129.1, 130.3 [5CH (Ar)], 131.9 [C (Ar)], 132.8, 135.7 [2C (Pbf)], 136.3 [C (Ar)], 138.7 [C (Pbf)], 140.0 [C (Ar)], 150.3, 156.7 [2CO (2Boc)], 157.3 [C $\left.\left(\mathrm{NHC}\left(\mathrm{NH}_{2}\right)=\mathrm{N}\right)\right], 158.9[\mathrm{C}(\mathrm{Pbf})], 173.4\left(\mathrm{C}_{2}\right)$; ES-MS m/z $901.1[\mathrm{M}+1]^{+}$; Anal. calcd. for $\mathrm{C}_{48} \mathrm{H}_{65} \mathrm{~N}_{7} \mathrm{O}_{8} \mathrm{~S}: \mathrm{C}, 64.05 ; \mathrm{H}, 7.28 ; \mathrm{N}, 10.89$. Found: C, 63.87; H, 7.03; N, 11.24.

\subsection{3. (5R,3S)-4-Benzyl-5-((S)-1-((tert-butoxycarbonyl)amino-2-(1-(tert-butoxycarbonyl)-} indol-3-yl)ethyl-3-(4-((tert-butoxycarbonyl)amino)butyl)-2-oxopiperazine [(R)-10b]

Reddish solid (333 mg, 70\%); $[\alpha]_{\mathrm{D}}{ }^{20}+0.9$ (c 1.1, $\mathrm{CH}_{2} \mathrm{Cl}_{2}$ ); Mp: 76-78 ${ }^{\circ} \mathrm{C}$ (EtOAc/hexane); HPLC [Sunfire $\mathrm{C}_{18}(4.6 \times 150 \mathrm{~mm}, 3.5 \mu \mathrm{m}), 70-100 \%$ gradient of solvent A in B, $30 \mathrm{~min}$ ] $t_{\mathrm{R}}$ $11.27 \mathrm{~min} ;{ }^{1} \mathrm{H}$ NMR (400 MHz, $\left.\left(\mathrm{CD}_{3}\right)_{2} \mathrm{CO}\right) \delta$ (ppm): 1.29 [m, 2H, $\gamma-\mathrm{H}$ (Lys)], 1.30 (s, 9H, Boc), 1.34 [m, 2H, $\delta$-H (Lys)], 1.36 [s, 9H, Boc (Lys)], 1.61 [m, 1H, $\beta-\mathrm{H}$ (Lys)], 1.66 [s, 9H, Boc (Ind)], 1.72 [m, 1H, $\beta$-H (Lys)], 1.83 [m, 1H, $\varepsilon-\mathrm{H}$ (Lys)], 2.88 (dd, 1H, $J=11$ and 15 Hz, $\mathrm{CH}_{2}$-Ind), 3.00 [m, 1H, ع-H (Lys)], 3.04 (m, 1H, 3-H), 3.49 (m, 1H, 5-H), 3.50 (m, 1H, 6-H), $3.60\left[\mathrm{~m}, 2 \mathrm{H}, 4-\mathrm{CH}_{2}(\mathrm{Bn})\right.$ and 6-H], $3.63\left(\mathrm{~m}, 1 \mathrm{H}, \mathrm{CH}_{2}-\mathrm{Ind}\right), 4.22\left[\mathrm{~m}, 1 \mathrm{H}, 4-\mathrm{CH}_{2}(\mathrm{Bn})\right], 4.23$ (m, 1H, 5-CH), 5.80 [m, 1H, NHBoc (Lys)], 6.08 (d, 1H, J = 9.5 Hz, NHBoc), 6.82 (s, 1H, 1H), 7.18 (t, 1H, J=7.5 Hz, Ar), 7.29 (m, 2H, Ar), 7.52 (m, 2H, Ar), 7.53 (m, 4H, Ar), 8.12 (d, $1 \mathrm{H}, J=7.5 \mathrm{~Hz}, \mathrm{Ar}) .{ }^{13} \mathrm{C} \mathrm{NMR}\left(100 \mathrm{MHz},\left(\mathrm{CD}_{3}\right)_{2} \mathrm{CO}\right) \delta$ (ppm): $24.5\left[\mathrm{C}_{\gamma}(\mathrm{Lys})\right], 28.2\left[\mathrm{CH}_{2^{-}}\right.$

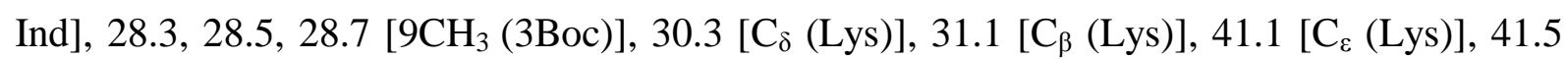
$\left(\mathrm{C}_{6}\right), 51.3\left[\mathrm{C}_{5}-\mathrm{CH}\right], 51.9$ [4- $\left.\mathrm{CH}_{2}(\mathrm{Bn})\right], 55.4\left(\mathrm{C}_{5}\right), 62.2\left(\mathrm{C}_{3}\right), 78.8,83.9$ [3C (3Boc)], 115.8 [CH (Ar)], 118.8 [C (Ar)], 119.9, 123.2, 124.3, 124.9, 128.0, 129.3, 129.9 [9CH (Ar)], 132.0, 136.4, 139.7 [3C (Ar)], 150.3, 156.6 [3CO (3Boc)], $172.0\left(\mathrm{C}_{2}\right)$; ES-MS m/z $720.9[\mathrm{M}+1]^{+}$; Anal. calcd. for $\mathrm{C}_{40} \mathrm{H}_{57} \mathrm{~N}_{5} \mathrm{O}_{7}$ : C, 66.73; H, 7.98; N, 9.73. Found: C, 66.51; H, 8.15; N, 9.95.

\subsection{4. (5S,3S)-4-Benzyl-5-((S)-1-((tert-butoxycarbonyl)amino-2-(1-(tert-butoxycarbonyl)-} indol-3-yl)ethyl-3-(4-((tert-butoxycarbonyl)amino)butyl)-2-oxopiperazine [(S)-10b]

Reddish solid (381 mg, 80\%); $[\alpha]_{\mathrm{D}}{ }^{20}+13.6$ (c 1.2, $\left.\mathrm{CH}_{2} \mathrm{Cl}_{2}\right) ; \mathrm{Mp}: \quad 84-86{ }^{\circ} \mathrm{C}$ (EtOAc/hexane); HPLC [Sunfire $\mathrm{C}_{18}(4.6 \times 150 \mathrm{~mm}, 3.5 \mu \mathrm{m}), 70-100 \%$ gradient of solvent A in B $30 \mathrm{~min}] t_{\mathrm{R}} 11.86 \mathrm{~min} ;{ }^{1} \mathrm{H} \mathrm{NMR}\left(400 \mathrm{MHz},\left(\mathrm{CD}_{3}\right)_{2} \mathrm{CO}\right) \delta(\mathrm{ppm}): 1.21$ [m, 2H, $\gamma-\mathrm{H}$ (Lys)], $1.31[\mathrm{~m}, 2 \mathrm{H}, \delta-\mathrm{H}$ (Lys)], 1.36 [m, 19H, Boc, Boc (Lys) and $\beta-\mathrm{H}$ (Lys)], 1.67 [s, 9H, Boc (Ind)], 1.68 [m, 1H, $\beta$-H (Lys)], 2.92 (m, 1H, $\mathrm{CH}_{2}$-Ind), 2.94 [m, 2H, $\varepsilon-\mathrm{H}$ (Lys)], 3.07 (t, 1H, 
$J=7 \mathrm{~Hz}, 3-\mathrm{H}), 3.14(\mathrm{~m}, 1 \mathrm{H}, 5-\mathrm{H}), 3.24$ (dd, $1 \mathrm{H}, J=2.5$ and $\left.15 \mathrm{~Hz}, \mathrm{CH}_{2}-\mathrm{Ind}\right), 3.53$ (m, 2H, 6H), $3.80\left[\mathrm{~d}, 1 \mathrm{H}, J=14 \mathrm{~Hz}, 4-\mathrm{CH}_{2}(\mathrm{Bn})\right], 4.08$ [d, 1H, $\left.J=14 \mathrm{~Hz}, 4-\mathrm{CH}_{2}(\mathrm{Bn})\right], 4.17$ (m, 1H, 5$\mathrm{CH}), 5.82[\mathrm{~m}, 1 \mathrm{H}, N H \mathrm{Boc}(\mathrm{Lys})], 6.13$ (d, 1H, J = 8.5 Hz, NHBoc), 7.03 (s, 1H, 1-H), 7.26 (m, 2H, Ar), 7.34 (m, 3H, Ar), 7.51 (m, 3H, Ar), 7.66 (d, 1H, J=7.5 Hz, Ar), 8.14 (d, 1H, $J=$ $7.5 \mathrm{~Hz}, \mathrm{Ar}) .{ }^{13} \mathrm{C}$ NMR (100 MHz, $\left.\left(\mathrm{CD}_{3}\right)_{2} \mathrm{CO}\right) \delta(\mathrm{ppm}): 24.1\left[\mathrm{C}_{\gamma}\right.$ (Lys)], 26.6 [ $\mathrm{CH}_{2}$-Ind], 28.3, $28.7\left[9 \mathrm{CH}_{3}\right.$ (3Boc)], $30.0\left[\mathrm{C}_{\delta}\right.$ (Lys)], $34.4\left[\mathrm{C}_{\beta}\right.$ (Lys)], $40.2\left(\mathrm{C}_{6}\right), 40.9\left[\mathrm{C}_{\varepsilon}(\mathrm{Lys})\right], 52.9$ [C $5^{-}$ $\mathrm{CH}$ ], 62.4 [4- $\left.\mathrm{CH}_{2}(\mathrm{Bn})\right], 63.9\left(\mathrm{C}_{5}\right), 64.0\left(\mathrm{C}_{3}\right), 78.2,78.8,84.0$ [3C (3Boc)], 115.9 [CH (Ar)], 119.0 [C (Ar)], 120.0, 123.2, 124.0, 125.0, 128.0, 129.1, 130.2 [9CH (Ar)], 131.9, 136.4, 140.2 [3C (Ar)], 150.3, 156.6 [3CO (3Boc)], $173.2\left(\mathrm{C}_{2}\right)$; ES-MS m/z 720.9 [M+1] ${ }^{+}$; Anal. calcd. for $\mathrm{C}_{40} \mathrm{H}_{57} \mathrm{~N}_{5} \mathrm{O}_{7}$ : C, 66.73; H, 7.98; N, 9.73. Found: C, 66.49; H, 8.21; N, 9.51.

4.5.5. (5R,3S)-4-Benzyl-5-((S)-1-(tert-butoxycarbonyl)amino-2-phenylethyl)-3-(3-(2((2,2,4,6,7-pentamethyl-2,3-dihydrobenzofuran-5-yl)sulfonyl)guanidino) propyl)-2oxopiperazine [(R)-11a]

Reddish solid (401 mg, 80\%); $[\alpha]_{\mathrm{D}}{ }^{20}-25 \quad\left(c \quad 1.0, \mathrm{CH}_{2} \mathrm{Cl}_{2}\right) ; \mathrm{Mp}: 102-104{ }^{\circ} \mathrm{C}$ (EtOAc/hexane); HPLC [Sunfire $\mathrm{C}_{18}(4.6 \times 150 \mathrm{~mm}, 3.5 \mu \mathrm{m}), 60-100 \%$ gradient of solvent A in $\mathrm{B}, 30 \mathrm{~min}] t_{\mathrm{R}} 16.05 \mathrm{~min} ;{ }^{1} \mathrm{H} \mathrm{NMR}\left(400 \mathrm{MHz},\left(\mathrm{CD}_{3}\right)_{2} \mathrm{CO}\right) \delta(\mathrm{ppm}): 1.28$ (s, 9H, Boc), 1.45 [s, 6H, 2CH $\left.\mathrm{CH}_{3}(\mathrm{Pbf})\right], 1.73[\mathrm{~m}, 4 \mathrm{H}, \beta-\mathrm{H}$ and $\gamma-\mathrm{H}(\mathrm{Arg})], 2.05$ [s, 3H, $\left.\mathrm{CH}_{3}(\mathrm{Pbf})\right], 2.51$ [s, 3H, $\left.\mathrm{CH}_{3}(\mathrm{Pbf})\right], 2.58$ [s, 3H, $\left.\mathrm{CH}_{3}(\mathrm{Pbf})\right], 2.78$ (dd, $1 \mathrm{H}, J=11$ and $\left.14 \mathrm{~Hz}, \mathrm{CH}_{2}-\mathrm{Ph}\right), 2.98$ [s, $2 \mathrm{H}$, $\left.\mathrm{CH}_{2}(\mathrm{Pbf})\right], 3.00[\mathrm{~m}, 2 \mathrm{H}, \delta-\mathrm{H}(\mathrm{Arg})], 3.06[\mathrm{~m}, 1 \mathrm{H}, 3-\mathrm{H}], 3.40(\mathrm{~m}, 1 \mathrm{H}, 5-\mathrm{H}), 3.44(\mathrm{~m}, 1 \mathrm{H}, 6-$ H), $3.50\left(\mathrm{~m}, 1 \mathrm{H}, \mathrm{CH}_{2}-\mathrm{Ph}\right), 3.54\left[\mathrm{~m}, 1 \mathrm{H}, 4-\mathrm{CH}_{2}(\mathrm{Bn})\right], 3.60(\mathrm{~m}, 1 \mathrm{H}, 6-\mathrm{H}), 4.10(\mathrm{~m}, 1 \mathrm{H}, 5-\mathrm{CH})$, $4.21\left[\mathrm{~d}, 1 \mathrm{H}, J=13.5 \mathrm{~Hz}, 4-\mathrm{CH}_{2}(\mathrm{Bn})\right], 6.01$ (d, 1H, $\left.J=9.5 \mathrm{~Hz}, N H \mathrm{Boc}\right), 6.48$ [m, 3H, $\left.\mathrm{NHC}\left(\mathrm{NH}_{2}\right)=\mathrm{N}\right], 6.94(\mathrm{~s}, 1 \mathrm{H}, 1-\mathrm{H}), 7.14-7.52(\mathrm{~m}, 10 \mathrm{H}, \mathrm{Ar}) ;{ }^{13} \mathrm{C} \mathrm{NMR}\left(100 \mathrm{MHz},\left(\mathrm{CD}_{3}\right)_{2} \mathrm{CO}\right) \delta$ (ppm): 12.5, 18.2, 19.5 [3 $\left.\mathrm{CH}_{3}(\mathrm{Pbf})\right], 27.1\left[\mathrm{C}_{\gamma}(\mathrm{Arg})\right], 28.3\left[\mathrm{C}_{\beta}(\mathrm{Arg})\right], 28.5\left[3 \mathrm{CH}_{3}\right.$ (Boc)], $28.7\left[2 \mathrm{CH}_{3}(\mathrm{Pbf})\right], 38.6\left[\mathrm{CH}_{2}-\mathrm{Ph}\right], 41.2\left(\mathrm{C}_{6}\right), 41.4\left[\mathrm{C}_{\delta}(\mathrm{Arg})\right], 43.6\left[\mathrm{CH}_{2}(\mathrm{Pbf})\right], 51.7\left[4-\mathrm{CH}_{2}\right.$ $(\mathrm{Bn})$ ], $53.1\left[\mathrm{C}_{5}-\mathrm{CH}\right], 55.4\left(\mathrm{C}_{5}\right), 61.9\left(\mathrm{C}_{3}\right), 78.7$ [C (Boc)], 86.9, 117.5, 125.3 [3C (Pbf)], 126.7, 128.1, 128.9, 129.3, 129.9, 130.2 [10CH (Ar)], 132.8, 135.6, 138.7 [3C (Pbf)], 139.7

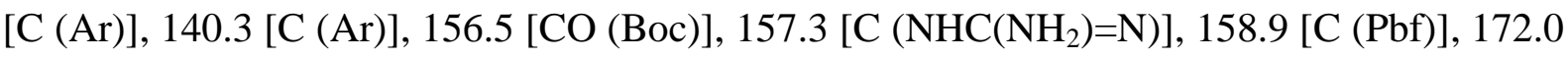
$\left(\mathrm{C}_{2}\right)$; ES-MS m/z $761.6[\mathrm{M}+1]^{+}$; Anal. calcd. for $\mathrm{C}_{41} \mathrm{H}_{56} \mathrm{~N}_{6} \mathrm{O}_{6} \mathrm{~S}: \mathrm{C}, 64.71 ; \mathrm{H}, 7.42 ; \mathrm{N}, 11.04$. Found: C, 64.48; H, 7.65; N, 11.25. 
4.5.6. (5S,3S)-4-Benzyl-5-((S)-1-(tert-butoxycarbonyl)amino-2-phenylethyl)-3-(3-(2((2,2,4,6,7-pentamethyl-2,3-dihydrobenzofuran-5-yl)sulfonyl)guanidino) propyl)-2oxopiperazine [(S)-11a]

Reddish solid (351 mg, 70\%); $[\alpha]_{\mathrm{D}}{ }^{20}+21 \quad\left(c \quad 1.0, \mathrm{CH}_{2} \mathrm{Cl}_{2}\right) ; \mathrm{Mp}: 100-102{ }^{\circ} \mathrm{C}$ (EtOAc/hexane); HPLC [Sunfire $\mathrm{C}_{18}(4.6 \times 150 \mathrm{~mm}, 3.5 \mu \mathrm{m}), 60-100 \%$ gradient of solvent A in $\mathrm{B}, 30 \mathrm{~min}] t_{\mathrm{R}} 16.01 \mathrm{~min} ;{ }^{1} \mathrm{H} \mathrm{NMR}\left(400 \mathrm{MHz},\left(\mathrm{CD}_{3}\right)_{2} \mathrm{CO}\right) \delta(\mathrm{ppm}): 1.27$ [m, 2H, $\left.\gamma-\mathrm{H}(\mathrm{Arg})\right]$, 1.30 (s, 9H, Boc), 1.43 [s, 6H, 2 $\mathrm{CH}_{3}$ (Pbf)], 1.59 [m, 2H, $\beta-\mathrm{H}$ (Arg)], 2.04 [s, 3H, $\mathrm{CH}_{3}$ (Pbf)], $2.48\left[\mathrm{~s}, 3 \mathrm{H}, \mathrm{CH}_{3}(\mathrm{Pbf})\right], 2.54$ [s, 3H, $\left.\mathrm{CH}_{3}(\mathrm{Pbf})\right], 2.68$ (dd, $1 \mathrm{H}, J=11$ and $\left.13.5 \mathrm{~Hz}, \mathrm{CH}_{2}-\mathrm{Ph}\right)$, $2.93[\mathrm{~m}, 1 \mathrm{H}, \delta-\mathrm{H}(\mathrm{Arg})], 2.97$ [s, 2H, $\left.\mathrm{CH}_{2}(\mathrm{Pbf})\right], 3.00(\mathrm{~m}, 1 \mathrm{H}, 5-\mathrm{H}), 3.01$ [m, 1H, $\delta-\mathrm{H}$ (Arg)], $3.06[\mathrm{~m}, 1 \mathrm{H}, 3-\mathrm{H}], 3.10\left(\mathrm{~m}, 1 \mathrm{H}, C_{2}-\mathrm{Ph}\right), 3.38(\mathrm{t}, 1 \mathrm{H}, J=13 \mathrm{~Hz}, 6-\mathrm{H}), 3.53(\mathrm{dt}, 1 \mathrm{H}, J=6$ and $13 \mathrm{~Hz}, 6-\mathrm{H}), 3.72$ [d, 1H, J = $\left.14 \mathrm{~Hz}, 4-\mathrm{CH}_{2}(\mathrm{Bn})\right], 3.99$ (m, 1H, 5-CH), 4.06 [m, 1H, 4- $\mathrm{CH}_{2}$ $(\mathrm{Bn})], 6.17(\mathrm{~d}, 1 \mathrm{H}, J=9 \mathrm{~Hz}, N H \mathrm{Boc}), 6.44\left[\mathrm{~m}, 3 \mathrm{H}, \mathrm{NHC}\left(\mathrm{NH}_{2}\right)=\mathrm{N}\right], 7.12-7.48(\mathrm{~m}, 11 \mathrm{H}, 1-\mathrm{H}$

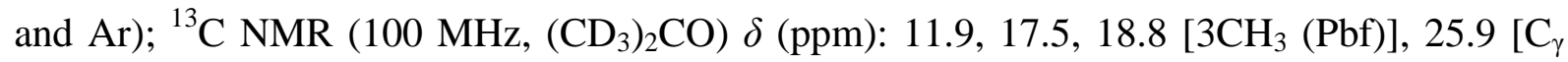

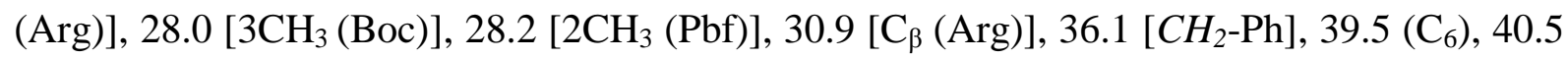
$\left[\mathrm{C}_{\delta}(\mathrm{Arg})\right], 42.9\left[\mathrm{CH}_{2}(\mathrm{Pbf})\right], 54.8\left(\mathrm{C}_{5}-\mathrm{CH}\right), 61.6\left[4-\mathrm{CH}_{2}(\mathrm{Bn})\right], 62.7\left(\mathrm{C}_{5}\right), 63.1\left(\mathrm{C}_{3}\right), 78.2[\mathrm{C}$ (Boc)], 86.2, 116.7, 124.6 [3C (Pbf)], 126.1, 127.4, 128.3, 128.4, 129.3, 129.6 [10CH (Ar)], 132.1, 134.9, 138.0 [3C (Pbf)], 139.5 [C (Ar)], 139.7 [C (Ar)], 155.9 [CO (Boc)], 156.6 [C $\left.\left(\mathrm{NHC}\left(\mathrm{NH}_{2}\right)=\mathrm{N}\right)\right], 158.2[\mathrm{C}(\mathrm{Pbf})], 172.6\left(\mathrm{C}_{2}\right)$; ES-MS m/z $761.6[\mathrm{M}+1]^{+}$; Anal. calcd. for $\mathrm{C}_{41} \mathrm{H}_{56} \mathrm{~N}_{6} \mathrm{O}_{6} \mathrm{~S}: \mathrm{C}, 64.71 ; \mathrm{H}, 7.42 ; \mathrm{N}, 11.04$. Found: C, 64.53; H, 7.21; N, 11.31.

4.5.7. (5R,3S)-5-((S)-1-((tert-Butoxycarbonyl)amino-2-(1-(tert-butoxycarbonyl)-indol-3yl)ethyl-4-(tert-butoxycarbonyl)methyl -3-(3-(2-((2,2,4,6,7-pentamethyl-2,3dihydrobenzofuran-5-yl)sulfonyl)guanidino)propyl)-2-oxopiperazine [(R)-12a]

Reddish solid (397 mg, 65\%); $[\alpha]_{\mathrm{D}}{ }^{20}+19$ (c 1.4, $\left.\mathrm{CH}_{2} \mathrm{Cl}_{2}\right) ; \mathrm{Mp}: 102-104{ }^{\circ} \mathrm{C}$ (EtOAc/hexane); HPLC [Sunfire $\mathrm{C}_{18}(4.6 \times 150 \mathrm{~mm}, 3.5 \mu \mathrm{m}), 80-100 \%$ gradient of solvent A in $\mathrm{B}, 30 \mathrm{~min}] t_{\mathrm{R}} 7.48 \mathrm{~min} ;{ }^{1} \mathrm{H}$ NMR (400 MHz, $\left.\left(\mathrm{CD}_{3}\right)_{2} \mathrm{CO}\right) \delta(\mathrm{ppm}): 1.28$ (s, 9H, Boc), 1.42 [s, $6 \mathrm{H}, 2 \mathrm{CH}_{3}$ (Pbf)], 1.47 (s, 9H, $\left.{ }^{\mathrm{B}} \mathrm{Bu}\right), 1.66$ [s, 3H, Boc (Ind)], 1.80 [m, 4H, $\beta-\mathrm{H}$ and $\gamma-\mathrm{H}$ (Arg)], $2.03\left[\mathrm{~s}, 3 \mathrm{H}, \mathrm{CH}_{3}\right.$ (Pbf)], 2.50 [s, 3H, $\left.\mathrm{CH}_{3}(\mathrm{Pbf})\right], 2.58$ [s, 3H, $\mathrm{CH}_{3}(\mathrm{Pbf})$ ], 2.80 (m, 1H, $\mathrm{CH}_{2^{-}}$ Ind), $2.93\left[\mathrm{~s}, 2 \mathrm{H}, \mathrm{CH}_{2}\right.$ (Pbf)], $3.20[\mathrm{~m}, 1 \mathrm{H}, 3-\mathrm{H}], 3.21\left(\mathrm{~m}, 1 \mathrm{H}, 4-\mathrm{CH}_{2}\right), 3.33[\mathrm{~m}, 2 \mathrm{H}, \delta-\mathrm{H}$ (Arg)], 3.40 (m, 1H, 5-H), 3.49 (m, 2H, 6-H), 3.60 (m, 1H, $\mathrm{CH}_{2}$-Ind), 3.64 (m, 1H, 4- $\mathrm{CH}_{2}$ ), $3.92(\mathrm{~m}, 1 \mathrm{H}, 5-\mathrm{CH}), 6.07(\mathrm{~d}, 1 \mathrm{H}, J=9.5 \mathrm{~Hz}, N H \mathrm{Boc}), 6.53\left[\mathrm{~m}, 3 \mathrm{H}, \mathrm{NHC}\left(\mathrm{NH}_{2}\right)=\mathrm{N}\right], 6.92(\mathrm{~s}$, 1H, 1-H), 7.24 (t, 1H, J=7.5 Hz, Ind), 7.30 (t, 1H, $J=7.5 \mathrm{~Hz}$, Ind), 7.51 (s, 1H, Ind), 7.64 (d, 
$1 \mathrm{H}, J=7.5 \mathrm{~Hz}$, Ind), $8.12(\mathrm{~d}, 1 \mathrm{H}, J=7.5 \mathrm{~Hz}, \mathrm{Ind}) ;{ }^{13} \mathrm{C} \mathrm{NMR}\left(100 \mathrm{MHz},\left(\mathrm{CD}_{3}\right)_{2} \mathrm{CO}\right) \delta(\mathrm{ppm})$ : 12.5, 18.2, $19.5\left[3 \mathrm{CH}_{3}(\mathrm{Pbf})\right], 27.4\left(\mathrm{CH}_{2}\right.$-Ind), $27.8\left[\mathrm{C}_{\gamma}(\mathrm{Arg})\right], 28.3\left[3 \mathrm{CH}_{3}(\mathrm{Boc})\right], 28.5\left[\mathrm{C}_{\beta}\right.$ (Arg) and $\left.3 \mathrm{CH}_{3}\left({ }^{\mathrm{t}} \mathrm{Bu}\right)\right], 28.7\left[3 \mathrm{CH}_{3}(\mathrm{Boc})\right.$ and $\left.2 \mathrm{CH}_{3}(\mathrm{Pbf})\right], 41.6\left[\mathrm{C}_{\delta}(\mathrm{Arg})\right], 41.8\left(\mathrm{C}_{6}\right), 43.6$ $\left[\mathrm{CH}_{2}(\mathrm{Pbf})\right], 51.7\left(\mathrm{C}_{5}-\mathrm{CH}\right), 51.9\left(4-\mathrm{CH}_{2}\right), 55.6\left(\mathrm{C}_{5}\right), 64.9\left(\mathrm{C}_{3}\right), 78.8$ [C (Boc)], $81.6\left[\mathrm{C}\left({ }^{\mathrm{t}} \mathrm{Bu}\right)\right]$, 83.9 [C (Boc)], 86.9 [C (Pbf)], 115.8 [CH (Ind)], 117.4 [C (Pbf)], 118.9 [C (Ind)], 120.0, 123.1, 124.5, 124.9 [4CH (Ind)], 125.3 [C (Pbf)], 131.9 [C (Ind)], 132.8, 135.6 [2C (Pbf)],

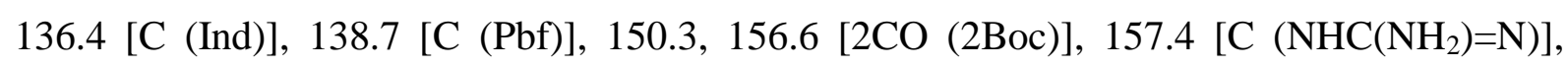
158.9 [C (Pbf)], $171.0\left(\mathrm{CO}_{2}\right), 171.8\left(\mathrm{C}_{2}\right)$; ES-MS m/z 925.2 [M+1] $]^{+}$; Anal. calcd. for $\mathrm{C}_{47} \mathrm{H}_{69} \mathrm{~N}_{7} \mathrm{O}_{10} \mathrm{~S}: \mathrm{C}, 61.08 ; \mathrm{H}, 7.53 ; \mathrm{N}, 10.61$. Found: C, 61.35; H, 7.29; N, 10.48 .

4.5.8. (5S,3S)-5-((S)-1-((tert-Butoxycarbonyl)amino-2-(1-(tert-butoxycarbonyl)-indol-3-

yl)ethyl-4-(tert-butoxycarbonyl)methyl -3-(3-(2-((2,2,4,6,7-pentamethyl-2,3dihydrobenzofuran-5-yl)sulfonyl)guanidino)propyl)-2-oxopiperazine [(S)-12a]

Reddish solid (305 mg, 50\%); $[\alpha]_{\mathrm{D}}{ }^{20}+9.0$ (c $\left.1.2, \mathrm{CH}_{2} \mathrm{Cl}_{2}\right) ; \mathrm{Mp}: 120-122{ }^{\circ} \mathrm{C}$ (EtOAc/hexane); HPLC [Sunfire $\mathrm{C}_{18}(4.6 \times 150 \mathrm{~mm}, 3.5 \mu \mathrm{m}), 80-100 \%$ gradient of solvent A in $\mathrm{B}, 30 \mathrm{~min}] t_{\mathrm{R}} 7.19 \mathrm{~min} ;{ }^{1} \mathrm{H}$ NMR (400 MHz, $\left.\left(\mathrm{CD}_{3}\right)_{2} \mathrm{CO}\right) \delta(\mathrm{ppm}): 1.32$ (s, 9H, Boc), 1.43 [s, $6 \mathrm{H}, 2 \mathrm{CH}_{3}$ (Pbf)], 1.44 (s, 9H, $\left.{ }^{\mathrm{B}} \mathrm{Bu}\right), 1.66$ [s, 3H, Boc (Ind)], 1.77 [m, 4H, $\beta-\mathrm{H}$ and $\gamma-\mathrm{H}$ (Arg)], 2.03 [s, 3H, $\left.\mathrm{CH}_{3}(\mathrm{Pbf})\right], 2.50$ [s, 3H, $\left.\mathrm{CH}_{3}(\mathrm{Pbf})\right], 2.57$ [s, 3H, $\left.\mathrm{CH}_{3}(\mathrm{Pbf})\right], 2.79$ (dd, 1H, J = 10 and $14 \mathrm{~Hz}, \mathrm{CH}_{2}$-Ind), 2.96 [s, 2H, $\mathrm{CH}_{2}$ (Pbf)], 3.20 (m, 1H, $\mathrm{CH}_{2}$-Ind), 3.21 (m, 1H, 5-H), 3.24 $[\mathrm{m}, 2 \mathrm{H}, \delta-\mathrm{H}(\mathrm{Arg})], 3.32[\mathrm{~m}, 1 \mathrm{H}, 3-\mathrm{H}], 3.46(\mathrm{~m}, 1 \mathrm{H}, 6-\mathrm{H}), 3.55\left(\mathrm{~m}, 2 \mathrm{H}, 4-\mathrm{CH}_{2}\right.$ and 6-H), 3.66 $\left(\mathrm{d}, 1 \mathrm{H}, J=18 \mathrm{~Hz}, 4-\mathrm{CH}_{2}\right), 4.05(\mathrm{~m}, 1 \mathrm{H}, 5-\mathrm{CH}), 6.24$ (d, 1H, J = 8.5 Hz, NHBoc), 6.50 [m, $\left.3 \mathrm{H}, \mathrm{NHC}\left(\mathrm{NH}_{2}\right)=\mathrm{N}\right], 7.15(\mathrm{~s}, 1 \mathrm{H}, 1-\mathrm{H}), 7.24(\mathrm{t}, 1 \mathrm{H}, J=7.5 \mathrm{~Hz}, \mathrm{Ind}), 7.31(\mathrm{t}, 1 \mathrm{H}, J=7.5 \mathrm{~Hz}$, Ind), 7.51 (s, 1H, Ind), 7.64 (d, $1 \mathrm{H}, J=7.5 \mathrm{~Hz}$, Ind), 8.12 (d, $1 \mathrm{H}, J=7.5 \mathrm{~Hz}$, Ind); ${ }^{13} \mathrm{C}$ NMR $\left(100 \mathrm{MHz},\left(\mathrm{CD}_{3}\right)_{2} \mathrm{CO}\right) \delta$ (ppm): 12.5, 18.2, $19.5\left[3 \mathrm{CH}_{3}(\mathrm{Pbf})\right], 25.5\left(\mathrm{CH}_{2}\right.$-Ind), $26.4\left[\mathrm{C}_{\gamma}\right.$ (Arg) $], 28.3\left[6 \mathrm{CH}_{3}\left(\mathrm{Boc}\right.\right.$ and $\left.\left.{ }^{\mathrm{t}} \mathrm{Bu}\right)\right], 28.6\left[2 \mathrm{CH}_{3}(\mathrm{Pbf})\right], 28.7$ [3 $\left.\mathrm{CH}_{3}(\mathrm{Boc})\right], 31.6\left[\mathrm{C}_{\beta}(\mathrm{Arg})\right]$, $39.9\left[\mathrm{C}_{6}\right], 41.6\left[\mathrm{C}_{\delta}(\mathrm{Arg})\right], 43.6\left[\mathrm{CH}_{2}(\mathrm{Pbf})\right], 52.1\left(\mathrm{C}_{5}-\mathrm{CH}\right), 56.9\left(4-\mathrm{CH}_{2}\right), 60.9\left(\mathrm{C}_{5}\right), 64.6\left(\mathrm{C}_{3}\right)$, 78.8 [C (Boc)], 81.7 [C ( $\left.\left.{ }^{\mathrm{t}} \mathrm{Bu}\right)\right], 84.0$ [C (Boc)], 86.9 [C (Pbf)], 115.9 [CH (Ind)], 117.4 [C (Pbf)], 119.1 [C (Ind)], 120.0, 123.1, 124.1, 125.0 [4CH (Ind)], 125.6 [C (Pbf)], 131.8 [C (Ind)], 132.8, 135.7 [2C (Pbf)], 136.4 [C (Ind)], 138.7 [C (Pbf)], 150.3, 156.5 [2CO (2Boc)], $157.3\left[\mathrm{C}\left(\mathrm{NHC}\left(\mathrm{NH}_{2}\right)=\mathrm{N}\right)\right], 158.9$ [C $\left.(\mathrm{Pbf})\right], 171.3\left(\mathrm{CO}_{2}\right.$ and $\left.\mathrm{C}_{2}\right)$; ES-MS $m / z 925.1[\mathrm{M}+1]^{+}$; Anal. calcd. for $\mathrm{C}_{47} \mathrm{H}_{69} \mathrm{~N}_{7} \mathrm{O}_{10} \mathrm{~S}$ : C, 61.08; H, 7.53; N, 10.61. Found: C, 61.29; H, 7.75; N, 10.40 . 
4.5.9. (5R,3S)-5-((S)-1-((tert-Butoxycarbonyl)amino-2-(1-(tert-butoxycarbonyl)-indol-3-

yl)ethyl-4-(tert-butoxycarbonyl)methyl -3-(4-((tert-butoxycarbonyl)amino)butyl)-2oxopiperazine [(R)-12b]

Reddish solid (368 mg, 75\%); $[\alpha]_{\mathrm{D}}{ }^{20}+3\left(c\right.$ 1.7, $\left.\mathrm{CH}_{2} \mathrm{Cl}_{2}\right) ; \mathrm{Mp}: 78-80{ }^{\circ} \mathrm{C}$ (EtOAc/hexane); HPLC [Sunfire $\mathrm{C}_{18}(4.6 \times 150 \mathrm{~mm}, 3.5 \mu \mathrm{m}), 70-100 \%$ gradient of solvent $\mathrm{A}$ in $\mathrm{b}, 30 \mathrm{~min}$ ] $t_{\mathrm{R}}$ $11.54 \mathrm{~min} ;{ }^{1} \mathrm{H}$ NMR (400 MHz, $\left.\left(\mathrm{CD}_{3}\right)_{2} \mathrm{CO}\right) \delta(\mathrm{ppm}): 1.29$ (s, 9H, Boc), 1.36 [s, 9H, Boc (Lys)], 1.49 (s, 9H, $\left.{ }^{\mathrm{t}} \mathrm{Bu}\right), 1.59$ [m, 2H, $\gamma$-H (Lys)], 1.60 [m, 2H, $\delta$-H (Lys)], 1.67 [s, 9H, Boc (Ind)], 1.79 [m, 1H, $\beta$-H (Lys)], 1.80 [m, 1H, $\beta$-H (Lys)], 2.78 (dd, 1H, $J=11$ and $15 \mathrm{~Hz}$, $\mathrm{CH}_{2}$-Ind), 3.12 [m, 2H, $\varepsilon-\mathrm{H}$ (Lys)], 3.19 (m, 2H, 3-H and 4- $\left.\mathrm{CH}_{2}\right), 3.39$ (m, 1H, 5-H), 3.41 (m, 2H, 6-H), $3.64\left(\mathrm{~d}, 1 \mathrm{H}, J=16 \mathrm{~Hz}, 4-\mathrm{CH}_{2}\right), 3.66$ (dd, $1 \mathrm{H}, J=2$ and $\left.15 \mathrm{~Hz}, \mathrm{CH}_{2}-\mathrm{Ind}\right), 3.94$ (m, 1H, 5-CH), 5.93 [m, 1H, NHBoc (Lys)], 6.07 (d, 1H, J = 9.5 Hz, NHBoc), 6.84 (s, 1H, 1-H), 7.23 (t, 1H, $J=7.5 \mathrm{~Hz}$, Ind), 7.30 (t, 1H, $J=7.5 \mathrm{~Hz}$, Ind), 7.51 (s, 1H, Ind), 7.64 (d, 1H, $J=$ $7.5 \mathrm{~Hz}, \mathrm{Ind}), 8.13\left(\mathrm{~d}, 1 \mathrm{H}, J=7.5 \mathrm{~Hz}\right.$, Ind). ${ }^{13} \mathrm{C} \mathrm{NMR}\left(100 \mathrm{MHz},\left(\mathrm{CD}_{3}\right)_{2} \mathrm{CO}\right) \delta$ (ppm): $24.7\left[\mathrm{C}_{\gamma}\right.$

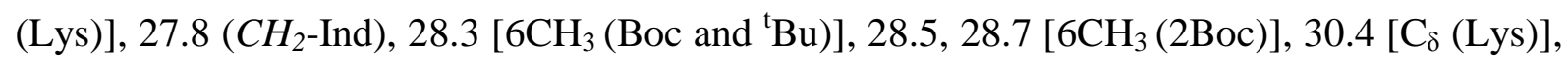
$31.1\left[\mathrm{C}_{\beta}(\mathrm{Lys})\right], 41.2\left[\mathrm{C}_{\varepsilon}\right.$ (Lys)], $41.8\left(\mathrm{C}_{6}\right), 51.9\left(\mathrm{C}_{5}-\mathrm{CH}\right), 52.1\left(4-\mathrm{CH}_{2}\right), 55.8\left(\mathrm{C}_{5}\right), 65.2\left(\mathrm{C}_{3}\right)$, 78.2, 78.8 [2C (2Boc)], 81.5[C ( $\left.{ }^{\mathrm{t}} \mathrm{Bu}\right)$ ], 83.9 [C (Boc)], 115.8 [CH (Ind)], 118.9 [C (Ind)], 120.0, 123.1, 124.3, 124.9 [4CH (Ind)], 132.0, 136.4 [2C (Ind)], 150.3, 156.6 [3CO (3Boc)], $171.0\left(\mathrm{CO}_{2}\right), 171.8\left(\mathrm{C}_{2}\right)$; ES-MS m/z $744.9[\mathrm{M}+1]^{+}$; Anal. calcd. for $\mathrm{C}_{39} \mathrm{H}_{61} \mathrm{~N}_{5} \mathrm{O}_{9}: \mathrm{C}, 62.97 ; \mathrm{H}$, 8.26; N, 9.41. Found: C, 63.21; H, 8.15; N, 9.24.

4.5.10. (5S,3S)-5-((S)-1-((tert-Butoxycarbonyl)amino-2-(1-(tert-butoxycarbonyl)-indol-3-

yl)ethyl-4-(tert-butoxycarbonyl)methyl -3-(4-((tert-butoxycarbonyl)amino)butyl)-2oxopiperazine [(S)-12b]

Reddish solid (295 mg, 60\%); $[\alpha]_{\mathrm{D}}{ }^{20}+10$ (c 1.2, $\mathrm{CH}_{2} \mathrm{Cl}_{2}$ ); $\mathrm{Mp}: 80-82{ }^{\circ} \mathrm{C}$ (EtOAc/hexane); HPLC [Sunfire $\mathrm{C}_{18}(4.6 \times 150 \mathrm{~mm}, 3.5 \mu \mathrm{m}), 70-100 \%$ gradient of solvent $\mathrm{A}$ in B, $30 \mathrm{~min}$ ] $t_{\mathrm{R}}$ $12.04 \mathrm{~min} ;{ }^{1} \mathrm{H}$ NMR (400 MHz, $\left.\left(\mathrm{CD}_{3}\right)_{2} \mathrm{CO}\right) \delta(\mathrm{ppm}): 1.34$ (s, 9H, Boc), 1.37 [s, 9H, Boc (Lys)], 1.45 (s, 9H, $\left.{ }^{\mathrm{t}} \mathrm{Bu}\right), 1.53$ [m, 2H, $\gamma$-H (Lys)], 1.56 [m, 2H, $\delta$-H (Lys)], 1.66 [s, 9H, Boc (Ind)], 1.77 [m, 2H, $\beta$-H (Lys)], 2.80 (m, 1H, $\mathrm{CH}_{2}$-Ind), 3.07 [m, 2H, $\varepsilon-\mathrm{H}$ (Lys)], 3.23 (m, 1H, $\mathrm{CH}_{2}$-Ind), 3.27 (m, 1H, 5-H), 3.30 (m, 1H, 3-H), 3.50 (m, 2H, 6-H), 3.57 (d, 1H, J=18 Hz, 4$\left.\mathrm{CH}_{2}\right), 3.68\left(\mathrm{~d}, 1 \mathrm{H}, J=18 \mathrm{~Hz}, 4-\mathrm{CH}_{2}\right), 4.07$ (m, 1H, 5-CH), 5.93 [m, 1H, NHBoc (Lys)], 6.21 (d, 1H, $J=8.5 \mathrm{~Hz}, N H \mathrm{Boc}), 7.00(\mathrm{~s}, 1 \mathrm{H}, 1-\mathrm{H}), 7.26$ (t, 1H, $J=7.5 \mathrm{~Hz}, \mathrm{Ind}), 7.31$ (t, 1H, $J=$ $7.5 \mathrm{~Hz}$, Ind), 7.52 (s, 1H, Ind), 7.67 (d, $1 \mathrm{H}, J=7.5 \mathrm{~Hz}$, Ind), 8.13 (d, $1 \mathrm{H}, J=7.5 \mathrm{~Hz}$, Ind). ${ }^{13} \mathrm{C}$ 
NMR (100 MHz, $\left.\left(\mathrm{CD}_{3}\right)_{2} \mathrm{CO}\right) \delta(\mathrm{ppm}): 23.8\left[\mathrm{C}_{\gamma}(\mathrm{Lys})\right], 25.6\left(\mathrm{CH}_{2}\right.$-Ind), $28.3\left[6 \mathrm{CH}_{3}(\mathrm{Boc}\right.$ and $\left.\left.{ }^{\mathrm{t}} \mathrm{Bu}\right)\right], 28.6\left[6 \mathrm{CH}_{3}(2 \mathrm{Boc})\right], 30.4\left[\mathrm{C}_{\delta}(\mathrm{Lys})\right], 34.5\left[\mathrm{C}_{\beta}(\mathrm{Lys})\right], 39.9\left(\mathrm{C}_{6}\right), 41.1\left[\mathrm{C}_{\varepsilon}(\mathrm{Lys})\right], 52.0$ $\left(\mathrm{C}_{5}-\mathrm{CH}\right), 57.0\left(4-\mathrm{CH}_{2}\right), 60.1\left(\mathrm{C}_{5}\right), 65.3\left(\mathrm{C}_{3}\right), 78.2,78.8$ [2C (2Boc)], $81.7\left[\mathrm{C}\left({ }^{\mathrm{t}} \mathrm{Bu}\right)\right], 84.0[\mathrm{C}$ (Boc)], 115.9 [CH (Ind)], 119.1 [C (Ind)], 119.6, 123.3, 124.1, 125.0 [4CH (Ind)], 131.8, 136.4 [2C (Ind)], 150.3, 156.4 [3CO (3Boc)], $171.3\left(\mathrm{CO}_{2}\right), 172.6\left(\mathrm{C}_{2}\right)$; ES-MS m/z 744.9 $[\mathrm{M}+1]^{+}$; Anal. calcd. for $\mathrm{C}_{39} \mathrm{H}_{61} \mathrm{~N}_{5} \mathrm{O}_{9}: \mathrm{C}, 62.97 ; \mathrm{H}, 8.26 ; \mathrm{N}, 9.41$. Found: $\mathrm{C}, 62.71 ; \mathrm{H}, 8.05 ; \mathrm{N}$, 9.63 .

4.5.11. (5R,3S)-5-((S)-1-(tert-Butoxycarbonyl)amino-2-phenylethyl)- 4-(tert-butoxycarbonyl)methyl -3-(3-(2-((2,2,4,6,7-pentamethyl-2,3-dihydrobenzofuran-5-yl)sulfonyl)guanidino) propyl)-2-oxopiperazine [(R)-13a]

Reddish solid (363 mg, 70\%); $[\alpha]_{\mathrm{D}}{ }^{20}-19$ (c $\left.1.0, \mathrm{CH}_{2} \mathrm{Cl}_{2}\right) ; \mathrm{Mp}: 100-102 \quad{ }^{\circ} \mathrm{C}$ (EtOAc/hexane); HPLC [Sunfire $\mathrm{C}_{18}(4.6 \times 150 \mathrm{~mm}, 3.5 \mu \mathrm{m}), 60-100 \%$ gradient of solvent A in $\mathrm{B}, 30 \mathrm{~min}] t_{\mathrm{R}} 15.95 \mathrm{~min} ;{ }^{1} \mathrm{H} \mathrm{NMR}\left(400 \mathrm{MHz},\left(\mathrm{CD}_{3}\right)_{2} \mathrm{CO}\right) \delta(\mathrm{ppm}): 1.26$ (s, 9H, Boc), 1.44 [s, 6H, 2CH $(\mathrm{Pbf})], 1.46\left(\mathrm{~s}, 9 \mathrm{H},{ }^{\mathrm{t}} \mathrm{Bu}\right), 1.72[\mathrm{~m}, 2 \mathrm{H}, \gamma-\mathrm{H}(\mathrm{Arg})], 1.95$ [m, 2H, $\left.\beta-\mathrm{H}(\mathrm{Arg})\right], 2.04$ [s, 3H, $\left.\mathrm{CH}_{3}(\mathrm{Pbf})\right], 2.50$ [s, 3H, $\left.\mathrm{CH}_{3}(\mathrm{Pbf})\right], 2.58$ [s, 3H, $\mathrm{CH}_{3}(\mathrm{Pbf})$ ], 2.67 (dd, 1H, $J=11$ and $\left.14 \mathrm{~Hz}, \mathrm{CH}_{2}-\mathrm{Ph}\right), 2.97$ [s, 2H, CH 2 (Pbf)], 3.15 (m, 2H, 3-H and 4- $\mathrm{CH}_{2}$ ), 3.30 [m, 2H, $\delta-\mathrm{H}$ (Arg)], $3.32(\mathrm{~m}, 1 \mathrm{H}, 5-\mathrm{H}), 3.39(\mathrm{~m}, 2 \mathrm{H}, 6-\mathrm{H}), 3.50\left(\mathrm{dd}, 1 \mathrm{H}, J=3\right.$ and $\left.14 \mathrm{~Hz}, \mathrm{CH}_{2}-\mathrm{Ph}\right), 3.61$ (d, $\left.1 \mathrm{H}, J=16 \mathrm{~Hz}, 4-\mathrm{CH}_{2}\right), 3.85(\mathrm{~m}, 1 \mathrm{H}, 5-\mathrm{CH}), 5.97$ (d, 1H, J = 9.5 Hz, NHBoc), 6.55 [m, $\left.3 \mathrm{H}, \mathrm{NHC}\left(\mathrm{NH}_{2}\right)=\mathrm{N}\right], 6.90(\mathrm{~s}, 1 \mathrm{H}, 1-\mathrm{H}), 7.13-7.19(\mathrm{~m}, 5 \mathrm{H}, \mathrm{Ph}) ;{ }^{13} \mathrm{C} \mathrm{NMR}(100 \mathrm{MHz}$, $\left.\left(\mathrm{CD}_{3}\right)_{2} \mathrm{CO}\right) \delta(\mathrm{ppm}): 12.5,18.2,19.5\left[3 \mathrm{CH}_{3}(\mathrm{Pbf})\right], 27.3\left[\mathrm{C}_{\gamma}(\mathrm{Arg})\right], 28.3\left[3 \mathrm{CH}_{3}\left({ }^{\mathrm{t}} \mathrm{Bu}\right)\right], 28.5$ [3CH $\left.3 \mathrm{CH}_{3}(\mathrm{Boc})\right], 28.7\left[\mathrm{C}_{\beta}(\mathrm{Arg})\right.$ and $\left.2 \mathrm{CH}_{3}(\mathrm{Pbf})\right], 38.2\left(\mathrm{CH}_{2}-\mathrm{Ph}\right), 41.6\left[\mathrm{C}_{\delta}(\mathrm{Arg})\right], 41.8\left[\mathrm{C}_{6}\right], 43.6$ [CH $2(\mathrm{Pbf})], 51.7\left(4-\mathrm{CH}_{2}\right), 54.0\left(\mathrm{C}_{5}-\mathrm{CH}\right), 55.5\left(\mathrm{C}_{5}\right), 64.9\left(\mathrm{C}_{3}\right), 78.7$ [C (Boc)], $81.4\left[\mathrm{C}\left({ }^{\mathrm{t}} \mathrm{Bu}\right)\right]$, 86.9, 117.4, 125.3 [3C (Pbf)], 126.7, 128.8, 130.3 [5CH (Ph)], 132.8, 135.6, 138.7 [3C (Pbf)], $140.5[\mathrm{C}(\mathrm{Ph})], 156.4[\mathrm{CO}(\mathrm{Boc})], 157.4\left[\mathrm{C}\left(\mathrm{NHC}\left(\mathrm{NH}_{2}\right)=\mathrm{N}\right)\right], 158.9[\mathrm{C}(\mathrm{Pbf})], 171.0\left(\mathrm{CO}_{2}\right)$, $171.8\left(\mathrm{C}_{2}\right)$; ES-MS m/z $785.7[\mathrm{M}+1]^{+}$; Anal. calcd. for $\mathrm{C}_{40} \mathrm{H}_{60} \mathrm{~N}_{6} \mathrm{O}_{8} \mathrm{~S}: \mathrm{C}, 61.20 ; \mathrm{H}, 7.70 ; \mathrm{N}$, 10.71. Found: C, 61.48; H, 7.40; N, 11.00.

4.5.12. (5S,3S)-5-((S)-1-(tert-Butoxycarbonyl)amino-2-phenylethyl)- 4-(tert-butoxycarbonyl)methyl-3-(3-(2-((2,2,4,6,7-pentamethyl-2,3-dihydrobenzofuran-5-yl)sulfonyl)guanidino) propyl)-2-oxopiperazine [(S)-13a]

Reddish solid (363 mg, 70\%); $[\alpha]_{\mathrm{D}}{ }^{20}+11\left(\right.$ c 1.2, $\left.\mathrm{CH}_{2} \mathrm{Cl}_{2}\right)$; Mp: 98-100 ${ }^{\circ} \mathrm{C}$ (EtOAc/hexane); HPLC [Sunfire $\mathrm{C}_{18}(4.6 \times 150 \mathrm{~mm}, 3.5 \mu \mathrm{m}), 60-100 \%$ gradient of solvent A in B, $30 \mathrm{~min}$ ] $t_{\mathrm{R}}$ 
$14.98 \mathrm{~min} ;{ }^{1} \mathrm{H}$ NMR (400 MHz, $\left.\left(\mathrm{CD}_{3}\right)_{2} \mathrm{CO}\right) \delta(\mathrm{ppm}): 1.29$ (s, 9H, Boc), $1.44\left[\mathrm{~s}, 6 \mathrm{H}, 2 \mathrm{CH}_{3}\right.$ (Pbf)], 1.45 (s, 9H, $\left.{ }^{\mathrm{t}} \mathrm{Bu}\right), 1.70$ [m, 2H, $\gamma-\mathrm{H}$ (Arg)], 1.72 [m, 2H, $\left.\beta-\mathrm{H}(\mathrm{Arg})\right], 2.05$ [s, 3H, $\mathrm{CH}_{3}$ (Pbf)], 2.50 [s, 3H, $\mathrm{CH}_{3}$ (Pbf)], 2.58 [s, 3H, $\left.\mathrm{CH}_{3}(\mathrm{Pbf})\right], 2.63$ (dd, $1 \mathrm{H}, J=11$ and $14 \mathrm{~Hz}, \mathrm{CH}_{2^{-}}$ $\mathrm{Ph}), 2.98$ [s, 2H, $\left.\mathrm{CH}_{2}(\mathrm{Pbf})\right], 3.10\left(\mathrm{dd}, 1 \mathrm{H}, J=3\right.$ and $\left.14 \mathrm{~Hz}, \mathrm{CH}_{2}-\mathrm{Ph}\right), 3.19$ (m, 1H, 5-H), 3.24 [m, 2H, $\delta-\mathrm{H}(\mathrm{Arg})], 3.31[\mathrm{~m}, 1 \mathrm{H}, 3-\mathrm{H}], 3.38(\mathrm{~m}, 1 \mathrm{H}, 6-\mathrm{H}), 3.50(\mathrm{~m}, 1 \mathrm{H}, 6-\mathrm{H}), 3.51(\mathrm{~m}, 1 \mathrm{H}, 4-$ $\left.\mathrm{CH}_{2}\right), 3.65$ (d, $\left.1 \mathrm{H}, J=18 \mathrm{~Hz}, 4-\mathrm{CH}_{2}\right), 3.92(\mathrm{~m}, 1 \mathrm{H}, 5-\mathrm{CH}), 6.16(\mathrm{~d}, 1 \mathrm{H}, J=8.5 \mathrm{~Hz}, N H$ Boc $)$, $6.55\left[\mathrm{~m}, 3 \mathrm{H}, \mathrm{NHC}\left(\mathrm{NH}_{2}\right)=\mathrm{N}\right], 7.10-7.21(\mathrm{~m}, 2 \mathrm{H}, 1-\mathrm{H}$ and $\mathrm{Ph}), 7.22-7.31(\mathrm{~m}, 4 \mathrm{H}, \mathrm{Ph}) ;{ }^{13} \mathrm{C}$

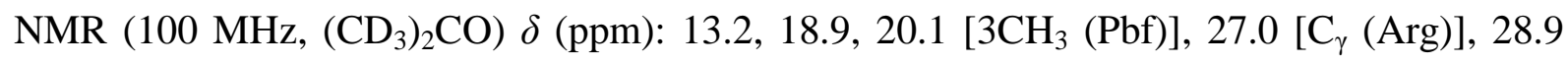
$\left[3 \mathrm{CH}_{3}\left({ }^{\mathrm{t}} \mathrm{Bu}\right)\right], 29.0\left[3 \mathrm{CH}_{3}(\mathrm{Boc})\right], 29.2\left[2 \mathrm{CH}_{3}(\mathrm{Pbf})\right], 32.3\left[\mathrm{C}_{\beta}(\mathrm{Arg})\right], 36.4\left(\mathrm{CH}_{2}-\mathrm{Ph}\right), 40.6$ $\left(\mathrm{C}_{6}\right), 42.3\left[\mathrm{C}_{\delta}(\mathrm{Arg})\right], 44.3\left[\mathrm{CH}_{2}(\mathrm{Pbf})\right], 54.8\left(\mathrm{C}_{5}-\mathrm{CH}\right), 57.4\left(4-\mathrm{CH}_{2}\right), 61.4\left(\mathrm{C}_{5}\right), 65.3\left(\mathrm{C}_{3}\right), 79.4$ [C (Boc)], 82.4 [C ( $\left.\left.{ }^{\mathrm{t}} \mathrm{Bu}\right)\right], 87.6,118.1,125.9$ [3C (Pbf)], 127.5, 129.7, 130.6 [5CH (Ph)], 133.5, 136.3, 139.4 [3C (Pbf)], 141.3 [C (Ph)], 156.9 [CO $(\mathrm{Boc})], 158.0$ [C $\left.\left(\mathrm{NHC}\left(\mathrm{NH}_{2}\right)=\mathrm{N}\right)\right]$, $159.5[\mathrm{C}(\mathrm{Pbf})], 171.2\left(\mathrm{CO}_{2}\right), 173.5\left(\mathrm{C}_{2}\right)$; ES-MS m/z $785.7[\mathrm{M}+1]^{+}$; Anal. calcd. for $\mathrm{C}_{40} \mathrm{H}_{60} \mathrm{~N}_{6} \mathrm{O}_{8} \mathrm{~S}: \mathrm{C}, 61.20 ; \mathrm{H}, 7.70 ; \mathrm{N}, 10.71$. Found: C, 61.01; H, 7.62; N, 10.94.

4.6. General procedure for the benzylation of the 4-alkyl-2-oxopiperazines 10a-13a. Synthesis of the 4-alkyl-1-benzyl-2-oxopiperazines (R)- and (S)-(14a, 17a, 20a and 23a), and (R)- and (S)-(16a, 19a, 22a and 25a)

$\mathrm{NaH}(60 \%$ suspension in mineral oil, $24 \mathrm{mg}, 1.02 \mathrm{mmol})$ and benzyl bromide $(152 \mu \mathrm{L}$, $0.51 \mathrm{mmol}$ ) were added in three equal portions over $2 \mathrm{~h}$ to a solution of the corresponding 2oxopiperazine 10a-13a $(0.34 \mathrm{mmol})$ in anhydrous mixture THF/DMF $(9: 1,10 \mathrm{~mL})$ under argon at $0{ }^{\circ} \mathrm{C}$. After $1 \mathrm{~h}$ of stirring, the crude reaction mixture was diluted with EtOAc (100 $\mathrm{mL})$ and the excess of $\mathrm{NaH}$ was hydrolysed by addition of $\mathrm{H}_{2} \mathrm{O}(20 \mathrm{~mL})$. The aqueous layer was extracted with EtOAc $(2 \times 50 \mathrm{~mL})$ and the organic extracts were dried over $\mathrm{Na}_{2} \mathrm{SO}_{4}$ and evaporated to dryness. The residue was purified by flash chromatography, using $\mathrm{MeOH}$ in $\mathrm{CH}_{2} \mathrm{Cl}_{2}$ gradient as mobile phase to give the respective 4-alkyl-1-benzyl-2-oxopiperazines 14a, 17a, 20a and 23a in 40-57\% and the respective derivative benzylated at the guanidino group 16a, 19a, 22a and 25a as (Z/E)-isomeric mixtures in 11-20\% yield. The compounds were dissolved in $\mathrm{CH}_{3} \mathrm{CN} / \mathrm{H}_{2} \mathrm{O}(1: 2,2 \mathrm{~mL})$ and the solutions were lyophilized to obtain amorphous solids. 
4.6.1. (5R,3S)-1,4-Dibenzyl-5-((S)-1-((tert-butoxycarbonyl)amino-2-(1-(tert-butoxycarbonyl)-indol-3-yl)ethyl-3-(3-(2-((2,2,4,6,7-pentamethyl-2,3-dihydrobenzofuran-5yl)sulfonyl)guanidino)propyl)-2-oxopiperazine [(R)-14a]

Amorphous solid (135 mg, 40\%); $[\alpha]_{\mathrm{D}}^{20}-12\left(c\right.$ 1.2, $\left.\mathrm{CH}_{2} \mathrm{Cl}_{2}\right)$; HPLC [Sunfire $\mathrm{C}_{18}(4.6 \times 150$ $\mathrm{mm}, 3.5 \mu \mathrm{m}), 80-100 \%$ gradient of solvent $\mathrm{A}$ in $\mathrm{B}, 30 \mathrm{~min}] t_{\mathrm{R}} 13.05 \mathrm{~min} ;{ }^{1} \mathrm{H} \mathrm{NMR}(400 \mathrm{MHz}$, $\left.\left(\mathrm{CD}_{3}\right)_{2} \mathrm{CO}\right) \delta$ (ppm): 1.24 (s, 9H, Boc), 1.42 [s, 6H, 2CH 3 (Pbf)], 1.64 [s, 3H, Boc (Ind)], 1.85 [m, 4H, $\beta-\mathrm{H}$ and $\gamma-\mathrm{H}(\mathrm{Arg})], 2.03\left[\mathrm{~s}, 3 \mathrm{H}, \mathrm{CH}_{3}(\mathrm{Pbf})\right], 2.49$ [s, 3H, $\left.\mathrm{CH}_{3}(\mathrm{Pbf})\right], 2.56[\mathrm{~s}, 3 \mathrm{H}$, $\mathrm{CH}_{3}$ (Pbf)], 2.83 (m, 1H, $\mathrm{CH}_{2}$-Ind), 2.92 [s, 2H, $\mathrm{CH}_{2}$ (Pbf)], 3.09 [m, 2H, $\delta$-H (Arg)], 3.16 [m, 1H, 3-H], 3.39 (m, 1H, 6-H), 3.53 [m, 1H, $\left.\mathrm{CH}_{2}-\mathrm{Ind}\right], 3.54$ [m, 1H, 4-CH $\left.\mathrm{CH}_{2}(\mathrm{Bn})\right], 3.58$ (m, 2H, 5-H and 6-H), $4.14[\mathrm{~m}, 1 \mathrm{H}, 5-\mathrm{CH}], 4.17\left[\mathrm{~d}, 1 \mathrm{H}, J=13.5 \mathrm{~Hz}, 4-\mathrm{CH}_{2}(\mathrm{Bn})\right], 4.51[\mathrm{~d}, 1 \mathrm{H}, J=$ $\left.14.5 \mathrm{~Hz}, 1-\mathrm{CH}_{2}(\mathrm{Bn})\right], 4.79\left[\mathrm{~d}, 1 \mathrm{H}, J=14.5 \mathrm{~Hz}, 1-\mathrm{CH}_{2}(\mathrm{Bn})\right], 5.98(\mathrm{~d}, 1 \mathrm{H}, J=9.5 \mathrm{~Hz}$, $N H$ Boc $), 6.47\left[\mathrm{~m}, 3 \mathrm{H}, \mathrm{NHC}\left(\mathrm{NH}_{2}\right)=\mathrm{N}\right], 7.07-7.55(\mathrm{~m}, 14 \mathrm{H}, \mathrm{Ar}), 8.10(\mathrm{~d}, 1 \mathrm{H}, J=8 \mathrm{~Hz}, \mathrm{Ar})$. ${ }^{13} \mathrm{C}$ NMR (100 MHz, $\left.\left(\mathrm{CD}_{3}\right)_{2} \mathrm{CO}\right) \delta(\mathrm{ppm}): 12.6,18.2,19.5$ [3CH $\left.3(\mathrm{Pbf})\right], 27.2\left(\mathrm{CH}_{2}\right.$-Ind), 27.9 [C $\left.\mathrm{C}_{\gamma}(\mathrm{Arg})\right], 28.3\left[3 \mathrm{CH}_{3}(\mathrm{Boc})\right], 28.5\left[2 \mathrm{CH}_{3}(\mathrm{Pbf})\right], 28.6\left[3 \mathrm{CH}_{3}(\mathrm{Boc})\right], 28.8\left[\mathrm{C}_{\beta}(\mathrm{Arg})\right], 41.3$ $\left[\mathrm{C}_{\delta}(\mathrm{Arg})\right], 43.6\left[\mathrm{CH}_{2}(\mathrm{Pbf})\right], 45.6\left(\mathrm{C}_{6}\right), 50.2$ [1- $\left.\mathrm{CH}_{2}(\mathrm{Bn})\right], 51.3\left(\mathrm{C}_{5}-\mathrm{CH}\right), 51.9$ [4- $\left.\mathrm{CH}_{2}(\mathrm{Bn})\right]$, $55.7\left(\mathrm{C}_{5}\right), 62.2\left(\mathrm{C}_{3}\right), 78.9,83.9$ [2C (2Boc)], 86.9 [C (Pbf)], 115.8 [CH (Ar)], 117.4 [C (Pbf)], 118.7 [C (Ar)], 119.9, 123.2, 124.4, 124.9 [4CH (Ar)], 125.3 [C (Pbf)], 128.2, 128.8, 129.3, 129.5, 129.8 [10CH (Ar)], 131.9 [C (Ar)], 132.8, 135.6 [2C (Pbf)], 136.3 [C (Ar)], 138.7 [2C (Pbf and Ar)], 139.4 [C (Ar)], 150.3, 156.5 [2CO (2Boc)], 157.3 [C $\left.\left.\left(\mathrm{NHC}^{\mathrm{N}} \mathrm{NH}_{2}\right)=\mathrm{N}\right)\right], 158.9$ [C (Pbf)], $170.8\left(\mathrm{C}_{2}\right)$; ES-MS m/z $991.1[\mathrm{M}+1]^{+}$; Anal. calcd. for $\mathrm{C}_{55} \mathrm{H}_{71} \mathrm{~N}_{7} \mathrm{O}_{8} \mathrm{~S}: \mathrm{C}, 66.71 ; \mathrm{H}$, 7.23; N, 9.90. Found: C, 66.98; H, 7.41; N, 9.54.

4.6.2. (5S,3S)-1,4-Dibenzyl-5-((S)-1-((tert-butoxycarbonyl)amino-2-(1-(tert-butoxycarbonyl)-indol-3-yl)ethyl-3-(3-(2-((2,2,4,6,7-pentamethyl-2,3-dihydrobenzofuran-5yl)sulfonyl)guanidino)propyl)-2-oxopiperazine [(S)-14a]

Amorphous solid (175 mg, 52\%); $[\alpha]_{\mathrm{D}}{ }^{20}+22\left(\mathrm{c} 1.1, \mathrm{CH}_{2} \mathrm{Cl}_{2}\right)$; HPLC [Sunfire $\mathrm{C}_{18}(4.6 \times$ $150 \mathrm{~mm}, 3.5 \mu \mathrm{m}), 80-100 \%$ gradient of solvent $\mathrm{A}$ in $\mathrm{B}, 30 \mathrm{~min}] t_{\mathrm{R}} 13.87 \mathrm{~min} ;{ }^{1} \mathrm{H}$ NMR (400 $\left.\mathrm{MHz},\left(\mathrm{CD}_{3}\right)_{2} \mathrm{CO}\right) \delta(\mathrm{ppm}): 1.29$ (s, 9H, Boc), 1.42 [s, 6H, 2CH $\left.(\mathrm{Pbf})\right], 1.66$ [s, 3H, Boc (Ind)], 1.73 [m, 4H, $\beta-\mathrm{H}$ and $\gamma-\mathrm{H}(\mathrm{Arg})], 2.03$ [s, 3H, $\left.\mathrm{CH}_{3}(\mathrm{Pbf})\right], 2.48$ [s, 3H, $\mathrm{CH}_{3}$ (Pbf)], 2.56 [s, 3H, $\mathrm{CH}_{3}$ (Pbf)], 2.78 (m, 1H, $\mathrm{CH}_{2}$-Ind), 2.94 [s, 2H, $\left.\mathrm{CH}_{2}(\mathrm{Pbf})\right], 3.00[\mathrm{~m}, 1 \mathrm{H}, \delta-\mathrm{H}$ (Arg)], 3.07 [m, 1H, $\delta$-H (Arg)], 3.13 (m, 1H, $C_{2}$-Ind), 3.15 (m, 1H, 5-H), 3.26 [m, 1H, 3$\mathrm{H}], 3.53(\mathrm{~m}, 2 \mathrm{H}, 6-\mathrm{H}), 3.81\left[\mathrm{~d}, 1 \mathrm{H}, J=14 \mathrm{~Hz}, 4-\mathrm{CH}_{2}(\mathrm{Bn})\right], 4.04\left[\mathrm{~d}, 1 \mathrm{H}, J=14 \mathrm{~Hz}, 4-\mathrm{CH}_{2}\right.$ 
(Bn)], $4.10(\mathrm{~m}, 1 \mathrm{H}, 5-\mathrm{CH}), 4.53\left[\mathrm{~d}, 1 \mathrm{H}, J=15 \mathrm{~Hz}, 1-\mathrm{CH}_{2}(\mathrm{Bn})\right], 4.69[\mathrm{~d}, 1 \mathrm{H}, J=15 \mathrm{~Hz}, 1-$ $\left.\mathrm{CH}_{2}(\mathrm{Bn})\right], 6.13(\mathrm{~d}, 1 \mathrm{H}, J=9 \mathrm{~Hz}, N H \mathrm{Boc}), 6.39$ [m, 3H, NHC( $\left.\left.\mathrm{NH}_{2}\right)=\mathrm{N}\right], 7.14-7.36(\mathrm{~m}, 11 \mathrm{H}$, Ar), 7.39-7.50 (m, 2H, Ar), 7.58 (d, $1 \mathrm{H}, J=7.5 \mathrm{~Hz}, \mathrm{Ar}), 8.12$ (d, $1 \mathrm{H}, J=7.5 \mathrm{~Hz}, \mathrm{Ar}) .{ }^{13} \mathrm{C}$

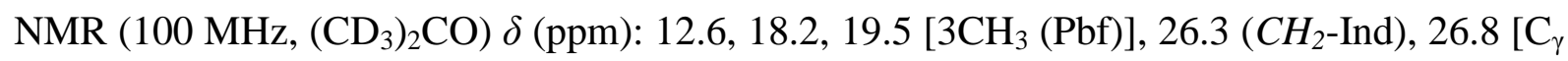
(Arg) $, 28.3\left[3 \mathrm{CH}_{3}(\mathrm{Boc})\right], 28.6\left[2 \mathrm{CH}_{3}(\mathrm{Pbf})\right], 28.7$ [3 $\left.\mathrm{CH}_{3}(\mathrm{Boc})\right], 31.9\left[\mathrm{C}_{\beta}(\mathrm{Arg})\right], 41.3\left[\mathrm{C}_{\delta}\right.$ (Arg) ], $43.6\left[\mathrm{CH}_{2}(\mathrm{Pbf})\right], 45.2\left(\mathrm{C}_{6}\right), 49.9$ [1- $\left.\mathrm{CH}_{2}(\mathrm{Bn})\right], 53.2\left(\mathrm{C}_{5}-\mathrm{CH}\right), 62.0$ [4- $\left.\mathrm{CH}_{2}(\mathrm{Bn})\right], 63.2$ $\left(\mathrm{C}_{5}\right), 63.7\left(\mathrm{C}_{3}\right), 79.0,84.0$ [2C (2Boc)], 86.9 [C (Pbf)], 115.9 [CH (Ar)], 117.4 [C (Pbf)], 118.8 [C (Ar)], 120.0, 123.2, 124.1, 125.0 [4CH (Ar)], 125.2 [C (Pbf)], 128.2, 128.7, 129.1, 129.5, 130.4 [10CH (Ar)], 131.9 [C (Ar)], 132.8, 135.7 [2C (Pbf)], 136.3 [C (Ar)], 138.6 [C

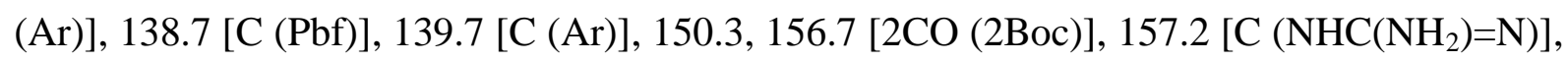
158.9 [C (Pbf)], $171.8\left(\mathrm{C}_{2}\right)$; ES-MS m/z $991.2[\mathrm{M}+1]^{+}$; Anal. calcd. for $\mathrm{C}_{55} \mathrm{H}_{71} \mathrm{~N}_{7} \mathrm{O}_{8} \mathrm{~S}: \mathrm{C}$, 66.71; H, 7.23; N, 9.90. Found: C, 66.85; H, 7.52; N, 9.61.

4.6.3. (5R,3S)-1,4-Dibenzyl-5-((S)-1-((tert-butoxycarbonyl)amino-2-(1-(tert-butoxycarbonyl)-indol-3-yl)ethyl-3-(3-(3-benzyl-2-((2,2,4,6,7-pentamethyl-2,3dihydrobenzofuran-5-yl)sulfonyl)guanidino)propyl)-2-oxopiperazine [(R)-16a]

Amorphous solid (2:1 isomeric mixture, $55 \mathrm{mg}, 15 \%)$; HPLC [Sunfire $\mathrm{C}_{18}(4.6 \times 150 \mathrm{~mm}$, $3.5 \mu \mathrm{m}), 80-100 \%$ gradient of solvent $\mathrm{A}$ in $\mathrm{B}, 30 \mathrm{~min}] t_{\mathrm{R}} 20.20 \mathrm{~min} ;{ }^{1} \mathrm{H}$ NMR (400 MHz, $\left.\left(\mathrm{CD}_{3}\right)_{2} \mathrm{CO}\right) \delta(\mathrm{ppm})$ Major isomer: $1.25(\mathrm{~s}, 9 \mathrm{H}, \mathrm{Boc}), 1.40$ [s, 3H, $\left.\mathrm{CH}_{3}(\mathrm{Pbf})\right], 1.45$ [s, 3H, $\mathrm{CH}_{3}$ (Pbf)], 1.63 [s, 3H, Boc (Ind)], 1.83 [m, 2H, $\gamma-\mathrm{H}$ (Arg)], 1.92 [m, 2H, $\beta-\mathrm{H}$ (Arg)], 2.00 [s, 3H, $\mathrm{CH}_{3}$ (Pbf)], 2.45 [s, 3H, $\mathrm{CH}_{3}$ (Pbf)], 2.50 [s, 3H, $\mathrm{CH}_{3}$ (Pbf)], 2.81 [s, 2H, $\mathrm{CH}_{2}$ (Pbf)], $2.90\left(\mathrm{~m}, 1 \mathrm{H}, \mathrm{CH}_{2}\right.$-Ind), 3.07 [m, 2H, $\delta$-H (Arg)], 3.14 [m, 1H, 3-H], 3.38 (m, 1H, 6-H), 3.55 [m, 2H, 5-H and 4- $\left.\mathrm{CH}_{2}(\mathrm{Bn})\right], 3.57(\mathrm{~m}, 1 \mathrm{H}, 6-\mathrm{H}), 3.58\left[\mathrm{~m}, 1 \mathrm{H}, \mathrm{CH}_{2}-\mathrm{Ind}\right], 4.20$ [m, 1H, 4- $\mathrm{CH}_{2}$ $(\mathrm{Bn})], 4.22[\mathrm{~m}, 1 \mathrm{H}, 5-\mathrm{CH}], 4.43\left[\mathrm{t}, 2 \mathrm{H}, J=5.5 \mathrm{~Hz}, \mathrm{CH}_{2}(\mathrm{NHC}(\mathrm{NHBn})=\mathrm{N})\right], 4.54[\mathrm{~d}, 1 \mathrm{H}, J=$ $\left.15 \mathrm{~Hz}, 1-\mathrm{CH}_{2}(\mathrm{Bn})\right], 4.78$ [d, 1H, $\left.J=15 \mathrm{~Hz}, 1-\mathrm{CH}_{2}(\mathrm{Bn})\right], 5.99$ (d, 1H, $J=9.5 \mathrm{~Hz}, N H$ Boc), 7.02-7.57 [m, 21H, Ar and $N H C(N H B n)=\mathrm{N}], 8.11(\mathrm{~d}, 1 \mathrm{H}, J=8 \mathrm{~Hz}, \mathrm{Ar})$. Minor isomer: 1.22 (s, 9H, Boc), 1.38 [s, 3H, $\mathrm{CH}_{3}$ (Pbf)], 1.45 [s, 3H, $\mathrm{CH}_{3}$ (Pbf)], 1.63 [s, 3H, Boc (Ind)], 1.83 [m, 2H, $\gamma-\mathrm{H}(\mathrm{Arg})], 1.92$ [m, 2H, $\beta$-H (Arg)], 1.99 [s, 3H, $\left.\mathrm{CH}_{3}(\mathrm{Pbf})\right], 2.45$ [s, 3H, CH 3 (Pbf)], 2.50 [s, 3H, $\mathrm{CH}_{3}$ (Pbf)], 2.83 (m, 1H, $\mathrm{CH}_{2}$-Ind), 2.93 [s, 2H, $\mathrm{CH}_{2}$ (Pbf)], 3.07 [m, 2H, $\delta-\mathrm{H}$ (Arg)], 3.14 [m, 1H, 3-H], 3.38 (m, 1H, 6-H), 3.47 [m, 1H, $\mathrm{CH}_{2}$-Ind], 3.55 [m, 2H, 5-H and 4$\left.\mathrm{CH}_{2}(\mathrm{Bn})\right], 3.57$ (m, 1H, 6-H), 4.20 [m, 1H, 4- $\left.\mathrm{CH}_{2}(\mathrm{Bn})\right], 4.22$ [m, 1H, 5-CH], 4.48 [d, 1H, J $\left.=15 \mathrm{~Hz}, 1-\mathrm{CH}_{2}(\mathrm{Bn})\right], 4.64\left[\mathrm{~m}, 2 \mathrm{H}, \mathrm{CH}_{2}(\mathrm{NHC}(\mathrm{NHBn})=\mathrm{N})\right], 4.81\left[\mathrm{~d}, 1 \mathrm{H}, J=15 \mathrm{~Hz}, 1-\mathrm{CH}_{2}\right.$ $(\mathrm{Bn})], 5.94(\mathrm{~d}, 1 \mathrm{H}, J=9.5 \mathrm{~Hz}, N H \mathrm{Boc}), 7.02-7.57[\mathrm{~m}, 21 \mathrm{H}, \mathrm{Ar}$ and $N H \mathrm{C}(N H \mathrm{Bn})=\mathrm{N}], 8.11(\mathrm{~d}$, 
$1 \mathrm{H}, J=8 \mathrm{~Hz}, \mathrm{Ar}) ;{ }^{13} \mathrm{C}$ NMR $\left(100 \mathrm{MHz},\left(\mathrm{CD}_{3}\right)_{2} \mathrm{CO}\right) \delta(\mathrm{ppm})$ Major isomer: 12.5, 18.3, 19.6 [3 $\left.3 \mathrm{CH}_{3}(\mathrm{Pbf})\right], 25.6\left(\mathrm{CH}_{2}\right.$-Ind), $27.0\left[\mathrm{C}_{\gamma}(\mathrm{Arg})\right], 28.0\left[\mathrm{C}_{\beta}(\mathrm{Arg})\right], 28.3\left[3 \mathrm{CH}_{3}(\mathrm{Boc})\right.$ and $2 \mathrm{CH}_{3}$ $(\mathrm{Pbf})], 28.6\left[3 \mathrm{CH}_{3}(\mathrm{Boc})\right], 41.6\left[\mathrm{C}_{\delta}(\mathrm{Arg})\right], 43.6\left[\mathrm{CH}_{2}(\mathrm{Pbf})\right], 45.3\left[\mathrm{CH}_{2}(\mathrm{NHC}(\mathrm{NHBn})=\mathrm{N})\right]$, $45.9\left(\mathrm{C}_{6}\right), 50.3\left[1-\mathrm{CH}_{2}(\mathrm{Bn})\right], 51.4\left(\mathrm{C}_{5}-\mathrm{CH}\right), 51.8\left[4-\mathrm{CH}_{2}(\mathrm{Bn})\right], 55.7\left(\mathrm{C}_{5}\right), 61.9\left(\mathrm{C}_{3}\right), 78.8$, 83.9 [2C (2Boc)], 86.9 [C (Pbf)], 115.8 [CH (Ar)], 117.6 [C (Pbf)], 118.7 [C (Ar)], 120.0, 123.2, 124.5, 124.9 [4CH (Ar)], 125.3 [C (Pbf)], 128.2, 128.8, 129.4, 129.5, 129.8 [15CH (Ar)], 131.9 [C (Ar)], 132.8, 135.4 [2C (Pbf)], 136.3 [C (Ar)], 138.7 [2C (Pbf and Ar)], 138.8 [C (Ar)], 139.3 [C (Ar)], 150.3, 156.0 [2CO (2Boc)], 156.5 [C (NHC(NHBn)=N)], 158.9 [C (Pbf)], $170.7\left(\mathrm{C}_{2}\right)$. Minor isomer: 12.5, 18.3, 19.5 [3 $\left.\mathrm{CH}_{3}(\mathrm{Pbf})\right], 25.6\left[\mathrm{CH}_{2}\right.$-Ind $], 27.0\left[\mathrm{C}_{\gamma}\right.$ (Arg) $, 28.0\left[\mathrm{C}_{\beta}(\mathrm{Arg})\right], 28.3\left[3 \mathrm{CH}_{3}(\mathrm{Boc})\right.$ and $\left.2 \mathrm{CH}_{3}(\mathrm{Pbf})\right], 28.6\left[3 \mathrm{CH}_{3}(\mathrm{Boc})\right], 41.6\left[\mathrm{C}_{\delta}\right.$ (Arg) $], 43.6\left[\mathrm{CH}_{2}(\mathrm{Pbf})\right], 45.9\left(\mathrm{C}_{6}\right), 50.3\left[1-\mathrm{CH}_{2}(\mathrm{Bn})\right], 51.5\left(\mathrm{C}_{5}-\mathrm{CH}\right), 51.8\left[4-\mathrm{CH}_{2}(\mathrm{Bn})\right.$ and $\left.\mathrm{CH}_{2}(\mathrm{NHC}(\mathrm{NHBn})=\mathrm{N})\right], 55.6\left(\mathrm{C}_{5}\right), 62.0\left(\mathrm{C}_{3}\right), 78.8,83.9$ [2C (2Boc)], 86.9 [C $\left.(\mathrm{Pbf})\right], 115.8$ [CH (Ar)], 117.5 [C (Pbf)], 118.6 [C (Ar)], 119.9, 123.2, 124.5, 124.9 [4CH (Ar)], 125.3 [C (Pbf)], 128.2, 128.9, 129.2, 129.4, 129.5 [15CH (Ar)], 131.9 [C (Ar)], 132.8, 135.4 [2C (Pbf)], 136.3 [C (Ar)], 138.7 [2C (Pbf and Ar)], 138.8 [C (Ar)], 139.3 [C (Ar)], 150.3, 156.0 [2CO (2Boc)], 156.5 [C (NHC(NHBn)=N)], 158.9 [C (Pbf)], $170.7\left(\mathrm{C}_{2}\right)$; ES-MS m/z 1081.2 $[\mathrm{M}+1]^{+}$; Anal. calcd. for $\mathrm{C}_{62} \mathrm{H}_{77} \mathrm{~N}_{7} \mathrm{O}_{8} \mathrm{~S}: \mathrm{C}, 68.93$; H, 7.18; N, 9.08. Found: C, 69.24; H, 7.32; N, 8.91 .

4.6.4. (5S,3S)-1,4-Dibenzyl-5-((S)-1-((tert-butoxycarbonyl)amino-2-(1-(tert-butoxycarbonyl)-indol-3-yl)ethyl-3-(3-(3-benzyl-2-((2,2,4,6,7-pentamethyl-2,3dihydrobenzofuran-5-yl)sulfonyl)guanidino)propyl)-2-oxopiperazine [(S)-16a]

Amorphous solid (3:1 isomeric mixture, $55 \mathrm{mg}, 15 \%)$; HPLC [Sunfire $\mathrm{C}_{18}(4.6 \times 150 \mathrm{~mm}$, $3.5 \mu \mathrm{m}$ ), 80-100\% gradient of solvent $\mathrm{A}$ in $\mathrm{B}, 30 \mathrm{~min}] t_{\mathrm{R}} 20.78 \mathrm{~min}$ (major isomer) and 21.64 min (minor isomer); ${ }^{1} \mathrm{H}$ NMR $\left(400 \mathrm{MHz},\left(\mathrm{CD}_{3}\right)_{2} \mathrm{CO}\right) \delta$ (ppm) Major isomer: 1.29 (s, 9H, Boc), 1.42 [s, 3H, $\mathrm{CH}_{3}$ (Pbf)], 1.50 [s, 3H, $\mathrm{CH}_{3}$ (Pbf)], 1.64 [m, 2H, $\left.\gamma-\mathrm{H}(\mathrm{Arg})\right], 1.66$ [s, 3H, Boc (Ind)], 1.71 [m, 2H, $\beta-\mathrm{H}(\mathrm{Arg})], 2.01$ [s, 3H, $\mathrm{CH}_{3}$ (Pbf)], 2.42 [s, 3H, $\left.\mathrm{CH}_{3}(\mathrm{Pbf})\right], 2.49$ [s, $\left.3 \mathrm{H}, \mathrm{CH}_{3}(\mathrm{Pbf})\right], 2.79$ (m, 1H, $\left.\mathrm{CH}_{2}-\mathrm{Ind}\right), 2.88$ [s, 2H, $\left.\mathrm{CH}_{2}(\mathrm{Pbf})\right], 3.11$ [m, 2H, $\delta-\mathrm{H}$ (Arg)], $3.14[\mathrm{~m}, 2 \mathrm{H}, 5-\mathrm{H}], 3.15$ [m, 1H, $\mathrm{CH}_{2}$-Ind], 3.30 [m, 1H, 3-H], 3.53 (m, 2H, 6-H), 3.82 [d, 1H, $\left.J=14 \mathrm{~Hz}, 4-\mathrm{CH}_{2}(\mathrm{Bn})\right], 4.07\left[\mathrm{~m}, 1 \mathrm{H}, 4-\mathrm{CH}_{2}(\mathrm{Bn})\right], 4.13[\mathrm{~m}, 1 \mathrm{H}, 5-\mathrm{CH}], 4.38[\mathrm{~d}, 2 \mathrm{H}, J=5$ $\left.\mathrm{Hz}, \mathrm{CH}_{2}(\mathrm{NHC}(\mathrm{NHBn})=\mathrm{N})\right], 4.53\left[\mathrm{~m}, 1 \mathrm{H}, 1-\mathrm{CH}_{2}(\mathrm{Bn})\right], 4.69\left[\mathrm{~m}, 1 \mathrm{H}, 1-\mathrm{CH}_{2}(\mathrm{Bn})\right], 6.12(\mathrm{~d}$, $1 \mathrm{H}, J=8.5 \mathrm{~Hz}, N H \mathrm{Boc}), 7.02-7.37[\mathrm{~m}, 16 \mathrm{H}, \mathrm{Ar}$ and $N H \mathrm{C}(N H \mathrm{Bn})=\mathrm{N}], 7.44(\mathrm{~m}, 4 \mathrm{H}, \mathrm{Ar}), 7.60$ (m, $1 \mathrm{H}, \mathrm{Ar}), 8.12$ (d, 1H, $J=8 \mathrm{~Hz}, \mathrm{Ar}$ ). Minor isomer: 1.29 (s, 9H, Boc), 1.43 [s, 3H, $\mathrm{CH}_{3}$ 
(Pbf)], 1.45 [s, 3H, $\mathrm{CH}_{3}$ (Pbf)], 1.64 [m, 5H, $\gamma-\mathrm{H}$ (Arg) and Boc (Ind)], $1.71[\mathrm{~m}, 2 \mathrm{H}, \beta-\mathrm{H}$ (Arg)], 2.02 [s, 3H, $\mathrm{CH}_{3}$ (Pbf)], 2.46 [s, 3H, $\mathrm{CH}_{3}$ (Pbf)], 2.54 [s, 3H, $\mathrm{CH}_{3}$ (Pbf)], 2.79 (m, 1H, $\mathrm{CH}_{2}$-Ind), 2.97 [s, 2H, CH $\left.(\mathrm{Pbf})\right], 3.11[\mathrm{~m}, 2 \mathrm{H}, \delta-\mathrm{H}(\mathrm{Arg})], 3.14$ [m, 2H, 5-H], 3.15 [m, 1H, $\mathrm{CH}_{2}$-Ind], 3.27 [m, 1H, 3-H], $3.53(\mathrm{~m}, 2 \mathrm{H}, 6-\mathrm{H}), 3.81$ [d, 1H, $\left.J=14 \mathrm{~Hz}, 4-\mathrm{CH}_{2}(\mathrm{Bn})\right], 4.04$ $\left[\mathrm{m}, 1 \mathrm{H}, 4-\mathrm{CH}_{2}(\mathrm{Bn})\right], 4.13[\mathrm{~m}, 1 \mathrm{H}, 5-\mathrm{CH}], 4.59\left[\mathrm{~m}, 1 \mathrm{H}, \mathrm{CH}_{2}(\mathrm{NHC}(\mathrm{NHBn})=\mathrm{N})\right], 4.50[\mathrm{~m}, 1 \mathrm{H}$, $\left.1-\mathrm{CH}_{2}(\mathrm{Bn})\right], 4.60\left[\mathrm{~m}, 1 \mathrm{H}, \mathrm{CH}_{2}(\mathrm{NHC}(\mathrm{NHBn})=\mathrm{N})\right], 4.63\left[\mathrm{~m}, 1 \mathrm{H}, 1-\mathrm{CH}_{2}(\mathrm{Bn})\right], 6.07(\mathrm{~d}, 1 \mathrm{H}, J$ $=8.5 \mathrm{~Hz}, N H \mathrm{Boc}), 7.02-7.37[\mathrm{~m}, 16 \mathrm{H}, \mathrm{Ar}$ and $N H \mathrm{C}(N H \mathrm{Bn})=\mathrm{N}], 7.43(\mathrm{~m}, 4 \mathrm{H}, \mathrm{Ar}), 7.56(\mathrm{~m}$, $1 \mathrm{H}, \mathrm{Ar}), 8.12$ (d, $1 \mathrm{H}, J=8 \mathrm{~Hz}, \mathrm{Ar}) ;{ }^{13} \mathrm{C} \mathrm{NMR}\left(100 \mathrm{MHz},\left(\mathrm{CD}_{3}\right)_{2} \mathrm{CO}\right) \delta(\mathrm{ppm})$ Major isomer: 12.6, 18.3, $19.6\left[3 \mathrm{CH}_{3}(\mathrm{Pbf})\right], 26.2\left(\mathrm{CH}_{2}\right.$-Ind), $26.4\left[\mathrm{C}_{\gamma}(\mathrm{Arg})\right], 28.3\left[3 \mathrm{CH}_{3}(\mathrm{Boc})\right], 28.6\left[2 \mathrm{CH}_{3}\right.$ (Pbf)], $28.7\left[3 \mathrm{CH}_{3}(\mathrm{Boc})\right], 30.1\left[\mathrm{C}_{\beta}(\mathrm{Arg})\right], 41.6\left[\mathrm{C}_{\delta}(\mathrm{Arg})\right], 43.6\left[\mathrm{CH}_{2}(\mathrm{Pbf})\right], 44.9\left(\mathrm{C}_{6}\right), 45.3$ $\left[\mathrm{CH}_{2}(\mathrm{NHC}(\mathrm{NHBn})=\mathrm{N})\right], 49.9\left[1-\mathrm{CH}_{2}(\mathrm{Bn})\right], 52.7\left(\mathrm{C}_{5}-\mathrm{CH}\right), 61.9\left[4-\mathrm{CH}_{2}(\mathrm{Bn})\right], 63.5\left(\mathrm{C}_{3}\right.$ and $\left.\mathrm{C}_{5}\right), 78.9,84.0$ [2C (2Boc)], 86.9 [C (Pbf)], 115.9 [CH (Ar)], 117.5 [C (Pbf)], 118.9 [C (Ar)], 120.0, 123.2, 124.0, 125.0 [4CH (Ar)], 125.3 [C (Pbf)], 128.1, 128.7, 129.1, 129.5, 130.3 [15CH (Ar)], 131.8 [C (Ar)], 132.8, 135.9 [2C (Pbf)], 136.3 [C (Ar)], 138.4 [C (Ar)], 138.7 [C (Pbf], 139.6 [2C (Ar)], 150.3, 155.9 [2CO (2Boc)], 156.5 [C (NHC(NHBn)=N)], 158.9 [C (Pbf)], $171.7\left(\mathrm{C}_{2}\right)$. Minor isomer: 12.6, 18.2, 19.4 [3 $\left.\mathrm{CH}_{3}(\mathrm{Pbf})\right], 26.2\left(\mathrm{CH}_{2}-\mathrm{Ind}\right), 26.4\left[\mathrm{C}_{\gamma}\right.$ (Arg)], $28.1\left[3 \mathrm{CH}_{3}(\mathrm{Boc})\right], 28.6\left[2 \mathrm{CH}_{3}(\mathrm{Pbf})\right], 28.7\left[3 \mathrm{CH}_{3}(\mathrm{Boc})\right], 30.2\left[\mathrm{C}_{\beta}(\mathrm{Arg})\right], 41.6\left[\mathrm{C}_{\delta}\right.$ (Arg)], $43.6\left[\mathrm{CH}_{2}(\mathrm{Pbf})\right], 44.9\left(\mathrm{C}_{6}\right), 49.9\left[1-\mathrm{CH}_{2}(\mathrm{Bn})\right], 51.0\left[\mathrm{CH}_{2}(\mathrm{NHC}(\mathrm{NHBn})=\mathrm{N})\right], 52.7$ $\left(\mathrm{C}_{5}-\mathrm{CH}\right), 61.9$ [4- $\left.\mathrm{CH}_{2}(\mathrm{Bn})\right], 63.5\left(\mathrm{C}_{5}\right), 64.0\left(\mathrm{C}_{3}\right), 78.9,84.0$ [2C (2Boc)], 87.3 [C (Pbf)], 115.9 [CH (Ar)], 117.5 [C (Pbf)], 118.9 [C (Ar)], 120.0, 123.2, 124.0, 125.0 [4CH (Ar)], 125.3 [C (Pbf)], 128.1, 128.7, 129.2, 129.4, 130.3 [15CH (Ar)], 131.8 [C (Ar)], 132.8, 136.1 [2C (Pbf)], 136.3 [C (Ar)], 138.4 [C (Ar)], 138.7 [C (Pbf], 139.6 [2C (Ar)], 150.3, 155.9 [2CO (2Boc)], 156.5 [C (NHC(NHBn)=N)], 158.9 [C (Pbf)], $171.7\left(\mathrm{C}_{2}\right)$; ES-MS m/z 1081.2 $[\mathrm{M}+1]^{+}$; Anal. calcd. for $\mathrm{C}_{62} \mathrm{H}_{77} \mathrm{~N}_{7} \mathrm{O}_{8} \mathrm{~S}$ : C, 68.93; H, 7.18; N, 9.08. Found: C, 69.14; H, 7.02; N, 9.27.

4.6.5. (5R,3S)-1,4-Dibenzyl-5-((S)-1-(tert-butoxycarbonyl)amino-2-phenylethyl)-3-(3-(2((2,2,4,6,7-pentamethyl-2,3-dihydrobenzofuran-5-yl)sulfonyl)guanidino) propyl)-2oxopiperazine [(R)-17a]

Amorphous solid (139 mg, 48\%); $[\alpha]_{\mathrm{D}}{ }^{20}-12\left(c\right.$ 1.0, $\left.\mathrm{CH}_{2} \mathrm{Cl}_{2}\right)$; HPLC [Sunfire $\mathrm{C}_{18}(4.6 \times 150$ $\mathrm{mm}, 3.5 \mu \mathrm{m}), 80-100 \%$ gradient of solvent $\mathrm{A}$ in B, $30 \mathrm{~min}] t_{\mathrm{R}} 7.14 \mathrm{~min} ;{ }^{1} \mathrm{H}$ NMR $(400 \mathrm{MHz}$, $\left.\left(\mathrm{CD}_{3}\right)_{2} \mathrm{CO}\right) \delta(\mathrm{ppm}): 1.22$ (s, 9H, Boc), 1.43 [s, 6H, 2CH $\left.3(\mathrm{Pbf})\right], 1.81[\mathrm{~m}, 4 \mathrm{H}, \beta-\mathrm{H}$ and $\gamma-\mathrm{H}$ (Arg)], 2.04 [s, 3H, $\mathrm{CH}_{3}$ (Pbf)], 2.48 [s, 3H, $\left.\mathrm{CH}_{3}(\mathrm{Pbf})\right], 2.57$ [s, 3H, $\left.\mathrm{CH}_{3}(\mathrm{Pbf})\right], 2.71$ (dd, 1H, 
$J=11$ and $\left.14 \mathrm{~Hz}, \mathrm{CH}_{2}-\mathrm{Ph}\right), 2.96\left[\mathrm{~s}, 2 \mathrm{H}, \mathrm{CH}_{2}(\mathrm{Pbf})\right], 3.05$ [m, 2H, $\left.\delta-\mathrm{H}(\mathrm{Arg})\right], 3.12$ [m, 1H, 3$\mathrm{H}], 3.32(\mathrm{dd}, 1 \mathrm{H}, J=4$ and $12 \mathrm{~Hz}, 6-\mathrm{H}), 3.48\left(\mathrm{~m}, 2 \mathrm{H}, 5-\mathrm{H}\right.$ and $\left.\mathrm{CH}_{2}-\mathrm{Ph}\right), 3.50$ [m, $1 \mathrm{H}, 4-\mathrm{CH}_{2}$ (Bn)], 3.57 (m, 1H, 6-H), $4.06(\mathrm{ddd}, 1 \mathrm{H}, J=3.5,10$ and $13 \mathrm{~Hz}, 5-\mathrm{CH}), 4.17$ [d, 1H, $J=14.5$ $\left.\mathrm{Hz}, 4-\mathrm{CH}_{2}(\mathrm{Bn})\right], 4.47$ [d, $\left.1 \mathrm{H}, J=14.5 \mathrm{~Hz}, 1-\mathrm{CH}_{2}(\mathrm{Bn})\right], 4.78$ [d, $1 \mathrm{H}, J=14.5 \mathrm{~Hz}, 1-\mathrm{CH}_{2}$ (Bn)], $5.90(\mathrm{~d}, 1 \mathrm{H}, J=9.5 \mathrm{~Hz}, N H \mathrm{Boc}), 6.50$ [m, 3H, NHC( $\left.\left.\mathrm{NH}_{2}\right)=\mathrm{N}\right], 7.09-7.45(\mathrm{~m}, 15 \mathrm{H}$, $\mathrm{Ar}) ;{ }^{13} \mathrm{C}$ NMR (100 MHz, $\left.\left(\mathrm{CD}_{3}\right)_{2} \mathrm{CO}\right) \delta(\mathrm{ppm}): 11.9,17.6,18.8\left[3 \mathrm{CH}_{3}(\mathrm{Pbf})\right], 26.5\left[\mathrm{C}_{\gamma}(\mathrm{Arg})\right]$, $27.6\left[\mathrm{C}_{\beta}(\mathrm{Arg})\right], 27.8\left[3 \mathrm{CH}_{3}(\mathrm{Boc})\right], 28.1\left[2 \mathrm{CH}_{3}(\mathrm{Pbf})\right], 37.6\left(\mathrm{CH}_{2}-\mathrm{Ph}\right), 40.6\left[\mathrm{C}_{\delta}(\mathrm{Arg})\right], 42.9$ $\left[\mathrm{CH}_{2}(\mathrm{Pbf})\right], 45.0\left(\mathrm{C}_{6}\right), 49.5$ [1- $\left.\mathrm{CH}_{2}(\mathrm{Bn})\right], 51.0$ [4- $\left.\mathrm{CH}_{2}(\mathrm{Bn})\right], 52.4\left(\mathrm{C}_{5}-\mathrm{CH}\right), 55.0\left(\mathrm{C}_{5}\right), 61.5$ $\left(\mathrm{C}_{3}\right), 78.1$ [C (Boc)], 86.3, 116.8, 124.6 [3C (Pbf)], 126.1, 127.5, 128.1, 128.2, 128.6, 128.8, 129.3, 129.5 [15CH (Ar)], 132.2, 134.9 [2C (Pbf)], 138.0 [C (Ar)], 138.1 [2C (Pbf and Ar)], 139.5 [C (Ar)], 155.7 [CO (Boc)], $156.6\left[\mathrm{C}\left(\mathrm{NHC}\left(\mathrm{NH}_{2}\right)=\mathrm{N}\right)\right], 158.2[\mathrm{C}(\mathrm{Pbf})], 170.2\left(\mathrm{C}_{2}\right)$; ES-MS $m / z$ 852.0 [M+1] $]^{+}$; Anal. calcd. for $\mathrm{C}_{48} \mathrm{H}_{62} \mathrm{~N}_{6} \mathrm{O}_{6} \mathrm{~S}: \mathrm{C}, 67.74 ; \mathrm{H}, 7.34 ; \mathrm{N}, 9.87$. Found: C, 67.51; H, 7.65; N, 9.63.

4.6.6. (5S,3S)-1,4-Dibenzyl-5-((S)-1-(tert-butoxycarbonyl)amino-2-phenylethyl)-3-(3-(2((2,2,4,6,7-pentamethyl-2,3-dihydrobenzofuran-5-yl)sulfonyl)guanidino) propyl)-2oxopiperazine [(S)-17a]

Amorphous solid (130 mg, 45\%); $[\alpha]_{\mathrm{D}}{ }^{20}+21\left(c\right.$ 1.1, $\left.\mathrm{CH}_{2} \mathrm{Cl}_{2}\right)$; HPLC [Sunfire $\mathrm{C}_{18}(4.6 \times$ $150 \mathrm{~mm}, 3.5 \mu \mathrm{m}), 80-100 \%$ gradient of solvent $\mathrm{A}$ in $\mathrm{B}, 30 \mathrm{~min}] t_{\mathrm{R}} 6.96 \mathrm{~min} ;{ }^{1} \mathrm{H}$ NMR (400 $\left.\mathrm{MHz},\left(\mathrm{CD}_{3}\right)_{2} \mathrm{CO}\right) \delta(\mathrm{ppm}): 1.26(\mathrm{~s}, 9 \mathrm{H}, \mathrm{Boc}), 1.43$ [s, 6H, 2CH $\left.3(\mathrm{Pbf})\right], 1.56[\mathrm{~m}, 2 \mathrm{H}, \gamma-\mathrm{H}$ (Arg)], 1.67 [m, 2H, $\beta-\mathrm{H}(\mathrm{Arg})], 2.04$ [s, 3H, $\left.\mathrm{CH}_{3}(\mathrm{Pbf})\right], 2.49$ [s, 3H, $\left.\mathrm{CH}_{3}(\mathrm{Pbf})\right], 2.56$ [s, 3H, $\left.\mathrm{CH}_{3}(\mathrm{Pbf})\right], 2.60\left(\mathrm{~m}, 1 \mathrm{H}, \mathrm{CH}_{2}-\mathrm{Ph}\right), 2.96$ [s, 2H, $\left.\mathrm{CH}_{2}(\mathrm{Pbf})\right], 2.99$ [m, 1H, $\left.\delta-\mathrm{H}(\mathrm{Arg})\right], 3.04$ (m, 1H, $\left.\mathrm{CH}_{2}-\mathrm{Ph}\right), 3.05$ [m, 1H, $\left.\delta-\mathrm{H}(\mathrm{Arg})\right], 3.07$ (m, 2H, 5-H), 3.23 [m, 1H, 3-H], 3.45 (m, 2H, 6H), $3.77\left[\mathrm{~m}, 1 \mathrm{H}, J=14 \mathrm{~Hz}, 4-\mathrm{CH}_{2}(\mathrm{Bn})\right], 3.97$ (m, 1H, 5-CH), 4.03 [d, 1H, $J=14 \mathrm{~Hz}, 4-\mathrm{CH}_{2}$ (Bn)], 4.49 [d, 1H, J = $\left.15 \mathrm{~Hz}, 1-\mathrm{CH}_{2}(\mathrm{Bn})\right], 4.69$ [d, 1H, $\left.J=15 \mathrm{~Hz}, 1-\mathrm{CH}_{2}(\mathrm{Bn})\right], 6.09$ (d, 1H, $J=9 \mathrm{~Hz}, \mathrm{NHBoc}), 6.41\left[\mathrm{~m}, 3 \mathrm{H}, \mathrm{NHC}\left(\mathrm{NH}_{2}\right)=\mathrm{N}\right], 7.11-7.29(\mathrm{~m}, 15 \mathrm{H}, \mathrm{Ar}) ;{ }^{13} \mathrm{C} \mathrm{NMR}(100$ $\left.\mathrm{MHz},\left(\mathrm{CD}_{3}\right)_{2} \mathrm{CO}\right) \delta(\mathrm{ppm}): 12.6,18.2,19.5\left[3 \mathrm{CH}_{3}(\mathrm{Pbf})\right], 26.6\left[\mathrm{C}_{\gamma}(\mathrm{Arg})\right], 28.6\left[3 \mathrm{CH}_{3}(\mathrm{Boc})\right]$, $28.7\left[2 \mathrm{CH}_{3}(\mathrm{Pbf})\right], 31.9\left[\mathrm{C}_{\beta}(\mathrm{Arg})\right], 36.5\left(\mathrm{CH}_{2} \mathrm{Ph}\right), 41.2\left[\mathrm{C}_{\delta}(\mathrm{Arg})\right], 43.6\left[\mathrm{CH}_{2}(\mathrm{Pbf})\right], 45.1$ $\left(\mathrm{C}_{6}\right), 49.8\left[1-\mathrm{CH}_{2}(\mathrm{Bn})\right], 55.5\left(\mathrm{C}_{5}-\mathrm{CH}\right), 62.0\left[4-\mathrm{CH}_{2}(\mathrm{Bn})\right], 63.4\left(\mathrm{C}_{5}\right), 63.8\left(\mathrm{C}_{3}\right), 78.9[\mathrm{C}$ (Boc)], 86.9, 117.4, 125.3 [3C (Pbf)], 126.8, 128.2, 128.7, 128.9, 129.2, 129.5, 130.0, 130.4 [15CH (Ar)], 132.8, 135.7 [2C (Pbf)], 138.5 [C (Ar)], 138.7 [C (Pbf)], 139.9 [C (Ar)], 140.3

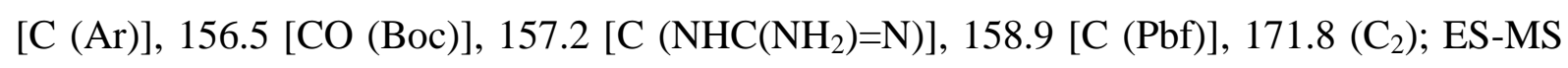


m/z 852.0 [M+1] $]^{+}$; Anal. calcd. for $\mathrm{C}_{48} \mathrm{H}_{62} \mathrm{~N}_{6} \mathrm{O}_{6} \mathrm{~S}: \mathrm{C}, 67.74 ; \mathrm{H}, 7.34 ; \mathrm{N}, 9.87$. Found: C, 67.86; $\mathrm{H}, 7.61 ; \mathrm{N}, 10.12$.

4.6.7. (5R,3S)-1,4-Dibenzyl-5-((S)-1-(tert-butoxycarbonyl)amino-2-phenylethyl)-3-(3-(3benzyl-2-((2,2,4,6,7-pentamethyl-2,3-dihydrobenzofuran-5-yl)sulfonyl)guanidino) propyl)-2-oxopiperazine [(R)-19a]

Amorphous solid (2.5:1 isomeric mixture, $45 \mathrm{mg}, 15 \%)$; HPLC [Sunfire $\mathrm{C}_{18}(4.6 \times 150$ $\mathrm{mm}, 3.5 \mu \mathrm{m}), 80-100 \%$ gradient of solvent $\mathrm{A}$ in $\mathrm{B}, 30 \mathrm{~min}] t_{\mathrm{R}} 13.14 \mathrm{~min} ;{ }^{1} \mathrm{H}$ NMR $(400 \mathrm{MHz}$, $\left.\left(\mathrm{CD}_{3}\right)_{2} \mathrm{CO}\right) \delta(\mathrm{ppm})$ Major isomer: 1.21 (s, 9H, Boc), 1.41 [s, 3H, $\left.\mathrm{CH}_{3}(\mathrm{Pbf})\right], 1.45$ [s, 3H, $\left.\mathrm{CH}_{3}(\mathrm{Pbf})\right], 1.80[\mathrm{~m}, 4 \mathrm{H}, \beta-\mathrm{H}$ and $\gamma-\mathrm{H}(\mathrm{Arg})], 2.01$ [s, 3H, $\left.\mathrm{CH}_{3}(\mathrm{Pbf})\right], 2.45$ [s, 3H, $\mathrm{CH}_{3}$ (Pbf)], 2.51 [s, 3H, $\mathrm{CH}_{3}$ (Pbf)], $2.74\left(\mathrm{dd}, 1 \mathrm{H}, J=11\right.$ and $\left.14 \mathrm{~Hz}, \mathrm{CH}_{2}-\mathrm{Ph}\right), 2.89$ [s, $2 \mathrm{H}, \mathrm{CH}_{2}$ (Pbf)], 3.05 [m, 2H, $\delta-\mathrm{H}(\mathrm{Arg})$ ], 3.15 [m, 1H, 3-H], 3.33 (dd, 1H, $J=4$ and $12 \mathrm{~Hz}, 6-\mathrm{H}), 3.50$ (m, 1H, 5-H), $3.52\left[\mathrm{~m}, 1 \mathrm{H}, 4-\mathrm{CH}_{2}(\mathrm{Bn})\right], 3.56\left(\mathrm{~m}, 1 \mathrm{H}, \mathrm{CH}_{2}-\mathrm{Ph}\right), 3.57(\mathrm{~m}, 1 \mathrm{H}, 6-\mathrm{H}), 4.12(\mathrm{~m}$, $1 \mathrm{H}, 5-\mathrm{CH}), 4.18$ [d, 1H, $\left.J=13.5 \mathrm{~Hz}, 4-\mathrm{CH}_{2}(\mathrm{Bn})\right], 4.42$ [m, 2H, $\left.\mathrm{CH}_{2}(\mathrm{NHC}(\mathrm{NHBn})=\mathrm{N})\right], 4.49$ $\left[\mathrm{d}, 1 \mathrm{H}, J=14.5 \mathrm{~Hz}, 1-\mathrm{CH}_{2}(\mathrm{Bn})\right], 4.79\left[\mathrm{~d}, 1 \mathrm{H}, J=14.5 \mathrm{~Hz}, 1-\mathrm{CH}_{2}(\mathrm{Bn})\right], 5.89$ (d, 1H, $J=9.5$ $\mathrm{Hz}, N H \mathrm{Boc})$, 7.04-7.57 [m, 22H, Ar and $N H \mathrm{C}(N H \mathrm{Bn})=\mathrm{N}]$. Minor isomer: $1.22(\mathrm{~s}, 9 \mathrm{H}$, Boc), 1.43 [s, 3H, $\mathrm{CH}_{3}$ (Pbf)], 1.44 [s, 3H, $\mathrm{CH}_{3}$ (Pbf)], 1.80 [m, 4H, $\beta-\mathrm{H}$ and $\left.\gamma-\mathrm{H}(\mathrm{Arg})\right], 2.01$ [s, 3H, $\left.\mathrm{CH}_{3}(\mathrm{Pbf})\right], 2.46$ [s, 3H, $\mathrm{CH}_{3}$ (Pbf)], 2.50 [s, 3H, $\left.\mathrm{CH}_{3}(\mathrm{Pbf})\right], 2.66$ (m, 1H, $\left.\mathrm{CH}_{2}-\mathrm{Ph}\right), 2.96$ [s, 2H, $\left.\mathrm{CH}_{2}(\mathrm{Pbf})\right], 3.05$ [m, 2H, $\left.\delta-\mathrm{H}(\mathrm{Arg})\right], 3.15$ [m, 1H, 3-H], 3.30 (m, 1H, 6-H), 3.41 (dd, $1 \mathrm{H}, J=3$ and $14 \mathrm{~Hz}, \mathrm{CH}_{2}-\mathrm{Ph}$ ), 3.50 (m, 1H, 5-H), 3.51 [m, 1H, 4- $\left.\mathrm{CH}_{2}(\mathrm{Bn})\right], 3.59$ (m, 1H, 6$\mathrm{H}), 4.10\left[\mathrm{~m}, 1 \mathrm{H}, 4-\mathrm{CH}_{2}(\mathrm{Bn})\right], 4.12(\mathrm{~m}, 1 \mathrm{H}, 5-\mathrm{CH}), 4.49$ [d, 1H, $\left.J=14.5 \mathrm{~Hz}, 1-\mathrm{CH}_{2}(\mathrm{Bn})\right]$, $4.60\left[\mathrm{~m}, 2 \mathrm{H}, \mathrm{CH}_{2}(\mathrm{NHC}(\mathrm{NHBn})=\mathrm{N})\right], 4.81\left[\mathrm{~d}, 1 \mathrm{H}, J=14.5 \mathrm{~Hz}, 1-\mathrm{CH}_{2}(\mathrm{Bn})\right], 5.86(\mathrm{~d}, 1 \mathrm{H}, J=$ $10 \mathrm{~Hz}, N H \mathrm{Boc}), 7.04-7.57[\mathrm{~m}, 22 \mathrm{H}, \mathrm{Ar}$ and $N H \mathrm{C}(N H \mathrm{Bn})=\mathrm{N}] ;{ }^{13} \mathrm{C} \mathrm{NMR}(100 \mathrm{MHz}$, $\left.\left(\mathrm{CD}_{3}\right)_{2} \mathrm{CO}\right) \delta(\mathrm{ppm})$ Major isomer: 11.9, 17.6, 18.9 [3 $\left.\mathrm{CH}_{3}(\mathrm{Pbf})\right], 26.2\left[\mathrm{C}_{\gamma}(\mathrm{Arg})\right], 27.6\left[\mathrm{C}_{\beta}\right.$ (Arg) and $\left.3 \mathrm{CH}_{3}(\mathrm{Boc})\right], 27.8$ [2 $\left.\mathrm{CH}_{3}(\mathrm{Pbf})\right], 37.6\left(\mathrm{CH}_{2}-\mathrm{Ph}\right), 40.5\left[\mathrm{C}_{\delta}(\mathrm{Arg})\right], 42.9\left[\mathrm{CH}_{2}(\mathrm{Pbf})\right]$, $45.1\left[\mathrm{C}_{6}\right.$ and $\left.\mathrm{CH}_{2}(\mathrm{NHC}(\mathrm{NHBn})=\mathrm{N})\right], 49.6\left[1-\mathrm{CH}_{2}(\mathrm{Bn})\right], 51.0\left[4-\mathrm{CH}_{2}(\mathrm{Bn})\right], 52.6\left(\mathrm{C}_{5}-\mathrm{CH}\right)$, 55.0 $\left(\mathrm{C}_{5}\right), 61.4\left(\mathrm{C}_{3}\right), 78.0$ [C (Boc)], 86.3, 116.9, 124.6 [3C (Pbf)], 127.5, 128.1, 128.3, 128.7, 128.8, 129.2, 129.6 [20CH (Ar)], 132.2, 134.9 [2C (Pbf)], 138.0 [C (Ar)], 138.1 [C (Ar)], 138.7 [C (Pbf)], 138.9 [C (Ar)], 139.5 [C (Ar)], 155.3 [C (NHC(NHBn)=N)], 155.6 [CO (Boc)], 158.3 [C (Pbf)], $170.1\left(\mathrm{C}_{2}\right)$. Minor isomer: 11.9, 17.6, 18.9 [3 $\left.\mathrm{CH}_{3}(\mathrm{Pbf})\right], 26.2\left[\mathrm{C}_{\gamma}\right.$

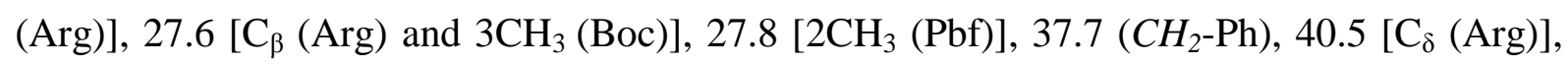
$42.9\left[\mathrm{CH}_{2}(\mathrm{Pbf})\right], 44.7\left(\mathrm{C}_{6}\right), 49.6\left[1-\mathrm{CH}_{2}(\mathrm{Bn})\right], 50.8\left[\mathrm{CH}_{2}(\mathrm{NHC}(\mathrm{NHBn})=\mathrm{N})\right], 51.0\left[4-\mathrm{CH}_{2}\right.$ $(\mathrm{Bn})$ ], $52.4\left(\mathrm{C}_{5}-\mathrm{CH}\right), 55.0\left(\mathrm{C}_{5}\right), 61.1\left(\mathrm{C}_{3}\right), 78.0$ [C (Boc)], 86.3, 116.9, 124.6 [3C (Pbf)], 
127.5, 128.2, 128.3, 128.5, 129.2, 129.5 [20CH (Ar)], 132.2, 134.9 [2C (Pbf)], 138.0 [C (Ar)], 138.1 [C (Ar)], 138.7 [C (Pbf)], 138.9 [C (Ar)], 139.5 [C (Ar)], 155.3 [C (NHC(NHBn)=N)], 155.7 [CO (Boc)], 158.3 [C (Pbf)], $170.1\left(\mathrm{C}_{2}\right)$; ES-MS m/z 942.2 [M+1] ${ }^{+}$; Anal. calcd. for $\mathrm{C}_{55} \mathrm{H}_{68} \mathrm{~N}_{6} \mathrm{O}_{6} \mathrm{~S}: \mathrm{C}, 70.18 ; \mathrm{H}, 7.28 ; \mathrm{N}, 8.93$. Found: C, 69.92; H, 7.47; N, 8.67.

4.6.8. (5S,3S)-1,4-Dibenzyl-5-((S)-1-(tert-butoxycarbonyl)amino-2-phenylethyl)-3-(3-(3benzyl-2-((2,2,4,6,7-pentamethyl-2,3-dihydrobenzofuran-5-yl)sulfonyl)guanidino) propyl)-2-oxopiperazine [(S)-19a]

Amorphous solid (5:3 isomeric mixture, $35 \mathrm{mg}, 11 \%)$; HPLC [Sunfire $\mathrm{C}_{18}(4.6 \times 150 \mathrm{~mm}$, $3.5 \mu \mathrm{m}$ ), 80-100\% gradient of solvent $\mathrm{A}$ in $\mathrm{B}, 30 \mathrm{~min}] t_{\mathrm{R}} 12.49$ (major isomer) and 13.19 (minor isomer) min; ${ }^{1} \mathrm{H}$ NMR $\left(400 \mathrm{MHz},\left(\mathrm{CD}_{3}\right)_{2} \mathrm{CO}\right) \delta(\mathrm{ppm})$ Major isomer: $1.26(\mathrm{~s}, 9 \mathrm{H}$, Boc), 1.44 [s, 6H, 2CH $(\mathrm{Pbf})], 1.64$ [m, 4H, $\beta-\mathrm{H}$ and $\gamma-\mathrm{H}(\mathrm{Arg})], 2.01$ [s, 3H, $\mathrm{CH}_{3}$ (Pbf)], $2.43\left[\mathrm{~s}, 3 \mathrm{H}, \mathrm{CH}_{3}(\mathrm{Pbf})\right], 2.50\left[\mathrm{~s}, 3 \mathrm{H}, \mathrm{CH}_{3}(\mathrm{Pbf})\right], 2.62\left(\mathrm{~m}, 1 \mathrm{H}, \mathrm{CH}_{2}-\mathrm{Ph}\right), 2.94\left[\mathrm{~s}, 2 \mathrm{H}, \mathrm{CH}_{2}\right.$ (Pbf)], 3.08 [m, 3H, 5-H and $\delta-\mathrm{H}(\mathrm{Arg})], 3.09$ (m, 1H, $\left.\mathrm{CH}_{2}-\mathrm{Ph}\right), 3.26$ [m, 1H, 3-H], 3.46 (m, 2H, 6-H), 3.78 [d, 1H, $\left.J=14 \mathrm{~Hz}, 4-\mathrm{CH}_{2}(\mathrm{Bn})\right], 4.06$ [d, 1H, $\left.J=14 \mathrm{~Hz}, 4-\mathrm{CH}_{2}(\mathrm{Bn})\right], 4.08$ (m, $1 \mathrm{H}, 5-\mathrm{CH}), 4.40\left[\mathrm{~d}, 2 \mathrm{H}, J=5.5 \mathrm{~Hz}, \mathrm{CH}_{2}(\mathrm{NHC}(\mathrm{NHBn})=\mathrm{N})\right], 4.49\left[\mathrm{~d}, 1 \mathrm{H}, J=15 \mathrm{~Hz}, 1-\mathrm{CH}_{2}\right.$ (Bn)], 4.68 [d, 1H, $\left.J=15 \mathrm{~Hz}, 1-\mathrm{CH}_{2}(\mathrm{Bn})\right], 6.05$ (d, 1H, $\left.J=8.5 \mathrm{~Hz}, N H \mathrm{Boc}\right), 7.02-7.52$ [m, $22 \mathrm{H}, \mathrm{Ar}$ and $N H \mathrm{C}(N H \mathrm{Bn})=\mathrm{N}]$. Minor isomer: $1.25(\mathrm{~s}, 9 \mathrm{H}, \mathrm{Boc}), 1.45\left[\mathrm{~s}, 6 \mathrm{H}, 2 \mathrm{CH}_{3}(\mathrm{Pbf})\right]$, $1.64[\mathrm{~m}, 4 \mathrm{H}, \beta-\mathrm{H}$ and $\gamma-\mathrm{H}(\mathrm{Arg})], 2.01\left[\mathrm{~s}, 3 \mathrm{H}, \mathrm{CH}_{3}(\mathrm{Pbf})\right], 2.47$ [s, 3H, $\left.\mathrm{CH}_{3}(\mathrm{Pbf})\right], 2.50[\mathrm{~s}$, $\left.3 \mathrm{H}, \mathrm{CH}_{3}(\mathrm{Pbf})\right], 2.62\left(\mathrm{~m}, 1 \mathrm{H}, \mathrm{CH}_{2}-\mathrm{Ph}\right), 2.97$ [s, 2H, $\left.\mathrm{CH}_{2}(\mathrm{Pbf})\right], 3.08$ [m, 3H, 5-H and $\delta-\mathrm{H}$ (Arg)], 3.09 (m, 1H, $\left.C_{2}-\mathrm{Ph}\right), 3.26[\mathrm{~m}, 1 \mathrm{H}, 3-\mathrm{H}], 3.46$ (m, 2H, 6-H), 3.77 [d, 1H, J = $14 \mathrm{~Hz}$, 4- $\left.\mathrm{CH}_{2}(\mathrm{Bn})\right], 4.06\left[\mathrm{~d}, 1 \mathrm{H}, J=14 \mathrm{~Hz}, 4-\mathrm{CH}_{2}(\mathrm{Bn})\right], 4.08$ (m, 1H, 5-CH), 4.49 [d, 1H, $J=15$ $\left.\mathrm{Hz}, 1-\mathrm{CH}_{2}(\mathrm{Bn})\right], 4.58\left[\mathrm{~m}, 2 \mathrm{H}, \mathrm{CH}_{2}(\mathrm{NHC}(\mathrm{NHBn})=\mathrm{N})\right], 4.64\left[\mathrm{~d}, 1 \mathrm{H}, J=15 \mathrm{~Hz}, 1-\mathrm{CH}_{2}(\mathrm{Bn})\right]$, 6.05 (d, $1 \mathrm{H}, J=8.5 \mathrm{~Hz}, N H \mathrm{Boc}), 7.02-7.52[\mathrm{~m}, 22 \mathrm{H}, \mathrm{Ar}$ and $N H \mathrm{C}(N H \mathrm{Bn})=\mathrm{N}] ;{ }^{13} \mathrm{C} \mathrm{NMR}$ $\left(100 \mathrm{MHz},\left(\mathrm{CD}_{3}\right)_{2} \mathrm{CO}\right) \delta(\mathrm{ppm})$ Major isomer: 12.5, 18.7, $19.6\left[3 \mathrm{CH}_{3}(\mathrm{Pbf})\right], 26.4\left[\mathrm{C}_{\gamma}(\mathrm{Arg})\right]$, $28.6\left[3 \mathrm{CH}_{3}\right.$ (Boc)], $29.3\left[2 \mathrm{CH}_{3}(\mathrm{Pbf})\right], 32.1\left[\mathrm{C}_{\beta}(\mathrm{Arg})\right], 36.3\left(\mathrm{CH}_{2}-\mathrm{Ph}\right), 41.6\left[\mathrm{C}_{\delta}(\mathrm{Arg})\right], 43.6$ $\left[\mathrm{CH}_{2}(\mathrm{Pbf})\right], 44.9\left[\mathrm{C}_{6}\right.$ and $\left.\mathrm{CH}_{2}(\mathrm{NHC}(\mathrm{NHBn})=\mathrm{N})\right], 49.8\left[1-\mathrm{CH}_{2}(\mathrm{Bn})\right], 54.7\left(\mathrm{C}_{5}-\mathrm{CH}\right), 61.7$ [4$\mathrm{CH}_{2}(\mathrm{Bn})$ ], $63.6\left(\mathrm{C}_{3}\right.$ and $\left.\mathrm{C}_{5}\right), 78.9$ [C (Boc)], 86.9, 117.4, 125.3 [3C (Pbf)], 126.8, 128.2, 128.7, 129.0, 129.1, 129.2, 129.5, 129.9, 130.3 [20CH (Ar)], 132.8, 135.6 [2C (Pbf)], 138.4 [C (Ar)], 138.7 [C (Pbf)], 139.5 [C (Ar)], 139.8 [C (Ar)], 139.9 [C (Ar)], 155.9 [C $(\mathrm{NHC}(\mathrm{NHBn})=\mathrm{N})], 156.3$ [CO $(\mathrm{Boc})], 158.9$ [C $(\mathrm{Pbf})], 171.8\left(\mathrm{C}_{2}\right)$. Minor isomer: 12.5, 18.7, $19.6\left[3 \mathrm{CH}_{3}(\mathrm{Pbf})\right], 26.4\left[\mathrm{C}_{\gamma}(\mathrm{Arg})\right], 28.6\left[3 \mathrm{CH}_{3}(\mathrm{Boc})\right], 29.3\left[2 \mathrm{CH}_{3}(\mathrm{Pbf})\right], 32.1\left[\mathrm{C}_{\beta}(\mathrm{Arg})\right]$, $36.3\left(\mathrm{CH}_{2}-\mathrm{Ph}\right), 41.6\left[\mathrm{C}_{\delta}(\mathrm{Arg})\right], 43.6\left[\mathrm{CH}_{2}(\mathrm{Pbf})\right], 44.9\left(\mathrm{C}_{6}\right), 49.9$ [1- $\left.\mathrm{CH}_{2}(\mathrm{Bn})\right], 51.0\left[\mathrm{CH}_{2}\right.$ 
$(\mathrm{NHC}(\mathrm{NHBn})=\mathrm{N})], 54.7\left(\mathrm{C}_{5}-\mathrm{CH}\right), 61.7\left[4-\mathrm{CH}_{2}(\mathrm{Bn})\right], 63.6\left(\mathrm{C}_{3}\right.$ and $\left.\mathrm{C}_{5}\right), 78.9[\mathrm{C}(\mathrm{Boc})], 86.9$, 117.4, 125.3 [3C (Pbf)], 126.8, 127.9, 128.2, 128.5, 128.8, 129.4, 129.9, 130.2 [20CH (Ar)], 132.8, 135.6 [2C (Pbf)], 138.4 [C (Ar)], 138.7 [C (Pbf)], 139.5 [C (Ar)], 139.8 [C (Ar)], 139.9 [C (Ar)], 156.5 [C (NHC(NHBn)=N)], 156.3 [CO (Boc)], 158.9 [C (Pbf)], $171.8\left(\mathrm{C}_{2}\right)$; ES-MS $m / z$ 942.2 [M+1] $]^{+}$; Anal. calcd. for $\mathrm{C}_{55} \mathrm{H}_{68} \mathrm{~N}_{6} \mathrm{O}_{6} \mathrm{~S}: \mathrm{C}, 70.18 ; \mathrm{H}, 7.28 ; \mathrm{N}, 8.93$. Found: C, 70.35; $\mathrm{H}, 7.03 ; \mathrm{N}, 9.22$.

4.6.9. (5R,3S)-1-Benzyl-5-((S)-1-((tert-butoxycarbonyl)amino-2-(1-(tert-butoxycarbonyl)indol-3-yl)ethyl-4-(tert-butoxycarbonyl)methyl -3-(3-(2-((2,2,4,6,7-pentamethyl-2,3dihydrobenzofuran-5-yl)sulfonyl)guanidino)propyl)-2-oxopiperazine [(R)-20a]

Amorphous solid (172 mg, 50\%); $[\alpha]_{\mathrm{D}}^{20}+3.1\left(c\right.$ 1.1, $\left.\mathrm{CH}_{2} \mathrm{Cl}_{2}\right)$; HPLC [Sunfire $\mathrm{C}_{18}(4.6 \times$ $150 \mathrm{~mm}, 3.5 \mu \mathrm{m}), 80-100 \%$ gradient of solvent $\mathrm{A}$ in $\mathrm{B}, 30 \mathrm{~min}] t_{\mathrm{R}} 12.48 \mathrm{~min} ;{ }^{1} \mathrm{H}$ NMR (400 $\left.\mathrm{MHz},\left(\mathrm{CD}_{3}\right)_{2} \mathrm{CO}\right) \delta(\mathrm{ppm}): 1.22$ (s, 9H, Boc), 1.42 [s, 6H, 2CH $\left.(\mathrm{Pbf})\right], 1.46$ (s, 9H, $\left.{ }^{\mathrm{t}} \mathrm{Bu}\right), 1.65$ [s, 3H, Boc (Ind)], 1.90 [m, 4H, $\beta-\mathrm{H}$ and $\gamma-\mathrm{H}(\mathrm{Arg})], 2.03$ [s, 3H, $\left.\mathrm{CH}_{3}(\mathrm{Pbf})\right], 2.50$ [s, 3H, $\mathrm{CH}_{3}$ (Pbf)], 2.58 [s, 3H, CH 3 (Pbf)], 2.78 (m, 1H, CH$_{2}$-Ind), 2.93 [s, 2H, $\mathrm{CH}_{2}$ (Pbf)], 3.19 (d, 1H, J $\left.=16 \mathrm{~Hz}, 4-\mathrm{CH}_{2}\right), 3.29(\mathrm{~m}, 1 \mathrm{H}, 6-\mathrm{H}), 3.32[\mathrm{~m}, 1 \mathrm{H}, 3-\mathrm{H}], 3.34[\mathrm{~m}, 2 \mathrm{H}, \delta-\mathrm{H}(\mathrm{Arg})], 3.41(\mathrm{~m}$, 1H, 6-H), 3.50 (m, 1H, 5-H), 3.56 (m, 1H, $\mathrm{CH}_{2}$-Ind), 3.64 (d, $\left.1 \mathrm{H}, J=16 \mathrm{~Hz}, 4-\mathrm{CH}_{2}\right), 3.90$ (m, $1 \mathrm{H}, 5-\mathrm{CH}), 4.37\left[\mathrm{~d}, 1 \mathrm{H}, J=14.5 \mathrm{~Hz}, 1-\mathrm{CH}_{2}(\mathrm{Bn})\right], 4.84\left[\mathrm{~d}, 1 \mathrm{H}, J=14.5 \mathrm{~Hz}, 1-\mathrm{CH}_{2}(\mathrm{Bn})\right]$, $5.97(\mathrm{~d}, 1 \mathrm{H}, J=9.5 \mathrm{~Hz}, N H \mathrm{Boc}), 6.52\left[\mathrm{~m}, 3 \mathrm{H}, \mathrm{NHC}\left(\mathrm{NH}_{2}\right)=\mathrm{N}\right], 7.20(\mathrm{t}, 1 \mathrm{H}, J=7.5 \mathrm{~Hz}, \mathrm{Ar})$, 7.25-7.38 (m, 6H, Ar), 7.49 (s, 1H, Ar), 7.58 (d, 1H, J= 7.5 Hz, Ar), 8.11 (d, 1H, J= 7.5 Hz, Ar). ${ }^{13} \mathrm{C}$ NMR (100 MHz, $\left.\left(\mathrm{CD}_{3}\right)_{2} \mathrm{CO}\right) \delta(\mathrm{ppm}): 12.6,18.2,19.5\left[3 \mathrm{CH}_{3}(\mathrm{Pbf})\right], 27.5\left[\mathrm{CH}_{2}\right.$-Ind and $\left.\mathrm{C}_{\gamma}(\mathrm{Arg})\right], 28.2\left[3 \mathrm{CH}_{3}\left({ }^{\mathrm{t}} \mathrm{Bu}\right)\right], 28.3\left[3 \mathrm{CH}_{3}(\mathrm{Boc})\right], 28.5\left[\mathrm{C}_{\beta}(\mathrm{Arg})\right.$ and $\left.2 \mathrm{CH}_{3}(\mathrm{Pbf})\right], 28.7$ [3 $\left.\mathrm{CH}_{3}(\mathrm{Boc})\right], 41.6\left[\mathrm{C}_{\delta}(\mathrm{Arg})\right], 43.6\left[\mathrm{CH}_{2}(\mathrm{Pbf})\right], 46.1\left(\mathrm{C}_{6}\right), 50.2\left[1-\mathrm{CH}_{2}(\mathrm{Bn})\right], 51.7\left(4-\mathrm{CH}_{2}\right)$, $51.9\left(\mathrm{C}_{5}-\mathrm{CH}\right), 55.8\left(\mathrm{C}_{5}\right), 65.2\left(\mathrm{C}_{3}\right), 78.8$ [C (Boc)], 81.7 [C ( $\left.\left.{ }^{\mathrm{t}} \mathrm{Bu}\right)\right], 83.9$ [C (Boc)], 86.9 [C (Pbf)], 115.8 [CH (Ar)], 117.4 [C (Pbf)], 118.9 [C (Ar)], 120.0, 123.1, 124.5, 124.9 [4CH (Ar)], 125.3 [C (Pbf)], 127.1, 128.7, 129.4 [5CH (Ar)], 131.9 [C (Ar)], 132.8, 135.6 [2C (Pbf)], 136.3 [C (Ar)], 138.6 [C (Pbf)], 138.7 [C (Ar)], 150.3, 156.5 [2CO (2Boc)], 157.4 [C $\left.\left(\mathrm{NHC}\left(\mathrm{NH}_{2}\right)=\mathrm{N}\right)\right], 158.9$ [C (Pbf)], $170.6\left(\mathrm{C}_{2}\right), 170.9\left(\mathrm{CO}_{2}\right)$; ES-MS m/z $1015.2[\mathrm{M}+1]^{+}$; Anal. calcd. for $\mathrm{C}_{54} \mathrm{H}_{75} \mathrm{~N}_{7} \mathrm{O}_{10} \mathrm{~S}$ : C, 63.94; H, 7.45; N, 9.67. Found: C, 64.22; H, 7.63; N, 9.36.

4.6.10. (5S,3S)-1-Benzyl-5-((S)-1-((tert-butoxycarbonyl)amino-2-(1-(tert-butoxycarbonyl)indol-3-yl)ethyl-4-(tert-butoxycarbonyl)methyl -3-(3-(2-((2,2,4,6,7-pentamethyl-2,3dihydrobenzofuran-5-yl)sulfonyl)guanidino)propyl)-2-oxopiperazine [(S)-20a] 
Amorphous solid (197 mg, 57\%); $[\alpha]_{\mathrm{D}}{ }^{20}+20\left(c\right.$ 1.1, $\left.\mathrm{CH}_{2} \mathrm{Cl}_{2}\right)$; HPLC [Sunfire $\mathrm{C}_{18}(4.6 \times$ $150 \mathrm{~mm}, 3.5 \mu \mathrm{m}), 80-100 \%$ gradient of solvent $\mathrm{A}$ in $\mathrm{B}, 30 \mathrm{~min}] t_{\mathrm{R}} 13.67 \mathrm{~min} ;{ }^{1} \mathrm{H}$ NMR (400 $\left.\mathrm{MHz},\left(\mathrm{CD}_{3}\right)_{2} \mathrm{CO}\right) \delta(\mathrm{ppm}): 1.27$ (s, 9H, Boc), 1.43 [s, 6H, 2CH$\left.(\mathrm{Pbf})\right], 1.45$ (s, 9H, $\left.{ }^{\mathrm{t}} \mathrm{Bu}\right), 1.65$ [s, 3H, Boc (Ind)], 1.76 [m, 2H, $\gamma-\mathrm{H}(\mathrm{Arg})], 1.80[\mathrm{~m}, 2 \mathrm{H}, \beta-\mathrm{H}(\mathrm{Arg})], 2.04$ [s, 3H, $\left.\mathrm{CH}_{3}(\mathrm{Pbf})\right]$, 2.49 [s, 3H, $\left.\mathrm{CH}_{3}(\mathrm{Pbf})\right], 2.58$ [s, 3H, $\left.\mathrm{CH}_{3}(\mathrm{Pbf})\right], 2.68$ (dd, $1 \mathrm{H}, J=11$ and $15 \mathrm{~Hz}, \mathrm{CH}_{2}$-Ind), 2.95 [s, 2H, $\mathrm{CH}_{2}$ (Pbf)], 3.13 (m, 1H, $\mathrm{CH}_{2}$-Ind), 3.26 [m, 2H, $\delta$-H (Arg)], 3.28 (m, 1H, 5-H), $3.49\left(\mathrm{~m}, 3 \mathrm{H}, 3-\mathrm{H}\right.$ and 6-H), $3.58\left(\mathrm{~d}, 1 \mathrm{H}, J=18 \mathrm{~Hz}, 4-\mathrm{CH}_{2}\right), 3.68\left(\mathrm{~d}, 1 \mathrm{H}, J=18 \mathrm{~Hz}, 4-\mathrm{CH}_{2}\right)$, $4.01(\mathrm{~m}, 1 \mathrm{H}, 5-\mathrm{CH}), 4.59\left[\mathrm{~d}, 1 \mathrm{H}, J=14.5 \mathrm{~Hz}, 1-\mathrm{CH}_{2}(\mathrm{Bn})\right], 4.69\left[\mathrm{~d}, 1 \mathrm{H}, J=14.5 \mathrm{~Hz}, 1-\mathrm{CH}_{2}\right.$ $(\mathrm{Bn})], 6.15(\mathrm{~d}, 1 \mathrm{H}, J=8 \mathrm{~Hz}, N H \mathrm{Boc}), 6.49$ [m, 3H, NHC( $\left.\left.\mathrm{NH}_{2}\right)=\mathrm{N}\right], 7.18-7.38(\mathrm{~m}, 7 \mathrm{H}, \mathrm{Ar})$, 7.47 (s, 1H, Ar), 7.58 (d, 1H, $J=7.5 \mathrm{~Hz}, \mathrm{Ar}), 8.11$ (d, 1H, $J=7.5 \mathrm{~Hz}, \mathrm{Ar}) .{ }^{13} \mathrm{C}$ NMR (100 $\left.\mathrm{MHz},\left(\mathrm{CD}_{3}\right)_{2} \mathrm{CO}\right) \delta(\mathrm{ppm}): 12.6,18.2,19.5$ [3 $\left.\mathrm{CH}_{3}(\mathrm{Pbf})\right], 25.3\left(\mathrm{CH}_{2}\right.$-Ind), 26.5 [C $\left.\mathrm{C}_{\gamma}(\mathrm{Arg})\right]$, $28.3\left[3 \mathrm{CH}_{3}\left({ }^{\mathrm{t}} \mathrm{Bu}\right)\right.$ and $\left.3 \mathrm{CH}_{3}(\mathrm{Boc})\right], 28.6\left[2 \mathrm{CH}_{3}(\mathrm{Pbf})\right], 28.7$ [3 $\left.\mathrm{CH}_{3}(\mathrm{Boc})\right], 32.2\left[\mathrm{C}_{\beta}(\mathrm{Arg})\right]$, $41.7\left[\mathrm{C}_{\delta}(\mathrm{Arg})\right], 43.6\left[\mathrm{CH}_{2}(\mathrm{Pbf})\right], 44.9\left(\mathrm{C}_{6}\right), 50.0\left[1-\mathrm{CH}_{2}(\mathrm{Bn})\right], 52.0\left(\mathrm{C}_{5}-\mathrm{CH}\right), 56.6\left(4-\mathrm{CH}_{2}\right)$, $60.9\left(\mathrm{C}_{5}\right), 65.0\left(\mathrm{C}_{3}\right), 78.9$ [C (Boc)], 81.7 [C ( $\left.\left.{ }^{\mathrm{t}} \mathrm{Bu}\right)\right], 84.0$ [C (Boc)], 86.9 [C (Pbf)], 115.9 [CH (Ar)], 117.4 [C (Pbf)], 119.0 [C (Ar)], 119.9, 123.3, 124.1, 125.0 [4CH (Ar)], 125.3 [C (Pbf)], 128.1, 128.8, 129.4 [5CH (Ar)], 131.7 [C (Ar)], 132.8, 135.7 [2C (Pbf)], 136.3, 138.5 [2C (Ar)], 138.7 [C (Pbf)], 150.3, 156.4 [2CO (2Boc)], 157.3 [C $\left.\left(\mathrm{NHC}\left(\mathrm{NH}_{2}\right)=\mathrm{N}\right)\right], 158.9[\mathrm{C}$ (Pbf)], $171.0\left(\mathrm{C}_{2}\right), 171.3\left(\mathrm{CO}_{2}\right)$; ES-MS m/z $1015.2[\mathrm{M}+1]^{+}$; Anal. calcd. for $\mathrm{C}_{54} \mathrm{H}_{75} \mathrm{~N}_{7} \mathrm{O}_{10} \mathrm{~S}$ : C, 63.94; H, 7.45; N, 9.67. Found: C, 63.76; H, 7.69; N, 9.92.

\subsubsection{1. (5R,3S)-1-Benzyl-5-((S)-1-((tert-butoxycarbonyl)amino-2-(1-(tert-butoxycarbonyl)-} indol-3-yl)ethyl-4-(tert-butoxycarbonyl)methyl-3-(3-(3-benzyl-2-((2,2,4,6,7-pentamethyl2,3-dihydrobenzofuran-5-yl)sulfonyl)guanidino)propyl)-2-oxopiperazine [(R)-22a]

Amorphous solid (5:1 isomeric mixture, $60 \mathrm{mg}, 16 \%)$; HPLC [Sunfire $\mathrm{C}_{18}(4.6 \times 150 \mathrm{~mm}$, $3.5 \mu \mathrm{m}$ ), 80-100\% gradient of solvent $\mathrm{A}$ in $\mathrm{B}, 30 \mathrm{~min}] t_{\mathrm{R}} 20.05$ min (major isomer) and 19.34 min (minor isomer); ${ }^{1} \mathrm{H}$ NMR $\left(400 \mathrm{MHz},\left(\mathrm{CD}_{3}\right)_{2} \mathrm{CO}\right) \delta(\mathrm{ppm})$ Major isomer: $1.20(\mathrm{~s}, 9 \mathrm{H}$, Boc), 1.42 [s, 6H, 2CH3 (Pbf)], 1.46 (s, 9H, ${ }^{\mathrm{t}} \mathrm{Bu}$ ), 1.64 [s, 3H, Boc (Ind)], 1.90 [m, 4H, $\beta-\mathrm{H}$ and $\gamma-\mathrm{H}(\mathrm{Arg})], 2.00\left[\mathrm{~s}, 3 \mathrm{H}, \mathrm{CH}_{3}(\mathrm{Pbf})\right], 2.44$ [s, 3H, $\left.\mathrm{CH}_{3}(\mathrm{Pbf})\right], 2.50$ [s, 3H, $\left.\mathrm{CH}_{3}(\mathrm{Pbf})\right], 2.79$ (m, 1H, $\mathrm{CH}_{2}$-Ind), 2.93 [s, 2H, $\mathrm{CH}_{2}$ (Pbf)], 3.20 (d, 1H, J = $\left.16 \mathrm{~Hz}, 4-\mathrm{CH}_{2}\right), 3.30$ (m, 1H, 6-H), $3.34[\mathrm{~m}, 1 \mathrm{H}, 3-\mathrm{H}], 3.38[\mathrm{~m}, 1 \mathrm{H}, \delta-\mathrm{H}(\mathrm{Arg})], 3.41(\mathrm{~m}, 1 \mathrm{H}, 6-\mathrm{H}), 3.47$ [m, 1H, $\delta-\mathrm{H}(\mathrm{Arg})], 3.49$ (m, 1H, 5-H), 3.57 (m, 1H, $\mathrm{CH}_{2}$-Ind), 3.65 (d, 1H, J = $\left.16 \mathrm{~Hz}, 4-\mathrm{CH}_{2}\right), 3.90$ (m, 1H, 5-CH), $4.38\left[\mathrm{dd}, 1 \mathrm{H}, J=6\right.$ and $\left.15 \mathrm{~Hz}, \mathrm{CH}_{2}(\mathrm{NHC}(\mathrm{NHBn})=\mathrm{N})\right], 4.40\left[\mathrm{~d}, 1 \mathrm{H}, J=14.5 \mathrm{~Hz}, 1-\mathrm{CH}_{2}\right.$ $(\mathrm{Bn})], 4.46\left[\mathrm{dd}, 1 \mathrm{H}, J=6\right.$ and $\left.15 \mathrm{~Hz}, \mathrm{CH}_{2}(\mathrm{NHC}(\mathrm{NHBn})=\mathrm{N})\right], 4.83[\mathrm{~d}, 1 \mathrm{H}, J=14.5 \mathrm{~Hz}, 1-$ 
$\left.\mathrm{CH}_{2}(\mathrm{Bn})\right], 5.96(\mathrm{~d}, 1 \mathrm{H}, J=9.5 \mathrm{~Hz}, N H \mathrm{Boc}), 7.09-7.40[\mathrm{~m}, 14 \mathrm{H}, \mathrm{Ar}$ and $N H \mathrm{C}(N H \mathrm{Bn})=\mathrm{N}]$, 7.50 (s, 1H, Ar), 7.61 (d, 1H, $J=7.5 \mathrm{~Hz}, \mathrm{Ar}), 8.11$ (d, 1H, $J=7.5 \mathrm{~Hz}, \mathrm{Ar})$. Minor isomer: 1.23 (s, 9H, Boc), 1.43 [s, 6H, 2CH $\mathrm{CH}_{3}$ (Pb)], 1.44 (s, 9H, $\left.{ }^{\mathrm{B}} \mathrm{Bu}\right), 1.66$ [s, 3H, Boc (Ind)], 1.90 [m, 4H, $\beta-\mathrm{H}$ and $\gamma-\mathrm{H}(\mathrm{Arg})], 2.00\left[\mathrm{~s}, 3 \mathrm{H}, \mathrm{CH}_{3}(\mathrm{Pbf})\right], 2.45$ [s, 3H, $\left.\mathrm{CH}_{3}(\mathrm{Pbf})\right], 2.50$ [s, 3H, $\mathrm{CH}_{3}$ (Pbf)], 2.79 (m, 1H, $\mathrm{CH}_{2}$-Ind), 2.95 [s, 2H, $\mathrm{CH}_{2}$ (Pbf)], 3.22 (d, 1H, J = $16 \mathrm{~Hz}, 4-\mathrm{CH}_{2}$ ), $3.30(\mathrm{~m}, 1 \mathrm{H}, 6-\mathrm{H}), 3.34[\mathrm{~m}, 1 \mathrm{H}, 3-\mathrm{H}], 3.38[\mathrm{~m}, 1 \mathrm{H}, \delta-\mathrm{H}(\mathrm{Arg})], 3.41(\mathrm{~m}, 1 \mathrm{H}, 6-\mathrm{H}), 3.47$ [m, $1 \mathrm{H}, \delta-\mathrm{H}(\mathrm{Arg})], 3.49(\mathrm{~m}, 1 \mathrm{H}, 5-\mathrm{H}), 3.57\left(\mathrm{~m}, 1 \mathrm{H}, \mathrm{CH}_{2}-\mathrm{Ind}\right), 3.65\left(\mathrm{~d}, 1 \mathrm{H}, J=16 \mathrm{~Hz}, 4-\mathrm{CH}_{2}\right)$, $3.90(\mathrm{~m}, 1 \mathrm{H}, 5-\mathrm{CH}), 4.35\left[\mathrm{~d}, 1 \mathrm{H}, J=14.5 \mathrm{~Hz}, 1-\mathrm{CH}_{2}(\mathrm{Bn})\right], 4.68\left[\mathrm{~d}, 1 \mathrm{H}, J=16 \mathrm{~Hz}, \mathrm{CH}_{2}\right.$ $(\mathrm{NHC}(\mathrm{NHBn})=\mathrm{N})], 4.77\left[\mathrm{~d}, 1 \mathrm{H}, J=16 \mathrm{~Hz}, \mathrm{CH}_{2}(\mathrm{NHC}(\mathrm{NHBn})=\mathrm{N})\right], 4.85[\mathrm{~d}, 1 \mathrm{H}, J=14.5 \mathrm{~Hz}$, $\left.1-\mathrm{CH}_{2}(\mathrm{Bn})\right], 5.94(\mathrm{~d}, 1 \mathrm{H}, J=9.5 \mathrm{~Hz}, N H \mathrm{Boc}), 7.09-7.40[\mathrm{~m}, 14 \mathrm{H}, \mathrm{Ar}$ and $N H \mathrm{C}(N H \mathrm{Bn})=\mathrm{N}]$, 7.47 (s, 1H, Ar), 7.55 (d, 1H, $J=7.5 \mathrm{~Hz}, \mathrm{Ar}), 8.11$ (d, 1H, $J=7.5 \mathrm{~Hz}, \mathrm{Ar}) ;{ }^{13} \mathrm{C}$ NMR (100 $\left.\mathrm{MHz},\left(\mathrm{CD}_{3}\right)_{2} \mathrm{CO}\right) \delta$ (ppm) Major isomer: 12.5, 18.3, 19.6 [3 $\left.\mathrm{CH}_{3}(\mathrm{Pbf})\right], 27.2\left(\mathrm{CH}_{2}\right.$-Ind), 27.6 $\left[\mathrm{C}_{\gamma}(\mathrm{Arg})\right], 28.3\left[3 \mathrm{CH}_{3}\left({ }^{\mathrm{t}} \mathrm{Bu}\right)\right], 28.7\left[3 \mathrm{CH}_{3}(\mathrm{Boc})\right], 28.4\left[\mathrm{C}_{\beta}(\mathrm{Arg})\right.$ and $\left.2 \mathrm{CH}_{3}(\mathrm{Pbf})\right], 28.7\left[3 \mathrm{CH}_{3}\right.$ (Boc) $], 41.8\left[\mathrm{C}_{\delta}(\mathrm{Arg})\right], 43.6\left[\mathrm{CH}_{2}(\mathrm{Pbf})\right], 45.4\left[\mathrm{CH}_{2}(\mathrm{NHC}(\mathrm{NHBn})=\mathrm{N})\right], 46.2\left(\mathrm{C}_{6}\right), 50.3[1-$ $\mathrm{CH}_{2}(\mathrm{Bn})$ ], $51.6\left(4-\mathrm{CH}_{2}\right), 52.0\left(\mathrm{C}_{5}-\mathrm{CH}\right), 55.5\left(\mathrm{C}_{5}\right), 65.0\left(\mathrm{C}_{3}\right), 78.8$ [C (Boc)], $81.7\left[\mathrm{C}\left({ }^{\mathrm{t}} \mathrm{Bu}\right)\right]$, 83.9 [C (Boc)], 86.9 [C (Pbf)], 115.8 [CH (Ar)], 117.5 [C (Pbf)], 118.6 [C (Ar)], 120.0, 123.1, 124.7, 124.9 [4CH (Ar)], 125.3 [C (Pbf)], 128.1, 128.7, 129.5 [10CH (Ar)], 131.9 [C (Ar)], 132.8, 135.6 [2C (Pbf)], 136.4 [C (Ar)], 138.6 [C (Pbf)], 138.8 [2C (Ar)], 150.3, 156.1 [2CO $(2 \mathrm{Boc})], 156.5[\mathrm{C}(\mathrm{NHC}(\mathrm{NHBn})=\mathrm{N})], 158.9$ [C $(\mathrm{Pbf})], 170.5\left(\mathrm{C}_{2}\right), 171.0\left(\mathrm{CO}_{2}\right)$. Minor isomer: 12.5, 18.3, $19.6\left[3 \mathrm{CH}_{3}(\mathrm{Pbf})\right], 27.2\left(\mathrm{CH}_{2}\right.$-Ind), $27.6\left[\mathrm{C}_{\gamma}(\mathrm{Arg})\right], 28.3\left[3 \mathrm{CH}_{3}\left({ }^{\mathrm{t}} \mathrm{Bu}\right)\right]$, $28.7\left[3 \mathrm{CH}_{3}(\mathrm{Boc})\right], 28.4\left[\mathrm{C}_{\beta}(\mathrm{Arg})\right.$ and $\left.2 \mathrm{CH}_{3}(\mathrm{Pbf})\right], 28.7$ [3 $\left.\mathrm{CH}_{3}(\mathrm{Boc})\right], 41.8\left[\mathrm{C}_{\delta}(\mathrm{Arg})\right], 43.6$ $\left[\mathrm{CH}_{2}(\mathrm{Pbf})\right], 46.2\left(\mathrm{C}_{6}\right), 50.3\left[1-\mathrm{CH}_{2}(\mathrm{Bn})\right], 51.6\left[4-\mathrm{CH}_{2}\right.$ and $\left.\mathrm{CH}_{2}(\mathrm{NHC}(\mathrm{NHBn})=\mathrm{N})\right], 52.0\left(\mathrm{C}_{5^{-}}\right.$ $\mathrm{CH}), 55.5\left(\mathrm{C}_{5}\right), 65.0\left(\mathrm{C}_{3}\right), 78.8$ [C (Boc)], 81.7 [C ( $\left.\left.{ }^{\mathrm{t}} \mathrm{Bu}\right)\right], 83.9$ [C (Boc)], 86.9 [C (Pbf)], 115.8 [CH (Ar)], 117.5 [C (Pbf)], 118.8 [C (Ar)], 120.0, 123.1, 124.5, 124.9 [4CH (Ar)], 125.3 [C (Pbf)], 128.1, 128.8, 129.2 [10CH (Ar)], 131.9 [C (Ar)], 132.7, 135.4 [2C (Pbf)], 136.4 [C $(\mathrm{Ar})], \quad 138.5 \quad[\mathrm{C} \quad(\mathrm{Pbf})], \quad 138.8 \quad[2 \mathrm{C} \quad(\mathrm{Ar})], \quad 150.3, \quad 156.1 \quad[2 \mathrm{CO} \quad$ (2Boc) $], \begin{array}{lllll}156.5 & {[\mathrm{C}}\end{array}$ $(\mathrm{NHC}(\mathrm{NHBn})=\mathrm{N})], 158.9$ [C $(\mathrm{Pbf})], 170.4\left(\mathrm{C}_{2}\right), 171.0\left(\mathrm{CO}_{2}\right)$; ES-MS m/z $1105.3[\mathrm{M}+1]^{+}$; Anal. calcd. for $\mathrm{C}_{61} \mathrm{H}_{81} \mathrm{~N}_{7} \mathrm{O}_{10} \mathrm{~S}$ : C, 66.34; H, 7.39; N, 8.88. Found: C, 66.58; H, 7.67; N, 8.61.

4.6.12. (5S,3S)-1-Benzyl-5-((S)-1-((tert-butoxycarbonyl)amino-2-(1-(tert-butoxycarbonyl)-

indol-3-yl)ethyl-4-(tert-butoxycarbonyl)methyl-3-(3-(3-benzyl-2-((2,2,4,6,7-pentamethyl2,3-dihydrobenzofuran-5-yl)sulfonyl)guanidino)propyl)-2-oxopiperazine [(S)-22a] 
Amorphous solid (3:1 isomeric mixture, $75 \mathrm{mg}, 20 \%)$; HPLC [Sunfire $\mathrm{C}_{18}(4.6 \times 150 \mathrm{~mm}$, $3.5 \mu \mathrm{m}$ ), $80-100 \%$ gradient of solvent $\mathrm{A}$ in $\mathrm{B}, 30 \mathrm{~min}] t_{\mathrm{R}} 21.04$ min (major isomer) and 21.82 min (minor isomer); ${ }^{1} \mathrm{H}$ NMR $\left(400 \mathrm{MHz},\left(\mathrm{CD}_{3}\right)_{2} \mathrm{CO}\right) \delta(\mathrm{ppm})$ Major isomer: 1.27 (s, 9H, Boc), 1.43 [s, 6H, 2CH3 (Pbf)], 1.46 (s, 9H, $\left.{ }^{\mathrm{t}} \mathrm{Bu}\right), 1.65$ [s, 3H, Boc (Ind)], 1.79 [m, 2H, $\gamma-\mathrm{H}$ (Arg)], $1.81[\mathrm{~m}, 2 \mathrm{H}, \beta-\mathrm{H}(\mathrm{Arg})], 2.01\left[\mathrm{~s}, 3 \mathrm{H}, \mathrm{CH}_{3}(\mathrm{Pbf})\right], 2.43$ [s, 3H, $\left.\mathrm{CH}_{3}(\mathrm{Pbf})\right], 2.51$ [s, 3H, $\mathrm{CH}_{3}$ (Pbf)], 2.70 (m, 1H, $\mathrm{CH}_{2}$-Ind), 2.91 [s, 2H, $\mathrm{CH}_{2}$ (Pbf)], 3.15 (m, 1H, $\mathrm{CH}_{2}$-Ind), 3.28 (m, $1 \mathrm{H}, 5-\mathrm{H}), 3.31[\mathrm{~m}, 2 \mathrm{H}, \delta-\mathrm{H}(\mathrm{Arg})], 3.48(\mathrm{~m}, 2 \mathrm{H}, 6-\mathrm{H}), 3.54[\mathrm{~m}, 1 \mathrm{H}, 3-\mathrm{H}], 3.60$ (m, 1H, 4$\left.\mathrm{CH}_{2}\right), 3.70\left(\mathrm{~m}, 1 \mathrm{H}, 4-\mathrm{CH}_{2}\right), 4.02(\mathrm{~m}, 1 \mathrm{H}, 5-\mathrm{CH}), 4.42\left[\mathrm{~d}, 2 \mathrm{H}, J=5.5 \mathrm{~Hz}, \mathrm{CH}_{2}\right.$ $(\mathrm{NHC}(\mathrm{NHBn})=\mathrm{N})], 4.58\left[\mathrm{~m}, 1 \mathrm{H}, 1-\mathrm{CH}_{2}(\mathrm{Bn})\right], 4.70\left[\mathrm{~m}, 1 \mathrm{H}, 1-\mathrm{CH}_{2}(\mathrm{Bn})\right], 6.14(\mathrm{~d}, 1 \mathrm{H}, J=8$ $\mathrm{Hz}, N H \mathrm{Boc}), 7.10-7.41[\mathrm{~m}, 14 \mathrm{H}, \mathrm{Ar}$ and $N H \mathrm{C}(N H \mathrm{Bn})=\mathrm{N}], 7.50(\mathrm{~s}, 1 \mathrm{H}, \mathrm{Ar}), 7.59(\mathrm{~d}, 1 \mathrm{H}, J=$ $7.5 \mathrm{~Hz}, \mathrm{Ar}), 8.11(\mathrm{~d}, 1 \mathrm{H}, J=7.5 \mathrm{~Hz}, \mathrm{Ar}$ ). Minor isomer: 1.27 (s, 9H, Boc), 1.44 [s, 6H, 2CH (Pbf)], 1.45 (s, 9H, $\left.{ }^{\mathrm{B}} \mathrm{Bu}\right), 1.65$ [s, 3H, Boc (Ind)], 1.79 [m, 2H, $\gamma-\mathrm{H}$ (Arg)], 1.81 [m, 2H, $\beta-\mathrm{H}$ (Arg)], 2.01 [s, 3H, $\left.\mathrm{CH}_{3}(\mathrm{Pbf})\right], 2.43$ [s, 3H, $\mathrm{CH}_{3}$ (Pbf)], 2.51 [s, 3H, $\left.\mathrm{CH}_{3}(\mathrm{Pbf})\right], 2.70$ (m, 1H, $\mathrm{CH}_{2}$-Ind), 2.96 [s, 2H, CH 2 (Pbf)], 3.15 (m, 1H, $\mathrm{CH}_{2}$-Ind), 3.28 (m, 1H, 5-H), 3.31 [m, 2H, $\delta$ $\mathrm{H}(\mathrm{Arg})], 3.48(\mathrm{~m}, 2 \mathrm{H}, 6-\mathrm{H}), 3.54[\mathrm{~m}, 1 \mathrm{H}, 3-\mathrm{H}], 3.60\left(\mathrm{~m}, 1 \mathrm{H}, 4-\mathrm{CH}_{2}\right), 3.70\left(\mathrm{~m}, 1 \mathrm{H}, 4-\mathrm{CH}_{2}\right)$, $4.02(\mathrm{~m}, 1 \mathrm{H}, 5-\mathrm{CH}), 4.52\left[\mathrm{~m}, 1 \mathrm{H}, 1-\mathrm{CH}_{2}(\mathrm{Bn})\right], 4.60\left[\mathrm{~m}, 1 \mathrm{H}, \mathrm{CH}_{2}(\mathrm{NHC}(\mathrm{NHBn})=\mathrm{N})\right], 4.66$ $\left[\mathrm{m}, 1 \mathrm{H}, \mathrm{CH}_{2}(\mathrm{NHC}(\mathrm{NHBn})=\mathrm{N})\right], 4.70\left[\mathrm{~m}, 1 \mathrm{H}, 1-\mathrm{CH}_{2}(\mathrm{Bn})\right], 6.14(\mathrm{~d}, 1 \mathrm{H}, J=8 \mathrm{~Hz}, \mathrm{NHBoc})$, 7.10-7.41 [m, 14H, Ar and $N H C(N H B n)=\mathrm{N}], 7.50(\mathrm{~s}, 1 \mathrm{H}, \mathrm{Ar}), 7.59(\mathrm{~d}, 1 \mathrm{H}, J=7.5 \mathrm{~Hz}, \mathrm{Ar})$, $8.11(\mathrm{~d}, 1 \mathrm{H}, J=7.5 \mathrm{~Hz}, \mathrm{Ar}) ;{ }^{13} \mathrm{C} \mathrm{NMR}\left(100 \mathrm{MHz},\left(\mathrm{CD}_{3}\right)_{2} \mathrm{CO}\right) \delta$ (ppm) Major isomer: 11.8 , 17.5, $18.7\left[3 \mathrm{CH}_{3}(\mathrm{Pbf})\right], 24.7\left(\mathrm{CH}_{2}\right.$-Ind), $25.8\left[\mathrm{C}_{\gamma}(\mathrm{Arg})\right], 27.6\left[3 \mathrm{CH}_{3}\left({ }^{\mathrm{t}} \mathrm{Bu}\right)\right.$ and $\left.3 \mathrm{CH}_{3}(\mathrm{Boc})\right]$, $27.9\left[2 \mathrm{CH}_{3}(\mathrm{Pbf})\right], 28.0\left[3 \mathrm{CH}_{3}(\mathrm{Boc})\right], 31.3\left[\mathrm{C}_{\beta}(\mathrm{Arg})\right], 41.4\left[\mathrm{C}_{\delta}(\mathrm{Arg})\right], 43.0\left[\mathrm{CH}_{2}(\mathrm{Pbf})\right], 44.4$ $\left(\mathrm{C}_{6}\right), 44.9\left[\mathrm{CH}_{2}(\mathrm{NHC}(\mathrm{NHBn})=\mathrm{N})\right], 49.6\left[1-\mathrm{CH}_{2}(\mathrm{Bn})\right], 51.5\left(\mathrm{C}_{5}-\mathrm{CH}\right), 56.1\left(4-\mathrm{CH}_{2}\right), 60.4$ $\left(\mathrm{C}_{5}\right), 64.1\left(\mathrm{C}_{3}\right), 78.3$ [C (Boc)], $81.2\left[\mathrm{C}\left({ }^{\mathrm{t}} \mathrm{Bu}\right)\right], 83.4$ [C (Boc)], 86.2 [C (Pbf)], $115.2[\mathrm{CH}$ (Ar)], 116.8 [C (Pbf)], 118.3 [C (Ar)], 119.3, 122.6, 123.6, 124.3 [4CH (Ar)], 124.6 [C (Pbf)], 127.5, 128.1, 128.4, 128.7 [10CH (Ar)], 131.1 [C (Ar)], 132.2, 135.2 [2C (Pbf)], 135.8 [C $(\mathrm{Ar})], \quad 137.8[2 \mathrm{C} \quad(\mathrm{Ar})], \quad 138.0 \quad[\mathrm{C} \quad(\mathrm{Pbf})], \quad 149.6, \quad 155.3 \quad[2 \mathrm{CO} \quad(2 \mathrm{Boc})], \quad 155.8 \quad[\mathrm{C}$ $(\mathrm{NHC}(\mathrm{NHBn})=\mathrm{N})], 158.3[\mathrm{C}(\mathrm{Pbf})], 170.4\left(\mathrm{C}_{2}\right), 170.6\left(\mathrm{CO}_{2}\right)$. Minor isomer: 11.8, 17.5, 18.7 [3 $\left.3 \mathrm{CH}_{3}(\mathrm{Pbf})\right], 24.7\left(\mathrm{CH}_{2}-\mathrm{Ind}\right), 25.8\left[\mathrm{C}_{\gamma}(\mathrm{Arg})\right], 27.6\left[3 \mathrm{CH}_{3}\left({ }^{\mathrm{t}} \mathrm{Bu}\right)\right.$ and $\left.3 \mathrm{CH}_{3}(\mathrm{Boc})\right], 27.9$ [2 $\mathrm{CH}_{3}$ (Pbf)], 28.0 [3 $\mathrm{CH}_{3}$ (Boc)], $31.3\left[\mathrm{C}_{\beta}(\mathrm{Arg})\right], 41.4\left[\mathrm{C}_{\delta}(\mathrm{Arg})\right], 43.0\left[\mathrm{CH}_{2}(\mathrm{Pbf})\right], 44.4\left(\mathrm{C}_{6}\right), 49.6$ $\left[1-\mathrm{CH}_{2}(\mathrm{Bn})\right.$ and $\left.\mathrm{CH}_{2}(\mathrm{NHC}(\mathrm{NHBn})=\mathrm{N})\right], 51.5\left(\mathrm{C}_{5}-\mathrm{CH}\right), 56.1\left(4-\mathrm{CH}_{2}\right), 60.4\left(\mathrm{C}_{5}\right), 64.1\left(\mathrm{C}_{3}\right)$, $78.3[\mathrm{C}(\mathrm{Boc})], 81.2\left[\mathrm{C}\left({ }^{\mathrm{t}} \mathrm{Bu}\right)\right], 83.4$ [C (Boc)], 86.2 [C (Pbf)], 115.2 [CH (Ar)], 116.8 [C (Pbf)], 118.3 [C (Ar)], 119.3, 122.6, 123.6, 124.3 [4CH (Ar)], 124.6 [C (Pbf)], 127.5, 128.1, 128.4, 128.7 [10CH (Ar)], 131.1 [C (Ar)], 132.2, 135.2 [2C (Pbf)], 135.8 [C (Ar)], 137.8 [2C 
(Ar)], 138.0 [C (Pbf)], 149.6, 155.3 [2CO (2Boc)], 155.8 [C $(\mathrm{NHC}(\mathrm{NHBn})=\mathrm{N})], 158.3$ [C (Pbf)], $170.4\left(\mathrm{C}_{2}\right), 170.6\left(\mathrm{CO}_{2}\right)$; ES-MS $m / z 1105.3[\mathrm{M}+1]^{+}$; Anal. calcd. for $\mathrm{C}_{61} \mathrm{H}_{81} \mathrm{~N}_{7} \mathrm{O}_{10} \mathrm{~S}$ : C, 66.34; H, 7.39; N, 8.88. Found: C, 66.07; H, 7.27; N, 9.14.

4.6.13. (5R,3S)-1-Benzyl-5-((S)-1-(tert-butoxycarbonyl)amino-2-phenylethyl)- 4-(tertbutoxycarbonyl)methyl -3-(3-(2-((2,2,4,6,7-pentamethyl-2,3-dihydrobenzofuran-5yl)sulfonyl)guanidino) propyl)-2-oxopiperazine [(R)-23a]

Amorphous solid (152 mg, 51\%); $[\alpha]_{\mathrm{D}}^{20}-2.8$ (c 1.0, $\left.\mathrm{CH}_{2} \mathrm{Cl}_{2}\right)$; HPLC [Sunfire $\mathrm{C}_{18}(4.6 \times$ $150 \mathrm{~mm}, 3.5 \mu \mathrm{m}), 80-100 \%$ gradient of solvent $\mathrm{A}$ in $\mathrm{B}, 30 \mathrm{~min}] t_{\mathrm{R}} 6.90 \mathrm{~min} ;{ }^{1} \mathrm{H}$ NMR (400 $\left.\mathrm{MHz},\left(\mathrm{CD}_{3}\right)_{2} \mathrm{CO}\right) \delta(\mathrm{ppm}): 1.21$ (s, 9H, Boc), 1.44 [s, 6H, 2CH $\left.3(\mathrm{Pbf})\right], 1.46$ (s, 9H, $\left.{ }^{\mathrm{t}} \mathrm{Bu}\right), 1.88$ $[\mathrm{m}, 4 \mathrm{H}, \beta-\mathrm{H}(\mathrm{Arg})$ and $\gamma-\mathrm{H}(\mathrm{Arg})], 2.04\left[\mathrm{~s}, 3 \mathrm{H}, \mathrm{CH}_{3}(\mathrm{Pbf})\right], 2.50\left[\mathrm{~s}, 3 \mathrm{H}, \mathrm{CH}_{3}(\mathrm{Pbf})\right], 2.58$ [s, $3 \mathrm{H}, \mathrm{CH}_{3}$ (Pbf)], 2.62 (dd, 1H, J = 11 and $14 \mathrm{~Hz}, \mathrm{CH}_{2}-\mathrm{Ph}$ ), 2.97 [s, 2H, $\left.\mathrm{CH}_{2}(\mathrm{Pbf})\right], 3.13$ (d, $\left.1 \mathrm{H}, J=16 \mathrm{~Hz}, 4-\mathrm{CH}_{2}\right), 3.22(\mathrm{~m}, 1 \mathrm{H}, 6-\mathrm{H}), 3.30$ [m, 2H, $\left.\delta-\mathrm{H}(\mathrm{Arg})\right], 3.35$ [m, 1H, 3-H], 3.37 (m, 1H, 6-H), 3.39 (m, 1H, 5-H), 3.46 (dd, $1 \mathrm{H}, J=3$ and $\left.14 \mathrm{~Hz}, \mathrm{CH}_{2}-\mathrm{Ph}\right), 3.60$ (d, $1 \mathrm{H}, J=16$ $\mathrm{Hz}, 4-\mathrm{CH}_{2}$ ), 3.82 (dt, $1 \mathrm{H}, J=3.5$ and $\left.12 \mathrm{~Hz}, 5-\mathrm{CH}\right), 4.35$ [d, $\left.1 \mathrm{H}, J=14.5 \mathrm{~Hz}, 1-\mathrm{CH}_{2}(\mathrm{Bn})\right]$, $4.83\left[\mathrm{~d}, 1 \mathrm{H}, J=14.5 \mathrm{~Hz}, 1-\mathrm{CH}_{2}(\mathrm{Bn})\right], 5.89$ (d, 1H, $\left.J=9.5 \mathrm{~Hz}, N H \mathrm{Boc}\right), 6.55[\mathrm{~m}, 3 \mathrm{H}$, $\mathrm{NHC}\left(\mathrm{NH}_{2}\right)=\mathrm{N}$ ], 7.10-7.38 (m, 10H, Ar); ${ }^{13} \mathrm{C} \mathrm{NMR}\left(100 \mathrm{MHz},\left(\mathrm{CD}_{3}\right)_{2} \mathrm{CO}\right) \delta(\mathrm{ppm}): 12.7$, 18.2, $19.5\left[3 \mathrm{CH}_{3}(\mathrm{Pbf})\right], 27.4\left[\mathrm{C}_{\gamma}(\mathrm{Arg})\right], 28.3\left[3 \mathrm{CH}_{3}\left({ }^{\mathrm{t}} \mathrm{Bu}\right)\right], 28.5\left[2 \mathrm{CH}_{3}(\mathrm{Pbf})\right], 28.7\left[3 \mathrm{CH}_{3}\right.$ (Boc)], $28.9\left[\mathrm{C}_{\beta}(\mathrm{Arg})\right], 38.0\left(\mathrm{CH}_{2}-\mathrm{Ph}\right), 41.6\left[\mathrm{C}_{\delta}(\mathrm{Arg})\right], 43.6\left[\mathrm{CH}_{2}(\mathrm{Pbf})\right], 46.0\left(\mathrm{C}_{6}\right), 50.2$ [1$\mathrm{CH}_{2}(\mathrm{Bn})$ ], $51.7\left(4-\mathrm{CH}_{2}\right), 53.6\left(\mathrm{C}_{5}-\mathrm{CH}\right), 55.7\left(\mathrm{C}_{5}\right), 65.3\left(\mathrm{C}_{3}\right), 78.8$ [C (Boc)], 81.5 [C ( $\left.\left.{ }^{\mathrm{t}} \mathrm{Bu}\right)\right]$, 86.9, 117.4, 125.3 [3C (Pbf)], 126.7, 128.1, 128.7, 128.8, 129.4, 130.2 [10CH (Ar)], 132.8,

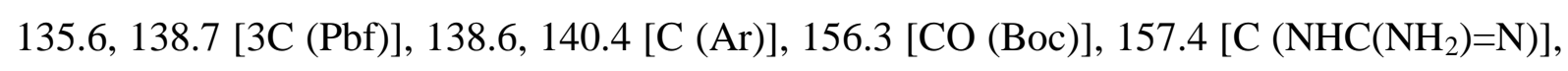
158.9 [C (Pbf)], $170.6\left(\mathrm{C}_{2}\right), 170.9\left(\mathrm{CO}_{2}\right)$; ES-MS m/z $876.1[\mathrm{M}+1]^{+}$; Anal. calcd. for $\mathrm{C}_{47} \mathrm{H}_{66} \mathrm{~N}_{6} \mathrm{O}_{8} \mathrm{~S}: \mathrm{C}, 64.51 ; \mathrm{H}, 7.60 ; \mathrm{N}, 9.60$. Found: C, 64.25; H, 7.38; N, 9.94.

4.6.14. (5S,3S)-1-Benzyl- 5-((S)-1-(tert-butoxycarbonyl)amino-2-phenylethyl)- 4-(tertbutoxycarbonyl)methyl -3-(3-(2-((2,2,4,6,7-pentamethyl-2,3-dihydrobenzofuran-5yl)sulfonyl)guanidino) propyl)-2-oxopiperazine [(S)-23a]

Amorphous solid (149 mg, 50\%); $[\alpha]_{\mathrm{D}}{ }^{20}+16\left(c\right.$ 1.0, $\left.\mathrm{CH}_{2} \mathrm{Cl}_{2}\right)$; HPLC [Sunfire $\mathrm{C}_{18}(4.6 \times$ $150 \mathrm{~mm}, 3.5 \mu \mathrm{m}), 80-100 \%$ gradient of solvent $\mathrm{A}$ in $\mathrm{B}, 30 \mathrm{~min}] t_{\mathrm{R}} 6.80 \mathrm{~min} ;{ }^{1} \mathrm{H}$ NMR (400 $\left.\mathrm{MHz},\left(\mathrm{CD}_{3}\right)_{2} \mathrm{CO}\right) \delta(\mathrm{ppm}): 1.25$ (s, 9H, Boc), 1.43 [s, 6H, 2CH $\left.(\mathrm{Pbf})\right], 1.46\left(\mathrm{~s}, 9 \mathrm{H},{ }^{\mathrm{t}} \mathrm{Bu}\right), 1.75$ $[\mathrm{m}, 4 \mathrm{H}, \beta-\mathrm{H}(\mathrm{Arg})$ and $\gamma-\mathrm{H}(\mathrm{Arg})], 2.05\left[\mathrm{~s}, 3 \mathrm{H}, \mathrm{CH}_{3}(\mathrm{Pbf})\right], 2.50\left[\mathrm{~s}, 3 \mathrm{H}, \mathrm{CH}_{3}(\mathrm{Pbf})\right], 2.57[\mathrm{~s}$, $\left.3 \mathrm{H}, \mathrm{CH}_{3}(\mathrm{Pbf})\right], 2.55$ (m, 1H, $\left.\mathrm{CH}_{2}-\mathrm{Ph}\right), 2.98$ [s, 2H, $\left.\mathrm{CH}_{2}(\mathrm{Pbf})\right], 3.03$ (d, $1 \mathrm{H}, J=13 \mathrm{~Hz}, \mathrm{CH}_{2^{-}}$ 
Ph), $3.22(\mathrm{~m}, 1 \mathrm{H}, 5-\mathrm{H}), 3.24$ [m, 2H, $\delta-\mathrm{H}(\mathrm{Arg})], 3.40(\mathrm{~m}, 2 \mathrm{H}, 6-\mathrm{H}), 3.46(\mathrm{~m}, 1 \mathrm{H}, 3-\mathrm{H}), 3.53$ $\left(\mathrm{d}, 1 \mathrm{H}, J=18 \mathrm{~Hz}, 4-\mathrm{CH}_{2}\right), 3.67\left(\mathrm{~d}, 1 \mathrm{H}, J=18 \mathrm{~Hz}, 4-\mathrm{CH}_{2}\right), 3.82(\mathrm{~m}, 1 \mathrm{H}, 5-\mathrm{CH}), 4.53[\mathrm{~d}, 1 \mathrm{H}, J$ $\left.=14.5 \mathrm{~Hz}, 1-\mathrm{CH}_{2}(\mathrm{Bn})\right], 4.70\left[\mathrm{~d}, 1 \mathrm{H}, J=14.5 \mathrm{~Hz}, 1-\mathrm{CH}_{2}(\mathrm{Bn})\right], 6.10(\mathrm{~d}, 1 \mathrm{H}, J=8 \mathrm{~Hz}$, NHBoc), $6.50\left[\mathrm{~m}, 3 \mathrm{H}, \mathrm{NHC}\left(\mathrm{NH}_{2}\right)=\mathrm{N}\right], 7.10-7.40(\mathrm{~m}, 10 \mathrm{H}, \mathrm{Ar}) ;{ }^{13} \mathrm{C} \mathrm{NMR}(100 \mathrm{MHz}$, $\left.\left(\mathrm{CD}_{3}\right)_{2} \mathrm{CO}\right) \delta(\mathrm{ppm}): 12.6,18.2,19.5\left[3 \mathrm{CH}_{3}(\mathrm{Pbf})\right], 26.4\left[\mathrm{C}_{\gamma}(\mathrm{Arg})\right], 28.3\left[3 \mathrm{CH}_{3}\left({ }^{\mathrm{t}} \mathrm{Bu}\right)\right], 28.5$ [2 $\left.\mathrm{CH}_{3}(\mathrm{Pbf})\right], 28.7$ [3 $\left.\mathrm{CH}_{3}(\mathrm{Boc})\right], 32.2\left[\mathrm{C}_{\beta}(\mathrm{Arg})\right], 35.5\left(\mathrm{CH}_{2} \mathrm{Ph}\right), 41.6\left[\mathrm{C}_{\delta}(\mathrm{Arg})\right], 43.6\left[\mathrm{CH}_{2}\right.$ (Pbf)], $44.9\left(\mathrm{C}_{6}\right), 49.9$ [1- $\left.\mathrm{CH}_{2}(\mathrm{Bn})\right], 54.0\left(\mathrm{C}_{5}-\mathrm{CH}\right), 56.4\left(4-\mathrm{CH}_{2}\right), 60.7\left(\mathrm{C}_{5}\right), 65.0\left(\mathrm{C}_{3}\right), 78.8$ [C (Boc)], 81.7 [C ('Bu)], 86.9, 117.4, 125.3 [3C (Pbf)], 126.8, 128.1, 128.8, 129.0, 129.4, 129.9 [10CH (Ar)], 132.8, 135.7, 138.7 [3C (Pbf)], 138.5, 140.6 [C (Ar)], 156.1 [CO (Boc)], $157.3\left[\mathrm{C}\left(\mathrm{NHC}\left(\mathrm{NH}_{2}\right)=\mathrm{N}\right)\right.$ ], 158.9 [C $\left.(\mathrm{Pbf})\right], 171.1\left(\mathrm{C}_{2}\right), 171.3\left(\mathrm{CO}_{2}\right)$; ES-MS m/z 876.1 $[\mathrm{M}+1]^{+}$; Anal. calcd. for $\mathrm{C}_{47} \mathrm{H}_{66} \mathrm{~N}_{6} \mathrm{O}_{8} \mathrm{~S}: \mathrm{C}, 64.51 ; \mathrm{H}, 7.60 ; \mathrm{N}, 9.60$. Found: C, 64.68; H, 7.42; N, 9.72 .

4.6.15. (5R,3S)-1-Benzyl- 5-((S)-1-(tert-butoxycarbonyl)amino-2-phenylethyl)- 4-(tertbutoxycarbonyl)methyl -3-(3-(3-benzyl-2-((2,2,4,6,7-pentamethyl-2,3dihydrobenzofuran-5-yl)sulfonyl)guanidino) propyl)-2-oxopiperazine [(R)-25a]

Amorphous solid (5:1 isomeric mixture, $46 \mathrm{mg}, 14 \%)$; HPLC [Sunfire $\mathrm{C}_{18}(4.6 \times 150 \mathrm{~mm}$, $3.5 \mu \mathrm{m}$ ), 80-100\% gradient of solvent $\mathrm{A}$ in $\mathrm{B}, 30 \mathrm{~min}] t_{\mathrm{R}} 12.82 \mathrm{~min}$ (major isomer) and 12.53 $\min$ (minor isomer); ${ }^{1} \mathrm{H} \mathrm{NMR}\left(400 \mathrm{MHz},\left(\mathrm{CD}_{3}\right)_{2} \mathrm{CO}\right) \delta(\mathrm{ppm})$ Major isomer: $1.20(\mathrm{~s}, 9 \mathrm{H}$, Boc), 1.44 [s, 6H, 2CH 3 (Pbf)], 1.45 (s, 9H, $\left.{ }^{\mathrm{t}} \mathrm{Bu}\right), 1.85$ [m, 4H, $\beta-\mathrm{H}$ (Arg) and $\left.\gamma-\mathrm{H}(\mathrm{Arg})\right], 2.01$ [s, 3H, $\left.\mathrm{CH}_{3}(\mathrm{Pbf})\right], 2.44$ [s, 3H, $\mathrm{CH}_{3}$ (Pbf)], 2.50 [s, 3H, $\mathrm{CH}_{3}(\mathrm{Pbf})$ ], 2.64 (dd, 1H, $J=11$ and $\left.14 \mathrm{~Hz}, \mathrm{CH}_{2}-\mathrm{Ph}\right), 2.93$ [s, 2H, $\left.\mathrm{CH}_{2}(\mathrm{Pbf})\right], 3.14$ (d, $\left.1 \mathrm{H}, J=16 \mathrm{~Hz}, 4-\mathrm{CH}_{2}\right), 3.26$ (m, 1H, 6-H), $3.36[\mathrm{~m}, 2 \mathrm{H}, \delta-\mathrm{H}(\mathrm{Arg})$ and $3-\mathrm{H}], 3.40(\mathrm{~m}, 1 \mathrm{H}, 5-\mathrm{H}), 3.42(\mathrm{~m}, 1 \mathrm{H}, 6-\mathrm{H}), 3.47[\mathrm{~m}, 1 \mathrm{H}, \delta-\mathrm{H}$ (Arg)], $3.48\left(\mathrm{dd}, 1 \mathrm{H}, J=3\right.$ and $\left.14 \mathrm{~Hz}, \mathrm{CH}_{2} \mathrm{Ph}\right), 3.62\left(\mathrm{~d}, 1 \mathrm{H}, J=16 \mathrm{~Hz}, 4-\mathrm{CH}_{2}\right), 3.82(\mathrm{~m}, 1 \mathrm{H}$, 5-CH), $4.36\left[\mathrm{~d}, 1 \mathrm{H}, J=14.5 \mathrm{~Hz}, 1-\mathrm{CH}_{2}(\mathrm{Bn})\right], 4.43\left[\mathrm{dd}, 1 \mathrm{H}, J=6\right.$ and $16 \mathrm{~Hz}, \mathrm{CH}_{2}$ $(\mathrm{NHC}(\mathrm{NHBn})=\mathrm{N})], 4.46\left[\mathrm{dd}, 1 \mathrm{H}, J=6\right.$ and $\left.16 \mathrm{~Hz}, \mathrm{CH}_{2}(\mathrm{NHC}(\mathrm{NHBn})=\mathrm{N})\right], 4.83[\mathrm{~d}, 1 \mathrm{H}, J=$ $\left.14.5 \mathrm{~Hz}, 1-\mathrm{CH}_{2}(\mathrm{Bn})\right], 5.86(\mathrm{~d}, 1 \mathrm{H}, J=9.5 \mathrm{~Hz}, N H \mathrm{Boc}), 7.09-7.39(\mathrm{~m}, 17 \mathrm{H}$, Ar and $N H C(N H B n)=\mathrm{N})$. Minor isomer: $1.22(\mathrm{~s}, 9 \mathrm{H}, \mathrm{Boc}), 1.43$ [s, 6H, 2CH $(\mathrm{Pbf})], 1.45(\mathrm{~s}, 9 \mathrm{H}$, $\left.{ }^{\mathrm{t}} \mathrm{Bu}\right), 1.85[\mathrm{~m}, 4 \mathrm{H}, \beta-\mathrm{H}(\mathrm{Arg})$ and $\gamma-\mathrm{H}(\mathrm{Arg})], 2.01$ [s, 3H, $\left.\mathrm{CH}_{3}(\mathrm{Pbf})\right], 2.45$ [s, 3H, $\left.\mathrm{CH}_{3}(\mathrm{Pbf})\right]$, $2.50\left[\mathrm{~s}, 3 \mathrm{H}, \mathrm{CH}_{3}(\mathrm{Pbf})\right], 2.58\left(\mathrm{~m}, 1 \mathrm{H}, \mathrm{CH}_{2} \mathrm{Ph}\right), 2.95$ [s, 2H, $\left.\mathrm{CH}_{2}(\mathrm{Pbf})\right], 3.16(\mathrm{~d}, 1 \mathrm{H}, J=16$ $\left.\mathrm{Hz}, 4-\mathrm{CH}_{2}\right), 3.26(\mathrm{~m}, 1 \mathrm{H}, 6-\mathrm{H}), 3.36[\mathrm{~m}, 2 \mathrm{H}, \delta-\mathrm{H}(\mathrm{Arg})$ and 3-H], $3.42(\mathrm{~m}, 1 \mathrm{H}, 6-\mathrm{H}), 3.47$ [m, 1H, $\delta$-H (Arg) ], $3.46\left(\mathrm{~m}, 1 \mathrm{H}, \mathrm{CH}_{2}-\mathrm{Ph}\right), 3.63\left(\mathrm{~d}, 1 \mathrm{H}, J=16 \mathrm{~Hz}, 4-\mathrm{CH}_{2}\right), 3.82(\mathrm{~m}, 1 \mathrm{H}, 5-\mathrm{CH})$, $4.36\left[\mathrm{~d}, 1 \mathrm{H}, J=14.5 \mathrm{~Hz}, 1-\mathrm{CH}_{2}(\mathrm{Bn})\right], 4.67\left[\mathrm{~d}, 1 \mathrm{H}, J=16 \mathrm{~Hz}, \mathrm{CH}_{2}(\mathrm{NHC}(\mathrm{NHBn})=\mathrm{N})\right], 4.75$ 
$\left[\mathrm{d}, 1 \mathrm{H}, J=16 \mathrm{~Hz}, \mathrm{CH}_{2}(\mathrm{NHC}(\mathrm{NHBn})=\mathrm{N})\right], 4.85\left[\mathrm{~d}, 1 \mathrm{H}, J=14.5 \mathrm{~Hz}, 1-\mathrm{CH}_{2}(\mathrm{Bn})\right], 5.85(\mathrm{~d}$, $1 \mathrm{H}, J=9.5 \mathrm{~Hz}, N H \mathrm{Boc}), 7.09-7.39(\mathrm{~m}, 17 \mathrm{H}, \mathrm{Ar}$ and $N H \mathrm{C}(N H \mathrm{Bn})=\mathrm{N}) ;{ }^{13} \mathrm{C} \mathrm{NMR}(100 \mathrm{MHz}$, $\left.\left(\mathrm{CD}_{3}\right)_{2} \mathrm{CO}\right) \delta(\mathrm{ppm})$ Major isomer: 11.9, 17.6, $18.9\left[3 \mathrm{CH}_{3}(\mathrm{Pbf})\right], 26.6\left[\mathrm{C}_{\gamma}(\mathrm{Arg})\right], 27.6\left[3 \mathrm{CH}_{3}\right.$ $\left.\left({ }^{\mathrm{t}} \mathrm{Bu}\right)\right], 27.8\left[3 \mathrm{CH}_{3}(\mathrm{Boc})\right], 28.0$ [2 $\mathrm{CH}_{3}(\mathrm{Pbf})$ and $\left.\mathrm{C}_{\beta}(\mathrm{Arg})\right], 37.4\left(\mathrm{CH}_{2} \mathrm{Ph}\right), 41.2\left[\mathrm{C}_{\delta}(\mathrm{Arg})\right]$, $42.9\left[\mathrm{CH}_{2}(\mathrm{Pbf})\right], 44.7\left[\mathrm{CH}_{2}(\mathrm{NHC}(\mathrm{NHBn})=\mathrm{N})\right], 45.4\left(\mathrm{C}_{6}\right), 49.6\left[1-\mathrm{CH}_{2}(\mathrm{Bn})\right], 50.9\left(4-\mathrm{CH}_{2}\right)$, $52.6\left(\mathrm{C}_{5}-\mathrm{CH}\right), 55.0\left(\mathrm{C}_{5}\right), 64.4\left(\mathrm{C}_{3}\right), 78.1$ [C (Boc)], 80.9 [C ( $\left.\left.{ }^{\mathrm{t}} \mathrm{Bu}\right)\right], 86.3,116.8,124.6$ [3C (Pbf)], 126.1, 127.5, 128.6, 128.2, 128.8, 129.6 [15CH (Ar)], 132.1, 134.8 [2C (Pbf)], 137.9 [2C (Ar)], 138.0 [C (Pbf)], 139.7 [C (Ar)], 153.9 [C (NHC(NHBn)=N)], 155.9 [CO (Boc)], $158.3[\mathrm{C}(\mathrm{Pbf})], 170.1\left(\mathrm{C}_{2}\right), 170.4\left(\mathrm{CO}_{2}\right)$. Minor isomer: 11.9, 17.6, 18.9 [3 $\left.\mathrm{CH}_{3}(\mathrm{Pbf})\right], 26.6$ $\left[\mathrm{C}_{\gamma}(\mathrm{Arg})\right], 27.6\left[3 \mathrm{CH}_{3}\left({ }^{\mathrm{t}} \mathrm{Bu}\right)\right], 27.8\left[3 \mathrm{CH}_{3}(\mathrm{Boc})\right], 28.0\left[2 \mathrm{CH}_{3}(\mathrm{Pbf})\right.$ and $\left.\mathrm{C}_{\beta}(\mathrm{Arg})\right], 37.4\left(\mathrm{CH}_{2^{-}}\right.$ $\mathrm{Ph}), 41.2\left[\mathrm{C}_{\delta}(\mathrm{Arg})\right], 42.9\left[\mathrm{CH}_{2}(\mathrm{Pbf})\right], 45.4\left(\mathrm{C}_{6}\right), 49.6$ [1- $\left.\mathrm{CH}_{2}(\mathrm{Bn})\right], 50.7\left(4-\mathrm{CH}_{2}\right), 50.9\left[\mathrm{CH}_{2}\right.$ $(\mathrm{NHC}(\mathrm{NHBn})=\mathrm{N})], 52.6\left(\mathrm{C}_{5}-\mathrm{CH}\right), 64.4\left(\mathrm{C}_{3}\right), 78.1$ [C $\left.(\mathrm{Boc})\right], 80.9$ [C ( $\left.{ }^{\mathrm{t}} \mathrm{Bu}\right)$ ], 86.3, 116.8, 124.6 [3C (Pbf)], 126.1, 127.5, 127.9, 128.2, 128.5, 129.5 [15CH (Ar)], 132.0, 134.8 [2C (Pbf)], 137.9 [2C (Ar)], 138.0 [C (Pbf)], 139.7 [C (Ar)], 153.9 [C (NHC(NHBn)=N)], 155.9 [CO (Boc)], 158.2 [C (Pbf)], $169.9\left(\mathrm{C}_{2}\right), 170.4\left(\mathrm{CO}_{2}\right)$; ES-MS m/z $966.2[\mathrm{M}+1]^{+}$; Anal. calcd. for $\mathrm{C}_{54} \mathrm{H}_{72} \mathrm{~N}_{6} \mathrm{O}_{8} \mathrm{~S}$ : C, 67.19; H, 7.52; N, 8.71. Found: C, 67.45; H, 7.38; N, 9.02.

4.6.16. (5S,3S)-1-Benzyl- 5-((S)-1-(tert-butoxycarbonyl)amino-2-phenylethyl)- 4-(tertbutoxycarbonyl)methyl -3-(3-(3-benzyl-2-((2,2,4,6,7-pentamethyl-2,3dihydrobenzofuran-5-yl)sulfonyl)guanidino) propyl)-2-oxopiperazine [(S)-25a]

Amorphous solid (3:1 isomeric mixture, $49 \mathrm{mg}, 15 \%)$; HPLC [Sunfire $\mathrm{C}_{18}(4.6 \times 150 \mathrm{~mm}$, $3.5 \mu \mathrm{m}$ ), 80-100\% gradient of solvent $\mathrm{A}$ in $\mathrm{B}, 30 \mathrm{~min}$ ] $t_{\mathrm{R}} 12.28 \mathrm{~min}$ (major isomer) and 12.83 min (minor isomer); ${ }^{1} \mathrm{H}$ NMR $\left(400 \mathrm{MHz},\left(\mathrm{CD}_{3}\right)_{2} \mathrm{CO}\right) \delta(\mathrm{ppm})$ Major isomer: $1.25(\mathrm{~s}, 9 \mathrm{H}$, Boc), 1.45 [s, 6H, 2CH 3 (Pbf)], $1.46\left(\mathrm{~s}, 9 \mathrm{H},{ }^{\mathrm{t}} \mathrm{Bu}\right), 1.77$ [m, 4H, $\beta-\mathrm{H}(\mathrm{Arg})$ and $\left.\gamma-\mathrm{H}(\mathrm{Arg})\right], 2.02$ [s, 3H, $\mathrm{CH}_{3}$ (Pbf)], 2.44 [s, 3H, $\left.\mathrm{CH}_{3}(\mathrm{Pbf})\right], 2.52$ [s, 3H, $\left.\mathrm{CH}_{3}(\mathrm{Pbf})\right], 2.54$ (m, 1H, CH $\left.2-\mathrm{Ph}\right)$, 2.95 [s, 2H, $\mathrm{CH}_{2}$ (Pbf)], 3.03 (m, 1H, $\left.\mathrm{CH}_{2}-\mathrm{Ph}\right), 3.24$ (m, 1H, 5-H), 3.30 [m, 2H, $\delta-\mathrm{H}$ (Arg)], $3.40(\mathrm{~m}, 2 \mathrm{H}, 6-\mathrm{H}), 3.51(\mathrm{~m}, 1 \mathrm{H}, 3-\mathrm{H}), 3.54\left(\mathrm{~m}, 1 \mathrm{H}, 4-\mathrm{CH}_{2}\right), 3.68\left(\mathrm{~m}, 1 \mathrm{H}, 4-\mathrm{CH}_{2}\right), 3.88(\mathrm{~m}$, $1 \mathrm{H}, 5-\mathrm{CH}), 4.44\left[\mathrm{~d}, 1 \mathrm{H}, J=5.5 \mathrm{~Hz}, \mathrm{CH}_{2}(\mathrm{NHC}(\mathrm{NHBn})=\mathrm{N})\right], 4.52\left[\mathrm{~d}, 1 \mathrm{H}, J=15 \mathrm{~Hz}, 1-\mathrm{CH}_{2}\right.$ (Bn)], 4.70 [d, 1H, $\left.J=15 \mathrm{~Hz}, 1-\mathrm{CH}_{2}(\mathrm{Bn})\right], 6.08$ (d, 1H, $J=8.5 \mathrm{~Hz}, N H \mathrm{Boc}$ ), 7.10-7.37 (m, $17 \mathrm{H}, \mathrm{Ar}$ and $N H C(N H \mathrm{Bn})=\mathrm{N})$. Minor isomer: $1.24(\mathrm{~s}, 9 \mathrm{H}, \mathrm{Boc}), 1.44\left[\mathrm{~s}, 6 \mathrm{H}, 2 \mathrm{CH}_{3}(\mathrm{Pbf})\right]$, $1.45\left(\mathrm{~s}, 9 \mathrm{H},{ }^{\mathrm{t}} \mathrm{Bu}\right), 1.77[\mathrm{~m}, 4 \mathrm{H}, \beta-\mathrm{H}(\mathrm{Arg})$ and $\gamma-\mathrm{H}(\mathrm{Arg})], 2.02\left[\mathrm{~s}, 3 \mathrm{H}, \mathrm{CH}_{3}(\mathrm{Pbf})\right], 2.47[\mathrm{~s}, 3 \mathrm{H}$, $\mathrm{CH}_{3}$ (Pbf)], 2.52 [s, 3H, CH $(\mathrm{Pbf})$ ], 2.54 (m, 1H, $\left.\mathrm{CH}_{2}-\mathrm{Ph}\right), 2.97$ [s, 2H, $\mathrm{CH}_{2}$ (Pbf)], 3.03 (m, 1H, $\left.\mathrm{CH}_{2}-\mathrm{Ph}\right), 3.24(\mathrm{~m}, 1 \mathrm{H}, 5-\mathrm{H}), 3.30$ [m, 2H, $\left.\delta-\mathrm{H}(\mathrm{Arg})\right], 3.40(\mathrm{~m}, 2 \mathrm{H}, 6-\mathrm{H}), 3.51(\mathrm{~m}, 1 \mathrm{H}, 3-$ 
$\mathrm{H}), 3.59\left(\mathrm{~m}, 1 \mathrm{H}, 4-\mathrm{CH}_{2}\right), 3.63\left(\mathrm{~m}, 1 \mathrm{H}, 4-\mathrm{CH}_{2}\right), 3.88(\mathrm{~m}, 1 \mathrm{H}, 5-\mathrm{CH}), 4.48\left[\mathrm{~m}, 1 \mathrm{H}, \mathrm{CH}_{2}\right.$ $(\mathrm{NHC}(\mathrm{NHBn})=\mathrm{N})], 4.58\left[\mathrm{~m}, 1 \mathrm{H}, 1-\mathrm{CH}_{2}(\mathrm{Bn})\right], 4.64\left[\mathrm{~m}, 1 \mathrm{H}, 1-\mathrm{CH}_{2}(\mathrm{Bn})\right], 6.08(\mathrm{~d}, 1 \mathrm{H}, J=8.5$ $\mathrm{Hz}, N H \mathrm{Boc}), 7.10-7.37$ [m, 17H, Ar and $N H \mathrm{C}(N H \mathrm{Bn})=\mathrm{N}] ;{ }^{13} \mathrm{C} \mathrm{NMR}\left(100 \mathrm{MHz},\left(\mathrm{CD}_{3}\right)_{2} \mathrm{CO}\right) \delta$ (ppm) Major isomer: 12.6, 18.2, 19.5 [3 $\left.\mathrm{CH}_{3}(\mathrm{Pbf})\right], 26.3\left[\mathrm{C}_{\gamma}(\mathrm{Arg})\right], 28.4\left[3 \mathrm{CH}_{3}(\mathrm{Boc})\right], 28.5$ $\left[2 \mathrm{CH}_{3}(\mathrm{Pbf})\right], 28.8\left[3 \mathrm{CH}_{3}\left({ }^{\mathrm{t}} \mathrm{Bu}\right)\right], 32.1\left[\mathrm{C}_{\beta}(\mathrm{Arg})\right], 35.3\left(\mathrm{CH}_{2}-\mathrm{Ph}\right), 42.0\left[\mathrm{C}_{\delta}(\mathrm{Arg})\right], 43.7\left[\mathrm{CH}_{2}\right.$ $(\mathrm{Pbf})], 44.9\left[\mathrm{C}_{6}\right.$ and $\left.\mathrm{CH}_{2}(\mathrm{NHC}(\mathrm{NHBn})=\mathrm{N})\right], 50.0\left[1-\mathrm{CH}_{2}(\mathrm{Bn})\right], 53.9\left(\mathrm{C}_{5}-\mathrm{CH}\right), 56.2\left(4-\mathrm{CH}_{2}\right)$, $60.7\left(\mathrm{C}_{5}\right), 64.8\left(\mathrm{C}_{3}\right), 78.1[\mathrm{C}(\mathrm{Boc})], 81.7$ [C ( $\left.\left.{ }^{\mathrm{t}} \mathrm{Bu}\right)\right], 86.9,117.5,125.3$ [3C (Pbf)], 126.8, 128.1, 128.7, 129.0, 129.4, 129.9 [15CH (Ar)], 132.8, 135.6 [2C (Pbf)], 138.4 [2C (Ar)], $138.7[\mathrm{C}(\mathrm{Pbf})], 140.7$ [C (Ar)], 156.0 [CO (Boc) and C (NHC(NHBn)=N)], 158.9 [C (Pbf)], $171.1\left(\mathrm{C}_{2}\right), 171.2\left(\mathrm{CO}_{2}\right)$. Minor isomer: 12.6, 18.2, $19.5\left[3 \mathrm{CH}_{3}(\mathrm{Pbf})\right], 26.3\left[\mathrm{C}_{\gamma}(\mathrm{Arg})\right], 28.4$ [3 $\left.3 \mathrm{CH}_{3}(\mathrm{Boc})\right], 28.5\left[2 \mathrm{CH}_{3}(\mathrm{Pbf})\right], 28.8\left[3 \mathrm{CH}_{3}\left({ }^{\mathrm{t}} \mathrm{Bu}\right)\right], 32.1\left[\mathrm{C}_{\beta}(\mathrm{Arg})\right], 35.3\left(\mathrm{CH}_{2}-\mathrm{Ph}\right), 42.0\left[\mathrm{C}_{\delta}\right.$ $(\mathrm{Arg})], 43.7\left[\mathrm{CH}_{2}(\mathrm{Pbf})\right], 44.9\left(\mathrm{C}_{6}\right), 50.0\left[1-\mathrm{CH}_{2}(\mathrm{Bn})\right], 52.6\left[\mathrm{CH}_{2}(\mathrm{NHC}(\mathrm{NHBn})=\mathrm{N})\right], 53.9$ $\left(\mathrm{C}_{5}-\mathrm{CH}\right), 56.2\left(4-\mathrm{CH}_{2}\right), 60.7\left(\mathrm{C}_{5}\right), 64.8\left(\mathrm{C}_{3}\right), 78.7$ [C $\left.(\mathrm{Boc})\right], 81.7$ [C $\left.\left({ }^{\mathrm{t}} \mathrm{Bu}\right)\right], 86.9,117.5,125.3$ [3C (Pbf)], 126.8, 128.1, 128.9, 129.2, 129.4, 129.9 [15CH (Ar)], 132.7, 135.6 [2C (Pbf)], $138.4[2 \mathrm{C} \quad(\mathrm{Ar})], \quad 138.7$ [C $(\mathrm{Pbf})], \quad 140.7$ [C $(\mathrm{Ar})], \quad 156.0$ [CO (Boc)], 156.1 [C $(\mathrm{NHC}(\mathrm{NHBn})=\mathrm{N})], 158.9$ [C $(\mathrm{Pbf})], 171.1\left(\mathrm{C}_{2}\right), 171.2\left(\mathrm{CO}_{2}\right)$; ES-MS m/z $966.2[\mathrm{M}+1]^{+}$; Anal. Calcd. for $\mathrm{C}_{54} \mathrm{H}_{72} \mathrm{~N}_{6} \mathrm{O}_{8} \mathrm{~S}$ : C, 67.19; H, 7.52; N, 8.71. Found: C, 67.37; H, 7.74; N, 8.84.

4.7. General procedure for the benzylation of the 4-alkyl-2-oxopiperazines $\mathbf{1 0 b}$ and $\mathbf{1 2 b}$. Synthesis of the 4-alkyl-1-benzyl-2-oxopiperazines (R)- and (S)-(26b and $27 \boldsymbol{b})$

$\mathrm{NaH}(60 \%$ suspension in mineral oil, $9 \mathrm{mg}, 0.38 \mathrm{mmol})$ and benzyl bromide $(0.45 \mu \mathrm{L}, 0.38$ mmol) were added to a solution of the corresponding 2-oxopiperazine $\mathbf{1 0 b}$ and $\mathbf{1 2 b}(0.34$ mmol) in anhydrous mixture THF/DMF $(9: 1,10 \mathrm{~mL})$ under argon at $0^{\circ} \mathrm{C}$. After $1 \mathrm{~h}$ of stirring, the crude reaction mixture was diluted with EtOAc $(100 \mathrm{~mL})$ and the excess of $\mathrm{NaH}$ was hydrolysed by addition of $\mathrm{H}_{2} \mathrm{O}(20 \mathrm{~mL})$. The aqueous layer was extracted with EtOAc $(2 \times 50$ $\mathrm{mL}$ ) and the organic extracts were dried over $\mathrm{Na}_{2} \mathrm{SO}_{4}$ and evaporated to dryness. The residue was purified by flash chromatography, using $\mathrm{MeOH}$ in $\mathrm{CH}_{2} \mathrm{Cl}_{2}$ gradient as mobile phase to obtain the respective 4-alkyl-1-benzyl-2-oxopiperazine derivatives $(\boldsymbol{R})$ - and $(\boldsymbol{S})-(\mathbf{2 6} \mathbf{b}$ and 27b) in 70-80 \%. The compounds were dissolved in $\mathrm{CH}_{3} \mathrm{CN} / \mathrm{H}_{2} \mathrm{O}(1: 2,2 \mathrm{~mL})$ and the solutions were lyophilized. 
4.7.1. (5R,3S)-1,4-Dibenzyl-5-((S)-1-((tert-butoxycarbonyl)amino-2-(1-(tert-butoxycarbonyl)-indol-3-yl)ethyl-3-(4-((tert-butoxycarbonyl)amino)butyl)-2-oxopiperazine [(R)-26b]

Amorphous solid (220 mg, 80\%); $[\alpha]_{\mathrm{D}}^{20}-15\left(c 1.4, \mathrm{CH}_{2} \mathrm{Cl}_{2}\right)$; HPLC [Sunfire $\mathrm{C}_{18}(4.6 \times 150$ $\mathrm{mm}, 3.5 \mu \mathrm{m}), 80-100 \%$ gradient of solvent $\mathrm{A}$ in $\mathrm{B}, 30 \mathrm{~min}] t_{\mathrm{R}} 12.75 \mathrm{~min} ;{ }^{1} \mathrm{H} \mathrm{NMR}(400 \mathrm{MHz}$, $\left.\left(\mathrm{CD}_{3}\right)_{2} \mathrm{CO}\right) \delta(\mathrm{ppm}): 1.25$ (s, 9H, Boc), 1.31 [m, 4H, $\gamma-\mathrm{H}$ (Lys) and $\delta-\mathrm{H}$ (Lys)], 1.36 [s, 9H, Boc (Lys)], 1.61 [m, 1H, $\beta$-H (Lys)], 1.64 [s, 9H, Boc (Ind)], 1.80 [m, 1H, $\beta$-H (Lys)], 1.86 [m, 1H, $\varepsilon-\mathrm{H}$ (Lys)], 2.80 (m, 1H, $C_{2}$-Ind), 3.03 [m, 1H, $\varepsilon-\mathrm{H}$ (Lys)], 3.17 (dd, 1H, $J=4.5$ and $10 \mathrm{~Hz}, 3-\mathrm{H}), 3.37$ (m, 1H, 6-H), 3.54 [m, 1H, 4- $\left.\mathrm{CH}_{2}(\mathrm{Bn})\right], 3.59$ (m, 3H, 5-H, 6-H and $\mathrm{CH}_{2^{-}}$ Ind), $4.17(\mathrm{~m}, 1 \mathrm{H}, 5-\mathrm{CH}), 4.18\left[\mathrm{~d}, 1 \mathrm{H}, J=13.5 \mathrm{~Hz}, 4-\mathrm{CH}_{2}(\mathrm{Bn})\right], 4.51[\mathrm{~d}, 1 \mathrm{H}, J=14.5 \mathrm{~Hz}, 1-$ $\left.\mathrm{CH}_{2}(\mathrm{Bn})\right], 4.79$ [d, $\left.1 \mathrm{H}, J=14.5 \mathrm{~Hz}, 1-\mathrm{CH}_{2}(\mathrm{Bn})\right], 5.84$ [m, 1H, NHBoc (Lys)], 6.00 (d, 1H, $J$ $=9.5 \mathrm{~Hz}, N H \mathrm{Boc}), 7.16(\mathrm{t}, 1 \mathrm{H}, J=7.5 \mathrm{~Hz}, \mathrm{Ar}), 7.22-7.57(\mathrm{~m}, 13 \mathrm{H}, \mathrm{Ar}), 8.12(\mathrm{~d}, 1 \mathrm{H}, J=7.5$ $\mathrm{Hz}, \mathrm{Ar}) ;{ }^{13} \mathrm{C} \mathrm{NMR}\left(100 \mathrm{MHz},\left(\mathrm{CD}_{3}\right)_{2} \mathrm{CO}\right) \delta$ (ppm): $24.5\left[\mathrm{C}_{\gamma}\right.$ (Lys)], 27.9 ( $\mathrm{CH}_{2}$-Ind), 28.3, 28.5, $28.7\left[9 \mathrm{CH}_{3}(3 \mathrm{Boc})\right], 30.3\left[\mathrm{C}_{\delta}(\mathrm{Lys})\right], 31.2\left[\mathrm{C}_{\beta}(\mathrm{Lys})\right], 41.1\left[\mathrm{C}_{\varepsilon}(\mathrm{Lys})\right], 45.8\left(\mathrm{C}_{6}\right), 50.2[1-$ $\left.\mathrm{CH}_{2}(\mathrm{Bn})\right], 51.3\left(\mathrm{C}_{5}-\mathrm{CH}\right), 51.9$ [4- $\left.\mathrm{CH}_{2}(\mathrm{Bn})\right], 55.6\left(\mathrm{C}_{5}\right), 62.5\left(\mathrm{C}_{3}\right), 78.2,78.9,83.9$ [3C (3Boc)], 115.8 [CH (Ar)], 118.7 [C (Ar)], 119.9, 123.2, 124.3, 124.9, 128.1, 128.2, 128.9, 129.3, 129.4, 129.9 [14CH (Ar)], 131.9, 136.3, 138.9, 139.5 [4C (Ar)], 150.3, 156.5 [3CO (3Boc)], $171.0\left(\mathrm{C}_{2}\right)$; ES-MS $m / z, 811.2[\mathrm{M}+1]^{+}$; Anal. calcd. for $\mathrm{C}_{47} \mathrm{H}_{63} \mathrm{~N}_{5} \mathrm{O}_{7}: \mathrm{C}, 69.69 ; \mathrm{H}$, 7.84; N, 8.65. Found: C, 69.92; H, 8.06; N, 8.41.

4.7.2. (5S,3S)-1,4-Dibenzyl-5-((S)-1-((tert-butoxycarbonyl)amino-2-(1-(tert-butoxycarbonyl)-indol-3-yl)ethyl-3-(4-((tert-butoxycarbonyl)amino)butyl)-2-oxopiperazine [(S)26b]

Amorphous solid (220 mg, 80\%); $[\alpha]_{\mathrm{D}}^{20}+11\left(c\right.$ 1.3, $\left.\mathrm{CH}_{2} \mathrm{Cl}_{2}\right)$; HPLC [Sunfire $\mathrm{C}_{18}(4.6 \times$ $150 \mathrm{~mm}, 3.5 \mu \mathrm{m}), 80-100 \%$ gradient of solvent $\mathrm{A}$ in $\mathrm{B}, 30 \mathrm{~min}] t_{\mathrm{R}} 12.99 \mathrm{~min} ;{ }^{1} \mathrm{H}$ NMR (400 $\left.\mathrm{MHz},\left(\mathrm{CD}_{3}\right)_{2} \mathrm{CO}\right) \delta(\mathrm{ppm}): 1.28[\mathrm{~m}, 2 \mathrm{H}, \gamma-\mathrm{H}(\mathrm{Lys})], 1.31[\mathrm{~m}, 9 \mathrm{H}, \mathrm{Boc}], 1.34[\mathrm{~m}, 2 \mathrm{H}, \delta-\mathrm{H}$ (Lys)], 1.37 [m, 9H, Boc (Lys)], 1.43 [m, 1H, $\beta$-H (Lys)], 1.66 [s, 9H, Boc (Ind)], 1.71 [m, 1H, $\beta$-H (Lys)], 2.85 (m, 1H, $C_{2}$-Ind), 2.95 [m, 2H, $\varepsilon-\mathrm{H}$ (Lys)], 3.16 (m, 1H, 5-H), 3.19 (m, 1H, $\mathrm{CH}_{2}$-Ind), 3.27 (t, $\left.1 \mathrm{H}, J=7 \mathrm{~Hz}, 3-\mathrm{H}\right), 3.46$ (dd, $1 \mathrm{H}, J=5$ and $\left.13.5 \mathrm{~Hz}, 6-\mathrm{H}\right), 3.57$ (m, 1H, 6-H), 3.85 [d, 1H, $\left.J=14 \mathrm{~Hz}, 4-\mathrm{CH}_{2}(\mathrm{Bn})\right], 4.08$ [d, 1H, $\left.J=14 \mathrm{~Hz}, 4-\mathrm{CH}_{2}(\mathrm{Bn})\right], 4.12$ (m, 1H, 5-CH), 4.57 [d, 1H, $\left.J=15 \mathrm{~Hz}, 1-\mathrm{CH}_{2}(\mathrm{Bn})\right], 4.66$ [d, 1H, $\left.J=15 \mathrm{~Hz}, 1-\mathrm{CH}_{2}(\mathrm{Bn})\right], 5.80$ [m, $1 \mathrm{H}, N H \mathrm{Boc}(\mathrm{Lys})], 6.09$ (d, 1H, $J=9 \mathrm{~Hz}, N H \mathrm{Boc}), 7.18-7.38$ (m, 10H, Ar), 7.47 (m, 3H, Ar), 
$7.61(\mathrm{~d}, 1 \mathrm{H}, J=7.5 \mathrm{~Hz}, \mathrm{Ar}), 8.12(\mathrm{~d}, 1 \mathrm{H}, J=7.5 \mathrm{~Hz}, \mathrm{Ar}) .{ }^{13} \mathrm{C} \mathrm{NMR}\left(100 \mathrm{MHz},\left(\mathrm{CD}_{3}\right)_{2} \mathrm{CO}\right) \delta$ (ppm): $24.2\left[\mathrm{C}_{\gamma}\right.$ (Lys)], $26.4\left(\mathrm{CH}_{2}\right.$-Ind), 28.3, $28.7\left[9 \mathrm{CH}_{3}\right.$ (3Boc)], $31.2\left[\mathrm{C}_{\delta}(\mathrm{Lys})\right], 34.8\left[\mathrm{C}_{\beta}\right.$ (Lys)], $41.0\left[\mathrm{C}_{\varepsilon}(\mathrm{Lys})\right], 45.0\left[\mathrm{C}_{6}\right], 49.8$ [1- $\left.\mathrm{CH}_{2}(\mathrm{Bn})\right], 52.8\left(\mathrm{C}_{5}-\mathrm{CH}\right), 62.3$ [4- $\left.\mathrm{CH}_{2}(\mathrm{Bn})\right], 63.6$ $\left(\mathrm{C}_{5}\right), 64.3\left(\mathrm{C}_{3}\right), 78.2,78.8,84.0$ [3C (3Boc)], 115.9 [CH (Ar)], 119.0 [C (Ar)], 119.9, 123.2, 124.0, 125.0, 128.1, 128.7, 129.1, 129.4, 130.2 [14CH (Ar)], 131.8, 136.3, 138.7, 139.9 [4C (Ar)], 150.3, 156.5 [3CO (3Boc)], $171.7\left(\mathrm{C}_{2}\right)$; ES-MS m/z $811.2[\mathrm{M}+1]^{+}$; Anal. calcd. for $\mathrm{C}_{47} \mathrm{H}_{63} \mathrm{~N}_{5} \mathrm{O}_{7}$ : C, 69.69; H, 7.84; N, 8.65. Found: C, 69.90; H, 7.96; N, 8.52.

4.7.3. (5R,3S)-1-Benzyl-5-((S)-1-((tert-butoxycarbonyl)amino-2-(1-(tert-butoxycarbonyl)indol-3-yl)ethyl-4-(tert-butoxycarbonyl)methyl -3-(4-((tert-butoxycarbonyl)amino)butyl)-2-oxopiperazine [(R)-27b]

Amorphous solid (227 mg, 80\%); $[\alpha]_{\mathrm{D}}^{20}-4.2$ (c 1.5, $\left.\mathrm{CH}_{2} \mathrm{Cl}_{2}\right)$; HPLC [Sunfire $\mathrm{C}_{18}(4.6 \times$ $150 \mathrm{~mm}, 3.5 \mu \mathrm{m}), 80-100 \%$ gradient of solvent $\mathrm{A}$ in $\mathrm{B}, 30 \mathrm{~min}] t_{\mathrm{R}} 11.80 \mathrm{~min} ;{ }^{1} \mathrm{H}$ NMR (400 $\left.\mathrm{MHz},\left(\mathrm{CD}_{3}\right)_{2} \mathrm{CO}\right) \delta$ (ppm): 1.23 (s, 9H, Boc), 1.37 [s, 9H, Boc (Lys)], 1.48 (s, 9H, $\left.{ }^{\mathrm{t}} \mathrm{Bu}\right), 1.61$ [m, 2H, $\gamma$-H (Lys)], 1.63 [m, 2H, $\delta$-H (Lys)], 1.65 [s, 9H, Boc (Ind)], 1.79 [m, 1H, $\beta$-H (Lys)], $1.87\left[\mathrm{~m}, 1 \mathrm{H}, \beta-\mathrm{H}\right.$ (Lys)], 2.75 (dd, $1 \mathrm{H}, J=10.5$ and $15 \mathrm{~Hz}, \mathrm{CH}_{2}$-Ind), 3.13 (m, $1 \mathrm{H}, 4-\mathrm{CH}_{2}$ ), 3.16 [m, 2H, $\varepsilon-\mathrm{H}$ (Lys)], 3.29 (m, 1H, 6-H), 3.33 (m, 1H, 3-H), 3.40 (m, 1H, 6-H), 3.46 (m, 1H, 5-H), 3.61 (m, 1H, $\mathrm{CH}_{2}$-Ind), $3.62\left(\mathrm{~d}, 1 \mathrm{H}, J=16 \mathrm{~Hz}, 4-\mathrm{CH}_{2}\right), 3.91$ (m, 1H, 5-CH), 4.38 $\left[\mathrm{d}, 1 \mathrm{H}, J=14.5 \mathrm{~Hz}, 1-\mathrm{CH}_{2}(\mathrm{Bn})\right], 4.84\left[\mathrm{~d}, 1 \mathrm{H}, J=14.5 \mathrm{~Hz}, 1-\mathrm{CH}_{2}(\mathrm{Bn})\right], 5.93[\mathrm{~m}, 1 \mathrm{H}, N H$ Boc (Lys)], 5.98 (d, 1H, $J=9.5 \mathrm{~Hz}, N H \mathrm{Boc}), 7.22$ (t, 1H, $J=7.5 \mathrm{~Hz}, \mathrm{Ar}), 7.25-7.37$ (m, 6H, Ar), 7.47 (s, 1H, Ind), 7.62 (d, 1H, $J=7.5 \mathrm{~Hz}$, Ind), 8.11 (d, $1 \mathrm{H}, J=7.5 \mathrm{~Hz}$, Ind); ${ }^{13} \mathrm{C}$ NMR (100 $\left.\mathrm{MHz},\left(\mathrm{CD}_{3}\right)_{2} \mathrm{CO}\right) \delta$ (ppm): $24.7\left[\mathrm{C}_{\gamma}\right.$ (Lys)], $27.6\left(\mathrm{CH}_{2}\right.$-Ind), 28.3 [6 $6 \mathrm{CH}_{3}\left(\mathrm{Boc}\right.$ and $\left.\left.{ }^{\mathrm{t}} \mathrm{Bu}\right)\right], 28.5$, $28.7\left[6 \mathrm{CH}_{3}(2 \mathrm{Boc})\right], 30.4\left[\mathrm{C}_{\delta}(\mathrm{Lys})\right], 31.3\left[\mathrm{C}_{\beta}(\mathrm{Lys})\right], 41.2\left[\mathrm{C}_{\varepsilon}(\mathrm{Lys})\right], 46.0\left(\mathrm{C}_{6}\right), 50.2$ [1- $\mathrm{CH}_{2}$ $(\mathrm{Bn})], 51.9\left(\mathrm{C}_{5}-\mathrm{CH}\right), 52.1\left(4-\mathrm{CH}_{2}\right), 56.0\left(\mathrm{C}_{5}\right), 65.6\left(\mathrm{C}_{3}\right), 78.2,78.9$ [2C (2Boc)], 81.5 [C $\left.\left({ }^{\mathrm{t}} \mathrm{Bu}\right)\right], 83.9[\mathrm{C}(\mathrm{Boc})], 115.8[\mathrm{CH}(\mathrm{Ar})], 118.9$ [C (Ar)], 120.0, 123.1, 124.4, 124.9, 128.1, 128.8, 129.4 [9CH (Ar)], 131.9, 136.3, 138.7 [3C (Ar)], 150.3, 156.4 [3CO (3Boc)], 170.7 $\left[\mathrm{CO}_{2}\right], 170.8\left(\mathrm{C}_{2}\right)$; ES-MS m/z 835.1 [M+1] $]^{+}$; Anal. calcd. for $\mathrm{C}_{46} \mathrm{H}_{67} \mathrm{~N}_{5} \mathrm{O}_{9}$ : C, 66.24; $\mathrm{H}, 8.10$; N, 8.40. Found: C, 66.03; H, 7.86; N, 8.35.

4.7.4. (5S,3S)-1-Benzyl-5-((S)-1-((tert-butoxycarbonyl)amino-2-(1-(tert-butoxycarbonyl)indol-3-yl)ethyl-4-(tert-butoxycarbonyl)methyl-3-(4-((tert-butoxycarbonyl)amino)butyl)-2-oxopiperazine [(S)-27b] 
Amorphous solid (198 mg, 70\%); $[\alpha]_{\mathrm{D}}^{20}+7.0\left(c\right.$ 1.3, $\left.\mathrm{CH}_{2} \mathrm{Cl}_{2}\right)$; HPLC [Sunfire $\mathrm{C}_{18}(4.6 \times$ $150 \mathrm{~mm}, 3.5 \mu \mathrm{m}), 80-100 \%$ gradient of solvent $\mathrm{A}$ in $\mathrm{B}, 30 \mathrm{~min}] t_{\mathrm{R}} 12.41 \mathrm{~min} ;{ }^{1} \mathrm{H}$ NMR (400 $\left.\mathrm{MHz},\left(\mathrm{CD}_{3}\right)_{2} \mathrm{CO}\right) \delta(\mathrm{ppm}): 1.29$ (s, 9H, Boc), 1.37 [s, 9H, Boc (Lys)], 1.45 (s, 9H, $\left.{ }^{\mathrm{t}} \mathrm{Bu}\right), 1.55$ [m, 2H, $\gamma$-H (Lys)], 1.57 [m, 2H, $\delta$-H (Lys)], 1.65 [s, 9H, Boc (Ind)], 1.80 [m, 2H, $\beta$-H (Lys)], $2.71\left(\mathrm{dd}, 1 \mathrm{H}, \mathrm{J}=11\right.$ and $15 \mathrm{~Hz}, \mathrm{CH}_{2}$-Ind), 3.17 (dd, $1 \mathrm{H}, \mathrm{J}=2$ and $15 \mathrm{~Hz}, \mathrm{CH}_{2}$-Ind), 3.09 [m, 2H, $\varepsilon-\mathrm{H}$ (Lys)], 3.30 (m, 1H, 5-H), 3.44 (m, 1H, 3-H), 3.47 (m, 2H, 6-H), 3.58 (d, 1H, J = 18 $\left.\mathrm{Hz}, 4-\mathrm{CH}_{2}\right), 3.70$ (d, $\left.1 \mathrm{H}, J=18 \mathrm{~Hz}, 4-\mathrm{CH}_{2}\right), 4.02$ (m, 1H, 5-CH), 4.64 [m, 2H, 1- $\left.\mathrm{CH}_{2}(\mathrm{Bn})\right]$, 5.92 [m, 1H, NHBoc (Lys)], 6.14 (d, 1H, J = 8.5 Hz, NHBoc), 7.18-7.41 (m, 7H, Ar), 7.48 (s, $1 \mathrm{H}, \mathrm{Ar}), 7.61(\mathrm{~d}, 1 \mathrm{H}, J=7.5 \mathrm{~Hz}, \mathrm{Ar}), 8.11(\mathrm{~d}, 1 \mathrm{H}, J=7.5 \mathrm{~Hz}, \mathrm{Ar}) .{ }^{13} \mathrm{C}$ NMR $(100 \mathrm{MHz}$, $\left.\left(\mathrm{CD}_{3}\right)_{2} \mathrm{CO}\right) \delta(\mathrm{ppm}): 23.9$ [C $\mathrm{C}_{\gamma}$ (Lys)], $25.3\left(\mathrm{CH}_{2}\right.$-Ind), 28.3 [6 $\mathrm{CH}_{3}\left(\mathrm{Boc}\right.$ and $\left.\left.{ }^{\mathrm{t}} \mathrm{Bu}\right)\right], 28.6,28.7$

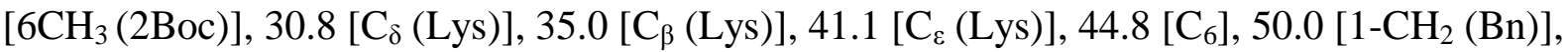
$51.9\left(\mathrm{C}_{5}-\mathrm{CH}\right), 56.9\left(4-\mathrm{CH}_{2}\right), 60.9\left(\mathrm{C}_{5}\right), 65.6\left(\mathrm{C}_{3}\right), 78.2,78.8$ [2C (2Boc) $], 81.7$ [C $\left.\left({ }^{\mathrm{t}} \mathrm{Bu}\right)\right], 84.0$ [C (Boc)], 115.9 [CH (Ar)], 119.1 [C (Ar)], 119.9, 123.3, 124.1, 125.0, 128.1, 128.8, 129.4 [9CH (Ar)], 131.7, 136.4, 138.7 [3C (Ar)], 150.3, 156.6 [3CO (3Boc)], $171.2\left(\mathrm{CO}_{2}\right), 171.3$ $\left(\mathrm{C}_{2}\right)$; ES-MS m/z 835.1 [M+1] $]^{+}$; Anal. calcd. for $\mathrm{C}_{46} \mathrm{H}_{67} \mathrm{~N}_{5} \mathrm{O}_{9}$ : C, 66.24; H, 8.10; N, 8.40. Found: C, 65.98; H, 8.36; N, 8.61.

4.8. General procedure for the synthesis of the 1,4-dibenzyl-2-oxopiperazine-derived benzyl ureas (R)- and (S)-(28a and 29a)

The corresponding 1,4-dibenzyl-2-oxopiperazine $\mathbf{1 4 a}$ and $\mathbf{1 7 a}(0.10 \mathrm{mmol})$ was dissolved in $3.4 \mathrm{~N}$ solution of $\mathrm{HCl}$ in EtOAc $(5 \mathrm{~mL})$ and the mixture was stirred at room temperature for $30 \mathrm{~min}$. Afterward, the solvent was evaporated to dryness and the residue was dissolved in $\mathrm{CH}_{3} \mathrm{CN} / \mathrm{H}_{2} \mathrm{O}(1: 3,2 \mathrm{~mL})$ and the solution was lyophilized. The residue was dissolved in dry THF $(10 \mathrm{~mL})$ and TEA $(56 \mu \mathrm{L}, 0.40 \mathrm{mmol})$ and benzyl isocyanate $(15 \mu \mathrm{L}, 0.12 \mathrm{mmol})$ were successively added to the solution. After $1 \mathrm{~h}$ of stirring at room temperature, the solvent was removed under reduced pressure and the residue was dissolved in $\mathrm{CH}_{2} \mathrm{Cl}_{2}$ (40 mL). The solution was successively washed with $\mathrm{H}_{2} \mathrm{O}(10 \mathrm{~mL})$ and brine $(10 \mathrm{~mL})$, dried over $\mathrm{Na}_{2} \mathrm{SO}_{4}$, and evaporated to dryness. The residue was purified by flash chromatography, using a $\mathrm{MeOH}$ in EtOAc gradient as mobile phase to obtain the resèctove 1,4-dibenzyl-2-oxopiperazinederived benzyl ureas 28a and 29a in 50-85\% yield. The purified compounds were dissolved in $\mathrm{CH}_{3} \mathrm{CN} / \mathrm{H}_{2} \mathrm{O}(1: 2,2 \mathrm{~mL})$ and the solutions were lyophilized. 
4.8.1. (5R,3S)-1,4-Dibenzyl-5-((S)-1-(3-benzylureido)-2-(1H-indol-3-yl)ethyl-3-(3-(2((2,2,4,6,7-pentamethyl-2,3-dihydrobenzofuran-5-yl)sulfonyl)guanidino)propyl)-2oxopiperazine [(R)-28a]

Amorphous solid (46 mg, 50\%); $[\alpha]_{\mathrm{D}}{ }^{20}-13\left(c\right.$ 1.2, $\left.\mathrm{CH}_{2} \mathrm{Cl}_{2}\right)$; HPLC [Sunfire $\mathrm{C}_{18}(4.6 \times 150$ $\mathrm{mm}, 3.5 \mu \mathrm{m}), 50-100 \%$ gradient of solvent $\mathrm{A}$ in $\mathrm{B}, 30 \mathrm{~min}] t_{\mathrm{R}} 11.23 \mathrm{~min} ;{ }^{1} \mathrm{H}$ NMR $(400 \mathrm{MHz}$, $\left.\left(\mathrm{CD}_{3}\right)_{2} \mathrm{CO}\right) \delta(\mathrm{ppm}): 1.42$ [s, 6H, 2CH $\left.3(\mathrm{Pbf})\right], 1.79$ [m, 4H, $\beta-\mathrm{H}$ and $\left.\gamma-\mathrm{H}(\mathrm{Arg})\right], 2.02$ [s, 3H, $\left.\mathrm{CH}_{3}(\mathrm{Pbf})\right], 2.49$ [s, 3H, CH $\left.3(\mathrm{Pbf})\right], 2.58$ [s, 3H, $\left.\mathrm{CH}_{3}(\mathrm{Pbf})\right], 2.92$ [s, 2H, $\left.\mathrm{CH}_{2}(\mathrm{Pbf})\right], 2.98$ (m, $1 \mathrm{H}, \mathrm{CH}_{2}$-Ind), 3.06 [m, 2H, $\delta$-H (Arg)], $3.11[\mathrm{~m}, 1 \mathrm{H}, 3-\mathrm{H}], 3.39(\mathrm{dd}, 1 \mathrm{H}, J=4.5$ and $12.5 \mathrm{~Hz}$, 6-H), 3.49 [m, 1H, 4-CH $\left.\mathrm{CH}_{2}(\mathrm{Bn})\right], 3.53$ [m, 2H, $\mathrm{CH}_{2}$-Ind and 5-H], 3.65 (t, 1H, J = $12.5 \mathrm{~Hz}, 6-$ $\mathrm{H}), 4.20\left[\mathrm{~m}, 2 \mathrm{H}, \mathrm{CH}_{2}\right.$ (Bn, Urea)], $4.23\left[\mathrm{~m}, 1 \mathrm{H}, 4-\mathrm{CH}_{2}(\mathrm{Bn})\right], 4.40[\mathrm{~m}, 1 \mathrm{H}, 5-\mathrm{CH}], 4.51$ [d, $\left.1 \mathrm{H}, J=15 \mathrm{~Hz}, 1-\mathrm{CH}_{2}(\mathrm{Bn})\right], 4.69\left[\mathrm{~d}, 1 \mathrm{H}, J=15 \mathrm{~Hz}, 1-\mathrm{CH}_{2}(\mathrm{Bn})\right], 5.55$ (d, 1H, $J=9.5 \mathrm{~Hz}, 5-$ $\mathrm{CH}-\mathrm{NH}), 5.79(\mathrm{~m}, 1 \mathrm{H}, \mathrm{CONH}), 6.52$ [br s, 3H, NHC(NH$\left.)_{2}=\mathrm{N}\right], 6.91(\mathrm{t}, 1 \mathrm{H}, J=7.5 \mathrm{~Hz}, \mathrm{Ar})$, 7.05 (t, 1H, $J=7.5 \mathrm{~Hz}, \mathrm{Ar}), 7.11$ (d, 1H, J=7.5 Hz, Ar), 7.14-7.39 (m, 15H, Ar), 7.41 (d, 1H, $J=7.5 \mathrm{~Hz}, \mathrm{Ar}), 7.49$ (d, 1H, $J=7.5 \mathrm{~Hz}, \mathrm{Ar}), 10.08$ [s, 1H, NH (Ar)]; ${ }^{13} \mathrm{C} \mathrm{NMR}(100 \mathrm{MHz}$, $\left.\left(\mathrm{CD}_{3}\right)_{2} \mathrm{CO}\right) \delta(\mathrm{ppm}): 12.6,18.3,19.5\left[3 \mathrm{CH}_{3}(\mathrm{Pbf})\right], 27.2\left(\mathrm{CH}_{2}\right.$-Ind), 28.1 [C $\left.(\mathrm{Arg})\right], 28.7$ [2 $\left.\mathrm{CH}_{3}(\mathrm{Pbf})\right], 28.8\left[\mathrm{C}_{\beta}(\mathrm{Arg})\right], 41.2\left[\mathrm{C}_{\delta}(\mathrm{Arg})\right], 43.5\left[\mathrm{CH}_{2}(\mathrm{Pbf})\right], 44.1\left[\mathrm{CH}_{2}(\mathrm{Bn}, \mathrm{Urea})\right], 46.2$ $\left(\mathrm{C}_{6}\right), 50.4\left[1-\mathrm{CH}_{2}(\mathrm{Bn})\right], 51.4\left(\mathrm{C}_{5}-\mathrm{CH}\right), 51.9\left[4-\mathrm{CH}_{2}(\mathrm{Bn})\right], 55.5\left(\mathrm{C}_{5}\right), 62.2\left(\mathrm{C}_{3}\right), 87.0[\mathrm{C}$ (Pbf)], 112.1 [C (Ar)], 112.5 [CH (Ar)], 117.5 [C (Pbf)], 119.4, 121.9, 124.1 [4CH (Ar)], 125.3 [C (Pbf)], 127.3, 127.7, 128.1, 128.6, 129.3, 129.4, 129.9 [15CH (Ar)], 129.0 [C (Ar)], 132.8, 135.4 [2C (Pbf)], 137.5 [C (Ar)], 138.7 [C (Pbf)], 138.8, 139.6, 141.8 [C (Ar)], 157.4 $\left[\mathrm{C}\left(\mathrm{NHC}\left(\mathrm{NH}_{2}\right)=\mathrm{N}\right)\right], 158.8$ [C (Pbf)], 158.9 [CO (Urea)], $171.0\left(\mathrm{C}_{2}\right) ; \mathrm{ES}-\mathrm{MS} \mathrm{m} / \mathrm{z} 924.1$ $[\mathrm{M}+1]^{+}$; Anal. calcd. for $\mathrm{C}_{53} \mathrm{H}_{62} \mathrm{~N}_{8} \mathrm{O}_{5} \mathrm{~S}: \mathrm{C}, 68.95 ; \mathrm{H}, 6.77 ; \mathrm{N}, 12.14$. Found: C, 68.61; H, 6.89; $\mathrm{N}, 12.06$.

4.8.2. (5S,3S)-1,4-Dibenzyl-5-((S)-1-(3-benzylureido)-2-(1H-indol-3-yl)ethyl-3-(3-(2((2,2,4,6,7-pentamethyl-2,3-dihydrobenzofuran-5-yl)sulfonyl)guanidino)propyl)-2oxopiperazine [(S)-28a]

Amorphous solid (46 mg, 50\%); $[\alpha]_{\mathrm{D}}{ }^{20}+11\left(c\right.$ 1.1, $\left.\mathrm{CH}_{2} \mathrm{Cl}_{2}\right)$; HPLC [Sunfire $\mathrm{C}_{18}(4.6 \times 150$ $\mathrm{mm}, 3.5 \mu \mathrm{m}), 50-100 \%$ gradient of solvent $\mathrm{A}$ in $\mathrm{B}, 30 \mathrm{~min}] t_{\mathrm{R}} 13.75 \mathrm{~min} ;{ }^{1} \mathrm{H}$ NMR $(500 \mathrm{MHz}$, $\left.\left(\mathrm{CD}_{3}\right)_{2} \mathrm{CO}\right) \delta(\mathrm{ppm}): 1.30[\mathrm{~m}, 1 \mathrm{H}, \gamma-\mathrm{H}(\mathrm{Arg})], 1.43$ [s, 6H, 2CH $\left.3(\mathrm{Pbf})\right], 1.54[\mathrm{~m}, 2 \mathrm{H}, \beta-\mathrm{H}$ and $\gamma$-H (Arg)], 1.73 [m, 1H, $\beta-\mathrm{H}$ (Arg)], 2.04 [s, 3H, $\mathrm{CH}_{3}$ (Pbf)], 2.49 [s, 3H, $\mathrm{CH}_{3}$ (Pbf)], 2.57 [s, $\left.3 \mathrm{H}, \mathrm{CH}_{3}(\mathrm{Pbf})\right], 2.77$ (m, 1H, $\mathrm{CH}_{2}$-Ind), 2.88 [m, 1H, $\left.\delta-\mathrm{H}(\mathrm{Arg})\right], 2.96$ [s, 2H, $\mathrm{CH}_{2}$ (Pbf)], 
$3.09[\mathrm{~m}, 1 \mathrm{H}, \delta-\mathrm{H}(\mathrm{Arg})], 3.10$ [m, 1H, 3-H], $3.12\left(\mathrm{~m}, 2 \mathrm{H}, 5-\mathrm{H}\right.$ and $\left.\mathrm{CH}_{2}-\mathrm{Ind}\right), 3.47$ (m, 2H, 6H), $3.74\left[\mathrm{~d}, 1 \mathrm{H}, J=13.5 \mathrm{~Hz}, 4-\mathrm{CH}_{2}(\mathrm{Bn})\right], 3.96\left[\mathrm{~d}, 1 \mathrm{H}, J=13.5 \mathrm{~Hz}, 4-\mathrm{CH}_{2}(\mathrm{Bn})\right], 4.24[\mathrm{~m}$, $1 \mathrm{H}, 5-\mathrm{CH}], 4.26$ [dd, $1 \mathrm{H}, J=5.5$ and $15 \mathrm{~Hz}, \mathrm{CH}_{2}$ (Bn, Urea)], 4.30 [dd, $1 \mathrm{H}, J=6$ and $15 \mathrm{~Hz}$, $\mathrm{CH}_{2}$ (Bn, Urea)], 4.46 [d, $\left.1 \mathrm{H}, J=15 \mathrm{~Hz}, 1-\mathrm{CH}_{2}(\mathrm{Bn})\right], 4.71$ [d, $\left.1 \mathrm{H}, J=15 \mathrm{~Hz}, 1-\mathrm{CH}_{2}(\mathrm{Bn})\right]$, $5.70(\mathrm{~d}, 1 \mathrm{H}, J=8.5 \mathrm{~Hz}, 5-\mathrm{CH}-\mathrm{NH}), 6.03(\mathrm{~m}, 1 \mathrm{H}, \mathrm{CONH}), 6.45$ [br s, 3H, NHC( $\left.\mathrm{NH}_{2}\right)=\mathrm{N}$ ], 6.96 (t, 1H, $J=7.5 \mathrm{~Hz}, \mathrm{Ar}), 7.02$ (m, 1H, Ar), 7.08 (t, 1H, $J=7.5 \mathrm{~Hz}, \mathrm{Ar}), 7.15-7.39$ (m, 15H, Ar), $7.40(\mathrm{~d}, 1 \mathrm{H}, J=7.5 \mathrm{~Hz}, \mathrm{Ar}), 7.53(\mathrm{~d}, 1 \mathrm{H}, J=7.5 \mathrm{~Hz}, \mathrm{Ar}), 10.00[\mathrm{~s}, 1 \mathrm{H}, \mathrm{NH}(\mathrm{Ar})] ;{ }^{13} \mathrm{C}$

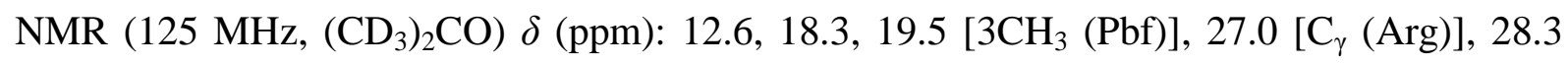
$\left(\mathrm{CH}_{2}\right.$-Ind), 28.7 [2 $\mathrm{CH}_{3}$ (Pbf)], $31.1\left[\mathrm{C}_{\beta}(\mathrm{Arg})\right], 40.9\left[\mathrm{C}_{\delta}(\mathrm{Arg})\right], 43.6\left[\mathrm{CH}_{2}(\mathrm{Pbf})\right], 44.3\left[\mathrm{CH}_{2}\right.$ (Bn, Urea)], $45.9\left(\mathrm{C}_{6}\right), 49.7$ [1- $\left.\mathrm{CH}_{2}(\mathrm{Bn})\right], 54.1\left(\mathrm{C}_{5}-\mathrm{CH}\right), 62.9\left[\mathrm{C}_{5}\right.$ and 4- $\left.\mathrm{CH}_{2}(\mathrm{Bn})\right], 63.6\left(\mathrm{C}_{3}\right)$, 86.9 [C (Pbf)], 112.1 [C and CH (Ar)], 117.4 [C (Pbf)], 119.4, 122.0, 123.7 [4CH (Ar)], 125.4 [C (Pbf)], 127.4, 127.9, 128.1, 128.2, 128.8, 129.5, 130.3 [15CH (Ar)], 129.1 [C (Ar)], 132.8, 135.7 [2C (Pbf)], 137.5 [C (Ar)], 138.7 [C (Pbf) and C (Ar)], 140.0, 141.7 [2C (Ar)], 157.5 [C $\left.\left(\mathrm{NHC}\left(\mathrm{NH}_{2}\right)=\mathrm{N}\right)\right], 158.9$ [C $\left.(\mathrm{Pbf})\right], 159.2$ [CO (Urea)], $172.1\left(\mathrm{C}_{2}\right)$; ES-MS m/z $924.2[\mathrm{M}+1]^{+}$; Anal. calcd. for $\mathrm{C}_{53} \mathrm{H}_{62} \mathrm{~N}_{8} \mathrm{O}_{5} \mathrm{~S}: \mathrm{C}: 68.95, \mathrm{H}: 6.77, \mathrm{~N}: 12.14$. Found: C: 68.59, H: 6.98, N: 12.02 .

4.8.3. (5R,3S)-1,4-Dibenzyl-5-((S)-1-(3-benzylureido)-2-phenylethyl)-3-(3-(2-((2,2,4,6,7pentamethyl-2,3-dihydrobenzofuran-5-yl)sulfonyl)guanidino) propyl)-2-oxopiperazine [(R)-29a]

Amorphous solid (62 mg, 70\%); $[\alpha]_{\mathrm{D}}{ }^{20}-14\left(c\right.$ 1.3, $\left.\mathrm{CH}_{2} \mathrm{Cl}_{2}\right)$; HPLC [Sunfire $\mathrm{C}_{18}(4.6 \times 150$ $\mathrm{mm}, 3.5 \mu \mathrm{m}), 60-100 \%$ gradient of solvent $\mathrm{A}$ in $\mathrm{B}, 30 \mathrm{~min}] t_{\mathrm{R}} 8.12 \mathrm{~min} ;{ }^{1} \mathrm{H}$ NMR $(400 \mathrm{MHz}$, $\left.\left(\mathrm{CD}_{3}\right)_{2} \mathrm{CO}\right) \delta$ (ppm): $1.43\left[\mathrm{~s}, 6 \mathrm{H}, 2 \mathrm{CH}_{3}(\mathrm{Pbf})\right], 1.79$ [m, 4H, $\beta-\mathrm{H}$ and $\left.\gamma-\mathrm{H}(\mathrm{Arg})\right], 2.03$ [s, 3H, $\mathrm{CH}_{3}$ (Pbf)], 2.49 [s, 3H, $\mathrm{CH}_{3}$ (Pbf)], 2.57 [s, 3H, $\mathrm{CH}_{3}$ (Pbf)], 2.76 (dd, 1H, J= 11 and $14 \mathrm{~Hz}$, $\left.\mathrm{CH}_{2} \mathrm{-Ph}\right), 2.96\left[\mathrm{~s}, 2 \mathrm{H}, \mathrm{CH}_{2}(\mathrm{Pbf})\right], 3.07$ [m, 2H, $\left.\delta-\mathrm{H}(\mathrm{Arg})\right], 3.12$ [m, 1H, 3-H], $3.38(\mathrm{dd}, 1 \mathrm{H}, J$ $=4.5$ and $12.5 \mathrm{~Hz}, 6-\mathrm{H}), 3.48\left[\mathrm{~m}, 1 \mathrm{H}, 4-\mathrm{CH}_{2}(\mathrm{Bn})\right], 3.49\left(\mathrm{~m}, 1 \mathrm{H}, \mathrm{CH}_{2}-\mathrm{Ph}\right), 3.50(\mathrm{~m}, 1 \mathrm{H}, 5-\mathrm{H})$, 3.63 (t, 1H, J = $12.5 \mathrm{~Hz}, 6-\mathrm{H}), 4.18$ [m, 2H, $\mathrm{CH}_{2}$ (Bn, Urea)], 4.20 [m, 1H, 4- $\mathrm{CH}_{2}$ (Bn)], 4.28 $(\mathrm{m}, 1 \mathrm{H}, 5-\mathrm{CH}), 4.53$ [d, 1H, $\left.J=15 \mathrm{~Hz}, 1-\mathrm{CH}_{2}(\mathrm{Bn})\right], 4.68\left[\mathrm{~d}, 1 \mathrm{H}, J=15 \mathrm{~Hz}, 1-\mathrm{CH}_{2}(\mathrm{Bn})\right]$, $5.59(\mathrm{~d}, 1 \mathrm{H}, J=9 \mathrm{~Hz}, 5-\mathrm{CH}-N H), 5.77(\mathrm{t}, 1 \mathrm{H}, J=6 \mathrm{~Hz}, \mathrm{CONH}), 6.50[\mathrm{br} \mathrm{s}, 3 \mathrm{H}$, $\left.\mathrm{NHC}\left(\mathrm{NH}_{2}\right)=\mathrm{N}\right], 7.04-7.43(\mathrm{~m}, 20 \mathrm{H}, \mathrm{Ar}) ;{ }^{13} \mathrm{C} \mathrm{NMR}\left(100 \mathrm{MHz},\left(\mathrm{CD}_{3}\right)_{2} \mathrm{CO}\right) \delta(\mathrm{ppm}): 12.6$, 18.2, $19.5\left[3 \mathrm{CH}_{3}(\mathrm{Pbf})\right], 27.1\left[\mathrm{C}_{\gamma}(\mathrm{Arg})\right], 28.7\left[\mathrm{C}_{\beta}(\mathrm{Arg})\right.$ and $\left.2 \mathrm{CH}_{3}(\mathrm{Pbf})\right], 38.6\left(\mathrm{CH}_{2}-\mathrm{Ph}\right), 41.3$ $\left[\mathrm{C}_{\delta}(\mathrm{Arg})\right], 43.6\left[\mathrm{CH}_{2}(\mathrm{Pbf})\right], 44.1\left[\mathrm{CH}_{2}(\mathrm{Bn}, \mathrm{Urea})\right], 46.2\left(\mathrm{C}_{6}\right), 50.4$ [1- $\left.\mathrm{CH}_{2}(\mathrm{Bn})\right], 51.8$ [4$\mathrm{CH}_{2}(\mathrm{Bn})$ ], $52.5\left(\mathrm{C}_{5^{-}} \mathrm{CH}\right), 55.8\left(\mathrm{C}_{5}\right), 62.1\left(\mathrm{C}_{3}\right), 86.9,117.5,125.3$ [3C (Pbf)], 126.8, 127.3, 
128.1, 128.7, 128.9, 129.0, 129.3, 129.4, 129.9, 130.3 [20CH (Ar)], 132.8, 135.5 [2C (Pbf)], 138.7 [C (Ar)], 138.8 [C (Pbf)], 139.6, 140.3, 141.8 [3C (Ar)], 157.4 [C $\left.\left(\mathrm{NHC}\left(\mathrm{NH}_{2}\right)=\mathrm{N}\right)\right]$, 158.6 [CO (Urea)], 158.9 [C (Pbf)], $171.0\left(\mathrm{C}_{2}\right)$; ES-MS m/z 885.0 [M+1] ${ }^{+}$; Anal. calcd. for $\mathrm{C}_{51} \mathrm{H}_{61} \mathrm{~N}_{7} \mathrm{O}_{5} \mathrm{~S}: \mathrm{C}, 69.28 ; \mathrm{H}, 6.95 ; \mathrm{N}, 11.09$. Found: C, 69.54; H, 7.12; N, 10.86.

4.8.4. (5S,3S)-1,4-Dibenzyl-5-((S)-1-(3-benzylureido)-2-phenylethyl)-3-(3-(2-((2,2,4,6,7pentamethyl-2,3-dihydrobenzofuran-5-yl)sulfonyl)guanidino) propyl)-2-oxopiperazine [(S)-29a]

Amorphous solid (75 mg, 85\%); $[\alpha]_{\mathrm{D}}{ }^{20}+9.9\left(c 1.2, \mathrm{CH}_{2} \mathrm{Cl}_{2}\right)$; HPLC [Sunfire $\mathrm{C}_{18}(4.6 \times 150$ $\mathrm{mm}, 3.5 \mu \mathrm{m}), 60-100 \%$ gradient of solvent $\mathrm{A}$ in $\mathrm{B}, 30 \mathrm{~min}] t_{\mathrm{R}} 11.52 \mathrm{~min} ;{ }^{1} \mathrm{H}$ NMR $(400 \mathrm{MHz}$, $\left.\left(\mathrm{CD}_{3}\right)_{2} \mathrm{CO}\right) \delta(\mathrm{ppm}): 1.43$ [s, 6H, 2CH $\left.3(\mathrm{Pbf})\right], 1.54$ [m, 2H, $\left.\gamma-\mathrm{H}(\mathrm{Arg})\right], 1.70[\mathrm{~m}, 2 \mathrm{H}, \beta-\mathrm{H}$ (Arg)], 2.04 [s, 3H, CH $(\mathrm{Pbf})$ ], 2.49 [s, 3H, CH $(\mathrm{Pbf})$ ], 2.56 (m, 1H, $\left.\mathrm{CH}_{2} \mathrm{Ph}\right), 2.57$ [s, 3H, $\mathrm{CH}_{3}$ (Pbf)], 2.98 (m, 1H, $\left.\mathrm{CH}_{2}-\mathrm{Ph}\right), 2.99$ [s, 2H, CH (Pbf)], 3.08 [m, 2H, $\delta$-H (Arg)], 3.10 [m, $1 \mathrm{H}, 3-\mathrm{H}$ and 5-H], 3.45 (d, 2H, $J=8 \mathrm{~Hz}, 6-\mathrm{H}), 3.75$ [d, 1H, $\left.J=13.5 \mathrm{~Hz}, 4-\mathrm{CH}_{2}(\mathrm{Bn})\right], 3.99$ [d, $\left.1 \mathrm{H}, J=13.5 \mathrm{~Hz}, 4-\mathrm{CH}_{2}(\mathrm{Bn})\right], 4.15(\mathrm{~m}, 1 \mathrm{H}, 5-\mathrm{CH}), 4.18$ [dd, $1 \mathrm{H}, \mathrm{J}=5.5$ and $15 \mathrm{~Hz}, \mathrm{CH}_{2}(\mathrm{Bn}$, Urea)], 4.27 [dd, $1 \mathrm{H}, \mathrm{J}=6.5$ and $15 \mathrm{~Hz}, \mathrm{CH}_{2}\left(\mathrm{Bn}\right.$, Urea)], 4.51 [d, $1 \mathrm{H}, J=15 \mathrm{~Hz}, 1-\mathrm{CH}_{2}$ $(\mathrm{Bn})], 4.68\left[\mathrm{~d}, 1 \mathrm{H}, J=15 \mathrm{~Hz}, 1-\mathrm{CH}_{2}(\mathrm{Bn})\right], 5.75$ (d, 1H, $\left.J=9 \mathrm{~Hz}, 5-\mathrm{CH}-\mathrm{NH}\right), 5.97(\mathrm{~m}, 1 \mathrm{H}$, $\mathrm{CONH}), 6.40\left[\mathrm{br} \mathrm{s}, 3 \mathrm{H}, \mathrm{NHC}\left(\mathrm{NH}_{2}\right)=\mathrm{N}\right], 7.07-7.45(\mathrm{~m}, 20 \mathrm{H}, \mathrm{Ar}) ;{ }^{13} \mathrm{C} \mathrm{NMR}(100 \mathrm{MHz}$, $\left.\left(\mathrm{CD}_{3}\right)_{2} \mathrm{CO}\right) \delta(\mathrm{ppm}): 12.6,18.3,19.5\left[3 \mathrm{CH}_{3}(\mathrm{Pbf})\right], 26.9\left[\mathrm{C}_{\gamma}(\mathrm{Arg})\right], 28.7\left[2 \mathrm{CH}_{3}(\mathrm{Pbf})\right]$, 31.4[C $\left.\mathrm{C}_{\beta}(\mathrm{Arg})\right], 38.4\left(\mathrm{CH}_{2}-\mathrm{Ph}\right), 41.0\left[\mathrm{C}_{\delta}(\mathrm{Arg})\right], 43.7\left[\mathrm{CH}_{2}(\mathrm{Pbf})\right], 44.2\left[\mathrm{CH}_{2}\right.$ (Bn, Urea)], 45.8 $\left(\mathrm{C}_{6}\right), 49.8\left[1-\mathrm{CH}_{2}(\mathrm{Bn})\right], 54.9\left(\mathrm{C}_{5}-\mathrm{CH}\right), 63.1\left[4-\mathrm{CH}_{2}(\mathrm{Bn})\right], 63.6\left(\mathrm{C}_{5}\right), 63.8\left(\mathrm{C}_{3}\right)$, 87.0, 117.5, 125.4 [3C (Pbf)], 126.9, 127.4, 127.8, 128.1, 128.2, 128.7, 129.0, 129.1, 129.2, 129.5, 130.1, 130.3 [20CH (Ar)], 132.8, 135.7 [2C (Pbf)], 138.6 [C (Ar)], 138.7 [C (Pbf)], 140.0, 141.6 [3C (Ar)], 157.5 [C (NHC( $\left.\left.\left.\mathrm{NH}_{2}\right)=\mathrm{N}\right)\right], 158.9$ [C (Pbf)], 159.9 [CO (Urea)], $172.1\left(\mathrm{C}_{2}\right) ; \mathrm{ES}-\mathrm{MS} \mathrm{m} / \mathrm{z}$ 885.0 [M+1] $]^{+}$; Anal. calcd. for $\mathrm{C}_{51} \mathrm{H}_{61} \mathrm{~N}_{7} \mathrm{O}_{5} \mathrm{~S}: \mathrm{C}, 69.28 ; \mathrm{H}, 6.95 ; \mathrm{N}, 11.09$. Found: C, 69.03; $\mathrm{H}, 7.21 ; \mathrm{N}, 10.97$.

4.9. General procedure for the synthesis of the Arg derived $\alpha$-amino nitriles (RS)-32a and $33 a$

TEA $(279 \mu \mathrm{L}, 2 \mathrm{mmol} \mathrm{mmol})$ was added to a solution of $\mathrm{H}-\mathrm{Arg}(\mathrm{Pbf})-\mathrm{OMe} \cdot \mathrm{HCl}(954 \mathrm{mg}, 2$ $\mathrm{mmol}$ ) in $\mathrm{MeOH}(25 \mathrm{~mL})$. After $15 \mathrm{~min}$ of stirring at rt, $\mathrm{ZnCl}_{2}$ (272 mg, $\left.2 \mathrm{mmol}\right)$ was added, followed by the addition of the corresponding aldehyde $\mathbf{3 0}(295 \mathrm{mg}, 2.2 \mathrm{mmol})$ or $\mathbf{3 1}[57,58]$ (601 mg, $2.2 \mathrm{mmol}$, freshly prepared as indicated in the Supplementary content) and the 
mixture was stirred for $1 \mathrm{~h}$. Then, TMSCN was added ( $375 \mu \mathrm{L}, 3 \mathrm{mmol})$ and the mixture was stirred overnight at $\mathrm{rt}$. The solvent was removed under reduced pressure and the residue was processed as above indicated in the general procedure for the synthesis of $\Psi[\mathrm{CH}(\mathrm{CN}) \mathrm{NH}]$ pseudodipeptides to give the $\alpha$-amino nitriles $(\boldsymbol{R S})-\mathbf{3 2 a}$ and $\mathbf{- 3 3 a}$.

\subsubsection{N-((RS)-1-Cyano-3-phenylpropyl)-Arg(Pbf)-OMe [(RS)-32a]}

White solid (992 mg, $85 \%)$ ); HPLC-MS [Sunfire $\mathrm{C}_{18}(4.6 \times 50 \mathrm{~mm}, 3.5 \mu \mathrm{m}), 10-100 \%$ gradient of solvent $\mathrm{A}$ in $\mathrm{B}, 5 \mathrm{~min}] t_{\mathrm{R}} 5.52 \mathrm{~min} ;{ }^{1} \mathrm{H} \mathrm{NMR}\left(300 \mathrm{MHz}, \mathrm{CDCl}_{3}\right) \delta(\mathrm{ppm}): 1.45[\mathrm{~s}$, $6 \mathrm{H}, 2 \mathrm{CH}_{3}$ (Pbf)], 1.49-1.85 [m, 4H, $\beta-\mathrm{H}$ and $\left.\gamma-\mathrm{H}(\mathrm{Arg})\right], 1.95-2.14$ (m, 2H, 3-H), 2.03 [s, 3H, $\mathrm{CH}_{3}$ (Pbf)], 2.50 [s, 3H, $\mathrm{CH}_{3}$ (Pbf)], 2.56 [s, 3H, $\mathrm{CH}_{3}$ (Pbf)], 2.68-2.89 (m, 2H, 4-H), 2.94 [s, $2 \mathrm{H}, \mathrm{CH}_{2}$ (Pbf)], 3.08-3.24 [m, 2H, $\delta$-H (Arg)], 3.32 and 3.43 [2t, 1H, J = 6 Hz, $\alpha-\mathrm{H}$ (Arg)], 3.46 and $3.55(2 \mathrm{t}, 1 \mathrm{H}, \mathrm{J}=6 \mathrm{~Hz}, 2-\mathrm{H}), 3.71$ and $3.74(2 \mathrm{~s}, 3 \mathrm{H}, \mathrm{OMe}), 5.81$ and 5.99 (br s, 3H, $\left.\mathrm{NHC}\left(\mathrm{NH}_{2}\right)=\mathrm{N}\right), 7.14-7.31(\mathrm{~m}, 5 \mathrm{H}, \mathrm{Ph}) ;{ }^{13} \mathrm{C} \mathrm{NMR}\left(75 \mathrm{MHz}, \mathrm{CDCl}_{3}\right) \delta(\mathrm{ppm}): 12.6,18.1,19.4$ [ $\left.3 \mathrm{CH}_{3}(\mathrm{Pbf})\right], 25.7$ and $25.9\left[\mathrm{C}_{\gamma}(\mathrm{Arg})\right], 28.7\left[2 \mathrm{CH}_{3}(\mathrm{Pbf})\right], 30.5$ and $30.8\left[\mathrm{C}_{\beta}(\mathrm{Arg})\right], 31.7$ $\left(\mathrm{C}_{4}\right), 35.5$ and $35.8\left(\mathrm{C}_{3}\right), 40.9$ and $43.3\left[\mathrm{CH}_{2}(\mathrm{Pbf})\right], 48.7$ and $48.9\left(\mathrm{C}_{2}\right), 52.6(\mathrm{OMe}), 58.8$ and $59.7\left[\mathrm{C}_{\alpha}(\mathrm{Arg})\right], 86.6,117.7,124.8$ [3C (Pbf)], $120.1(\mathrm{CN}), 126.6,128.5,128.8(5 \mathrm{CH}, \mathrm{Ph})$, 132.3, 132.9 (2C, Ph), 138.4, 139.9, 158.9 [3C (Pbf)], 156.3[C $\left.\left(\mathrm{NHC}\left(\mathrm{NH}_{2}\right)=\mathrm{N}\right)\right], 174.3,174.5$ $\left(\mathrm{CO}_{2}\right)$; ES-MS m/z 583.2828 [M+1] $]^{+}$; Anal. calcd. for $\mathrm{C}_{30} \mathrm{H}_{41} \mathrm{~N}_{5} \mathrm{O}_{5} \mathrm{~S}: \mathrm{C}, 61.73 ; \mathrm{H}, 7.08 ; \mathrm{N}$, 12.00. Found: C, 62.03; H, 7.21; N, 11.87 .

4.9.2. N-((RS)-3-(1-(tert-Butoxycarbonyl)-indol-3-yl)-1-cyanopropyl)-Arg(Pbf)-OMe [(RS)$33 a]$

White solid (751 mg, $52 \%)$ ); HPLC-MS [Sunfire $\mathrm{C}_{18}(4.6 \times 50 \mathrm{~mm}, 3.5 \mu \mathrm{m}), 10-100 \%$ gradient of solvent $\mathrm{A}$ in $\mathrm{B}, 5 \mathrm{~min}] t_{\mathrm{R}} 6.29 \mathrm{~min} ;{ }^{1} \mathrm{H} \mathrm{NMR}\left(300 \mathrm{MHz}, \mathrm{CDCl}_{3}\right) \delta(\mathrm{ppm}): 1.44$ [s, 6H, 2 $\mathrm{CH}_{3}$ (Pbf)], 1.67 (s. 9H, Boc), 1.53-1.89 [m, 4H, $\beta-\mathrm{H}$ and $\gamma-\mathrm{H}$ (Arg)], 2.08 [s, 3H, $\mathrm{CH}_{3}$ (Pbf)], 2.17 (m, 2H, 3-H), 2.51 [s, 3H, $\mathrm{CH}_{3}$ (Pbf)], 2.57 [s, 3H, $\mathrm{CH}_{3}$ (Pbf)], 2.80-3.05 (m, 2H, 4-H), $2.93\left[\mathrm{~s}, 2 \mathrm{H}, \mathrm{CH}_{2}(\mathrm{Pbf})\right], 3.07-3.27[\mathrm{~m}, 2 \mathrm{H}, \delta-\mathrm{H}(\mathrm{Arg})], 3.32$ and $3.48[\mathrm{~m}, 1 \mathrm{H}, \alpha-\mathrm{H}$ (Arg)], 3.47 and 3.64 (m, 1 H, 2-H), 3.69 and 3.74 (2s, 3H, OMe), 5.71 and 5.96 [br s, 3H, $\mathrm{NHC}\left(\mathrm{NH}_{2}\right)=\mathrm{N}$ ], 7.21-7.41 (m, 4H, Ind), 7.51 [d, $1 \mathrm{H}, \mathrm{J}=8 \mathrm{~Hz}, 4-\mathrm{H}$ (Ind)]; ${ }^{13} \mathrm{C} \mathrm{NMR}(75$ $\left.\mathrm{MHz}, \mathrm{CDCl}_{3}\right) \delta(\mathrm{ppm}): 12.6,18.1,19.4\left[3 \mathrm{CH}_{3}(\mathrm{Pbf})\right], 20.09$ and $21.1\left(\mathrm{C}_{4}\right), 25.3$ and $25.5\left[\mathrm{C}_{\gamma}\right.$ (Arg) $], 28.4\left[3 \mathrm{CH}_{3}(\mathrm{Boc})\right], 28.7\left[2 \mathrm{CH}_{3}(\mathrm{Pbf})\right], 30.0$ and $30.3\left[\mathrm{C}_{\beta}(\mathrm{Arg})\right], 33.1$ and $33.2\left(\mathrm{C}_{3}\right)$, $41.1\left[\mathrm{C}_{\delta}(\mathrm{Arg})\right], 43.3\left[\mathrm{CH}_{2}(\mathrm{Pbf})\right], 48.5$ and $48.9\left(\mathrm{C}_{2}\right), 52.4$ and $52.5(\mathrm{OMe}), 58.7$ and $59.4\left[\mathrm{C}_{\alpha}\right.$ 
(Arg)], 83.9 (C, Boc), 86.6, 115.5, 123.1 [3C (Pbf)], $115.5\left(\mathrm{C}_{3}\right.$, Ind), 118.9 and $119.1(\mathrm{CN})$, 118.6, 118.7, 119.0, 119.1, 119.9, 120.2, 122.7, 122.9, 124.8, 124.9 (CH, Ind), 130.2, 130.3 $135.6\left(\mathrm{C}_{3 \mathrm{a}}\right.$ and $\mathrm{C}_{7 \mathrm{a}}$, Ind $), 132.6,138.7,155.9$ [3C $\left.(\mathrm{Pbf})\right], 159.0\left[\mathrm{C}\left(\mathrm{NHC}\left(\mathrm{NH}_{2}\right)=\mathrm{N}\right)\right], 174.2$ $\left(\mathrm{CO}_{2}\right)$; ES-MS m/z $722.3476[\mathrm{M}+1]^{+}$; Anal. calcd. for $\mathrm{C}_{37} \mathrm{H}_{50} \mathrm{~N}_{6} \mathrm{O}_{7} \mathrm{~S}: \mathrm{C}, 61.47 ; \mathrm{H}, 6.97 ; \mathrm{N}$, 11.63. Found: C, 61.63; H, 7.11; N, 11.47 .

4.10. General procedure for the synthesis of the 2-oxopiperazine derivatives (R)- and (S)$34 a$ and $-35 a$

Raney-Ni (2.4 g, previously washed with $\mathrm{MeOH})$ and hydrazine monohydrate $(1.26 \mathrm{~mL}$, $26 \mathrm{mmol}$ ) were added to a solution of the corresponding epimeric mixture of $\alpha$-amino nitrile $(\boldsymbol{R S})$-32a and -33a $(2.06 \mathrm{mmol})$ in $\mathrm{MeOH}(50 \mathrm{~mL})$ and the mixture was heated at $65^{\circ} \mathrm{C}$ for 20 min. Afterward, the reaction mixture was filtered over celite and the solvent was evaporated under reduced pressure. The residue was purified by flash chromatography, by using $\mathrm{MeOH}$ in $\mathrm{CH}_{2} \mathrm{Cl}_{2}$ gradient as mobile phase to obtain the desired resolved 2-oxopiperazines $(\boldsymbol{R})$ - and (S)-34a and -35a.

\subsection{1. (5R,3S)-3-(3-(2-((2,2,4,6,7-Pentamethyl-2,3-dihydrobenzofuran-5-yl)sulfonyl)- guanidino)propyl)-5-phenethyl-2-oxopiperazine [(R)-34a]}

White solid (345 mg, $31 \%)$ ); $[\alpha]_{\mathrm{D}}{ }^{20}-0.4$ (c 2, $\left.\mathrm{CHCl}_{3}\right)$; Mp: 129-131 ${ }^{\circ} \mathrm{C}$; HPLC-MS [Sunfire $\mathrm{C}_{18}(4.6 \times 50 \mathrm{~mm}, 3.5 \mu \mathrm{m}), 10-100 \%$ gradient of solvent $\mathrm{A}$ in $\mathrm{B}, 5 \mathrm{~min}$ ] $t_{\mathrm{R}} 3.80 \mathrm{~min}$; ${ }^{1} \mathrm{H}$ NMR (400 MHz, $\left.\mathrm{CDCl}_{3}\right) \delta(\mathrm{ppm}): 1.37$ [s, 6H, 2CH $\left.3(\mathrm{Pbf})\right], 1.53-1.91[\mathrm{~m}, 6 \mathrm{H}, 1-\mathrm{H}$ (phenethyl), $\beta-\mathrm{H}$ and $\gamma-\mathrm{H}$ (Arg)], 2.00 [s, 3H, $\mathrm{CH}_{3}$ (Pbf)], 2.09 (br s, 1H, 4-H), 2.43 [s, 3H, $\mathrm{CH}_{3}$ (Pbf)], 2.49 [s, 3H, $\mathrm{CH}_{3}$ (Pbf)], 2.57 and 2.64 [2m, 2H, 2-H (phenethyl)], 2.85 [s, 2H, $\mathrm{CH}_{2}$ (Pbf)], $2.88(\mathrm{~m}, 1 \mathrm{H}, 5-\mathrm{H}), 2.99-3.24$ [m, 4H, 6- $\mathrm{H}$ and $\left.\delta-\mathrm{H}(\mathrm{Arg})\right], 3.31(\mathrm{~m}, 1 \mathrm{H}, 3-\mathrm{H})$, 6.32 (br s, 1H, 1-H), 6.43 and 6.89 [br s, 3H, $\left.\mathrm{NHC}\left(\mathrm{NH}_{2}\right)=\mathrm{N}\right]$, 7.07-7.12 and 7.17-7.20 (2 m, $5 \mathrm{H}, \mathrm{Ph}) ;{ }^{13} \mathrm{C} \mathrm{NMR}\left(100 \mathrm{MHz}, \mathrm{CDCl}_{3}\right) \delta(\mathrm{ppm}): 12.6,18.1,19.4\left[3 \mathrm{CH}_{3}(\mathrm{Pbf})\right], 26.1\left[\mathrm{C}_{\gamma}\right.$ (Arg)], 28.7 [2 $\mathrm{CH}_{3}$ (Pbf)], $29.5\left[\mathrm{C}_{\beta}\right.$ (Arg)], 32.3 [C 2 (phenethyl)], 35.0 [ $\mathrm{C}_{1}$ (phenethyl)], 41.0 $\left[\mathrm{C}_{\delta}(\mathrm{Arg})\right], 43.4\left[\mathrm{CH}_{2}(\mathrm{Pbf})\right], 46.6\left(\mathrm{C}_{5}\right), 48.1\left(\mathrm{C}_{6}\right), 53.3\left(\mathrm{C}_{3}\right), 86.5,117.5,124.7$ [3C $\left.(\mathrm{Pbf})\right]$, 126.2, 128.4, 128.6 (5 CH, Ph), 141.4 (C, Ph), 132.4, 133.2 , 138.4, 158.7 [4C (Pbf)], 156.7 $\left[\mathrm{C}\left(\mathrm{NHC}\left(\mathrm{NH}_{2}\right)=\mathrm{N}\right)\right], 173.3\left(\mathrm{C}_{2}\right)$; ES-MS $m / z$ 556.2950 [M+1] $]^{+}$; Anal. calcd. for $\mathrm{C}_{29} \mathrm{H}_{41} \mathrm{~N}_{5} \mathrm{O}_{4} \mathrm{~S}$ : C, 62.68; H, 7.44; N, 12.60. Found: C, 62.83; H, 7.51; N, 12.47. 


\subsection{2. (5S,3S)-3-(3-(2-((2,2,4,6,7-Pentamethyl-2,3-dihydrobenzofuran-5-yl)sulfonyl)-}

\section{guanidino)propyl)-5-phenethyl-2-oxopiperazine [(S)-34a]}

White solid (389 mg, $35 \%)$ ); $[\alpha]_{\mathrm{D}}{ }^{20}-0.4\left(c\right.$ 2, $\left.\mathrm{CHCl}_{3}\right)$; Mp: 128-130 ${ }^{\circ} \mathrm{C}$; HPLC-MS [Sunfire $\mathrm{C}_{18}(4.6 \times 50 \mathrm{~mm}, 3.5 \mu \mathrm{m}), 10-100 \%$ gradient of solvent $\mathrm{A}$ in $\mathrm{B}, 5 \mathrm{~min}$ ] $t_{\mathrm{R}} 3.83 \mathrm{~min}$; ${ }^{1} \mathrm{H}$ NMR (400 MHz, $\left.\mathrm{CDCl}_{3}\right) \delta(\mathrm{ppm}): 1.44\left[\mathrm{~s}, 6 \mathrm{H}, 2 \mathrm{CH}_{3}(\mathrm{Pbf})\right], 1.57-1.92[\mathrm{~m}, 6 \mathrm{H}, 1-\mathrm{H}$ (phenethyl), $\beta-\mathrm{H}$ and $\gamma-\mathrm{H}$ (Arg)], 2.07 [s, 3H, $\mathrm{CH}_{3}$ (Pbf)], 2.49 [s, 3H, $\mathrm{CH}_{3}(\mathrm{Pbf})$ ], 2.56 [s, 3H, $\mathrm{CH}_{3}$ (Pbf)], 2.67 [2m, 2H, 2-H (phenethyl)], 2.93 [s, 2H, $\mathrm{CH}_{2}$ (Pbf)], 3.00 (m, 1H, 5-H), 3.18 $[\mathrm{m}, 4 \mathrm{H}, 6-\mathrm{H}$ and $\delta-\mathrm{H}(\mathrm{Arg})], 3.50(\mathrm{~m}, 1 \mathrm{H}, 3-\mathrm{H}), 6.28-6.56\left[\mathrm{br} \mathrm{s}, 3 \mathrm{H}, \mathrm{NHC}\left(\mathrm{NH}_{2}\right)=\mathrm{N}\right], 6.86(\mathrm{br}$ s, 1H, 1-H), 7.14-7.20 and 7.24-7.28 (2 m, 5H, Ph); $\left.{ }^{13} \mathrm{C} \mathrm{NMR} \mathrm{(100} \mathrm{MHz}, \mathrm{CDCl}_{3}\right) \delta$ (ppm): 12.6, 18.1, $19.5\left[3 \mathrm{CH}_{3}(\mathrm{Pbf})\right], 25.4\left[\mathrm{C}_{\gamma}(\mathrm{Arg})\right], 28.7\left[2 \mathrm{CH}_{3}(\mathrm{Pbf})\right], 29.9\left[\mathrm{C}_{\beta}(\mathrm{Arg})\right], 32.3\left[\mathrm{C}_{2}\right.$ (phenethyl)], $35.0\left[\mathrm{C}_{1}\right.$ (phenethyl)], $41.1\left[\mathrm{C}_{\delta}(\mathrm{Arg})\right], 43.4\left[\mathrm{CH}_{2}(\mathrm{Pbf})\right], 48.0\left(\mathrm{C}_{6}\right), 52.2\left(\mathrm{C}_{5}\right)$, 58.3 $\left(\mathrm{C}_{3}\right), 86.5,117.6,124.7$ [3C (Pbf)], 126.3, 128.4, 128.8 (5 CH, Ph), $141.2(\mathrm{C}, \mathrm{Ph}), 132.4$, 133.2, 138.4, 158.8 [4C (Pbf)], $156.5\left[\mathrm{C}\left(\mathrm{NHC}\left(\mathrm{NH}_{2}\right)=\mathrm{N}\right)\right], 172.5\left(\mathrm{C}_{2}\right)$; ES-MS m/z 556.2953 $[\mathrm{M}+1]^{+}$; Anal. calcd. for $\mathrm{C}_{29} \mathrm{H}_{41} \mathrm{~N}_{5} \mathrm{O}_{4} \mathrm{~S}: \mathrm{C}, 62.68 ; \mathrm{H}, 7.44 ; \mathrm{N}, 12.60$. Found: C, 62.81; H, 7.53; $\mathrm{N}, 12.55$.

4.10.3. (5R,3S)-5-(2-(1-(tert-Butoxycarbonyl)-indol-3-yl)ethyl-3-(3-(2-((2,2,4,6,7pentamethyl-2,3-dihydrobenzofuran-5-yl)sulfonyl)guanidino)propyl)-2-oxopiperazine [(R)-35a]

White solid (361 mg, $26 \%)$ ); $[\alpha]_{\mathrm{D}}{ }^{20}-5.8$ (c 2, $\left.\mathrm{CHCl}_{3}\right)$; Mp: 124-126 ${ }^{\circ} \mathrm{C}$; HPLC-MS [Sunfire $\mathrm{C}_{18}(4.6 \times 50 \mathrm{~mm}, 3.5 \mu \mathrm{m}), 10-100 \%$ gradient of solvent $\mathrm{A}$ in $\mathrm{B}, 5 \mathrm{~min}$ ] $t_{\mathrm{R}} 4.10 \mathrm{~min}$; ${ }^{1} \mathrm{H}$ NMR (400 MHz, $\mathrm{CDCl}_{3}$ ) $\delta(\mathrm{ppm}): 1.36$ [s, 6H, 2CH 3 (Pbf)], 1.58 (s, 9H, Boc), 1.50-1.90 [m, 6H, 1-H (phenethyl), $\beta-\mathrm{H}$ and $\gamma-\mathrm{H}(\mathrm{Arg})], 1.99\left[\mathrm{~s}, 3 \mathrm{H}, \mathrm{CH}_{3}(\mathrm{Pbf})\right], 2.42\left[\mathrm{~s}, 3 \mathrm{H}, \mathrm{CH}_{3}\right.$ (Pbf)], 2.49 [s, 3H, $\mathrm{CH}_{3}$ (Pbf)], 2.62-2.80 [2m, 2H, 2-H (phenethyl)], 2.83 [s, 2H, $\mathrm{CH}_{2}$ (Pbf)], $2.97(\mathrm{~m}, 1 \mathrm{H}, 5-\mathrm{H}), 3.02-3.28[\mathrm{~m}, 4 \mathrm{H}, 6-\mathrm{H}$ and $\delta-\mathrm{H}(\mathrm{Arg})], 3.37(\mathrm{~m}, 1 \mathrm{H}, 3-\mathrm{H}), 6.34$ (br s, 1H, 1-H), 6.45 [br s, 3H, NHC( $\left.\left.\mathrm{NH}_{2}\right)=\mathrm{N}\right], 7.14$ [t, 1H, J = $7 \mathrm{~Hz}, 5-\mathrm{H}($ Ind)], 7.23 [t, 1H, J = $7 \mathrm{~Hz}$, 6-H (Ind)], 7.28 [s, 1H, 2-H, (Ind)], 7.42 [d, 1H, J = 7 Hz, 7-H, (Ind)], 8.02 [ br s, 1H, 4-H, (Ind)]; ${ }^{13} \mathrm{C}$ NMR (100 MHz, $\left.\mathrm{CDCl}_{3}\right) \delta(\mathrm{ppm}): 12.6,18.1,19.4\left[3 \mathrm{CH}_{3}(\mathrm{Pbf})\right], 21.5\left[\mathrm{C}_{2}\right.$ (phenethyl)], $26.1\left[\mathrm{C}_{\gamma}(\mathrm{Arg})\right], 28.4\left[3 \mathrm{CH}_{3}(\mathrm{Boc})\right], 28.7\left[2 \mathrm{CH}_{3}(\mathrm{Pbf})\right], 29.5\left[\mathrm{C}_{\beta}(\mathrm{Arg})\right], 32.3\left[\mathrm{C}_{1}\right.$ (phenethyl)], $40.9\left[\mathrm{C}_{\delta}(\mathrm{Arg})\right], 43.3\left[\mathrm{CH}_{2}(\mathrm{Pbf})\right], 46.6\left(\mathrm{C}_{5}\right), 48.0\left(\mathrm{C}_{6}\right), 55.4\left(\mathrm{C}_{3}\right), 83.8(\mathrm{C}, \mathrm{Boc})$, 86.5, 117.6, 120.2 [3C (Pbf)], 115.4, 119.1, 122.5, 124,6 (5 CH, Ind), 130.5, 133.2 (2C, Ind), 132.4, 138.5, 158.8 [3C (Pbf)], $150.0(\mathrm{CO}, \mathrm{Boc}), 156.7$ [C $\left.\left(\mathrm{NHC}\left(\mathrm{NH}_{2}\right)=\mathrm{N}\right)\right], 173.3\left(\mathrm{C}_{2}\right)$; ES- 
MS m/z 695.3601 [M+1] $]^{+}$; Anal. calcd. for $\mathrm{C}_{36} \mathrm{H}_{50} \mathrm{~N}_{6} \mathrm{O}_{6} \mathrm{~S}: \mathrm{C}, 62.22 ; \mathrm{H}, 7.25 ; \mathrm{N}, 12.09$. Found: C, 62.41; H, 7.33; N, 11.92 .

4.10.4. (5S,3S)-5-(2-(1-(tert-Butoxycarbonyl)-indol-3-yl)ethyl-3-(3-(2-((2,2,4,6,7-

pentamethyl-2,3-dihydrobenzofuran-5-yl)sulfonyl)guanidino)propyl)-2-oxopiperazine [(S)-35a]

White solid (278 mg, $20 \%)$ ); $[\alpha]_{\mathrm{D}}{ }^{20}-4.4\left(c\right.$ 2, $\left.\mathrm{CHCl}_{3}\right)$; Mp: 134-136 ${ }^{\circ} \mathrm{C}$; HPLC-MS [Sunfire $\mathrm{C}_{18}(4.6 \times 50 \mathrm{~mm}, 3.5 \mu \mathrm{m}), 10-100 \%$ gradient of solvent $\mathrm{A}$ in $\mathrm{B}, 5 \mathrm{~min}$ ] $t_{\mathrm{R}} 4.12 \mathrm{~min}$; ${ }^{1} \mathrm{H}$ NMR (400 MHz, $\mathrm{CDCl}_{3}$ ) $\delta$ (ppm): 1.36 [s, 6H, 2CH (Pbf)], 1.59 (s, 9H, Boc), 1.57-1.86 [m, 6H, 1-H (phenethyl), $\beta-\mathrm{H}$ and $\gamma-\mathrm{H}(\mathrm{Arg})], 2.00\left[\mathrm{~s}, 3 \mathrm{H}, \mathrm{CH}_{3}(\mathrm{Pbf})\right], 2.42\left[\mathrm{~s}, 3 \mathrm{H}, \mathrm{CH}_{3}\right.$ (Pbf)], 2.50 [s, 3H, $\mathrm{CH}_{3}$ (Pbf)], 2.70 [m, 2H, 2-H (phenethyl)], 2.84 [s, 2H, $\mathrm{CH}_{2}$ (Pbf)], 2.91$3.22[\mathrm{~m}, 5 \mathrm{H}, 5-\mathrm{H}, 6-\mathrm{H}$ and $\delta-\mathrm{H}(\mathrm{Arg})], 3.41(\mathrm{~m}, 1 \mathrm{H}, 3-\mathrm{H}), 6.26$ [br s, 3H, $\left.\mathrm{NHC}\left(\mathrm{NH}_{2}\right)=\mathrm{N}\right]$, 6.67 (br s, 1H, 1-H), 7.14 [t, 1H, J = 7 Hz, 5-H (Ind)], 7.23 [t, 1H, J = 7 Hz, 6-H (Ind)], 7.31 [s, 1H, 2-H, (Ind)], 7.43 [d, 1H, J = $7 \mathrm{~Hz}, 7-\mathrm{H}$, (Ind)], 8.03 [ br s, 1H, 4-H, (Ind)]; ${ }^{13} \mathrm{C} \mathrm{NMR}$ $\left(100 \mathrm{MHz}, \mathrm{CDCl}_{3}\right) \delta(\mathrm{ppm}): 12.6,18.1,19.4\left[3 \mathrm{CH}_{3}(\mathrm{Pbf})\right], 21.4\left[\mathrm{C}_{2}\right.$ (phenethyl)], $25.4\left[\mathrm{C}_{\gamma}\right.$

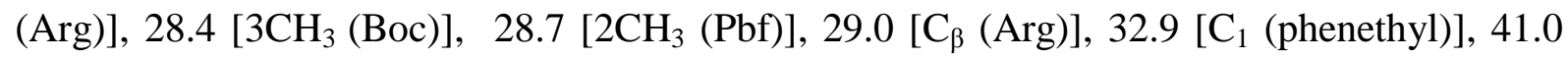
[C $\left.\mathrm{C}_{\delta}(\mathrm{Arg})\right], 43.3\left[\mathrm{CH}_{2}(\mathrm{Pbf})\right], 48.0\left(\mathrm{C}_{6}\right), 52.3\left(\mathrm{C}_{5}\right), 58.3\left(\mathrm{C}_{3}\right), 83.8(\mathrm{C}, \mathrm{Boc}), 86.5,117.6,120.1$ [3C (Pbf)], 115.5, 119.0, 122.6, 124,6 (5 CH, Ind), 130.5, 133.1 (2C, Ind), 132.4, 138.5, 158.8 [3C (Pbf)], $150.0(\mathrm{CO}, \mathrm{Boc}), 156.5$ [C $\left.\left(\mathrm{NHC}\left(\mathrm{NH}_{2}\right)=\mathrm{N}\right)\right], 172.3\left(\mathrm{C}_{2}\right)$; ES-MS m/z 695.3593 $[\mathrm{M}+1]^{+}$; Anal. calcd. for $\mathrm{C}_{36} \mathrm{H}_{50} \mathrm{~N}_{6} \mathrm{O}_{6} \mathrm{~S}: \mathrm{C}, 62.22 ; \mathrm{H}, 7.25 ; \mathrm{N}, 12.09$. Found: C, 62.45; H, 7.27; $\mathrm{N}, 11.87$.

4.11. General procedure for the removal of the Pbf protecting group in (R)- and (S)-34a. Synthesis of (R)- and (S)-36a

The corresponding protected 2-oxopiperazine derivative $(\boldsymbol{R})$ - and $(\boldsymbol{S})$-34a $(93 \mathrm{mg}, 0.17$ mmol) was dissolved in (95:2.5:2.5) TFA: $\mathrm{H}_{2} \mathrm{O}$ :TIPS mixture $(1 \mathrm{~mL})$. After $24 \mathrm{~h}$ of stirring at $\mathrm{rt}$, the solvents were evaporated to dryness and the residue was lyophilized to give quantitatively the respective deprotected derivative $(\boldsymbol{R})$ - and $(\boldsymbol{S})$-36a.

\subsection{1.(5R,3S)-3-(3-Guanidino)propyl)-5-phenethyl-2-oxopiperazine [(R)-36a]}

trifluoroacetate

Amorphous solid (89 mg, $100 \%)$ ); $[\alpha]_{\mathrm{D}}^{20}-2.3$ (c 1, DMSO); HPLC-MS [Sunfire $\mathrm{C}_{18}$ (4.6×50 mm, $3.5 \mu \mathrm{m}), 2-30 \%$ gradient of solvent $\mathrm{A}$ in $\mathrm{B}, 5 \mathrm{~min}] t_{\mathrm{R}} 1.02 \mathrm{~min} ;{ }^{1} \mathrm{H} \mathrm{NMR}(400$ 
$\left.\mathrm{MHz}, \mathrm{D}_{2} \mathrm{O}\right) \delta(\mathrm{ppm}):$ 1.54-2.08 [m, 6H, 1-H (phenethyl), $\beta-\mathrm{H}$ (Arg) and $\left.\gamma-\mathrm{H}(\mathrm{Arg})\right], 2.59$ [m, 2H, 2-H (phenethyl)], 3.05 [t, 2H, J = $6 \mathrm{~Hz}, \delta-\mathrm{H}(\mathrm{Arg})], 3.28$ (t, 1H, J = $12 \mathrm{~Hz}, 6-\mathrm{H}) 3.42$ (m, 1H, 5-H), 3.49 (dd, 1H, J = 4 and $12 \mathrm{~Hz}, 6-\mathrm{H}), 3.84$ (t, 1H, J = $6 \mathrm{~Hz}, 3-\mathrm{H}), 7.09-7.22$ (m, 5H, $\mathrm{Ph}) ;{ }^{13} \mathrm{C}$ NMR (100 MHz, D $\left.\mathrm{D}_{2} \mathrm{O}\right) \delta(\mathrm{ppm}): 24.4\left[\mathrm{C}_{\gamma}(\mathrm{Arg})\right], 26.3\left[\mathrm{C}_{\beta}(\mathrm{Arg})\right], 30.4\left[\mathrm{C}_{2}\right.$ (phenethyl)], $31.3\left[\mathrm{C}_{1}\right.$ (phenethyl)], $40.5\left[\mathrm{C}_{\delta}(\mathrm{Arg})\right], 42.3\left(\mathrm{C}_{6}\right), 52.5\left(\mathrm{C}_{5}\right), 56.3\left(\mathrm{C}_{3}\right), 126.8$,

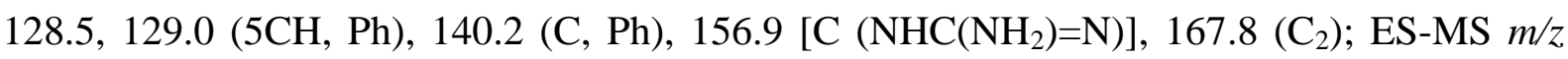
$304.43[\mathrm{M}+1]^{+}$.

\subsection{2. (5S,3S)-3-(3-Guanidino)propyl)-5-phenethyl-2-oxopiperazine [(S)-36a]}

Amorphous solid (90 mg, $100 \%)$ ); $[\alpha]_{\mathrm{D}}^{20}-5.4$ (c 1, DMSO); HPLC-MS [Sunfire $\mathrm{C}_{18}$ (4.6×50 mm, $3.5 \mu \mathrm{m}), 2-30 \%$ gradient of solvent $\mathrm{A}$ in $\mathrm{B}, 5 \mathrm{~min}] t_{\mathrm{R}} 1.01 \mathrm{~min} ;{ }^{1} \mathrm{H}$ NMR (400 $\left.\mathrm{MHz}, \mathrm{D}_{2} \mathrm{O}\right) \delta(\mathrm{ppm}): 1.53$ [m, 2H, $\left.\gamma-\mathrm{H}(\mathrm{Arg})\right], 1.68-1.9$ [m, 2H, $\left.\beta-\mathrm{H}(\mathrm{Arg})\right], 1.80-2.01$ [m, 2H, 1-H (phenethyl)], 2.58 [m, 2H, 2-H (phenethyl)], 2.98 [m, 2H, $\delta$-H (Arg)], 3.25 (dd, 1H, J = 9 and $15 \mathrm{~Hz}, 6-\mathrm{H}) 3.41-3.51(\mathrm{~m}, 2 \mathrm{H}, 5-\mathrm{H}$ and 6-H), 3.85 (t, 1H, J = $7 \mathrm{~Hz}, 3-\mathrm{H}), 7.06-7.19$ (m, $5 \mathrm{H}, \mathrm{Ph}) ;{ }^{13} \mathrm{C}$ NMR (100 MHz, $\left.\mathrm{D}_{2} \mathrm{O}\right) \delta(\mathrm{ppm}): 24.7\left[\mathrm{C}_{\gamma}(\mathrm{Arg})\right], 26.8\left[\mathrm{C}_{\beta}(\mathrm{Arg})\right], 30.1\left[\mathrm{C}_{2}\right.$ (phenethyl)], 30.6 [ $\mathrm{C}_{1}$ (phenethyl)], 40.4 [ $\mathrm{C}_{\delta}$ (Arg)], $41.7\left(\mathrm{C}_{6}\right), 49.1\left(\mathrm{C}_{5}\right), 53.9\left(\mathrm{C}_{3}\right), 126.8$,

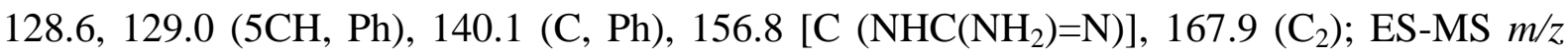
$304.36[\mathrm{M}+1]^{+}$.

4.12. General procedure for the $N_{4}$-benzylation of the 2-oxopiperazines $34 a$ and $35 a$. Synthesis of the 4-benzyl-2-oxopiperazines (R)- and (S)-(37a and $\mathbf{3 8 a})$

DIEA $(190 \mu \mathrm{L}, 1.1 \mathrm{mmol})$ and benzyl bromide $(260 \mu \mathrm{L}, 2.2 \mathrm{mmol})$ were added under argon a solution of the corresponding 2-oxopiperazine $(\boldsymbol{R})$ - and $(\boldsymbol{S})-\mathbf{6 a}, \mathbf{b}$ and $\mathbf{- 7 \mathbf { a }}(0.55 \mathrm{mmol})$ in anhydrous $\mathrm{CH}_{3} \mathrm{CN}(5 \mathrm{~mL})$ at $60{ }^{\circ} \mathrm{C}$. After $3 \mathrm{~h}$ of stirring, the solvent was removed under reduced pressure. The residue was processed as indicated for the benzylation of analogues $\mathbf{6 a}$ and $7 \mathbf{a}$.

4.12.1. (5R,3S)-4-Benzyl-3-(3-(2-((2,2,4,6,7-pentamethyl-2,3-dihydrobenzofuran-5yl)sulfonyl)-guanidino)propyl)-5-phenethyl-2-oxopiperazine [(R)-37a]

White solid (259 mg, $73 \%)$ ); $[\alpha]_{\mathrm{D}}{ }^{20}+0\left(c\right.$ 2, $\left.\mathrm{CHCl}_{3}\right)$; Mp: 89-91 ${ }^{\circ} \mathrm{C}$; HPLC-MS [Sunfire $\mathrm{C}_{18}(4.6 \times 50 \mathrm{~mm}, 3.5 \mu \mathrm{m}), 10-100 \%$ gradient of solvent $\mathrm{A}$ in $\left.\mathrm{B}, 5 \mathrm{~min}\right] t_{\mathrm{R}} 5.51 \mathrm{~min} ;{ }^{1} \mathrm{H}$ NMR $\left(400 \mathrm{MHz}, \mathrm{CDCl}_{3}\right) \delta(\mathrm{ppm}): 1.32[\mathrm{~m}, 1 \mathrm{H}, \gamma-\mathrm{H}(\mathrm{Arg})], 1.37$ [s, 6H, 2CH $\left.3(\mathrm{Pbf})\right], 1.43-1.74$ [m, 
4H, 1-H (phenethyl), $\beta-\mathrm{H}$ and $\gamma-\mathrm{H}$ (Arg)], 1.85 [m, 1H, 1- $\mathrm{H}$ (phenethyl)], 2.00 [s, 3H, $\mathrm{CH}_{3}$ (Pbf)], 2.42 [s, 3H, CH $(\mathrm{Pbf})], 2.49$ [s, 3H, $\left.\mathrm{CH}_{3}(\mathrm{Pbf})\right], 2.63$ and 2.72 [2m, 2H, 2-H (phenethyl)], 2.82 [m, 2H, $\delta-\mathrm{H}(\mathrm{Arg})], 2.85$ [s, 2H, $\mathrm{CH}_{2}$ (Pbf)], 2.97 (m, 1H, 3-H), 3.11 (m, $2 \mathrm{H}, 5-\mathrm{H}$ and 6-H), $3.23(\mathrm{t}, 1 \mathrm{H}, \mathrm{J}=12 \mathrm{~Hz}, 6-\mathrm{H}), 3.30$ and $3.84\left(2 \mathrm{~d}, 2 \mathrm{H}, \mathrm{J}=13 \mathrm{~Hz}, 4-\mathrm{CH}_{2}\right)$, 6.22 and 6.27 [2br s, $4 \mathrm{H}, 1-\mathrm{H}$ and $\left.\mathrm{NHC}\left(\mathrm{NH}_{2}\right)=\mathrm{N}\right], 7.09-7.23(\mathrm{~m}, 10 \mathrm{H}, \mathrm{Ph}) ;{ }^{13} \mathrm{C} \mathrm{NMR}(100$ $\left.\mathrm{MHz}, \mathrm{CDCl}_{3}\right) \delta(\mathrm{ppm}): 12.7,18.0,19.4\left[3 \mathrm{CH}_{3}(\mathrm{Pbf})\right], 25.7\left[\mathrm{C}_{\gamma}(\mathrm{Arg})\right], 28.8\left[\mathrm{C}_{\beta}(\mathrm{Arg})\right], 28.7$ [2 $\left.\mathrm{CH}_{3}(\mathrm{Pbf})\right], 30.3\left[\mathrm{C}_{1}\right.$ (phenethyl)], 32.4 [C $\mathrm{C}_{2}$ (phenethyl)], $40.7\left[\mathrm{C}_{\delta}(\mathrm{Arg})\right], 43.4\left[\mathrm{CH}_{2}(\mathrm{Pbf})\right]$, $43.1\left(\mathrm{C}_{6}\right), 49.7\left(\mathrm{C}_{5}\right), 50.8\left(4-\mathrm{CH}_{2}\right), 60.3\left(\mathrm{C}_{3}\right), 86.4,117.5,124.7$ [3C (Pbf)], 126.3, 128.5, $128.6(10 \mathrm{CH}, \mathrm{Ph}), 141.4(2 \mathrm{C}, \mathrm{Ph}), 132.4,133.2,138.5,158.7$ [4C (Pbf)], 156.5 [C $\left.\left(\mathrm{NHC}\left(\mathrm{NH}_{2}\right)=\mathrm{N}\right)\right], 173.3\left(\mathrm{C}_{2}\right)$; ES-MS $m / z 646.72[\mathrm{M}+1]^{+}$; Anal. calcd. for $\mathrm{C}_{36} \mathrm{H}_{47} \mathrm{~N}_{5} \mathrm{O}_{4} \mathrm{~S}: \mathrm{C}$, 66.95; H, 7.33; N, 10.84. Found: C, 67.06; H, 7.51; N, 10.70.

\subsection{2. (5S,3S)-4-Benzyl-3-(3-(2-((2,2,4,6,7-pentamethyl-2,3-dihydrobenzofuran-5- \\ yl)sulfonyl)-guanidino)propyl)-5-phenethyl-2-oxopiperazine [(S)-37a]}

White solid (255 mg, $72 \%)) ;[\alpha]_{\mathrm{D}}{ }^{20}+0 \quad\left(c 2, \mathrm{CHCl}_{3}\right)$; Mp: 73-75 ${ }^{\circ} \mathrm{C}$; HPLC-MS [Sunfire $\mathrm{C}_{18}(4.6 \times 50 \mathrm{~mm}, 3.5 \mu \mathrm{m}), 10-100 \%$ gradient of solvent $\mathrm{A}$ in $\left.\mathrm{B}, 5 \mathrm{~min}\right] t_{\mathrm{R}} 5.54 \mathrm{~min} ;{ }^{1} \mathrm{H}$ NMR $\left(400 \mathrm{MHz}, \mathrm{CDCl}_{3}\right) \delta(\mathrm{ppm}): 1.44$ [s, 6H, 2CH $\left.3(\mathrm{Pbf})\right], 1.54$ [m, 1H, $\left.\gamma-\mathrm{H}(\mathrm{Arg})\right], 1.70$ [m, 3H, 1-H (phenethyl) and $\beta-\mathrm{H}], 1.92$ [m, 1H, 1-H (phenethyl)], 2.08 [s, 3H, $\left.\mathrm{CH}_{3}(\mathrm{Pbf})\right], 2.49$ [s, $3 \mathrm{H}, \mathrm{CH}_{3}$ (Pbf)], 2.55 [s, 3H, $\mathrm{CH}_{3}$ (Pbf)], 2.55 and 2.65 [2m, 2H, 2-H (phenethyl)], 2.80 (m, 1H, 5-H), $2.82[\mathrm{~m}, 2 \mathrm{H}, \delta-\mathrm{H}(\mathrm{Arg})], 2.93\left[\mathrm{~s}, 2 \mathrm{H}, \mathrm{CH}_{2}(\mathrm{Pbf})\right], 3.03-3.23$ [m, 4H, 3-H, 6-H and $\delta$-H (Arg)], $3.40(\mathrm{~m}, 1 \mathrm{H}, 6-\mathrm{H}), 3.62$ and $3.81\left(2 \mathrm{~d}, 2 \mathrm{H}, \mathrm{J}=13 \mathrm{~Hz}, 4-\mathrm{CH}_{2}\right), 6.59$ [2br s, 4H, 1-H and $\left.\mathrm{NHC}\left(\mathrm{NH}_{2}\right)=\mathrm{N}\right], 7.09-7.30(\mathrm{~m}, 10 \mathrm{H}, \mathrm{Ph}) ;{ }^{13} \mathrm{C} \mathrm{NMR}\left(100 \mathrm{MHz}, \mathrm{CDCl}_{3}\right) \delta(\mathrm{ppm}): 12.7$, 18.0, $19.4\left[3 \mathrm{CH}_{3}(\mathrm{Pbf})\right], 25.7\left[\mathrm{C}_{\gamma}(\mathrm{Arg})\right], 28.7\left[2 \mathrm{CH}_{3}(\mathrm{Pbf})\right], 30.6\left[\mathrm{C}_{\beta}(\mathrm{Arg})\right], 32.1\left[\mathrm{C}_{2}\right.$ (phenethyl)], $35.2\left[\mathrm{C}_{1}\right.$ (phenethyl)], $40.7\left[\mathrm{C}_{\delta}(\mathrm{Arg})\right], 42.7\left(\mathrm{C}_{6}\right), 43.4\left[\mathrm{CH}_{2}(\mathrm{Pbf})\right], 57.6\left(\mathrm{C}_{5}\right)$, 59.4 (4- $\left.\mathrm{CH}_{2}\right), 62.1\left(\mathrm{C}_{3}\right), 86.5,117.6,124.7$ [3C (Pbf)], 126.3, 128.4, 128.6, 129.1 (10CH, Ph),

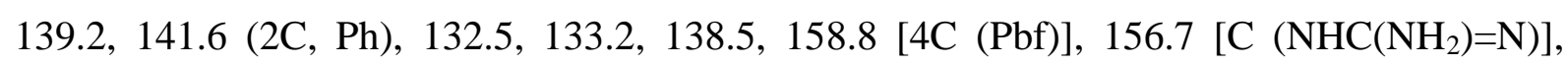
$174.1\left(\mathrm{C}_{2}\right)$; ES-MS $m / z 646.66[\mathrm{M}+1]^{+}$; Anal. calcd. for $\mathrm{C}_{36} \mathrm{H}_{47} \mathrm{~N}_{5} \mathrm{O}_{4} \mathrm{~S}: \mathrm{C}, 66.95 ; \mathrm{H}, 7.33 ; \mathrm{N}$, 10.84. Found: C, 66.86; H, 7.41; N, 10.68 .

4.12.3. (5R,3S)-4-Benzyl-5-(2-(1-(tert-butoxycarbonyl)-indol-3-yl)ethyl-3-(3-(2-((2,2,4,6,7pentamethyl-2,3-dihydrobenzofuran-5-yl)sulfonyl)guanidino)propyl)-2-oxopiperazine [(R)-38a] 
White solid (341 mg, $79 \%)$ ); $[\alpha]_{\mathrm{D}}{ }^{20}-1.4$ (c 2, $\mathrm{CHCl}_{3}$ ); Mp: 105-107 ${ }^{\circ} \mathrm{C}$; HPLC-MS [Sunfire $\mathrm{C}_{18}(4.6 \times 50 \mathrm{~mm}, 3.5 \mu \mathrm{m}), 10-100 \%$ gradient of solvent $\mathrm{A}$ in B, $5 \mathrm{~min}$ ] $t_{\mathrm{R}} 6.19 \mathrm{~min}$; ${ }^{1} \mathrm{H}$ NMR (400 MHz, $\mathrm{CDCl}_{3}$ ) $\delta$ (ppm): 1.43 [s, 6H, 2CH (Pbf)], 1.66 (s, 9H, Boc), 1.60-1.90 [m, 6H, 1-H (phenethyl), $\beta-\mathrm{H}$ and $\gamma-\mathrm{H}(\mathrm{Arg})], 2.07$ [s, 3H, $\left.\mathrm{CH}_{3}(\mathrm{Pbf})\right], 2.49$ [s, 3H, $\mathrm{CH}_{3}$ (Pbf)], 2.55 [s, 3H, $\mathrm{CH}_{3}$ (Pbf)], 2.82 [2m, 2H, 2-H (phenethyl)], 2.91 [s, 2H, $\mathrm{CH}_{2}$ (Pbf)], 2.96 [m, 2H, $\delta$-H (Arg)],3.11 (m, 1H, 3-H), 3.18-3.48 (m, 3H, 6-H and 4- $\left.\mathrm{CH}_{2}\right), 3.69(\mathrm{~m}, 1 \mathrm{H}, 5-\mathrm{H})$, $3.92\left(\mathrm{~d}, 1 \mathrm{H}, \mathrm{J}=13 \mathrm{~Hz}, 4-\mathrm{CH}_{2}\right), 6.33$ [br s, 3H, $\left.\mathrm{NHC}\left(\mathrm{NH}_{2}\right)=\mathrm{N}\right], 7.20-7.35(\mathrm{~m}, 9 \mathrm{H}, 1-\mathrm{H}$ and Ar), 7.49 [d, 1H, J = $8 \mathrm{~Hz}, 7-\mathrm{H}$, (Ind)], 8.09 [ d, 1H, J = $8 \mathrm{~Hz}, 4-\mathrm{H}$, (Ind)]; ${ }^{13} \mathrm{C} \mathrm{NMR}$ (100 $\left.\mathrm{MHz}, \mathrm{CDCl}_{3}\right) \delta(\mathrm{ppm}):$ 12.6, 18.0, 19.4 [3 $\left.\mathrm{CH}_{3}(\mathrm{Pbf})\right], 21.7\left[\mathrm{C}_{2}\right.$ (phenethyl)], $25.7\left[\mathrm{C}_{\gamma}(\mathrm{Arg})\right]$, $28.4\left[3 \mathrm{CH}_{3}(\mathrm{Boc})\right], 28.7$ [2 $\left.\mathrm{CH}_{3}(\mathrm{Pbf})\right], 29.4\left[\mathrm{C}_{\beta}(\mathrm{Arg})\right], 31.9$ [ $\mathrm{C}_{1}$ (phenethyl)], $40.7\left[\mathrm{C}_{\delta}\right.$ (Arg)], $43.0\left(\mathrm{C}_{6}\right), 43.3\left[\mathrm{CH}_{2}(\mathrm{Pbf})\right], 50.9\left(4-\mathrm{CH}_{2}\right), 54.2\left(\mathrm{C}_{5}\right), 60.6\left(\mathrm{C}_{3}\right), 83.8(\mathrm{C}, \mathrm{Boc}), 86.4,117.5$, 120.1 [3C (Pbf)], 115.5, 119.0, 122.6, 124,5, 124.6, 127.4, 128.5, 128.8, (10 CH, Ar), 130.4, 133.3 (2C, Ind), 135.6 (C, Ph), 132.3, 138.3, 158.6 [3C (Pbf)], 149.9 (CO, Boc), 156.5 [C $\left.\left(\mathrm{NHC}\left(\mathrm{NH}_{2}\right)=\mathrm{N}\right)\right], 173.3\left(\mathrm{C}_{2}\right)$; ES-MS $m / z 786.03[\mathrm{M}+1]^{+}$; Anal. calcd. for $\mathrm{C}_{43} \mathrm{H}_{56} \mathrm{~N}_{6} \mathrm{O}_{6} \mathrm{~S}: \mathrm{C}$, 65.79; H, 7.19; N, 10.71. Found: C, 65.92; H, 7.23; N, 10.82.

4.12.4. (5S,3S)-4-Benzyl-5-(2-(1-(tert-butoxycarbonyl)-indol-3-yl)ethyl-3-(3-(2-((2,2,4,6,7pentamethyl-2,3-dihydrobenzofuran-5-yl)sulfonyl)guanidino)propyl)-2-oxopiperazine [(S)-38a]

White solid (302 mg, $70 \%)$; $[\alpha]_{\mathrm{D}}{ }^{20}+1.2\left(c 2, \mathrm{CHCl}_{3}\right)$; Mp: 94-96 ${ }^{\circ} \mathrm{C}$; HPLC-MS [Sunfire $\mathrm{C}_{18}(4.6 \times 50 \mathrm{~mm}, 3.5 \mu \mathrm{m}), 10-100 \%$ gradient of solvent $\mathrm{A}$ in $\left.\mathrm{B}, 5 \mathrm{~min}\right] t_{\mathrm{R}} 6.15 \mathrm{~min} ;{ }^{1} \mathrm{H} \mathrm{NMR}$ (400 MHz, $\left.\mathrm{CDCl}_{3}\right) \delta$ (ppm): 1.36 [s, 6H, 2CH $\left.(\mathrm{Pbf})\right], 1.58$ (s, 9H, Boc), 1.40-1.77 [m, 5H, 1$\mathrm{H}$ (phenethyl), $\beta-\mathrm{H}$ and $\gamma-\mathrm{H}$ (Arg)], 1.92 [m, 1H, 1-H (phenethyl)], 2.00 [s, 3H, $\mathrm{CH}_{3}$ (Pbf)], 2.41 [s, 3H, $\mathrm{CH}_{3}$ (Pbf)], 2.48 [s, 3H, $\mathrm{CH}_{3}$ (Pbf)], 2.63 [m, 2H, 2-H (phenethyl)], 2.83 [s, 2H, $\mathrm{CH}_{2}$ (Pbf)], 2.96-3.20 [m, 4H, 3-H, 6-H, $\delta-\mathrm{H}$ (Arg)], 3.37 (m, 1H, 6-H), 3.59 (m, 2H, 5-H and 4- $\mathrm{CH}_{2}$ ), 3.77 (br s, 1H, 4- $\mathrm{CH}_{2}$ ), 6.26-6.91 [br s, 4H, 1-H, $\left.\mathrm{NHC}\left(\mathrm{NH}_{2}\right)=\mathrm{N}\right], 7.11-7.24$ (m, $8 \mathrm{H}, \mathrm{Ar}$ ), 7.35 [d, 1H, J = $8 \mathrm{~Hz}, 7-\mathrm{H}$, (Ind)], 8.02 [br s, 1H, J = $8 \mathrm{~Hz}, 4-\mathrm{H}$, (Ind)]; ${ }^{13} \mathrm{C} \mathrm{NMR}$ $\left(100 \mathrm{MHz}, \mathrm{CDCl}_{3}\right) \delta(\mathrm{ppm}): 12.6,17.9,19.4\left[3 \mathrm{CH}_{3}(\mathrm{Pbf})\right], 21.2\left[\mathrm{C}_{2}\right.$ (phenethyl)], $25.6\left[\mathrm{C}_{\gamma}\right.$ (Arg) $, 28.3\left[2 \mathrm{CH}_{3}(\mathrm{Pbf})\right], 28.6\left[3 \mathrm{CH}_{3}(\mathrm{Boc})\right], 30.2\left[\mathrm{C}_{\beta}(\mathrm{Arg})\right], 32.8\left[\mathrm{C}_{1}\right.$ (phenethyl)], $40.6\left[\mathrm{C}_{\delta}\right.$ (Arg) ], $42.6\left(\mathrm{C}_{6}\right), 43.2\left[\mathrm{CH}_{2}(\mathrm{Pbf})\right], 53.9\left(\mathrm{C}_{5}\right), 59.3\left(4-\mathrm{CH}_{2}\right), 62.1\left(\mathrm{C}_{3}\right), 83.5(\mathrm{C}, \mathrm{Boc}), 86.4$, 117.4, 120.3 [3C (Pbf)], 115.3, 118.9, 122.5, 124,4, 124.6, 127.4, 128.4, 128.8, (10 CH, Ar), 130.4, 133.2 (2C, Ind), 135.5 (C, Ph), 132.3, 138.4, 158.6 [3C (Pbf)], 149.8 (CO, Boc), 156.7 
$\left[\mathrm{C}\left(\mathrm{NHC}\left(\mathrm{NH}_{2}\right)=\mathrm{N}\right)\right], 174.1\left(\mathrm{C}_{2}\right)$; ES-MS m/z $785.95[\mathrm{M}+1]^{+}$; Anal. calcd. for $\mathrm{C}_{36} \mathrm{H}_{50} \mathrm{~N}_{6} \mathrm{O}_{6} \mathrm{~S}$ : C, 65.79; H, 7.19; N, 10.71. Found: C, 65.94; H, 7.13; N, 10.65.

\subsection{General procedure for the benzylation of the 4-benzyl-2-oxopiperazines 37a and 38a.} Synthesis of the 4-benzyl-2-oxopiperazines (R)- and (S)-(39a, 41a, 42a and 44a)

$\mathrm{NaH}(60 \%$ suspension in mineral oil, $9 \mathrm{mg}, 0.21 \mathrm{mmol})$ and benzyl bromide $(69 \mu \mathrm{L}, 0.23$ mmol) were added to a solution of the corresponding 2-oxopiperazine 37a and 38a $(0.21$ mmol) in anhydrous mixture THF/DMF $(9: 1,5 \mathrm{~mL})$ under argon at $0{ }^{\circ} \mathrm{C}$. After $24 \mathrm{~h}$ of stirring, the crude reaction mixture was diluted with EtOAc $(10 \mathrm{~mL})$ and the excess of $\mathrm{NaH}$ was hydrolysed by addition of $\mathrm{H}_{2} \mathrm{O}(2 \mathrm{~mL})$. The aqueous layer was extracted with EtOAc $(3 \times 5$ $\mathrm{mL}$ ) and the organic extracts were dried over $\mathrm{Na}_{2} \mathrm{SO}_{4}$ and evaporated to dryness. The residue was purified by flash chromatography, using 30-100 EtOAc gradient in hexane as mobile phase to give the respective 1,4-dibenzyl-2-oxopiperazines 39a and 42a, as solids, in 20-42\% yield and the respective derivative benzylated at the guanidino group 41a and 44a as $(Z / E)$ isomeric mixtures in 8-14 \% yield. These tribenzylated derivatives were dissolved in $\mathrm{CH}_{3} \mathrm{CN} / \mathrm{H}_{2} \mathrm{O}(1: 2,2 \mathrm{~mL})$ and the solutions were lyophilized to obtain amorphous solids.

\subsection{1. (5R,3S)-1,4-Dibenzyl-3-(3-(2-((2,2,4,6,7-pentamethyl-2,3-dihydrobenzofuran-5-yl)- sulfonyl)-guanidino)propyl)-5-phenethyl-2-oxopiperazine [(R)-39a]}

White solid (65 mg, $42 \%)$ ); $[\alpha]_{\mathrm{D}}{ }^{20}-3.1\left(c\right.$ 1, $\left.\mathrm{CHCl}_{3}\right)$; Mp: 93-95 ${ }^{\circ} \mathrm{C}$; HPLC-MS [Sunfire $\mathrm{C}_{18}(4.6 \times 50 \mathrm{~mm}, 3.5 \mu \mathrm{m}), 10-100 \%$ gradient of solvent $\mathrm{A}$ in $\left.\mathrm{B}, 5 \mathrm{~min}\right] t_{\mathrm{R}} 6.36 \mathrm{~min} ;{ }^{1} \mathrm{H}$ NMR (400 MHz, $\left.\mathrm{CDCl}_{3}\right) \delta(\mathrm{ppm}): 1.42$ [s, 6H, 2CH$\left.(\mathrm{Pbf})\right], 1.55$ [m, 2H, $\left.\gamma-\mathrm{H}(\mathrm{Arg})\right], 1.64$ and 1.86 [2m, 2H, 1-H (phenethyl)], 1.71 [m, 2H, $\beta-\mathrm{H}$ (Arg)], 2.05 [s, 3H, $\left.\mathrm{CH}_{3}(\mathrm{Pbf})\right], 2.48$ and 2.54 [2s, 6H, $\left.\mathrm{CH}_{3}(\mathrm{Pbf})\right], 2.54$ and 2.62 [2m, 2H, 2-H (phenethyl)], 2.89 [m, 4H, $\delta-\mathrm{H}(\mathrm{Arg})$ and $\mathrm{CH}_{2}$ (Pbf)], 3.03-3.24 (m, 4H, 3-H, 5-H, 6-H), 3.34 and 3.83 (2d, 2H, J =12 Hz, 4-CH $), 4.42$ and $4.62\left(2 \mathrm{~d}, 2 \mathrm{H}, \mathrm{J}=14 \mathrm{~Hz}, 1-\mathrm{CH}_{2}\right), 6.97$ and 6.08 [2br s, 3H, $\left.\mathrm{NHC}\left(\mathrm{NH}_{2}\right)=\mathrm{N}\right], 7.02-7.34(\mathrm{~m}$, $15 \mathrm{H}, \mathrm{Ph}) ;{ }^{13} \mathrm{C} \mathrm{NMR}\left(100 \mathrm{MHz}, \mathrm{CDCl}_{3}\right) \delta(\mathrm{ppm}): 12.6,18.0,19.4\left[3 \mathrm{CH}_{3}(\mathrm{Pbf})\right], 25.7\left[\mathrm{C}_{\gamma}\right.$

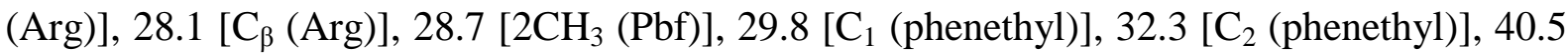
$\left[\mathrm{C}_{\delta}(\mathrm{Arg})\right], 43.3\left[\mathrm{CH}_{2}(\mathrm{Pbf})\right], 47.4\left(\mathrm{C}_{6}\right), 50.2\left(\mathrm{C}_{5}\right.$ and 1- $\left.\mathrm{CH}_{2}\right), 51.3\left(4-\mathrm{CH}_{2}\right), 61.0\left(\mathrm{C}_{3}\right), 86.4$, 117.5, 124.6 [3C (Pbf)], 126.2 and 127.9-129.2 (15CH, Ph), 136.6 and 141.4 (3C, Ph), 132.4, 133.2, 138.5, 158.7 [4C (Pbf)], 156.2 [C $\left(\mathrm{NHC}\left(\mathrm{NH}_{2}\right)=\mathrm{N}\right)$ ], $171.0\left(\mathrm{C}_{2}\right)$; ES-MS m/z 736.70 $[\mathrm{M}+1]^{+}$; Anal. calcd. for $\mathrm{C}_{43} \mathrm{H}_{53} \mathrm{~N}_{5} \mathrm{O}_{4} \mathrm{~S}: \mathrm{C}, 70.17 ; \mathrm{H}, 7.26 ; \mathrm{N}, 9.52$. Found: C, 70.29; H, 7.31; N, 9.36 . 
4.13.2. (5S,3S)-1,4-Dibenzyl-3-(3-(2-((2,2,4,6,7-pentamethyl-2,3-dihydrobenzofuran-5-yl)sulfonyl)-guanidino)propyl)-5-phenethyl-2-oxopiperazine [(S)-39a]

White solid (60 mg, $40 \%)$ ) $[\alpha]_{\mathrm{D}}{ }^{20}+1.7\left(c 1, \mathrm{CHCl}_{3}\right)$; Mp: 70-72 ${ }^{\circ} \mathrm{C}$; HPLC-MS [Sunfire $\mathrm{C}_{18}(4.6 \times 50 \mathrm{~mm}, 3.5 \mu \mathrm{m}), 10-100 \%$ gradient of solvent $\mathrm{A}$ in $\left.\mathrm{B}, 5 \mathrm{~min}\right] t_{\mathrm{R}} 6.20 \mathrm{~min} ;{ }^{1} \mathrm{H} \mathrm{NMR}$ $\left(400 \mathrm{MHz}, \mathrm{CDCl}_{3}\right) \delta(\mathrm{ppm}): 1.36$ [s, 6H, 2CH 3 (Pbf)], 1.39-1.65 [m, 5H, $\beta-\mathrm{H}, \gamma-\mathrm{H}$ (Arg), and 1-H (phenethyl)], 1.75 [m, 1H, 1-H (phenethyl)], 1.98 [s, 3H, $\mathrm{CH}_{3}$ (Pbf)], 2.34 [2m, 2H, 2-H (phenethyl)], 2.43 and 2.49 [2s, 6H, $\left.\mathrm{CH}_{3}(\mathrm{Pbf})\right], 2.67(\mathrm{~m}, 1 \mathrm{H}, 5-\mathrm{H}), 2.83$ [s, 2H, $\mathrm{CH}_{2}$ (Pbf)], $2.95[\mathrm{~m}, 2 \mathrm{H}, \delta-\mathrm{H}(\mathrm{Arg})], 3.05(\mathrm{dd}, 1 \mathrm{H}, \mathrm{J}=8$ and $13 \mathrm{~Hz}, 6-\mathrm{H}), 3.22$ (m, 2H, 3-H, 6-H), 3.57 and $3.65\left(2 \mathrm{~d}, 2 \mathrm{H}, \mathrm{J}=14 \mathrm{~Hz}, 4-\mathrm{CH}_{2}\right), 4.38$ and $4.52\left(2 \mathrm{~d}, 2 \mathrm{H}, \mathrm{J}=14.5 \mathrm{~Hz}, 1-\mathrm{CH}_{2}\right), 6.00$ and 6.09 [2br s, 3H, NHC( $\left.\left.\mathrm{NH}_{2}\right)=\mathrm{N}\right], 6.83(\mathrm{~d}, 2 \mathrm{H}, \mathrm{J}=7 \mathrm{~Hz}, \mathrm{Ph}), 7.06-7.24(\mathrm{~m}, 13 \mathrm{H}, \mathrm{Ph}) ;{ }^{13} \mathrm{C} \mathrm{NMR}$ $\left(100 \mathrm{MHz}, \mathrm{CDCl}_{3}\right) \delta(\mathrm{ppm}): 12.6,18.0,19.4\left[3 \mathrm{CH}_{3}(\mathrm{Pbf})\right], 25.6\left[\mathrm{C}_{\gamma}(\mathrm{Arg})\right], 28.7\left[2 \mathrm{CH}_{3}\right.$ (Pbf)], $29.8\left[\mathrm{C}_{\beta}\right.$ (Arg)], $30.6\left[\mathrm{C}_{1}\right.$ (phenethyl)], $32.1\left[\mathrm{C}_{2}\right.$ (phenethyl)], $40.8\left[\mathrm{C}_{\delta}\right.$ (Arg)], 43.3 $\left[\mathrm{CH}_{2}(\mathrm{Pbf})\right], 47.4\left(\mathrm{C}_{6}\right), 50.1\left(1-\mathrm{CH}_{2}\right), 56.7\left(\mathrm{C}_{5}\right), 58.7\left(4-\mathrm{CH}_{2}\right), 61.5\left(\mathrm{C}_{3}\right), 86.4,117.5,124.6$ [3C (Pbf)], 126.1 and 127.9-129.0 (15CH, Ph), 136.5 and 141.2 (3C, Ph), 132.4, 133.3, 138.4, $158.6[4 \mathrm{C}(\mathrm{Pbf})], 156.2\left[\mathrm{C}\left(\mathrm{NHC}\left(\mathrm{NH}_{2}\right)=\mathrm{N}\right)\right], 171.2\left(\mathrm{C}_{2}\right)$; ES-MS $m / z 736.74[\mathrm{M}+1]^{+}$; Anal. calcd. for $\mathrm{C}_{43} \mathrm{H}_{53} \mathrm{~N}_{5} \mathrm{O}_{4} \mathrm{~S}$ : C, 70.17; H, 7.26; N, 9.52. Found: C, 70.09; H, 7.27; N, 9.43.

\subsection{3. (5R,3S)-1,4-Dibenzyl-3-(3-(3-benzyl-2-((2,2,4,6,7-pentamethyl-2,3-dihydro-}

benzofuran-5-yl)sulfonyl)-guanidino)propyl)-5-phenethyl-2-oxopiperazine [(R)-41a]

Amorphous solid (22 mg, $13 \%)$ ); HPLC-MS [Sunfire $\mathrm{C}_{18}(4.6 \times 50 \mathrm{~mm}, 3.5 \mu \mathrm{m}), 10$ $100 \%$ gradient of solvent $\mathrm{A}$ in $\mathrm{B}, 5 \mathrm{~min}] t_{\mathrm{R}} 5.66(83 \%)$ and $5.85(17 \%) \mathrm{min} ;{ }^{1} \mathrm{H}$ NMR [500 $\left.\mathrm{MHz},\left(\mathrm{CD}_{3}\right)_{2} \mathrm{CO}\right] \delta$ (ppm): 1.41 and 1.42 [2s, 6H, 2 $\left.\mathrm{CH}_{3}(\mathrm{Pbf})\right], 1.57-1.78$ [m, $4 \mathrm{H}, \beta-\mathrm{H}$ and $\gamma-$ $\mathrm{H}(\mathrm{Arg})], 1.71$ and 1.94 [2m, 2H 1-H (phenethyl)], 2.01 [s, 3H, $\mathrm{CH}_{3}$ (Pbf)], 2.46 [s, 3H, $\mathrm{CH}_{3}$ (Pbf)], 2.52 [s, 3H, $\mathrm{CH}_{3}$ (Pbf)], 2.60 and 2.75 [2m, 2H, 2-H (phenethyl)], 2.93 [s, 2H, $\mathrm{CH}_{2}$ (Pbf)], 3.07 [m, 3H, 3-H and $\delta-\mathrm{H}(\mathrm{Arg})], 3.19(\mathrm{bd}, 1 \mathrm{H}, \mathrm{J}=10 \mathrm{~Hz}, 6-\mathrm{H}), 3.26(\mathrm{~m}, 1 \mathrm{H}, 3-\mathrm{H})$, $3.36(\mathrm{~m}, 1 \mathrm{H}, 6-\mathrm{H}), 3.39$ (d, 1H, J = $\left.14 \mathrm{~Hz}, 4-\mathrm{CH}_{2}\right), 3.94\left(\mathrm{~m}, 1 \mathrm{H}, 4-\mathrm{CH}_{2}\right), 4.43$ [d, 2H, J = 5 $\left.\mathrm{Hz}, \mathrm{NHC}\left(\mathrm{NH}-\mathrm{CH}_{2}\right)=\mathrm{N}\right], 4.53$ and $4.63\left(2 \mathrm{~d}, 2 \mathrm{H}, \mathrm{J}=14.5 \mathrm{~Hz}, 1-\mathrm{CH}_{2}\right), 7.10-7.37(\mathrm{~m}, 2 \mathrm{H}, \mathrm{Ph})$; ${ }^{13} \mathrm{C}$ NMR [125 MHz, $\left.\left(\mathrm{CD}_{3}\right)_{2} \mathrm{CO}\right] \delta(\mathrm{ppm}): 12.5,18.3,19.6$ [3CH $\left.3(\mathrm{Pbf})\right], 26.7$ [C $\left.\mathrm{C}_{\gamma}(\mathrm{Arg})\right], 28.6$

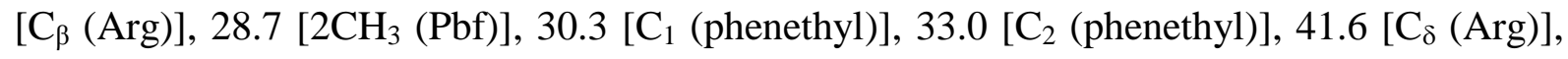
$43.6\left[\mathrm{CH}_{2}(\mathrm{Pbf})\right], 45.3\left(\mathrm{NHC}\left(\mathrm{NH}-\mathrm{CH}_{2}\right)=\mathrm{N}\right), 48.1\left(\mathrm{C}_{6}\right), 50.2\left(1-\mathrm{CH}_{2}\right), 51.0\left(\mathrm{C}_{5}\right), 51.5\left(4-\mathrm{CH}_{2}\right)$, $61.9\left(\mathrm{C}_{3}\right), 86.9,117.5,125.2$ [3C (Pbf)], 126.6, 127.9-129.9 (20CH, Ph), 142.7 and 139.9 (4C, $\mathrm{Ph}), 132.7,135.6,138.7,158.7$ [4C (Pbf)], 155.9 [NHC(NH-Bn)=N)], $170.5\left(\mathrm{C}_{2}\right) ; \mathrm{ES}-\mathrm{MS} \mathrm{m} / \mathrm{z}$ 
$827.04[\mathrm{M}+1]^{+}$; Anal. calcd. for $\mathrm{C}_{50} \mathrm{H}_{59} \mathrm{~N}_{5} \mathrm{O}_{4} \mathrm{~S}: \mathrm{C}, 72.70 ; \mathrm{H}, 7.20 ; \mathrm{N}, 8.48$. Found: C, 72.89; $\mathrm{H}, 7.31 ; \mathrm{N}, 8.25$.

\subsection{4. (5S,3S)-1,4-Dibenzyl-3-(3-(3-benzyl-2-((2,2,4,6,7-pentamethyl-2,3-dihydro- benzofuran-5-yl)sulfonyl)-guanidino)propyl)-5-phenethyl-2-oxopiperazine [(S)-41a]}

Amorphous solid (24 mg, $14 \%)$ ); HPLC-MS [Sunfire $\mathrm{C}_{18}(4.6 \times 50 \mathrm{~mm}, 3.5 \mu \mathrm{m}), 10$ $100 \%$ gradient of solvent $\mathrm{A}$ in $\mathrm{B}, 5 \mathrm{~min}] t_{\mathrm{R}} 5.63(70 \%)$ and $5.84(30 \%) \mathrm{min} ;{ }^{1} \mathrm{H}$ NMR [500 $\left.\mathrm{MHz},\left(\mathrm{CD}_{3}\right)_{2} \mathrm{CO}\right] \delta(\mathrm{ppm}): 1.41$ and 1.43 [2s, 6H, 2CH $\left.3(\mathrm{Pbf})\right], 1.54-1.76[\mathrm{~m}, 4 \mathrm{H}, \beta-\mathrm{H}$ and $\gamma-$ $\mathrm{H}(\mathrm{Arg})], 1.57$ and 1.82 [2m, 2H 1-H (phenethyl)], 2.01 [s, 3H, $\mathrm{CH}_{3}$ (Pbf)], 2.45 [s, 3H, $\mathrm{CH}_{3}$ (Pbf)], 2.51 [s, 3H, $\mathrm{CH}_{3}$ (Pbf)], 2.47 [m, 2H, 2-H (phenethyl)], 2.83 (m, 1H, 5-H), 2.95 [s, 2H, $\mathrm{CH}_{2}$ (Pbf)], 3.02-3.16 [m, 2H, $\delta-\mathrm{H}$ (Arg)], 3.18-3.28 (m, 2H, 3-H and 6-H), 3.48 and $3.51(2 \mathrm{~d}$, $1 \mathrm{H}, \mathrm{J}=5$ and $13 \mathrm{~Hz}, 6-\mathrm{H}), 3.67,3.72$ and $3.79\left(3 \mathrm{~d}, 2 \mathrm{H}, \mathrm{J}=14 \mathrm{~Hz}, 4-\mathrm{CH}_{2}\right), 4.42$ [d, 2H, J = 6 $\left.\mathrm{Hz}, \mathrm{NHC}\left(\mathrm{NH}-\mathrm{CH}_{2}\right)=\mathrm{N}\right], 4.57\left(\mathrm{~s}, 2 \mathrm{H}, 1-\mathrm{CH}_{2}\right), 7.08-7.35(\mathrm{~m}, 2 \mathrm{H}, \mathrm{Ph}) ;{ }^{13} \mathrm{C} \mathrm{NMR}[125 \mathrm{MHz}$, $\left.\left(\mathrm{CD}_{3}\right)_{2} \mathrm{CO}\right] \delta(\mathrm{ppm}): 12.5,18.3,19.5\left[3 \mathrm{CH}_{3}(\mathrm{Pbf})\right], 26.7\left[\mathrm{C}_{\gamma}(\mathrm{Arg})\right], 28.7$ [2 $\left.\mathrm{CH}_{3}(\mathrm{Pbf})\right], 31.1$

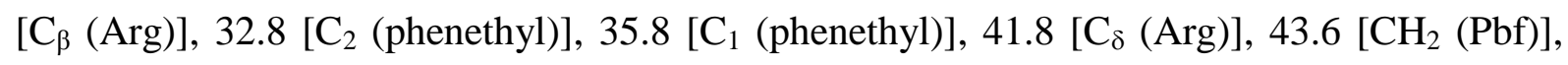
$45.3\left(\mathrm{NHC}\left(\mathrm{NH}-\mathrm{CH}_{2}\right)=\mathrm{N}\right), 48.0\left(\mathrm{C}_{6}\right), 50.2\left(1-\mathrm{CH}_{2}\right), 57.5$ and $57.7\left(\mathrm{C}_{5}\right), 59.3\left(4-\mathrm{CH}_{2}\right), 64.2$ $\left(\mathrm{C}_{3}\right), 86.9,117.5,125.2$ [3C (Pbf)], 126.5, 127.9-129.9 (20CH, Ph), 140.4, 140.5 and 142.8 $(4 \mathrm{C}, \mathrm{Ph}), 132.8,135.6,138.7,158.8$ [4C (Pbf)], 155.9 [NHC(NH-Bn)=N)], $171.1\left(\mathrm{C}_{2}\right)$; ESMS m/z $827.04[\mathrm{M}+1]^{+}$; Anal. calcd. for $\mathrm{C}_{50} \mathrm{H}_{59} \mathrm{~N}_{5} \mathrm{O}_{4} \mathrm{~S}: \mathrm{C}, 72.70 ; \mathrm{H}, 7.20 ; \mathrm{N}, 8.48$. Found: C, $72.87 ; \mathrm{H}, 7.33 ; \mathrm{N}, 8.31$.

\subsection{5. (5R,3S)-1,4-Dibenzyl-5-(2-(1-(tert-butoxycarbonyl)-indol-3-yl)ethyl-3-(3-(2-}

((2,2,4,6,7-pentamethyl-2,3-dihydrobenzofuran-5-yl)sulfonyl)guanidino)propyl)-2oxopiperazine [(R)-42a]

White solid (40 mg, $22 \%)$; $[\alpha]_{\mathrm{D}}{ }^{20}-8.4\left(c 1, \mathrm{CHCl}_{3}\right)$; Mp: 106-108 ${ }^{\circ} \mathrm{C}$; HPLC-MS [Sunfire $\mathrm{C}_{18}(4.6 \times 50 \mathrm{~mm}, 3.5 \mu \mathrm{m}), 80-100 \%$ gradient of solvent $\mathrm{A}$ in $\left.\mathrm{B}, 5 \mathrm{~min}\right] t_{\mathrm{R}} 4.06 \mathrm{~min} ;{ }^{1} \mathrm{H} \mathrm{NMR}$ (400 MHz, $\left.\mathrm{CDCl}_{3}\right) \delta$ (ppm): 1.44 [s, 6H, 2CH $\left.(\mathrm{Pbf})\right], 1.67$ (s, 9H, Boc), 1.55-2.03 [m, 6H, 1$\mathrm{H}$ (phenethyl), $\beta-\mathrm{H}$ and $\gamma-\mathrm{H}$ (Arg)], 2.07 [s, 3H, $\mathrm{CH}_{3}$ (Pbf)], 2.51 [s, 3H, $\mathrm{CH}_{3}$ (Pbf)], 2.57 [s, $3 \mathrm{H}, \mathrm{CH}_{3}$ (Pbf)], 2.47-2.62 and 2.64-2.78 [2m, 2H, 2-H (phenethyl)], 2.90 [s, 2H, $\mathrm{CH}_{2}$ (Pbf)], 2.85-3.04 [m, 2H, $\delta-\mathrm{H}(\mathrm{Arg})], 3.05-3.31(\mathrm{~m}, 4 \mathrm{H}, 3-\mathrm{H}, 5-\mathrm{H}, 6-\mathrm{H}), 3.38$ and $3.81(2 \mathrm{~d}, 2 \mathrm{H}, \mathrm{J}=$ $\left.13 \mathrm{~Hz}, 4-\mathrm{CH}_{2}\right), 4.42$ and $4.69\left(2 \mathrm{~d}, 2 \mathrm{H}, \mathrm{J}=14 \mathrm{~Hz}, 1-\mathrm{CH}_{2}\right), 6.04$ and 6.19 [2br s, 3H, $\left.\mathrm{NHC}\left(\mathrm{NH}_{2}\right)=\mathrm{N}\right], 7.12-7.24(\mathrm{~m}, 13 \mathrm{H}, \mathrm{Ar}), 7.32$ [d, 1H, J = 7.5 Hz, 7-H, (Ind)], 8.01 [br s, 1H, 4-H, (Ind)]; ${ }^{13} \mathrm{C}$ NMR (100 MHz, $\left.\mathrm{CDCl}_{3}\right) \delta$ (ppm): 12.6, 18.0, $19.4\left[3 \mathrm{CH}_{3}(\mathrm{Pbf})\right], 21.7\left[\mathrm{C}_{2}\right.$ 
(phenethyl)], $25.7\left[\mathrm{C}_{\gamma}(\mathrm{Arg})\right], 28.4\left[3 \mathrm{CH}_{3}(\mathrm{Boc})\right], 28.7\left[2 \mathrm{CH}_{3}(\mathrm{Pbf})\right], 29.8\left[\mathrm{C}_{\beta}(\mathrm{Arg})\right], 31.9\left[\mathrm{C}_{1}\right.$ (phenethyl)], $40.6\left[\mathrm{C}_{\delta}(\mathrm{Arg})\right], 43.3\left[\mathrm{CH}_{2}(\mathrm{Pbf})\right], 47.5\left(\mathrm{C}_{6}\right), 50.2\left(1-\mathrm{CH}_{2}\right), 50.4\left(\mathrm{C}_{5}\right), 51.3(4-$ $\left.\mathrm{CH}_{2}\right), 61.1\left(\mathrm{C}_{3}\right), 83.8$ (C, Boc), 86.3, 117.4, 124.6 [3C (Pbf)], 115.4, 118.9, 120.0, 122.5, 124.5, 127.6-129.0, 130.3, 135.5 (15 CH, Ar), 130.3, 133.2 (2C, Ind), 136.6, 138.4 (2C, Ph), 132.3, 135.6, 138.3, 158.6 [4C (Pbf)], 149.9 (CO, Boc), $156.2\left[\mathrm{C}\left(\mathrm{NHC}\left(\mathrm{NH}_{2}\right)=\mathrm{N}\right)\right], 171.0$ $\left(\mathrm{C}_{2}\right)$; ES-MS m/z 876.11 [M+1] $]^{+}$; Anal. calcd. for $\mathrm{C}_{50} \mathrm{H}_{62} \mathrm{~N}_{6} \mathrm{O}_{6} \mathrm{~S}: \mathrm{C}, 68.62 ; \mathrm{H}, 7.14 ; \mathrm{N}, 9.60$. Found: C, 68.92; H, 7.25; N, 9.52.

4.13.6. (5S,3S)-1,4-Dibenzyl-5-(2-(1-(tert-butoxycarbonyl)-indol-3-yl)ethyl-3-(3-(2-

((2,2,4,6,7-pentamethyl-2,3-dihydrobenzofuran-5-yl)sulfonyl)guanidino)propyl)-2oxopiperazine [(S)-42a]

White solid $(77 \mathrm{mg}, 42 \%)) ;[\alpha]_{\mathrm{D}}{ }^{20}+7.7\left(c 1, \mathrm{CHCl}_{3}\right)$; Mp: 96-98 ${ }^{\circ} \mathrm{C}$; HPLC-MS [Sunfire $\mathrm{C}_{18}(4.6 \times 50 \mathrm{~mm}, 3.5 \mu \mathrm{m}), 40-100 \%$ gradient of solvent $\mathrm{A}$ in $\left.\mathrm{B}, 5 \mathrm{~min}\right] t_{\mathrm{R}} 6.43 \mathrm{~min} ;{ }^{1} \mathrm{H}$ NMR (400 MHz, $\left.\mathrm{CDCl}_{3}\right) \delta(\mathrm{ppm}): 1.46$ [s, 6H, 2CH 3 (Pbf)], 1.68 (s, 9H, Boc), 1.53-1.99 [m, 6H, 1$\mathrm{H}$ (phenethyl), $\beta-\mathrm{H}$ and $\gamma-\mathrm{H}$ (Arg)], 2.08 [s, 3H, $\mathrm{CH}_{3}$ (Pbf)], 2.53 [s, 3H, $\mathrm{CH}_{3}$ (Pbf)], 2.59 [s, $\left.3 \mathrm{H}, \mathrm{CH}_{3}(\mathrm{Pbf})\right], 2.47-2.56$ [m, 2H, 2-H (phenethyl)], 2.77 (m, 1H, 5-H), 2.79 [s, 2H, $\mathrm{CH}_{2}$ (Pbf)], 2.96 [m, 2H, $\delta-\mathrm{H}(\mathrm{Arg})], 3.08$ (dd, 1H, J =8.5 and $13 \mathrm{~Hz}, 6-\mathrm{H}), 3.19-3.28$ (m, 2H, 3-H, and 6-H), 3.60 and $3.62\left(2 \mathrm{~d}, 2 \mathrm{H}, \mathrm{J}=14 \mathrm{~Hz}, 4-\mathrm{CH}_{2}\right), 4.43$ and $4.49(2 \mathrm{~d}, 2 \mathrm{H}, \mathrm{J}=14 \mathrm{~Hz}, 1-$ $\left.\mathrm{CH}_{2}\right), 6.01$ and 6.12 [2br s, 3H, NHC( $\left.\left.\mathrm{NH}_{2}\right)=\mathrm{N}\right], 7.05-7.23(\mathrm{~m}, 14 \mathrm{H}, \mathrm{Ar}), 7.99$ [br s, 1H, 4-H, (Ind)]; ${ }^{13} \mathrm{C}$ NMR (100 MHz, $\left.\mathrm{CDCl}_{3}\right) \delta(\mathrm{ppm}): 12.6,18.0,19.4\left[3 \mathrm{CH}_{3}(\mathrm{Pbf})\right], 21.3\left[\mathrm{C}_{2}\right.$ (phenethyl)], $25.9\left[\mathrm{C}_{\gamma}(\mathrm{Arg})\right], 28.3\left[3 \mathrm{CH}_{3}(\mathrm{Boc})\right], 28.6\left[2 \mathrm{CH}_{3}(\mathrm{Pbf})\right], 29.8\left[\mathrm{C}_{\beta}(\mathrm{Arg})\right], 30.4\left[\mathrm{C}_{1}\right.$ (phenethyl)], $40.8\left[\mathrm{C}_{\delta}(\mathrm{Arg})\right], 43.3\left[\mathrm{CH}_{2}(\mathrm{Pbf})\right], 47.4\left(\mathrm{C}_{6}\right), 50.1\left(1-\mathrm{CH}_{2}\right), 56.8\left(\mathrm{C}_{5}\right), 58.8(4-$ $\left.\mathrm{CH}_{2}\right), 63.6\left(\mathrm{C}_{3}\right), 83.7$ (C, Boc), 86.3, 117.4, 124.5 [3C (Pbf)], 115.3, 118.8, 120.0, 122.3, 122.5, 127.5-128.9 (15 CH, Ar), 130.3, 133.3 (2C, Ind), 135.5, 138.9 (2C, Ph), 132.3, 136.4, 138.4, 158.6 [4C (Pbf)], 149.8 (CO, Boc), 156.2 [C $\left.\left(\mathrm{NHC}\left(\mathrm{NH}_{2}\right)=\mathrm{N}\right)\right], 171.2\left(\mathrm{C}_{2}\right) ; \mathrm{ES}-\mathrm{MS} \mathrm{m} / \mathrm{z}$ $876.11[\mathrm{M}+1]^{+}$; Anal. calcd. for $\mathrm{C}_{50} \mathrm{H}_{62} \mathrm{~N}_{6} \mathrm{O}_{6} \mathrm{~S}: \mathrm{C}, 68.62 ; \mathrm{H}, 7.14 ; \mathrm{N}, 9.60$. Found: C, 68.82; H, 7.22; N, 9.47.

4.13.7. (5R,3S)-1,4-Dibenzyl-5-(2-(1-(tert-butoxycarbonyl)-indol-3-yl)ethyl-3-(3-(3-benzyl-2((2,2,4,6,7-pentamethyl-2,3-dihydrobenzofuran-5-yl)sulfonyl)guanidino)propyl)-2oxopiperazine [(R)-44a]

Amorphous solid (20 mg, $10 \%)$ ); HPLC-MS [Sunfire $\mathrm{C}_{18}(4.6 \times 50 \mathrm{~mm}, 3.5 \mu \mathrm{m})$, isocratic $95 \%$ of solvent $\mathrm{A}$ in $5 \%$ of $\mathrm{B}] t_{\mathrm{R}} 2.32(73 \%)$ and $2.47(27 \%) \mathrm{min} ;{ }^{1} \mathrm{H}$ NMR [500 MHz, 
$\left.\left(\mathrm{CD}_{3}\right)_{2} \mathrm{CO}\right] \delta$ (ppm): 1.39 and 1.42 [2s, 6H, 2CH $\left.3(\mathrm{Pbf})\right], 1.64$ (s, 9H, Boc), 1.55-2.03 [m, 6H, 1-H (phenethyl), $\beta$-H and $\gamma$-H (Arg)], 1.99 [s, 3H, $\mathrm{CH}_{3}$ (Pbf)], 2.45 [s, 3H, $\mathrm{CH}_{3}$ (Pbf)], 2.51 [s, 3H, $\mathrm{CH}_{3}$ (Pbf)], 2.59-2.78 [m, 2H, 2-H (phenethyl)], 2.92 [s, 2H, $\left.\mathrm{CH}_{2}(\mathrm{Pbf})\right], 3.06$ [m, 2H, $\delta$-H (Arg)], 3.13 (m, 1H, 3-H), 3.20 and 3.38 (2m, 2H, 6-H), 3.34 (m, 1H, 5-H), 3.41 (d, 1H, J $\left.=14 \mathrm{~Hz}, 4-\mathrm{CH}_{2}\right), 3.91\left(\mathrm{~m}, 1 \mathrm{H}, 4-\mathrm{CH}_{2}\right), 4.42\left[\mathrm{~d}, 2 \mathrm{H}, \mathrm{J}=6 \mathrm{~Hz}, \mathrm{NHC}\left(\mathrm{NH}-\mathrm{CH}_{2}\right)=\mathrm{N}\right], 4.53,4.55$, 4.60 and $4.65\left(4 \mathrm{~d}, 2 \mathrm{H}, \mathrm{J}=14 \mathrm{~Hz}, 1-\mathrm{CH}_{2}\right), 7.12-7.37$ (m, 18H, Ar), 7.49 and $7.53(2 \mathrm{~d}, 1 \mathrm{H}, \mathrm{J}=$ $8 \mathrm{~Hz}, 7-\mathrm{H}$, (Ind)], 8.12 [d, 1H, J = 7.5 Hz, 4-H, (Ind)]; $\left.{ }^{13} \mathrm{C} \mathrm{NMR} \mathrm{[125} \mathrm{MHz,}\left(\mathrm{CD}_{3}\right)_{2} \mathrm{CO}\right] \delta$ (ppm): 12.4, 18.1, $19.4\left[3 \mathrm{CH}_{3}\right.$ (Pbf)], $22.0\left[\mathrm{C}_{2}\right.$ (phenethyl)], $24.7\left[\mathrm{C}_{\gamma}(\mathrm{Arg})\right], 28.1\left[2 \mathrm{CH}_{3}\right.$ (Pbf)], $28.5\left[3 \mathrm{CH}_{3}(\mathrm{Boc})\right], 27.6\left[\mathrm{C}_{\beta}(\mathrm{Arg})\right], 28.4\left[\mathrm{C}_{1}\right.$ (phenethyl)], $41.4\left[\mathrm{C}_{\delta}(\mathrm{Arg})\right], 43.4\left[\mathrm{CH}_{2}\right.$ $(\mathrm{Pbf})], 47.9\left(\mathrm{C}_{6}\right), 50.1\left(1-\mathrm{CH}_{2}\right), 51.2\left(\mathrm{C}_{5}\right), 51.5\left(4-\mathrm{CH}_{2}\right), 61.8$ and $61.9\left(\mathrm{C}_{3}\right), 83.9(\mathrm{C}, \mathrm{Boc})$, 86.7, 117.3, 124.9 [3C (Pbf)], 115.8, 119.8, 121.2, 123.0, 124.9, 125.1, 127.7-129.6 (20 CH, Ar), 131.2, 135.5 (2C, Ind), 136.3, 138.5, 139.7 (3C, Ph), 132.6, 135.4, 138.7, 158.7 [4C (Pbf)], 150.1 (CO, Boc), 155.7 and 156.5 [NHC(NH-Bn)=N)], $170.6\left(\mathrm{C}_{2}\right)$; ES-MS m/z 965.96 $[\mathrm{M}+1]^{+}$; Anal. calcd. for $\mathrm{C}_{57} \mathrm{H}_{68} \mathrm{~N}_{6} \mathrm{O}_{6} \mathrm{~S}: \mathrm{C}, 70.93 ; \mathrm{H}, 7.10 ; \mathrm{N}, 8.71$. Found: C, 70.82; H, 7.14; N, 8.82.

4.13.8. (5S,3S)-1,4-Dibenzyl-5-(2-(1-(tert-butoxycarbonyl)-indol-3-yl)ethyl-3-(3-(3-benzyl-2-

((2,2,4,6,7-pentamethyl-2,3-dihydrobenzofuran-5-yl)sulfonyl)guanidino)propyl)-2oxopiperazine [(S)-44a]

Amorphous solid (16 mg, $8 \%)$ ); HPLC-MS [Sunfire $\mathrm{C}_{18}(4.6 \times 50 \mathrm{~mm}, 3.5 \mu \mathrm{m})$, isocratic $95 \%$ of solvent $\mathrm{A}$ in $5 \%$ of $\mathrm{B}] t_{\mathrm{R}} 2.36(75 \%)$ and $2.52(25 \%) \mathrm{min} ;{ }^{1} \mathrm{H}$ NMR [500 MHz, $\left.\left(\mathrm{CD}_{3}\right)_{2} \mathrm{CO}\right] \delta(\mathrm{ppm}): 1.41\left[\mathrm{~s}, 6 \mathrm{H}, 2 \mathrm{CH}_{3}(\mathrm{Pbf})\right], 1.65$ (s, 9H, Boc), 1.67-1.91 [m, 6H, 1-H (phenethyl), $\beta-\mathrm{H}$ and $\gamma-\mathrm{H}$ (Arg)], 2.00 [s, 3H, $\left.\mathrm{CH}_{3}(\mathrm{Pbf})\right], 2.43$ [s, 3H, $\left.\mathrm{CH}_{3}(\mathrm{Pbf})\right], 2.51$ [s, 3H, $\mathrm{CH}_{3}$ (Pbf)], 2.58 [m, 2H, 2-H (phenethyl)], 2.91 [s, 2H, $\mathrm{CH}_{2}$ (Pbf)], 2.96 (m, 1H, 5-H), 3.12 [m, 2H, $\delta-\mathrm{H}(\mathrm{Arg})], 3.29(\mathrm{~m}, 1 \mathrm{H}, 3-\mathrm{H}), 3.27$ and $3.59(2 \mathrm{~m}, 2 \mathrm{H}, 6-\mathrm{H}), 3.78\left(\mathrm{~m}, 1 \mathrm{H}, 4-\mathrm{CH}_{2}\right)$, 4.42 and $4.56\left[2 \mathrm{~d}, 2 \mathrm{H}, \mathrm{J}=5.5 \mathrm{~Hz}, \mathrm{NHC}\left(\mathrm{NH}-\mathrm{CH}_{2}\right)=\mathrm{N}\right], 4.51$ and $4.67(2 \mathrm{~d}, 2 \mathrm{H}, \mathrm{J}=14.5 \mathrm{~Hz}, 1-$ $\mathrm{CH}_{2}$ ), 7.12-7.37 (m, 18H, Ar), $7.41(\mathrm{~d}, 1 \mathrm{H}, \mathrm{J}=7.5 \mathrm{~Hz}, 7-\mathrm{H},(\mathrm{Ind})], 8.09$ [d, 1H, J = 7.5 Hz, 4$\mathrm{H},\left(\right.$ Ind)]; ${ }^{13} \mathrm{C} \mathrm{NMR}\left[125 \mathrm{MHz},\left(\mathrm{CD}_{3}\right)_{2} \mathrm{CO}\right] \delta$ (ppm): 12.2, 18.0, 19.3 [3 $\left.\mathrm{CH}_{3}(\mathrm{Pbf})\right], 21.9\left[\mathrm{C}_{2}\right.$ (phenethyl)], $26.7\left[\mathrm{C}_{\gamma}(\mathrm{Arg})\right], 28.0\left[2 \mathrm{CH}_{3}(\mathrm{Pbf})\right], 28.3\left[3 \mathrm{CH}_{3}(\mathrm{Boc})\right], 31.0\left[\mathrm{C}_{\beta}(\mathrm{Arg})\right], 33.5\left[\mathrm{C}_{1}\right.$ (phenethyl)], $41.8\left[\mathrm{C}_{\delta}(\mathrm{Arg})\right], 43.3\left[\mathrm{CH}_{2}(\mathrm{Pbf})\right], 45.3\left(1-\mathrm{CH}_{2}\right), 48.0\left(\mathrm{C}_{6}\right), 57.6\left(\mathrm{C}_{5}\right), 59.2(4-$ $\left.\mathrm{CH}_{2}\right), 64.2\left(\mathrm{C}_{3}\right), 84.0$ (C, Boc), 86.9, 117.5, 125.0 [3C (Pbf)], 115.9, 119.9, 121.4, 123.2, 125.0, 125.2, 127.9-129.8 (20 CH, Ar), 131.3, 135.7, 135.1 (2C, Ind), 136.4, 138.4, 140.5 (3C, $\mathrm{Ph}), 132.8,135.6,138.7,158.8$ [4C (Pbf)], 150.3 (CO, Boc), 155.9 and 156.5 [NHC(NH- 
$\mathrm{Bn})=\mathrm{N})$ ], $171.2\left(\mathrm{C}_{2}\right)$; ES-MS m/z $965.96[\mathrm{M}+1]^{+}$; Anal. calcd. for $\mathrm{C}_{57} \mathrm{H}_{68} \mathrm{~N}_{6} \mathrm{O}_{6} \mathrm{~S}: \mathrm{C}, 70.93 ; \mathrm{H}$, 7.10; N, 8.71. Found: C, 71.07; H, 7.19; N, 8.60.

\subsection{Platelet aggregation inhibition assay}

Whole blood was obtained from human volunteers who were not taking any platelet altering drugs for two weeks prior to donation. Blood was collected by venous puncture into $2.7 \mathrm{~mL}$ vacutainer tubes containing $3.2 \%$ buffered sodium citrate. Blood was centrifuged at $250 \times \mathrm{g}$ for $7 \mathrm{~min}$ to obtain platelet rich plasma (PRP). After removal of PRP, the blood was recentrifuged at $900 \times \mathrm{g}$ for $10 \mathrm{~min}$ to obtain platelet poor plasma (PPP). The PPP was used as a reference in the optical aggregation and as a diluent to achieve a final platelet concentration of 200.000 platelet $/ \mu \mathrm{L}$ in PRP. Tests were performed in an optical aggregometer (Chrono-Log Model 440 Four Channel). Briefly, a $0.5 \mathrm{~mL}$ sample of diluted PRP was added to a glass cuvette and incubated with either vehicle (DMSO solution) or tested compound, at a 0.1 $\mathrm{mg} / \mathrm{mL}$ concentration, for $5 \mathrm{~min}$ at $37^{\circ} \mathrm{C}$. At the beginning of each experiment, aggregation response to SFLLRN $(30 \mu \mathrm{M})$ was evaluated and the maximum aggregation value at the end of $5 \mathrm{~min}$ was recorded. Aggregation response to SFLLRN plus compound was recorded and compared to control (SFLLRN/vehicle) to determine the \% of inhibition. Each compound was tested twice and the results are the mean of the two assays.

\subsection{Cytotoxicity assays}

A colorimetric assay, using the sulforhodamine B (SRB) reaction, was adapted for a quantitative measurement of cell growth and viability, following the technique described by Skehan, P. A. et al. [59]. Cells (MDA-MB-231, A549 and HT-29) were seeded in 96 well microtiter plates, at $5 \times 10^{3}$ cells per well in aliquots of $195 \mu \mathrm{L}$ of RPMI medium, and they were allowed to attach to the plate surface by growing in drug free medium for $18 \mathrm{~h}$. Afterwards, samples were added in aliquots of $5 \mu \mathrm{L}$ [dissolved in (3:7) DMSO/ $\mathrm{H}_{2} \mathrm{O}$ ]. After 48 h exposure, cells were fixed by adding $50 \mu \mathrm{L}$ of cold $50 \%$ (wt/vol) trichloroacetic acid, and incubating at $4{ }^{\circ} \mathrm{C}$ for $60 \mathrm{~min}$. Then, the plates were washed with deionized $\mathrm{H}_{2} \mathrm{O}$ and dried. $100 \mu \mathrm{L}$ of SRB solution $(0.4 \% \mathrm{wt} / \mathrm{vol}$ in $1 \%$ acetic acid) was added to each microtiter well and these were incubated at room temperature for $10 \mathrm{~min}$. Unbound SRB was removed by washing with $1 \%$ acetic acid, the plates were air dried, and the bound stain was solubilized with Tris buffer. Optical densities were read on an automated spectrophotometer plate reader at a single wavelength of $490 \mathrm{~nm}$. Data analysis was automatically generated by the high 
throughput screening LIMS implemented at the laboratory. The three response parameters $\mathrm{GI}_{50}$ (50\% cell growth inhibition), $\mathrm{LC}_{50}$ (50\% lethal concentration), and TGI (total growth inhibition) were extracted from concentration-response curves by linear interpolation, according to the National Cancer Institute (NCI) protocols [60].

\section{Acknowledgements}

This work was supported by the Spanish Ministerio de Ciencia e Innovación grant SAF2009-09323. Á. M. V. held a FPU fellowship from the Ministerio de Educación y Ciencia. P. V.-A. held a FPI fellowship from the Ministerio de Educación y Ciencia. F. T. held a JAEdoc research contract from the CSIC. M. Á. F.-I. held a Juan de la Cierva research contract from the Ministerio de Educación y Ciencia. The antitumor screening was carried out at Pharma Mar, S.A.

Supplementary Content Available: Synthesis procedure for the aldehyde 31 and ${ }^{1} \mathrm{H}$ and ${ }^{13} \mathrm{C}$ NMR spectra of all new compounds.

\section{References}

[1] S.R. Macfarlane, M.J. Seatter, T. Kanke, G.D. Hunter, R. Plevin, Proteinase-activated receptors, Pharmacol. Rev., 53 (2001) 245-282.

[2] L. Martorell, J. Martínez-González, C. Rodríguez, M. Gentile, O. Calvayrac, L. Badimon, Thrombin and protease-activated receptors (PARs) in atherothrombosis, Thromb. Haemost., 99 (2008) 305-315.

[3] R. Shah, Protease-activated receptors in cardiovascular health and diseases, Am. Heart J., 157 (2009) 253 262.

[4] R. Ramachandran, F. Noorbakhsh, K. Defea, M.D. Hollenberg, Targeting proteinase-activated receptors: therapeutic potential and challenges, Nat. Rev. Drug Discov., 11 (2012) 69-86.

[5] V.S. Ossovskaya, N.W. Bunnett, Protease-activated receptors: contribution to physiology and disease, Physiol. Rev., 84 (2004) 579-621.

[6] E. Sokolova, G. Reiser, Prothrombin/thrombin and the thrombin receptors PAR-1 and PAR-4 in the brain: localization, expression and participation in neurodegenerative diseases, Thromb. Haemost., 100 (2008) 576-581.

[7] W. Luo, Y. Wang, G. Reiser, The role of thrombin and thrombin receptors in the brain, in: M.E. Maragoudakis, N.E. Tsopanoglou (Eds.) Thrombin: Physiology and Disease, Springer, New York, 2009, pp. 133-159.

[8] Y. Hashimotodani, T. Ohno-Shosaku, M. Yamazaki, K. Sakimura, M. Kano, Neuronal protease-activated receptor 1 drives synaptic retrograde signaling mediated by the endocannabinoid 2-arachidonoylglycerol, J. Neurosci., 31 (2011) 3104-3109.

[9] A.G. Almonte, L.H. Qadri, F.A. Sultan, J.A. Watson, D.J. Mount, G. Rumbaugh, J.D. Sweatt, Proteaseactivated receptor-1 modulates hippocampal memory formation and synaptic plasticity, J. Neurochem., 124 (2013) 109-122.

[10] N. Maggio, C. Cavaliere, M. Papa, I. Blatt, J. Chapman, M. Segal, Thrombin regulation of synaptic transmission: Implications for seizure onset, Neurobiol. Dis., 50 (2013) 171-178. 
[11] M. Steinhoff, J. Buddenkotte, V. Shpacovitch, A. Rattenholl, C. Moormann, N. Vergnolle, T.A. Luger, M.D. Hollenberg, Proteinase-activated receptors: transducers of proteinase-mediated signaling in inflammation and immune response, Endocr. Rev., 26 (2005) 1-43.

[12] R. Ramachandran, M. El-Daly, M. Saifeddine, M.D. Hollenberg, Thrombin: To PAR or not to PAR, and the regulation of inflamation, in: M.E. Maragoudakis, N.E. Tsopanoglou (Eds.) Thrombin: Physiology and Disease

Springer, New York, 2009, pp. 19-46.

[13] P. Arora, T.K. Ricks, J. Trejo, Protease-activated receptor signalling, endocytic sorting and dysregulation in cancer, J. Cell Sci., 120 (2007) 921-928.

[14] E. Camerer, Protease signaling in tumor progression, Thromb. Res., 120 Suppl 2 (2007) S75-S81.

[15] K.S. Borensztajn, C.A. Spek, Protease-activated receptors, apoptosis and tumor growth, Pathophysiol. Haemost. Thromb., 36 (2008) 137-147.

[16] B. Kobrinsky, S. Karpatkin, The role of thrombin in tumor biology, in: M.E. Maragoudakis, N.E. Tsopanoglou (Eds.) Thrombin: Physiology and Disease, Springer, New York, 2009, pp. 161-172.

[17] C.S.B. Veiga, T.C. Carneiro-Lobo, C.J.B.P. Coelho, S.M.F. Carvalho, R.C. Maia, F.C. Vasconcelos, E. Abdelhay, A.L. Mencalha, A.F. Ferreira, F.A. Castro, R.Q. Monteiro, Increased expression of proteaseactivated receptor 1 (PAR-1) in human leukemias, Blood Cells Mol. Dis., 46 (2011) 230-234.

[18] Z. Salah, B. Uziely, M. Jaber, M. Maoz, I. Cohen, T. Hamburger, B. Maly, T. Peretz, R. Bar-Shavit, Regulation of human protease-activated receptor 1 (hPar1) gene expression in breast cancer by estrogen, FASEB J., 26 (2012) 2031-2042.

[19] M.T. García-López, M. Gutiérrez-Rodríguez, R. Herranz, Thrombin-Activated Receptors: Promising Targets for Cancer Therapy?, Curr Med Chem, 17 (2010) 109-128.

[20] C. Chen, B.E. Maryanoff, P. Andrade-Gordon, Thrombin receptor modulators: Medicinal chemistry, biological evaluation, and clinical applications, in: M.E. Maragoudakis, N.E. Tsopanoglou (Eds.) Thrombin: Physiology and Disease, Springer, New York, 2009, pp. 205-236.

[21] C. Tellez, M. Bar-Eli, Role and regulation of the thrombin receptor (PAR-1) in human melanoma, Oncogene, 22 (2003) 3130-3137.

[22] S. Granovsky-Grisaru, S. Zaidoun, D. Grisaru, Y. Yekel, D. Prus, U. Beller, R. Bar-Shavit, The pattern of Protease Activated Receptor 1 (PAR1) expression in endometrial carcinoma, Gynecol. Oncol., 103 (2006) 802-806.

[23] X. Zhang, W. Wang, L.D. True, R.L. Vessella, T.K. Takayama, Protease-activated receptor-1 is upregulated in reactive stroma of primary prostate cancer and bone metastasis, Prostate, 69 (2009) 727-736.

[24] C. Rudroff, S. Seibold, R. Kaufmann, C.C. Zetina, K. Reise, U. Schafer, A. Schneider, M. Brockmann, J. Scheele, E.A. Neugebauer, Expression of the thrombin receptor PAR-1 correlates with tumour cell differentiation of pancreatic adenocarcinoma in vitro, Clin. Exp. Metastasis, 19 (2002) 181-189.

[25] S. Grisaru-Granovsky, Z. Salah, M. Maoz, D. Pruss, U. Beller, R. Bar-Shavit, Differential expression of protease activated receptor 1 (Par1) and pY397FAK in benign and malignant human ovarian tissue samples, Int. J. Cancer, 113 (2005) 372-378.

[26] D. Darmoul, V. Gratio, H. Devaud, T. Lehy, M. Laburthe, Aberrant expression and activation of the thrombin receptor protease-activated receptor-1 induces cell proliferation and motility in human colon cancer cells, Am. J. Pathol., 162 (2003) 1503-1513.

[27] P. Ghio, S. Cappia, G. Selvaggi, S. Novello, P. Lausi, G. Zecchina, M. Papotti, P. Borasio, G.V. Scagliotti, Prognostic role of protease-activated receptors 1 and 4 in resected stage IB non-small-cell lung cancer, Clin. Lung Cancer, 7 (2006) 395-400.

[28] X. Zhang, J.L. Hunt, D.P. Landsittel, S. Muller, K. Adler-Storthz, R.L. Ferris, D.M. Shin, Z.G. Chen, Correlation of protease-activated receptor-1 with differentiation markers in squamous cell carcinoma of the head and neck and its implication in lymph node metastasis, Clin. Cancer Res., 10 (2004) 8451-8459.

[29] H.C. Zhang, C.K. Derian, P. Andrade-Gordon, W.J. Hoekstra, D.F. McComsey, K.B. White, B.L. Poulter, M.F. Addo, W.M. Cheung, B.P. Damiano, D. Oksenberg, E.E. Reynolds, A. Pandey, R.M. Scarborough, B.E. Maryanoff, Discovery and optimization of a novel series of thrombin receptor (par-1) antagonists: potent, selective peptide mimetics based on indole and indazole templates, J Med Chem, 44 (2001) 10211024.

[30] S. Chackalamannil, Y. Xia, W.J. Greenlee, M. Clasby, D. Doller, H. Tsai, T. Asberom, M. Czarniecki, H.S. Ahn, G. Boykow, C. Foster, J. Agans-Fantuzzi, M. Bryant, J. Lau, M. Chintala, Discovery of potent orally active thrombin receptor (protease activated receptor 1) antagonists as novel antithrombotic agents, J. Med. Chem., 48 (2005) 5884-5887.

[31] S. Chackalamannil, Y. Wang, W.J. Greenlee, Z. Hu, Y. Xia, H.S. Ahn, G. Boykow, Y. Hsieh, J. Palamanda, J. Agans-Fantuzzi, S. Kurowski, M. Graziano, M. Chintala, Discovery of a novel, orally active himbacine- 
based thrombin receptor antagonist (SCH 530348) with potent antiplatelet activity, J Med Chem, 51 (2008) 3061-3064.

[32] Y. Shinohara, S. Goto, M. Doi, P. Jensen, Safety of the Novel Protease-Activated Receptor-1 Antagonist Vorapaxar in Japanese Patients with a History of Ischemic Stroke, J. Stroke Cerebrovasc. Dis., 21 (2012) 318-324.

[33] P. Tricoci, Z. Huang, C. Held, D.J. Moliterno, P.W. Armstrong, F. Van de Werf, H.D. White, P.E. Aylward, L. Wallentin, E. Chen, Y. Lokhnygina, J. Pei, S. Leonardi, T.L. Rorick, A.M. Kilian, L.H. Jennings, G. Ambrosio, C. Bode, A. Cequier, J.H. Cornel, R. Diaz, A. Erkan, K. Huber, M.P. Hudson, L. Jiang, J.W. Jukema, B.S. Lewis, A.M. Lincoff, G. Montalescot, J.C. Nicolau, H. Ogawa, M. Pfisterer, J.C. Prieto, W. Ruzyllo, P.R. Sinnaeve, R.F. Storey, M. Valgimigli, D.J. Whellan, P. Widimsky, J. Strony, R.A. Harrington, K.W. Mahaffey, Thrombin-receptor antagonist vorapaxar in acute coronary syndromes, N. Engl. J. Med., 366 (2012) 20-33.

[34] S. Goto, V. Serebruany, Vorapaxar: PAR1 receptor antagonist, antiplatelet therapy, Drugs Fut., 36 (2011) $101-113$

[35] T.K. Vu, D.T. Hung, V.I. Wheaton, S.R. Coughlin, Molecular cloning of a functional thrombin receptor reveals a novel proteolytic mechanism of receptor activation, Cell, 64 (1991) 1057-1068.

[36] B.D. Blackhart, L. Ruslim-Litrus, C.C. Lu, V.L. Alves, W. Teng, R.M. Scarborough, E.E. Reynolds, D. Oksenberg, Extracellular mutations of protease-activated receptor-1 result in differential activation by thrombin and thrombin receptor agonist peptide, Mol. Pharmacol., 58 (2000) 1178-1187.

[37] T.K. Vu, V.I. Wheaton, D.T. Hung, I. Charo, S.R. Coughlin, Domains specifying thrombin-receptor interaction, Nature, 353 (1991) 674-677.

[38] Y.M. Ayala, A.M. Cantwell, T. Rose, L.A. Bush, D. Arosio, E. Di Cera, Molecular mapping of thrombinreceptor interactions, Proteins, 45 (2001) 107-116.

[39] M.T. Nieman, A.H. Schmaier, Interaction of thrombin with PAR1 and PAR4 at the thrombin cleavage site, Biochemistry, 46 (2007) 8603-8610.

[40] S.L. Jacques, M. LeMasurier, P.J. Sheridan, S.K. Seeley, A. Kuliopulos, Substrate-assisted catalysis of the PAR1 thrombin receptor. Enhancement of macromolecular association and cleavage, J. Biol. Chem., 275 (2000) 40671-44068.

[41] T. Myles, B.F. Le Bonniec, S.R. Stone, The dual role of thrombin's anion-binding exosite-I in the recognition and cleavage of the protease-activated receptor 1, Eur. J. Biochem., 268 (2001) 70-77.

[42] W.F. Bahou, J.L. Kutok, A. Wong, C.L. Potter, B.S. Coller, Identification of a novel thrombin receptor sequence required for activation-dependent responses, Blood, 84 (1994) 4195-4202.

[43] T. Nanevicz, M. Ishii, L. Wang, M. Chen, J. Chen, C.W. Turck, F.E. Cohen, S.R. Coughlin, Mechanisms of thrombin receptor agonist specificity. Chimeric receptors and complementary mutations identify an agonist recognition site, J. Biol. Chem., 270 (1995) 21619-21625.

[44] P.S. Gandhi, Z. Chen, F.S. Mathews, E. Di Cera, Structural identification of the pathway of long-range communication in an allosteric enzyme, Proc. Natl. Acad. Sci. U S A, 105 (2008) 1832-1837.

[45] P.S. Gandhi, Z. Chen, E. Di Cera, Crystal structure of thrombin bound to the uncleaved extracellular fragment of PAR1, J. Biol. Chem., 285 (2010) 15393-15398.

[46] S. Seeley, L. Covic, S.L. Jacques, J. Sudmeier, J.D. Baleja, A. Kuliopulos, Structural basis for thrombin activation of a protease-activated receptor: inhibition of intramolecular liganding, Chem. Biol., 10 (2003) 1033-1041.

[47] P. Ventosa-Andrés, A.M. Valdivielso, I. Pappos, M.T. García-López, N.E. Tsopanoglou, R. Herranz, Design, synthesis and biological evaluation of new peptide-based ureas and thioureas as potential antagonists of the thrombin receptor PAR1, Eur. J. Med. Chem., 58 (2012) 98-111.

[48] S. Herrero, M.T. García-López, M. Latorre, E. Cenarruzabeitia, J. Del Río, R. Herranz, 2-Oxopiperazinebased gamma-turn conformationally constrained peptides: synthesis of CCK-4 analogues, J. Org. Chem., 67 (2002) 3866-3873.

[49] A.M. Valdivielso, P. Ventosa-Andrés, M.T. García-López, R. Herranz, M. Gutiérrez-Rodríguez, Synthesis and Regioselective Functionalization of Piperazin-2-ones Based on Phe-Gly Pseudodipeptides, Eur. J. Org. Chem., 2013 (2013) 155-161.

[50] C. Knox, V. Law, T. Jewison, P. Liu, S. Ly, A. Frolkis, A. Pon, K. Banco, C. Mak, V. Neveu, Y. Djoumbou, R. Eisner, A.C. Guo, D.S. Wishart, DrugBank 3.0: a comprehensive resource for 'omics' research on drugs, Nucleic Acids Res., 39 (2011) D1035-D1041.

[51] R. Herranz, M.L. Suárez-Gea, S. Vinuesa, M.T. García-López, Studies on the synthesis of cyanomethyleneamino pseudopeptides, J. Org. Chem., 58 (1993) 5186-5191.

[52] Y. Yamamoto, S. Kojima, Synthesis and chemistry of guanidine derivatives, in: S. Patai, Z. Rappoport (Eds.) The Chemistry of Amidines and Imidates, John Wiley \& Sons, Ltd., Chichester, 1991, pp. 485-526. 
[53] K. Kanamori, J.D. Roberts, A nitrogen-15 NMR study of the barriers to isomerization about guanidinium and guanidino carbon-nitrogen bonds in L-arginine, J. Am. Chem. Soc., 105 (1983) 4698-4701.

[54] W.X. Zhang, M. Nishiura, Z. Hou, Catalytic addition of amine N-H bonds to carbodiimides by halfsandwich rare-earth metal complexes: efficient synthesis of substituted guanidines through amine protonolysis of rare-earth metal guanidinates, Chem. Eur. J., 13 (2007) 4037-4051.

[55] J.-A. Fehrentz, B. Castro, An Efficient Synthesis of Optically Active $\alpha$-(t-Butoxycarbonylamino)-aldehydes from $\alpha$-Amino Acids, Synthesis, 1983 (1983) 676-678.

[56] E. Ko, J. Liu, L.M. Perez, G. Lu, A. Schaefer, K. Burgess, Universal Peptidomimetics, J. Am. Chem. Soc., 133 (2010) 462-477.

[57] M. Amatore, T.D. Beeson, S.P. Brown, D.W.C. MacMillan, Enantioselective Linchpin Catalysis by SOMO Catalysis: An Approach to the Asymmetric $\alpha$-Chlorination of Aldehydes and Terminal Epoxide Formation, Angew. Chem. Int. Ed., 48 (2009) 5121-5124.

[58] S. Rendler, D.W.C. MacMillan, Enantioselective Polyene Cyclization via Organo-SOMO Catalysis, J. Am. Chem. Soc., 132 (2010) 5027-5029.

[59] P. Skehan, R. Storeng, D. Scudiero, A. Monks, J. McMahon, D. Vistica, J.T. Warren, H. Bokesch, S. Kenney, M.R. Boyd, New colorimetric cytotoxicity assay for anticancer-drug screening, J. Natl. Cancer. Inst., 82 (1990) 1107-1112.

[60] R.H. Shoemaker, The NCI60 human tumour cell line anticancer drug screen, Nat. Rev. Cancer, 6 (2006) 813-823. 